University of Louisville

ThinkIR: The University of Louisville's Institutional Repository

Electronic Theses and Dissertations

$12-2014$

\title{
Aminooxy reagents in nanomedicine and bioanalysis.
}

Stephanie Mattingly

University of Louisville

Follow this and additional works at: https://ir.library.louisville.edu/etd

Part of the Chemistry Commons

\section{Recommended Citation}

Mattingly, Stephanie, "Aminooxy reagents in nanomedicine and bioanalysis." (2014). Electronic Theses and Dissertations. Paper 1751.

https://doi.org/10.18297/etd/1751

This Doctoral Dissertation is brought to you for free and open access by ThinkIR: The University of Louisville's Institutional Repository. It has been accepted for inclusion in Electronic Theses and Dissertations by an authorized administrator of ThinkIR: The University of Louisville's Institutional Repository. This title appears here courtesy of the author, who has retained all other copyrights. For more information, please contact thinkir@louisville.edu. 


\title{
AMINOOXY REAGENTS IN NANOMEDICINE AND BIOANALYSIS
}

\author{
by \\ Stephanie Mattingly \\ A Dissertation \\ Submitted to the Faculty of the \\ College of Arts \& Sciences of the University of Louisville \\ In Partial Fulfillment of the Requirements \\ For the Degree of \\ Doctor of Philosophy \\ Department of Chemistry \\ University of Louisville \\ Louisville, Kentucky
}

December 2014 



\title{
AMINOOXY REAGENTS IN NANOMEDICINE AND BIOANALYSIS
}

\author{
by \\ Stephanie Mattingly \\ A Dissertation Approved on
}

December 2, 2014

by the Following Dissertation Committee:

Dissertation Director: Dr. Michael H. Nantz

Dr. Christopher T. Burns

Dr. Muriel C. Maurer

Dr. Martin G. O'Toole 


\section{ACKNOWLEDGEMENTS}

I am deeply grateful for the mentorship of my research advisor, Dr. Michael Nantz. He is an inspiring teacher and a dedicated researcher who is a master at motivating his students to reach their highest potential. From the start he has been committed to cultivating my academic and professional development, and that constant support has given me an environment in which to thrive. Above all, he has been a great sport for all the hijinks that I had absolutely no part in.

I have had the opportunity to learn from a number of excellent collaborators. I thank Dr. Teresa Fan, Dr. Richard Higashi, and Dr. Geoffrey Clark for teaching me techniques in biochemistry and analytical chemistry. I have also had the privilege of working with some amazing research colleagues. Thank you Souvik, Xuan, Sara, Ali, Ralph, Raju, Mumiye, and Seb for all the help, the laughs, and the memories.

I also want to express my gratitude to my wonderful parents, Amy and Steve, who have supported me in all of my efforts, academic and otherwise. They have taught me that with hard work nothing is out of reach. They are shining examples of integrity and dedication and their love has helped me through the tough times. I also thank my brother and best friend, Bart, who keeps me sane and grounded ... when he isn't putting lifesized cutouts of Star Trek characters in my shower. 


\title{
ABSTRACT \\ AMINOOXY REAGENTS IN NANOMEDICINE AND BIOANALYSIS
}

\author{
Stephanie Mattingly
}

December 2, 2014

The development of nanomaterials as medical diagnostic and therapeutic agents is a rapidly expanding field. A brief search of the literature revealed that roughly half of all articles related to nanoparticle-based drug delivery were published after the onset of this thesis research, over the past 4 years. A few nano-based formulations have entered into clinical use, including liposomal drug formulations, protein-linked drugs, and nanoparticle-based MRI contrast agents. The chemistry that enables conjugation of a functional cargo, whether a drug, a targeting element, or a radionucleotide, to a nanocarrier is thus a critical component for development. Oximation is one of the simplest and most efficient chemical conjugation methods. It entails condensation of an aminooxy group with an aldehyde or ketone carbonyl yielding an oxime ether. The versatility of oximation is highlighted and exploited in this thesis. 
Chapter 1 provides an overview of the application of iron oxide nanoparticles in medicine, and more specifically, to nanoparticles as delivery agents for the anticancer drug doxorubicin.

Chapter 2 describes an oximation methodology for cellular metabolite derivatization to enhance the detection of carbonyl analytes using mass spectrometry (MS). Integration of carbonyl-selective aminooxy functionality with a quaternary ammonium moiety for MS electrospray enhancement and a hydrophobic domain for sample cleanup led us to design reagent QDA (quaternary dodecyl aminooxy) and its $13 C D 3$ isotopologue *QDA. Analysis of QDA/*QDA-treated lung adenocarcinoma A549 cells established a profile of carbonyl metabolites spanning multiple structural classes showing capability for global carbonyl profiling.

In Chapter 4, hydroxylated cationic oxime ether lipids are described for coating core-shell Fe3O4-SiO2 nanoparticles. Formulation of the nanoparticles produced lipid-

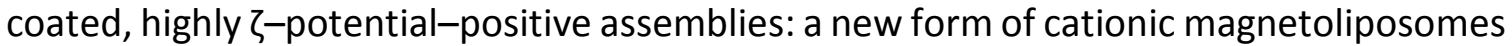
(CML). The CMLs were evaluated as potential drug carriers by integrating into the bilayer a hydrophobically modified analog of doxorubicin. On treatment of MCF-7 breast cancer cells with the drug-loaded CMLs, the assemblies were rapidly internalized and exhibited higher toxicity than treatments with doxorubicin alone. In a separate assessment of the oxime ether lipids, complexation with nucleic acids to form cationic lipoplexes similarly showed high cellular uptake and marked them as viable candidates for siRNA delivery.

Chapter 5 provides all experimental procedures for the aforementioned science. 


\section{LIST OF FIGURES}

Figure 1.1. Representative oximation reaction ....................................................... 2

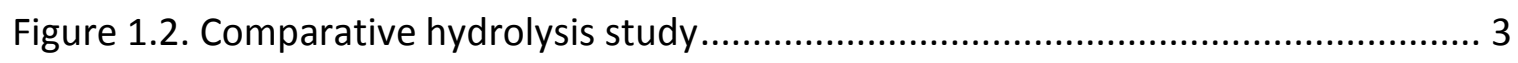

Figure 1.3. Comparison of alpha hydrogen pKa for ketone versus oxime ether .............. 4

Figure 1.4. Representative quaternary aminooxy (QAO) …..................................... 4

Figure 1.5. Agents for the derivatization of carbonyl compounds. .............................. 8

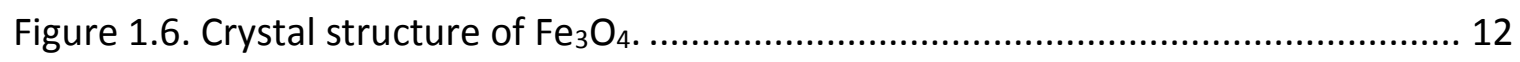

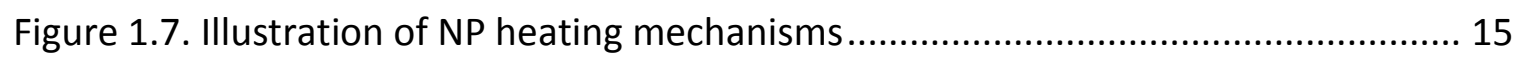

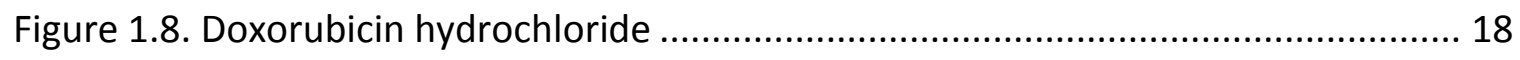

Figure 1.9. Dox covalently linked to superhigh-magnetization nanocarriers................. 19

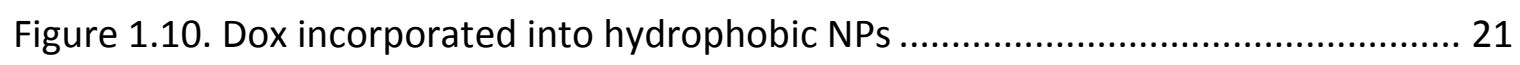

Figure 1.11. Dox in a reducing-polymer/PEG-capped nanoassembly .......................... 21

Figure 1.12. TEM image of NPs on mesoporous silica spheres. ................................... 23

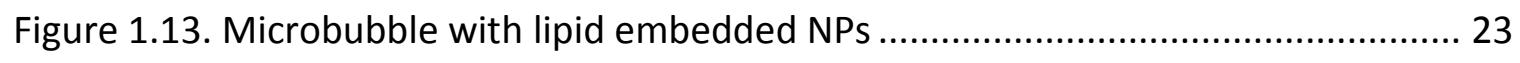

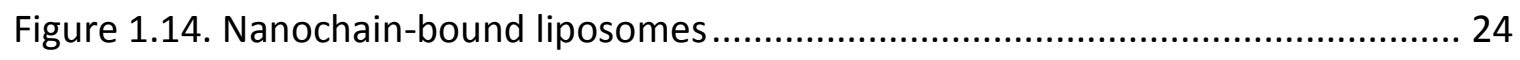

Figure 2.1. Representative structure of derivatizing agent ..................................... 28

Figure 2.2. Stacked $1 \mathrm{H}$ NMR spectra of alcohol 2.3 and $\mathrm{QDA} \cdot \mathrm{Br}$ in $\mathrm{CDCl}_{3} \ldots \ldots \ldots \ldots \ldots \ldots . . . . . . . . . .30$

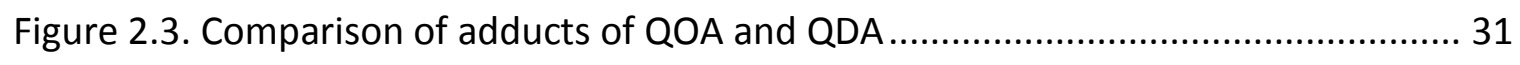

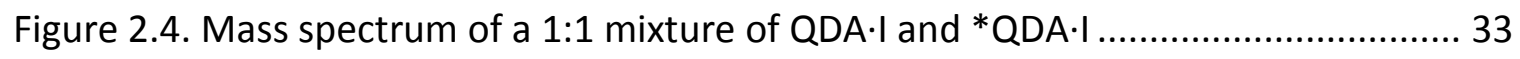


Figure 2.5. QDA bromide and *QDA iodide extraction of standards

Figure 2.6. Derivatization of carbonyl standards and A549 cell extract........................ 37

Figure 2.7. Companion peaks reveal QDA adducts of carbonyl metabolites .................. 40

Figure 2.8. Log-log plot of concentration vs ion count for four *QDA standards ........... 42

Figure 2.9. Uniformly ${ }^{13} \mathrm{C}$-labeled pyruvate spiked into cell extracts .......................... 43

Figure 2.10. Scatter plot of the ion counts of QDA- ${ }^{13} C_{3}$-pyruvate ............................ 44

Figure 3.1. Mössbauer spectrum of NPs prepared by coprecipitation .......................... 51

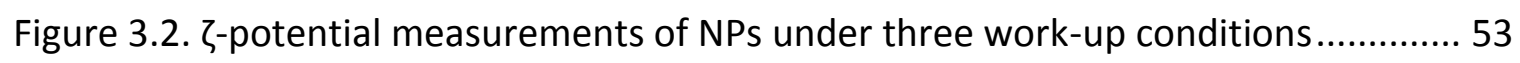

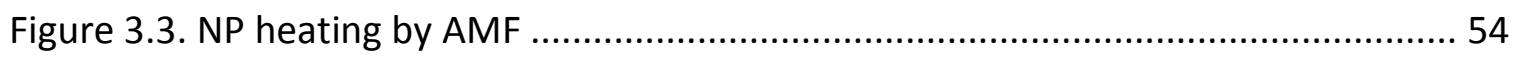

Figure 3.4. Panel of variably hydroxylated ammonium salt ..................................... 55

Figure 3.5. Representative TGA of variably hydroxylated ammonium salts ..................56

Figure 3.6. TGA results of bound panel members .................................................. 57

Figure 3.7 QAOs with variable hydroxyl substituents. ............................................ 59

Figure 3.8. ${ }^{1} \mathrm{H}$ NMR expansion of methylene region of $\mathrm{QAO}-2 \mathrm{OH}$ in $\mathrm{D}_{2} \mathrm{O} \ldots \ldots \ldots \ldots \ldots \ldots \ldots . . . . . . . . . .60$

Figure 3.9. Cationic aminooxy-based oxime ether conjugates of FITC-CHO ................... 61

Figure 3.10. Fluorescence release of three formulations of coated NPs ...................... 61

Figure 3.11. Doxorubicin hydrochloride and oxime ether conjugates ......................... 63

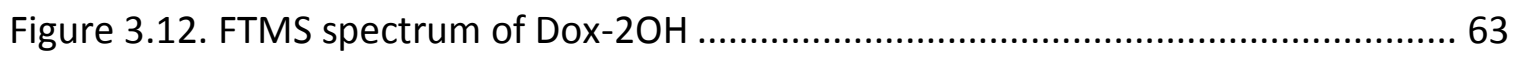

Figure 3.13. UV-Vis detection of Dox analogs released by pulsed AMF........................ 65

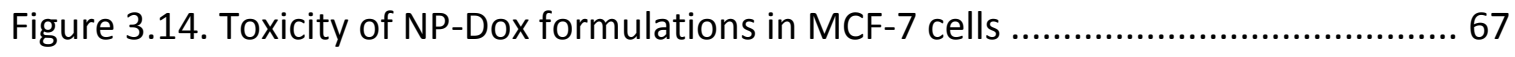

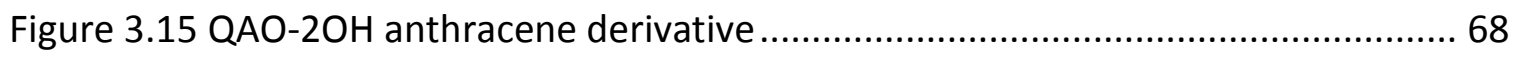

Figure 3.16. AMF-mediated release of anthracene derivative.................................... 69 
Figure 3.17. Traditional xanthate preparation and ferric xanthate variant.

Figure 3.18. Strategies for incorporating organics onto sulfur-modified NPs.

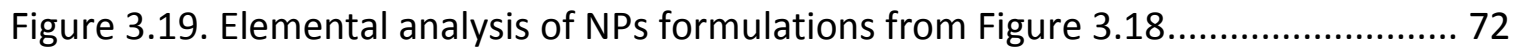

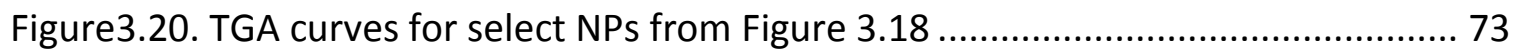

Figure 3.21. TGA of CS2-modified NPs with variably hydroxylated ammonium salts..... 74

Figure 3.22. Proposed mechanism for oxidation of $\mathrm{COS}$ to $\mathrm{CO}_{2}$ on hematite................ 76

Figure 3.23. TGA of $\mathrm{Fe}_{3} \mathrm{~S}_{4}$ NPs or QAO-2OH anthracene-loaded particles. ..................... 77

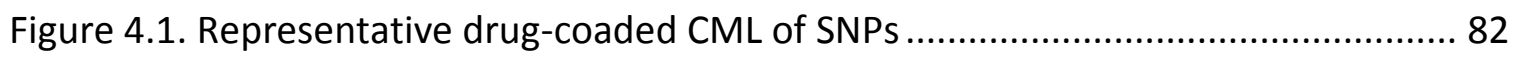

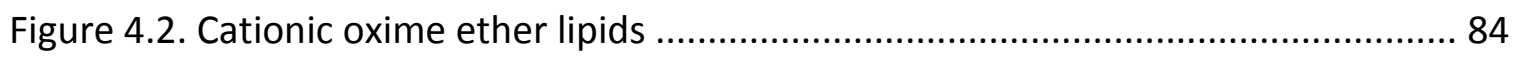

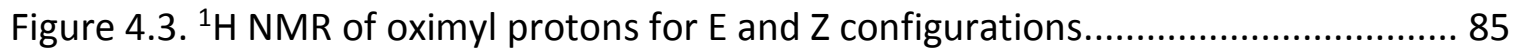

Figure 4.4. Nucleophilic addition of a secondary amine to C-3 of glycidol .................... 86

Figure 4.5. Calculated exact mass of 4.6 and FTMS spectrum of the observed mass...... 86

Figure 4.6. Stacked ${ }^{13} \mathrm{C}$ NMR of Dox and Dox-AH showing hydrazone formation............ 88

Figure 4.7. Dynamic light scattering measurements of CML diameter ........................ 89

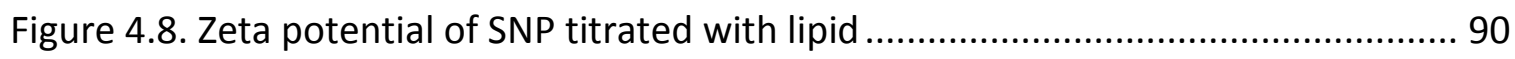

Figure 4.9. Diameter versus percent Dox-AH in a liposomal formulation..................... 91

Figure 4.10. Cytotoxicity of CML treatments in MCF-7 cells ...................................... 93

Figure 4.11. Bright-field and FL images of MCF-7 cells after CML treatment ................ 94

Figure 4.12. First generation oxime ether lipids for gene transfection ........................ 96

Figure 4.13. Binding affinity by fluorescence anisotropy ......................................... 97

Figure 4.14. Transfection efficiency of oxime ether lipids ...................................... 98

Figure 4.15. Viablilty of MDA-MB-231 cells with liposome/RNA complexes .................. 99 
Figure 4.16. Silencing of GFP by lipoplexes with DS RNA ...................................... 100 


\section{LIST OF SCHEMES}

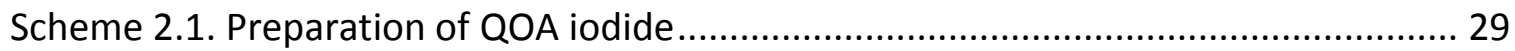

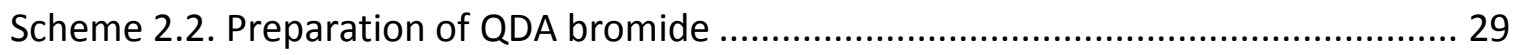

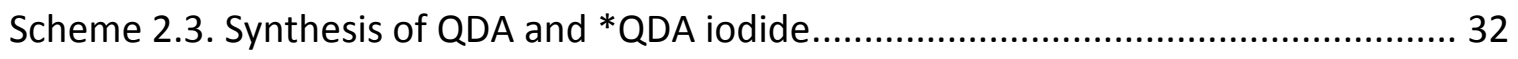

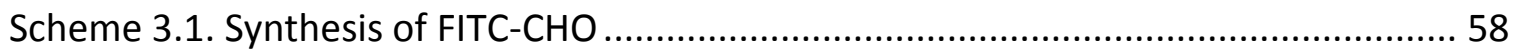

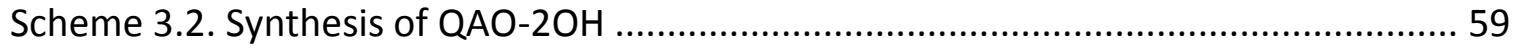

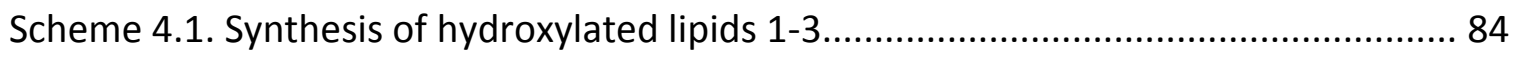

Scheme 4.2: Preparation of Doxorubicin hexadecanyl acyl hydrazone, Dox-AH ............ 87 


\section{TABLE OF CONTENTS}

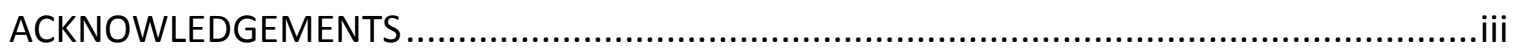

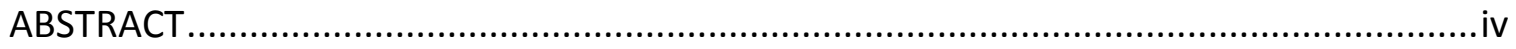

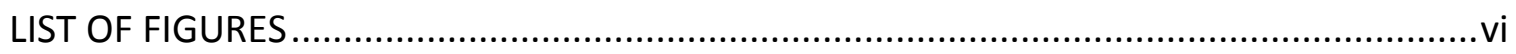

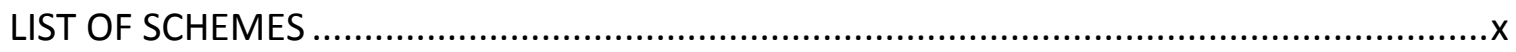

CHAPTER 1 AMINOOXY REAGENTS IN NANOMEDICINE AND BIOANALYSIS ................... 1

1.1. Aminooxy functionality and oximation .......................................................... 2

1.2. Quaternary ammonium salts ....................................................................... 4

1.3. Derivatization reagents for mass spectrometry ................................................ 6

1.4. Iron oxide nanoparticles in nanomedicine ..................................................... 9

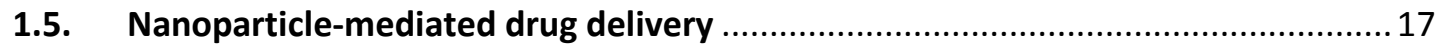

CHAPTER 2 AMINOOXY REAGENTS FOR CHEMOSELECTIVE DERIVATIZATION OF

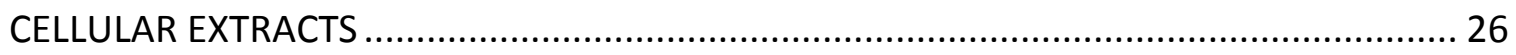

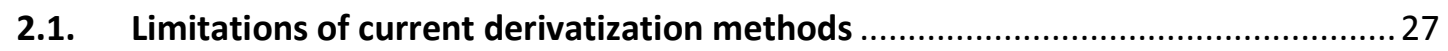

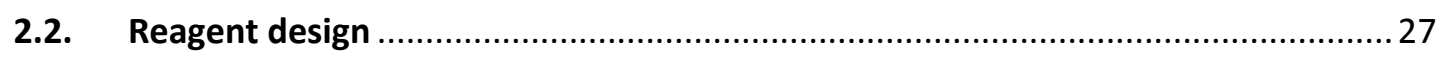

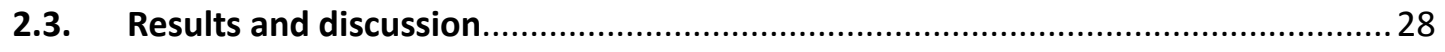

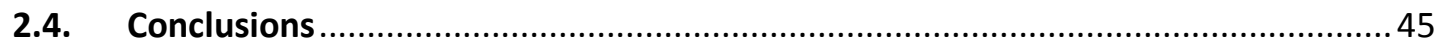

CHAPTER 3 MAGNETIC NANOPARTICLES FOR AMF-MEDIATED DRUG DELIVERY ........... 47

3.1. Externally triggered release of drugs by AMF ................................................. 48

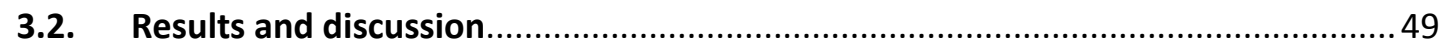

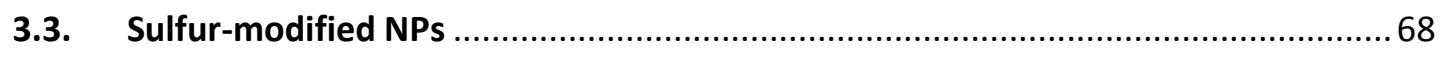

CHAPTER 4 CATIONIC MAGNETOLIPOSOMES FOR DRUG DELIVERY ............................. 78

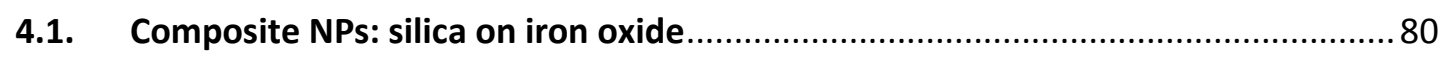

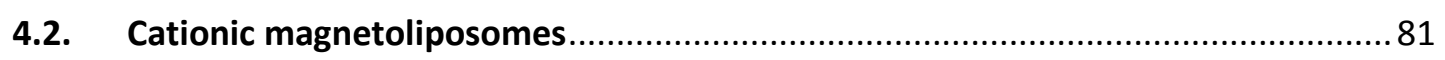

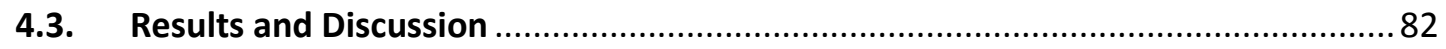




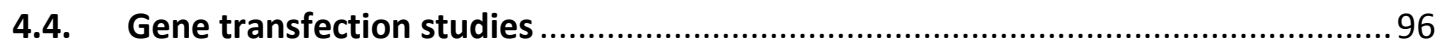

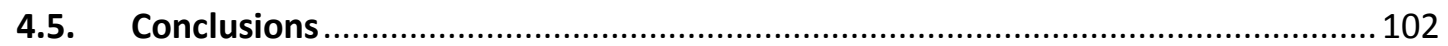

CHAPTER 5 EXPERIMENTAL PROCEDURES .......................................................... 104

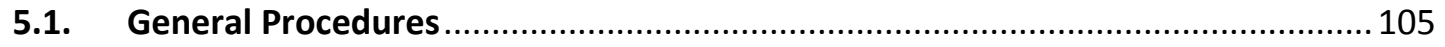

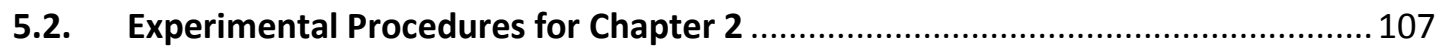

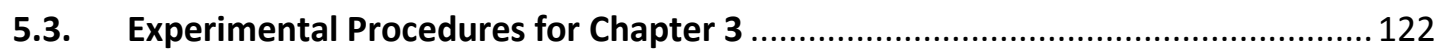

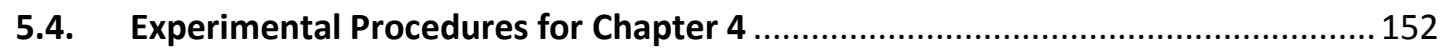

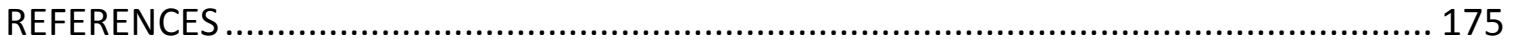

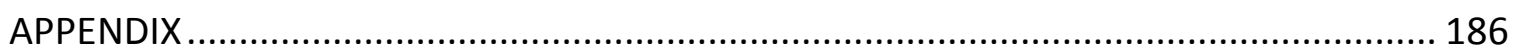

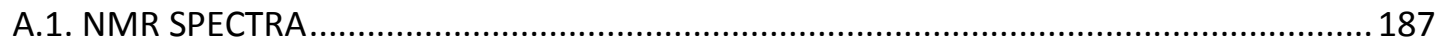

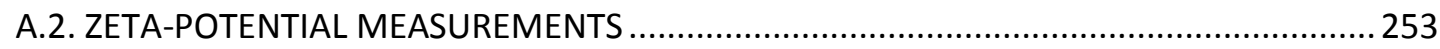

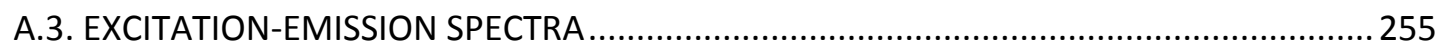

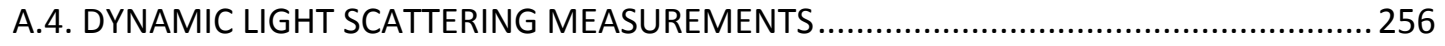

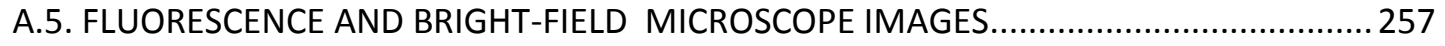

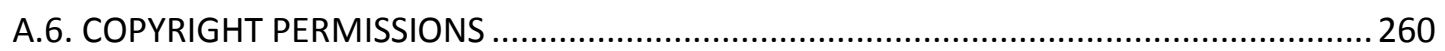

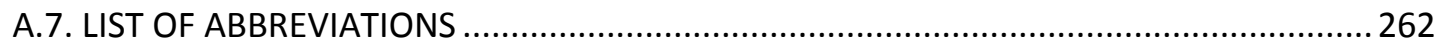

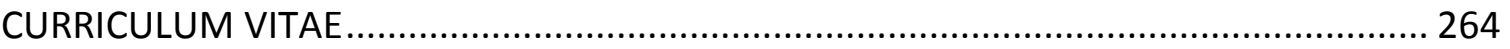




\section{CHAPTER 1 AMINOOXY REAGENTS IN NANOMEDICINE AND BIOANALYSIS}

1.1. Aminooxy functionality and oximation

1.2. Quaternary ammonium salts

1.3. Derivatization reagents for mass spectrometry

1.4. Iron oxide nanoparticles in nanomedicine

1.4.1. Nanoparticle preparation methods

1.4.2. Crystal phase and magnetic properties

1.4.3. Magnetic targeting and imaging

1.4.4. Heating by alternating magnetic field

1.4.5. Surface modifications

1.5. Nanoparticle-mediated drug delivery

1.5.1. Doxorubicin

1.5.2. Progress in nanoparticle-mediated doxorubicin delivery 


\subsection{Aminooxy functionality and oximation}

Oximation, the condensation of an aminooxy moiety with an aldehyde or ketone (Figure 1.1), is one of a select few that meet the criteria for the term "click chemistry" established by K. Barry Sharpless. ${ }^{1}$ Click chemistry reactions have high atom and reaction efficiency, innocuous byproducts, easy purification, and are insensitive to air and moisture. They take their cue from nature, forming covalent links between discrete molecules via carbon-heteroatom bonds. Oximation is a model click reaction in that it usually occurs spontaneously, at room temperature, in numerous solvents, requires no heat, and produces robust chemical bonds with water as its only byproduct. It is an elegant reaction that can link independently functionalized components thus enabling convergent synthesis, and because of its high chemoselectivity, it is suitable for a broad range of tethering reactions. The great versatility of oximation chemistry is reflected in this thesis - my investigations have applied aminooxy reagents to such diverse applications as metabolite derivatization, nanomaterial functionalization, drug delivery, and gene transfer.

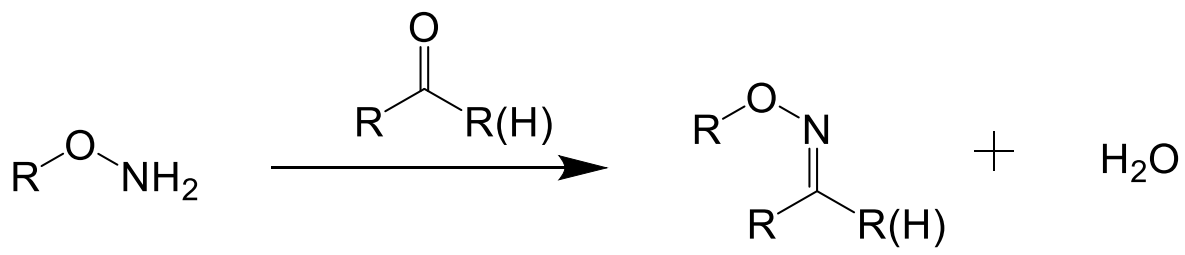

Figure 1.1. Representative oximation reaction: an aminooxy condenses with an aldehyde or ketone to form an oxime ether.

The high stability of the products of oximation, oxime ethers, comes from the unique electronic structure of the functionality. In comparison to analogous functional 
groups, hydrazones and acyl hydrazones, oxime ethers have much slower rates of hydrolysis. Jeet Kalia and Ronald Raines compared the rates of hydrolysis for these linkages and determined, as depicted in Figure 1.2, that oxime ether 1 had hydrolytic rates approximately 600 fold and 300 fold slower than hydrazones 2 and 3 , respectively. This effect is likely due to greater inductive electron donation from oxygen into the pi system as compared to nitrogen. ${ }^{2}$

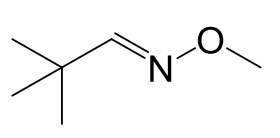

oxime ether 1

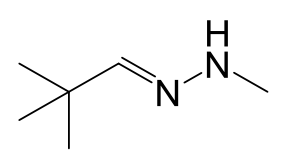

hydrazone 2

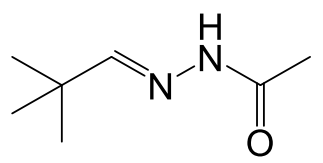

acyl hydrazone 3

Figure 1.2. Compounds examined in a comparative hydrolysis study ${ }^{2}$ of oxime ethers, hydrazones, and acyl hydrazones

Whereas the carbonyl carbon of most functional groups is electropositive, in the case of the $\mathrm{sp}^{2}$ carbon of oxime ethers, electron donation from the attached heteroatoms weakens the polarity of the bond. An indicator of this phenomenon can be found in comparisons of the alpha hydrogen $\mathrm{p} K_{\mathrm{a}}$ 's for oxime ethers versus ketones. The acidity of alpha hydrogens is heavily influenced by the capacity of the adjacent pi system to delocalize electron density through resonance. It is thus an indirect indicator of the electrophilicity of the carbonyl carbon. James Ciula and Andrew Streitwieser have examined the alpha hydrogen $\mathrm{p} K_{\mathrm{a}}$ 's for diphenylacetone and an oxime ether analog (Figure 1.3). ${ }^{3}$ A tenfold higher $\mathrm{p} K_{\mathrm{a}}$ for the oxime ether species was reported; this is evidence of a dramatic reduction in electrophilicity for oxime ether carbonyl carbons. 
Given that hydrolysis proceeds through nucleophilic attack of water on this carbon, the reduction in its electrophilicity can be understood to promote a high degree of stability for the linkage.
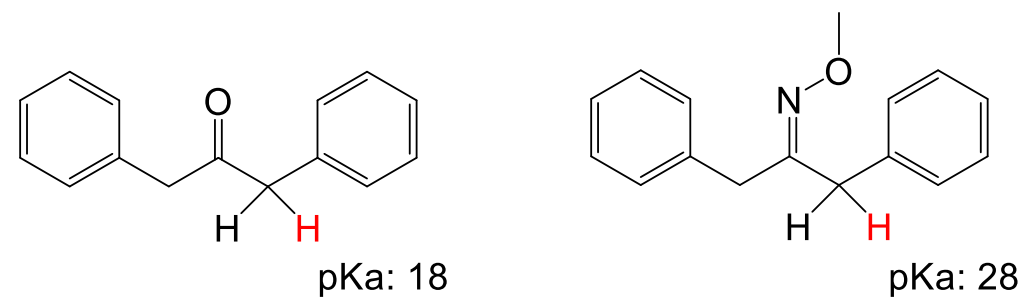

Figure 1.3. Comparison of alpha hydrogen pKa for ketone versus oxime ether

\subsection{Quaternary ammonium salts}

The major focus of this work is the synthesis and application of novel compounds that combine aminooxy functionality with quaternary ammonium moieties. Such quaternary aminooxys (QAOs), exemplified in Figure 1.4, are adapted both for oximation chemistry and the aqueous environment of biological applications.

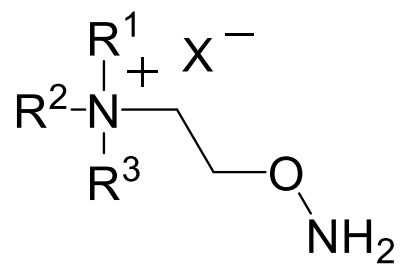

QAO

Figure 1.4. Representative quaternary aminooxy (QAO) 
Quaternary ammonium salts are a useful class of reagents. The four substituents of the quaternary nitrogen provide opportunity for functional variety. Depending upon the hydrophobicity of the attached substituents, amphiphilic species can be prepared with solubility in aqueous and organic solvents. For this reason, such amphiphiles are used commercially as phase transfer catalysts and surfactants. They are also relatively inexpensive, so their use in industry is more common than other phase transfer catalysts, such as phosphonium salts and crown ethers. ${ }^{4}$ Another variant of this type of amphiphile, dual-chain cationic lipids, are often used in gene delivery owing to their ability to condense DNA and transport it across cell membranes; ${ }^{5}$ this activity will be discussed in detail in Chapter 4.

The preparation of ammonium salts is generally straightforward. The protecting group chemistry for nitrogen is well established and the products of quaternization, being salts, can often be isolated by recrystallization, thereby avoiding more costly chromatographic purification.

The custom QAOs we describe in this work were utilized in several different applications. Chapter 2 details the way in which a QAO reagent was used to capture cellular aldehydes and ketones from biological samples, as well as the how the permanent positive charge of the quaternary nitrogen was exploited to maximize the signal intensity of the conjugated metabolites in mass spectral detection. In chapters 3 and 4 , oximation allowed the linking of QAOs to fluorophores and drugs; in this case, the cationic ammonium group facilitated electrostatic attraction to negatively charged iron oxide and silica-coated nanoparticles. Finally, as disclosed at the end of chapter 4, cationic oxime 
ether lipids with quaternary ammonium headgroups were studied for their gene transfer activity. In subsequent sections of this introductory chapter, I give the background for traditional carbonyl derivatization strategies for mass spectrometry (MS), and iron oxide nanoparticle functionalization for drug delivery.

\subsection{Derivatization reagents for mass spectrometry}

The analysis of cellular aldehydes and ketones is of considerable importance in the field of metabolomics since carbonyl species are pervasive intermediates of normal metabolism as well as common products of oxidative stress, ${ }^{6-7}$ which has been implicated in the pathogenesis of many diseases including cancer. ${ }^{8}$ The detection and identification of oxidized cellular species, in general, has become an important focus of research efforts in metabolomics where potential early markers of disease are sought. ${ }^{9-10}$ The identification of polar aldehyde and ketone metabolites from aqueous cell extracts is particularly challenging owing to their low-abundance, high reactivity, and the difficulties associated with their extraction from the cell matrix. Mass spectrometry (MS) has the sensitivity required for observing these low-abundant species, but due to insufficient mass accuracy and resolution, most MS methods are coupled with chromatography to distinguish analytes with similar $m / z$. Fourier-transform ion-cyclotron resonance MS (FTICR-MS), however, does not suffer these limitations and can provide unique molecular formulae of most metabolites. In addition, FT-ICR-MS can achieve simultaneous resolution of ${ }^{13} \mathrm{C}$ isotopologues and distinguish them from other elemental isotopologues

(such as ${ }^{15} \mathrm{~N}$ or ${ }^{2} \mathrm{H}$ ). ${ }^{11}$ However, direct infusion FT-ICR-MS is often compromised by 
unstable sample introduction (e.g., electrospray) and low ion yields due to interference from cellular salts and/or interaction with matrix ions in aqueous biological extracts. Consequently, metabolite derivatization is a strategy that can be employed to overcome these challenges.

In the case of aldehyde and ketone metabolites, derivatization is commonly performed to facilitate liquid chromatography with optical or MS detection (LC-MS) or gas chromatography coupled to mass spectrometry (GC-MS). Such derivatization reagents either have distinct absorbances (for optical detection), preserve or increase volatility (GC), or have easily ionizable groups for MS detection, such as those exemplified in Figure 1.5. ${ }^{6,12-17}$ These reagents react with carbonyls via their amine, ${ }^{12}$ hydrazine, ${ }^{14}$ hydrazide, ${ }^{18-20}$ or aminooxy ${ }^{21-22}$ moieties to form corresponding imine, hydrazone, acyl hydrazone, or oxime ether adducts, respectively. For example, dinitrophenylhydrazine (DNPH) reacts with aldehydes and ketones to form hydrazone derivatives that can be more readily detected due to characteristic UV absorbances. ${ }^{6,14}$ Similarly, $0-2,3,4,5,6-$ (pentafluorobenzyl)hydroxylamine (PFBHA) has been used to form stable, and readily ionizable oxime ether derivatives from aldehydes and ketones. ${ }^{21-22}$ The silylation of $\alpha, \beta-$ unsaturated ketones, using a catalyst to facilitate ketone to enol tautomerization and thus enol silylation, also has been performed to improve the GC-MS analysis of this compound class. ${ }^{23}$ Some of these reagents also employ a permanent positive charge to enhance electrospray MS sensitivity. ${ }^{16-18,20,24-26}$ While still suitable for analysis by LC-MS, this approach precludes the use of GC since the resultant charged adducts have lower volatility. The added charge enhances MS sensitivity and, more importantly, enables 
direct MS analysis under milder, non-ionizing conditions. For example, the hydrazidebased derivatization agent 4-hydrazino-4-oxobutyl-[tris(2,4,6-trimethoxyphenyl)phosphonium bromide (TMPP-PrG) reacts with aldehydes and ketones and contains a quaternary phosphorous to impart a permanent positive charge on its adducts. ${ }^{27}$ Additionally, Girard reagents, ${ }^{17,}{ }^{26}$ such as Girard-P, also contain hydrazide functionality for reaction with carbonyl substrates and a quaternary ammonium moiety to enhance water solubility. Such reagents have been used to enhance detection in secondary ion MS (e.g. Xe ${ }^{+},{ }^{26}$ LC-MS, $^{28}$ and electrospray ionization MS (ESI-MS). ${ }^{29}$ More recently, an aminooxy-based reagent for carbonyl capture was developed by Yuan et al. ${ }^{30}$ in which a biotinylated region of the reagent was used to separate conjugated carbonyls from other cellular species on streptavidin beads. In this case, release of the derivatized metabolites was achieved through activation of a photo-cleavable linker.<smiles>NNc1ccc([N+](=O)[O-])cc1[N+](=O)[O-]</smiles>

DNPH<smiles>NNC(=O)CCCP(Br)(Br)(Br)Br</smiles>

TMPP-PrG<smiles>NOc1c(F)c(F)c(F)c(F)c1F</smiles>

PFBHA<smiles></smiles>

Girard-P reagent

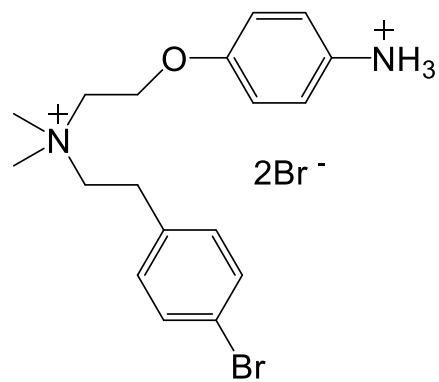

4-APEBA

Figure 1.5. Agents for the derivatization of carbonyl compounds. 
The disadvantages in these reported approaches include multistep procedures for metabolite derivatization, potentially damaging UV light exposure, and/or lengthy chromatographic separation of the resultant metabolite adducts. Chapter 2 will relate our strategy for using direct infusion FT-ICR-MS to analyze crude biological extracts using a QAO reagent. Like several of the reagents described above, the reagent we designed bears a permanent positive charge for ready conversion of carbonyl metabolites into stable, non-volatile, charged adducts. It was also designed for compatibility with aqueous medium. Where it departs from traditional reagents is in the addition of a hydrophobic domain optimized for partitioning of charged adducts into an organic solvent for enhanced electrospray stability. We apply this new cationic amphiphile to the global profiling of carbonyl metabolites directly from crude aqueous extracts of cancer cells.

\subsection{Iron oxide nanoparticles in nanomedicine}

The introduction of nanoparticles into clinical applications is at the cutting edge of medical science. The scale of nanoparticles, typically 10-100 nm, makes them perfectly suited for operating in biological systems. The standard tools of medicine inhabit vastly different size domains; molecular drugs are on the order of a nanometer, and a scalpel, at the other extreme, is roughly 50 million times larger. With the emergence of nanomedicine, there is another tool for medical science, one that is much closer to the micrometer scale of cells. Although the application of nanoparticles in clinical practice has been limited, it is a rapidly expanding field with a promising future. 
Iron oxide, $\mathrm{Fe}_{3} \mathrm{O}_{4} / \mathrm{Fe}_{2} \mathrm{O}_{3}$, nanoparticles (NPs) are a particularly attractive class of nanomaterial. They possess intrinsic magnetic properties that enable both diagnostic and therapeutic procedures, such as magnetic resonance imaging (MRI) through contrast enhancement, ${ }^{31}$ magnetic targeting, ${ }^{32}$ and thermotherapy through inductive heating using an alternating magnetic field. ${ }^{33}$ Moreover, the attachment of targeting ligands ${ }^{34}$ and drugs demonstrates their potential for achieving the ultimate goal of directed, minimal-dose chemotherapy. This versatility makes magnetic nanoparticles appealing candidates as multifunctional drug delivery systems.

\subsubsection{Nanoparticle preparation methods}

Iron oxide nanoparticles of $\mathrm{Fe}_{3} \mathrm{O}_{4}$ (magnetite) or $\gamma-\mathrm{Fe}_{2} \mathrm{O}_{3}$ (maghemite) can be prepared by a variety of methods. ${ }^{35}$ Among the common chemical syntheses are coprecipitation, thermal decomposition and hydrothermal reactions. In coprecipitation, NPs form from an aqueous mixture of iron II and III chloride salts in the presence of base (Equation 1.1). The method was known as far back as 1852 , but was not well studied until the work of René Massart in the 1980 s. ${ }^{36}$ It is the simplest technique as it is a wet chemistry approach that does not require special equipment. However, coprecipitation has a drawback in that the control of particle size and shape is somewhat limited, and NPs made in this way will have a wider size distribution than in more rigorous syntheses. ${ }^{37}$ In thermal decomposition, NPs are formed from the decomposition and oxidation of organometallic precursors, such as iron acetylacetonate, at high temperature in organic solvents and in the presence of surfactants, typically fatty acids. ${ }^{38}$ The NPs generated by this method are hydrophobic and must be modified to confer aqueous solubility. ${ }^{39}$ In 
hydrothermal reactions, NPs are generated using ferric nitrate under aqueous conditions at high temperatures and pressures. ${ }^{40}$ A number of physical preparations of NPs are also known including gas-phase deposition, electron beam lithography and laser pyrolysis. ${ }^{40}$ In perhaps the most inventive methodology, NPs can also be harvested from bacteria; termed the microbial method, $\mathrm{Fe}(\mathrm{III})$-reducing bacteria incubated with a $\beta$-FeOOH precursor synthesize $\mathrm{Fe}_{3} \mathrm{O}_{4} \mathrm{NPs}$ under anaerobic conditions. ${ }^{35}$

$$
\mathrm{Fe}^{2+}+2 \mathrm{Fe}^{3+}+8 \mathrm{OH}^{-} \rightarrow \mathrm{Fe}_{3} \mathrm{O}_{4}+4 \mathrm{H}_{2} \mathrm{O}
$$

Equation 1.1. Precipitation of iron salts to form $\mathrm{Fe}_{3} \mathrm{O}_{4} \mathrm{NPs}$

\subsubsection{Crystal phase and magnetic properties}

The magnetic iron oxides, $\mathrm{Fe}_{3} \mathrm{O}_{4}$ and $y-\mathrm{Fe}_{2} \mathrm{O}_{3}$, both have inverted spinel crystal structure in which the metal ions are located in the tetrahedral and octahedral interstices of a tight packed face-centered-cubic oxygen lattice. In the case of $\mathrm{Fe}_{3} \mathrm{O}_{4}$ (Figure 1.6), ${ }^{41}$ $\mathrm{Fe}^{3+}$ occupies the tetrahedral coordination sites and $\mathrm{Fe}^{2+}$ and $\mathrm{Fe}^{3+}$, in equal parts, occupy the octahedral sites. Below $851 \mathrm{~K}$ magnetite is ferrimagnetic; the different coordination sites have antiparallel alignment of magnetic moments, but electron hopping between the $\mathrm{Fe}^{2+}$ and $\mathrm{Fe}^{3+}$ ions in the octahedral sites produces a net magnetism. ${ }^{39,}{ }^{42} \gamma-\mathrm{Fe}_{2} \mathrm{O}_{3}$ differs from magnetite in that iron is present in only the +3 oxidation state. For maghemite, a somewhat weaker ferrimagnetism (60-80 emu/g compared to 92-96 emu/g for magnetite $)^{43}$ is still present due to an uneven distribution of the metal ions among the coordination sites, i.e. the presence of vacancies. The aligned magnetic 
moments of iron ions in very small NPs combine to create a single large magnetic moment for each individual particle. These small NPs are said to exhibit superparamagnetism ${ }^{44}$ where in the absence of a magnetic field thermal energy causes particle magnetic moments to orient randomly, thus creating a zero net magnetism. In the presence of a field, however, the magnetic moments of the particles align with the external field. This lack of remnant magnetism allows NPs to amass when a magnetic field is present, but redisperse into colloidal form when released from the field, making them ideal for biological applications. ${ }^{39}$

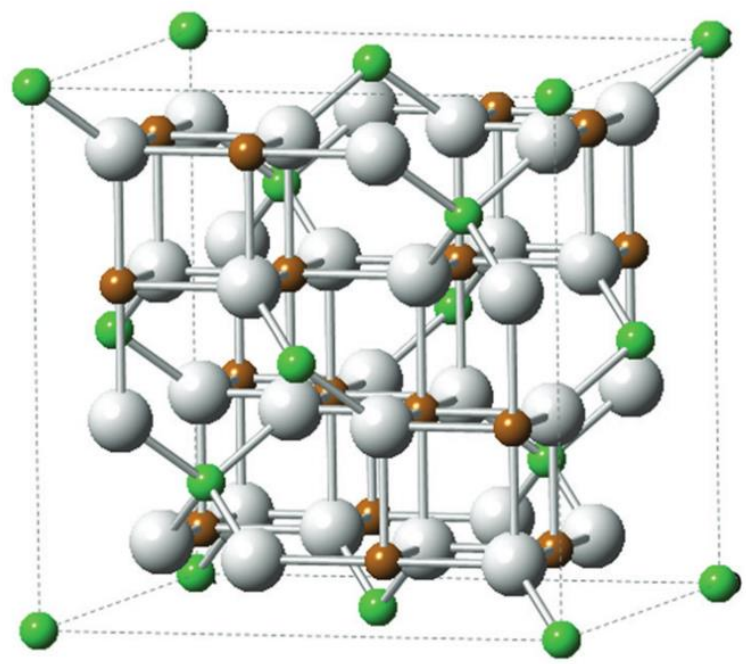

Figure 1.6. Crystal structure of $\mathrm{Fe}_{3} \mathrm{O}_{4}$ : green balls $\left(\mathrm{Fe}^{2+}\right)$, brown balls $\left(\mathrm{Fe}^{3+}\right)$, white balls (O). Reprinted from Chem. Commun. (Cambridge, U. K.) 2011, $47,5130-5141$ with permission from the Royal Society of Chemistry. 


\subsubsection{Magnetic targeting and imaging}

The magnetic properties of NPs enable magnetic targeting through external magnetic field application. In a survey of the literature, several in vivo targeting studies are reported. ${ }^{32,45-46}$ Ma and coworkers, for example, injected alginate-coated NPs into a rat femoral artery and were able to demonstrate retention of the NPs at a target site by magnetic field application. ${ }^{47}$ Similarly, Pisciotti et al. showed accumulation of intravenously injected polyethylene glycol (PEG)-coated NPs in a mouse tumor where an external field was applied. ${ }^{45}$ Although promising, magnetic targeting to this point has been applied only to superficial tumors; deep tissue targeting poses a challenge because the strength of a magnetic field rapidly weakens $\left(r^{-3}\right)$ with distance from the source magnet.

NPs have also been applied in magnetic resonance imaging (MRI) where they provide negative contrast enhancement in NP-bearing tissues. The proton signal intensity in MRI in tissue is influenced by proximity to NPs. Both $T_{1}$ and $T_{2}$ relaxation processes are affected; however, because the NPs produce local field inhomogeneity, faster $T_{2}$ relaxation (decoherence of the transverse nuclear spins) dominates. Thus NPs are used in $T_{2}$-weighted pulse sequences. ${ }^{39}$ There are a few ferrofluids approved by the FDA for MRI use in humans including ferumoxsil and ferrumoxide, siloxane-coated and dextrancoated NPs, respectively. The biocompatibility of such formulations have been investigated. Radiotracer evidence suggests that clinically administered NPs are biodegraded and incorporated into erythrocyte heme iron ${ }^{48}$ or bound to transferrin and 
eliminated through defecation. ${ }^{35}$ Although organic coatings, particularly polymers, can carry their own intrinsic toxicity, ferrofluids are generally regarded as biocompatible. ${ }^{35}$

There is presently a thrust in nanomedicine toward theranostics, the practice of combining therapy and diagnostics. Along those lines, MRI contrast enhancement from NPs could be combined with other therapies enabled by the ferrofluid. One such therapy that has the potential to excel in this capacity is thermotherapy, as described below.

\subsubsection{Heating by alternating magnetic field}

In the presence of an alternating magnetic field, the magnetic properties of NPs cause heat to be generated in a process called induction heating. This can take place through a few different mechanisms depending upon the size of the particles as illustrated in Figure 1.7. ${ }^{49}$ In the case of small superparamagnetic NPs ( $<20 \mathrm{~nm}$ diameter), Néel relaxation is the primary mechanism of heating. In this process the magnetic moment of a NP will reorient to align with the shifting external field direction, and resistance to this change in dipole orientation generates heat. For larger particles (>20 $\mathrm{nm}$ diameter), Brownian motion relaxation is the primary heating mechanism; the particle itself rotates to align its magnetic dipole with the external field, and the friction between the NP surface and the surrounding medium induces heating. The third mechanism of heating, hysteresis losses, similarly occurs in larger particles. Here, the particles are not superparamagnetic but instead are multi-domain particles where regions of the NPs have net magnetic moments oriented randomly with respect to one another. Hysteresis 
heating is a consequence of the growth of the domain best aligned with the magnetic field at the expense of neighboring domains.
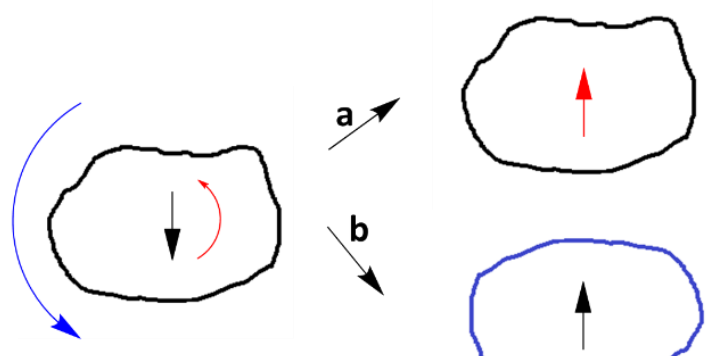

b
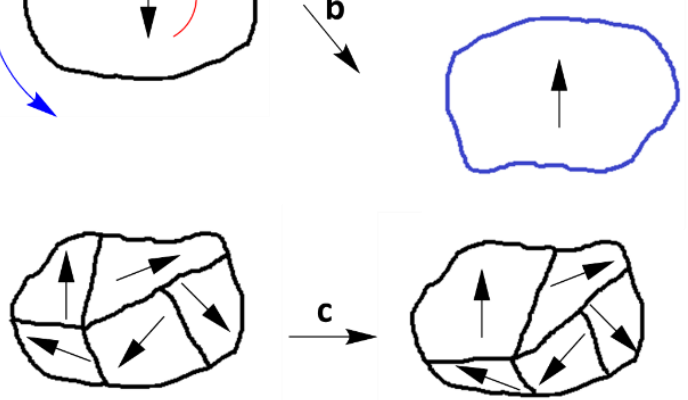

Figure 1.7. Illustration of NP heating mechanisms: (a) Néel relaxation; (b) Brownian motion relaxation; (c) Hysteresis losses

Although this discussion has focused on the mechanisms of NP heating, it is important to note that the increase in temperature of the bulk solution of a ferrofluid is not necessarily indicative of the local environment at the NP surface. There is considerable evidence to suggest that the energy transfer from AMF-induced NPs to attached ligands/polymers has exaggerated effects at the particle interface. ${ }^{50}$ Riedinger et al., for example, used a thermally sensitive azo molecule to probe NP surface temperature at subnanometer distances and found that a local temperature increase up to $44{ }^{\circ} \mathrm{C}$ during one hour of AMF exposure $(17 \mathrm{mT}, 334.5 \mathrm{kHz})$ could be found within 0.5 $\mathrm{nm}$ of the NP surface. ${ }^{51}$ 
The heat-generating ability of NPs exposed to AMF is used clinically in magnetic fluid hyperthermia where ferrofluids are injected into tumor sites and heated by AMF induction. The tumor tissue is heated to between 40 and $45^{\circ} \mathrm{C}$ to achieve hyperthermia, as heating above $45^{\circ} \mathrm{C}$, termed thermoablation, causes tissue necrosis. ${ }^{52}$ Surrounding tissues, absent NPs, are unharmed by a well-directed oscillating field. Hyperthermic temperatures can contribute directly to tumor regression, but they also boost the efficacy of traditional therapies (chemotherapy, radiation) through a process of tumor sensitization. ${ }^{53}$ The established use of hyperthermia therapy has led to the development of instrumentation designed for AMF administration in humans. MagForce Nanotechnologies (AG, Berlin), for example, manufactures units for AMF application to human patients. AMF is also used in NP applications as an external trigger for the release of bound substrates (drugs/fluorophores), as will be discussed shortly.

\subsubsection{Surface modifications}

Surface coating of NPs is typically employed to improve their aqueous solubility, prevent aggregation, and neutralize surface reactivity. There are several classes of coating agents; iron chelators are common, including catechols $s^{43,54}$ and carboxylates, both single molecule (oleic acid, ${ }^{55}$ citrate $^{56}$ ) and polymeric species (alginate, ${ }^{32}$ carboxymethyl dextran ${ }^{57}$ ). These species bind to the iron at the surface of the NP. Hydrogen bond donors for NP surface oxyanions/hydroxyls are frequently used as well, including polymeric amines (polyethylenimine ${ }^{58}$ ), alcohols (polysaccharides, ${ }^{59}$ polyvinyl alcohol $^{58}$ ), and ethers (polyethylene glycol). ${ }^{60}$ Creating composite core-shell 
nanostructures with inorganic material surrounding iron oxide cores is also frequently employed, as these shells can contribute additional functionality to the NPs. Gold-coated NPs, ${ }^{61}$ for example, can be imaged by surface plasmon resonance. Silica coating of iron oxide is also common and will be discussed in detail in Chapter 4.

\subsection{Nanoparticle-mediated drug delivery}

To date, the clinical use of NPs has largely been confined to imaging and hyperthermia therapy, but the greater potential for NPs in nanomedicine is in multimodal therapies that augment the techniques enabled by NP magnetism with attachment of functional molecules, like pharmaceutical agents or targeting ligands. Perfecting the technology of functionalized NPs carries big implications for human health. The following

excerpt ${ }^{62}$ from Amy Pope-Harman concerns progress in the field of cancer treatment and sums up the challenges and the motivations for nanotechnology in medicine quite poignantly.

Clinical medicine has seen tremendous improvements in the past several decades. Despite the progress ... patients still are called on to endure toxic and uncomfortable whole-body chemotherapy and other treatments with the hope that their cancers will succumb. There is expectation, however, that all of this will change radically with the use of nanotechnology . . . allowing improvements in mortality and prevention of suffering from cancer and other devastating diseases . . By working on a nanometer, molecular scale, there is the potential to simultaneously assess and intervene, with accuracy in space and time, which is an ability that has never before been available. 
In subsequent sections of this chapter, I will outline some of the recent innovations in adapting NPs to function as drug carriers. Specifically, this overview will focus on the NP-mediated delivery of the anticancer agent doxorubicin.

\subsubsection{Doxorubicin}

Doxorubicin (Dox, Figure 1.8), is an anthracycline antibiotic known for its potent activity against a broad spectrum of cancers. ${ }^{63}$ Several modes of action for Dox have been detailed in the literature including DNA intercalation, helicase inhibition, free radical generation, and topoisomerase II interaction leading to apoptosis. ${ }^{64}$ In the case of MCF7, the breast cancer cell line used in the in vitro studies described in Chapters 3 and 4, the antiproliferative action of Dox may be related to interference with DNA unwinding. ${ }^{65}$ In the absence of a NP carrier, targeting agent, or liposomal formulation, Dox has nonspecific toxicity and is known to damage kidney tissue and bone marrow, but its most troubling side-effect is cardiotoxicity. ${ }^{66-68}$<smiles>COc1cccc2c1C(=O)c1c(O)c3c(c(O)c1C2=O)C[C@@](O)(C(=O)CO)C[C@@H]3O[C@H]1C[C@H]([NH3+])[C@@H](O)[C@H](C)O1</smiles>

Figure 1.8. Doxorubicin hydrochloride 


\subsubsection{Progress in nanoparticle-mediated doxorubicin delivery}

Dox is frequently the model drug of choice for demonstrating targeted delivery using NP platforms. Such platforms have incorporated Dox in a variety of ways using different methods of attachment. Covalent attachment is a strategy that is occasionally used offering the benefit of good retention of Dox on the particle. The drawback of covalent linkage is that release of the drug often requires cleavage of covalent bonds in the absence of an activator. For example, Hua and coworkers formed an amide linkage between Dox and an activated carboxylic acid at the surface of acidic polymer-coated NPs to demonstrate MRI contrast enhancement and magnetic retention in a mouse hypodermic tumor (Figure 1.9). ${ }^{69}$ Similarly, Li et al. ${ }^{70}$ used silica-coated iron oxide NPs to covalently bind Dox via a urea linkage that tethered the Dox amine to an isocyanate group on the silane monomer used for constructing the silica shell.

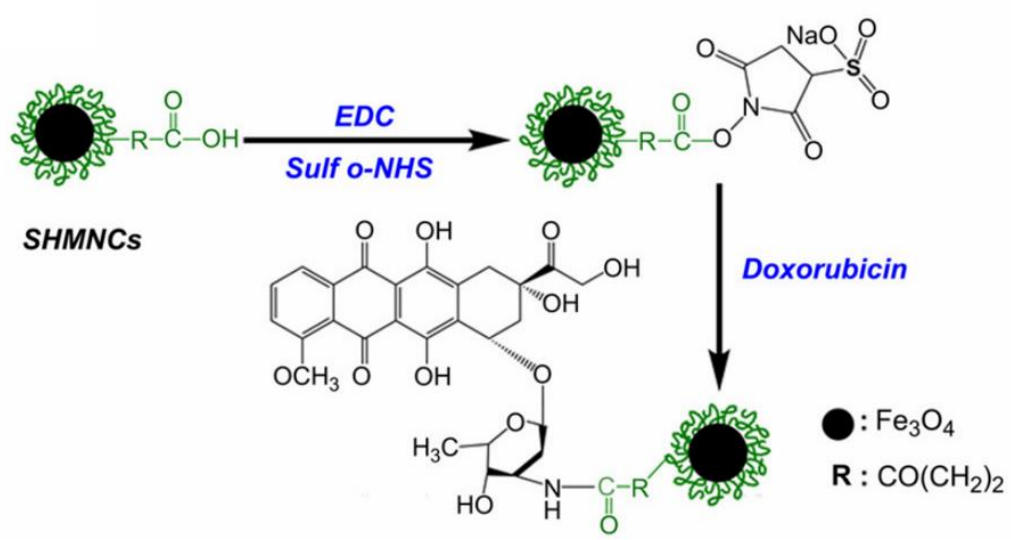

Figure 1.9. Dox covalently linked to superhigh-magnetization nanocarriers (SHMNCs). Adapted from Biomaterials 2011, 32 (34), 8999-9010 with permission from Elsevier. 
Tumor sites are known to be more acidic than healthy tissue, ${ }^{71}$ and for this reason $\mathrm{pH}$-triggered release is sometimes planned into the design for NPs with covalently bound Dox. In this vein, a few investigators have covalently attached Dox to NPs via acidcleavable hydrazone bonds formed from hydrazine/hydrazide-functionalized NPs reacting with the Dox C-13 carbonyl. ${ }^{72-74}$ Patra and coinvestigators, for example, used hydrazide functionalized poly(styrene)-b-poly(acrylic acid) block copolymer to bind Dox on folate-conjugated NPs for targeted delivery to breast and colon cancer cells. ${ }^{73}$ In an alternate approach, that likewise used a pH-based cleavage strategy, Gautier and coauthors installed an $\mathrm{Fe}^{2+}$ ion bridging the oxygens of the Dox quinone system in order to bind Dox directly to NPs. ${ }^{75}$ PEG chains were added to stabilize the linkage and release profiles showed a pH dependence, as protons gradually displaced $\mathrm{Fe}^{2+}$ and freed the Dox from the NP surface. This formulation was found to be effective against MCF-7 cells.

Hydrophobic interactions have also been used as a means to reversibly attach Dox to NPs. Jain et al. ${ }^{55}$ incorporated free base Dox, prepared by bubbling gaseous ammonia into an organic suspension of Dox $\mathrm{HCl}^{76}$ into the hydrophobic shell of oleic acid coated iron oxide NPs. A capping polymer, Pluronic (similar to PEG), was then added to retain the Dox and confer aqueous solubility to the complex. Drug release was achieved slowly over several hours by passive diffusion (Figure 1.10). Chen and coworkers similarly utilized hydrophobic interactions to entrap Dox into the hydrophobic interior of a NP-containing micelle. ${ }^{77}$ Figure 1.11 illustrates this novel formulation in which a reduction sensitive copolymer generates the layered nanostructure pictured. Release of Dox was designed to be mediated by the reducing environment of circulation and cytoplasm. In vitro real- 
time fluorescent monitoring and in vivo tumor growth studies were used to demonstrate the efficacy of the formulation.
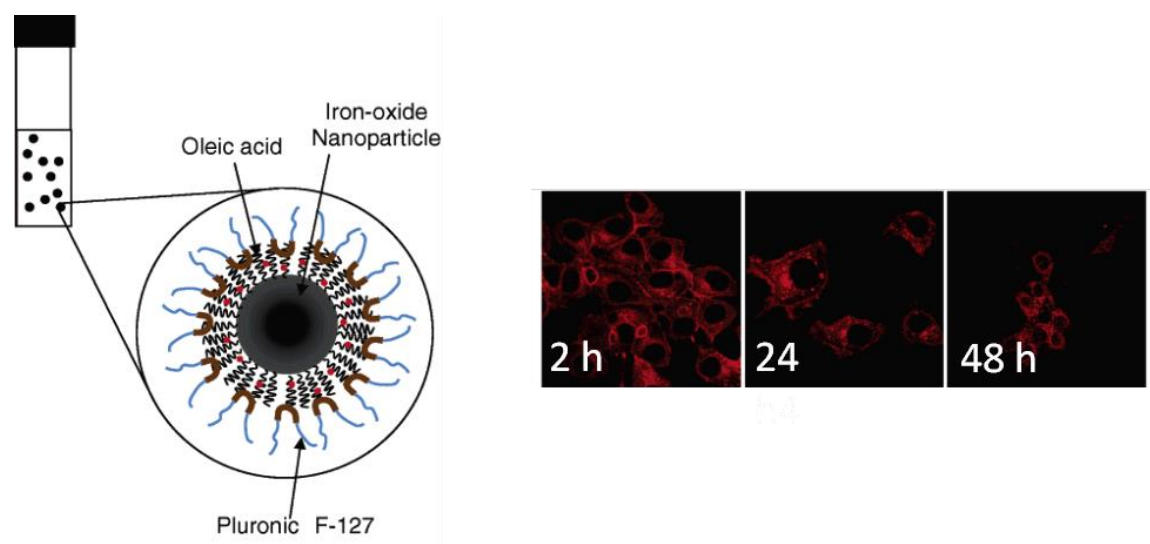

Figure 1.10. Left: charge neutral Dox (red dots) incorporated into an oleic acid hydrophobic layer surrounding a NP; right: confocal laser scanning microscopic images of MCF-7 cells incubated for 2, 24, and $48 \mathrm{~h}$ with the Dox-NPs. Adapted from Molecular pharmaceutics 2005, 2 (3), 194-205 with permission from American Chemical Society. Copyright (2005) American Chemical Society.
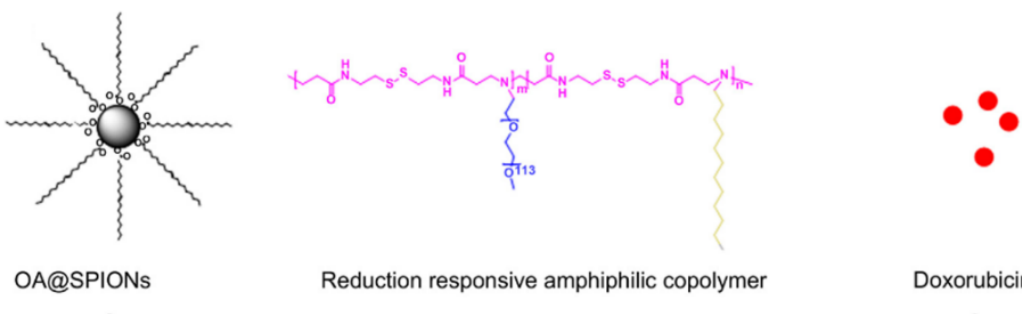

Reduction responsive amphiphilic copolymer

Doxorubicin

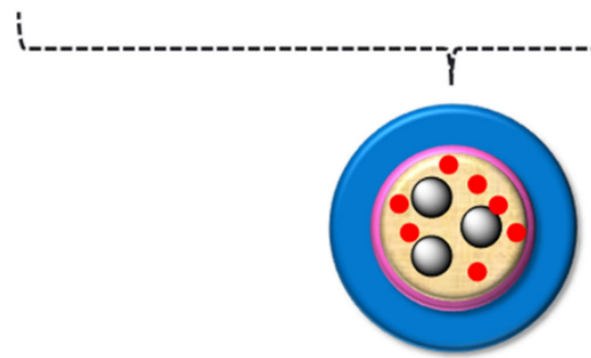

Figure 1.11. Hydrophobic oleic acid nanoparticles coincorporated with Dox into a reducing-polymer/PEG-capped nanoassembly. Adapted from Biomaterials 2014, 35 (4), 1240-1248 with from permission from Elsevier. 
Noncovalent interactions such as electrostatic interactions and hydrogen bonding are likewise frequently used for Dox conjugation to NPs. For example, Lübbe and coworkers in a phase I clinical trial, exploited noncovalent interactions by chemisorbing a Dox analog, epirubicin, to anhydroglucose polymer coated NPs. ${ }^{78}$ The magnetic targeting ability of the drug-loaded NPs were tested in vivo in 14 patients with solid tumors, and targeting was found to be successful in half of the patients. In another noncovalent approach from Lee and coauthors, ${ }^{79}$ mesoporous silica particles were loaded with Dox, then magnetite nanocrystals and PEG chains were attached at the silica surface (Figure 1.12). Dox was administered in this formulation to a mouse tumor model where accumulation of the particles in tumor tissue as well as cell death were observed. Li et al. ${ }^{80}$ used amphiphilic gelatin to incorporate NPs and to electrostatically bind Dox to the carboxylated surface of the gelatin. Calcium phosphate was then used as a capping agent to facilitate acidic $\mathrm{pH}$-mediated degradation of the complex. An in vitro study with HeLa cells verified the release of Dox by confocal laser scanning microscopy. Finally, Fan and colleagues used a lipid to electrostatically bind Dox in a novel construct illustrated in Figure 1.13. ${ }^{81}$ Here, gas bubbles of perfluoropropane were prepared in a lipid sphere that contained embedded NPs in addition to the adsorbed Dox. Next, focused ultrasound was used to rupture the bubbles and simultaneously generating blood-brain barrier openings in an in vivo study with a rat glioma model. The microbubbles were targeted with an external magnet and tracked by MRI. 


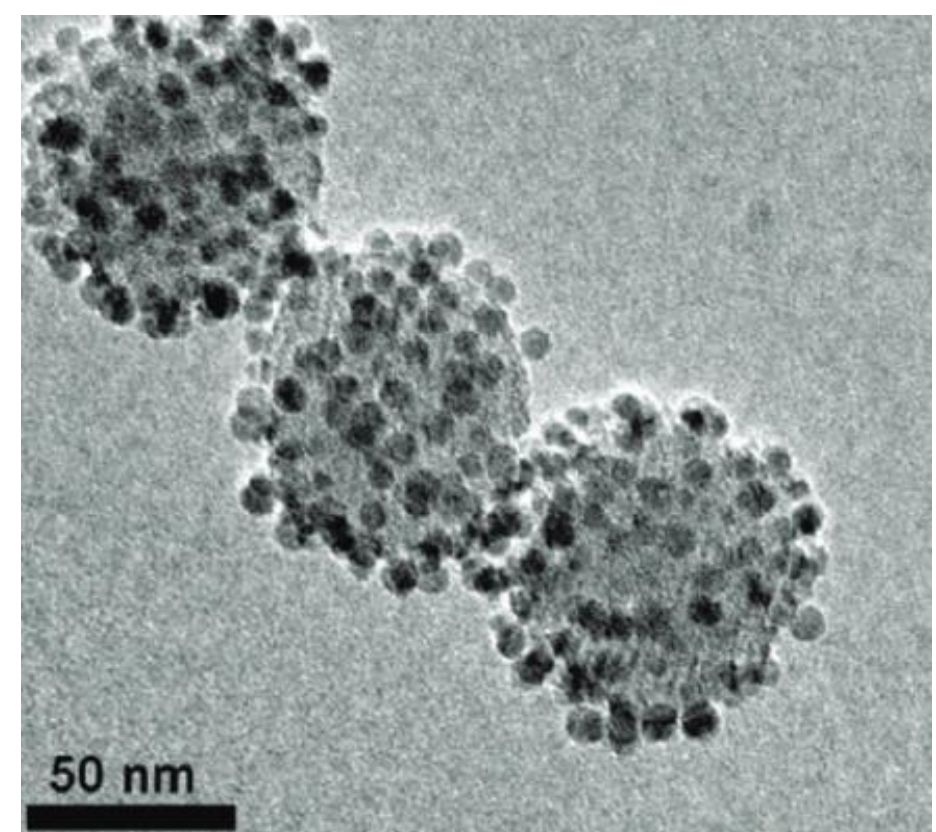

Figure 1.12. TEM image of NP loaded onto the surface of mesoporous silica spheres. Adapted with permission from J. Am. Chem. Soc. 2010, 132 (2), 552-557. Copyright (2010) American Chemical Society.
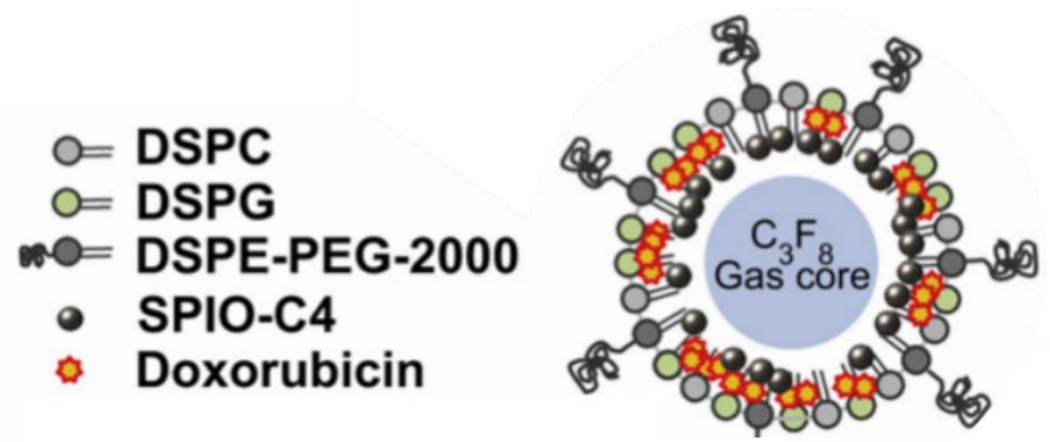

Figure 1.13. Microbubble with lipid embedded NPs (labeled SPIO-C4) Adapted from Biomaterials 2013, 34 (14), 3706-3715 with permission from Elsevier. 
AMF application has also been applied to NPs to effect Dox release, most often in the context of heat-sensitive polymers or gels. For example, Brulé et al. ${ }^{82}$ incorporated NPs and Dox into alginate gel beads and used AMF to induce Dox diffusion from the heated gel. They observed no leakage of Dox at $4{ }^{\circ} \mathrm{C}$, moderate release at $37^{\circ} \mathrm{C}$, and significant release from AMF heating at $53^{\circ} \mathrm{C}$. Peiris and coworkers used the mechanical effect of AMF application, rather than heat generation, to trigger release of Dox from liposomes. ${ }^{83}$ In this creative approach, a string of NPs were tethered by crosslinking chains to a liposome containing encapsulated Dox (Figure 1.14). The authors were able to demonstrate superior tumor distribution of Dox in an in vivo model by the AMFactivated nanochains as compared to Dox liposomes alone.

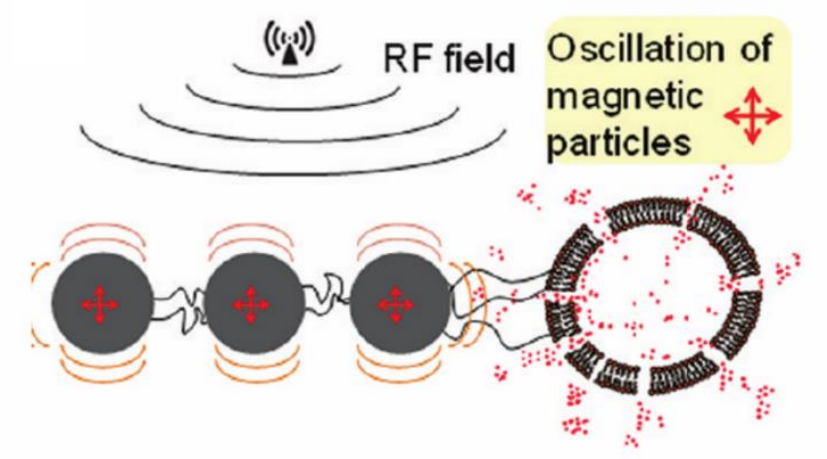

Figure 1.14. Depiction of AMF-triggered release of Dox from nanochainbound liposome. Adapted with permission from ACS Nano 2012, 6 (5), 4157-4168. Copyright (2012) American Chemical Society.

The above reports reflect the most recent advances in nanocarrier-mediated delivery of doxorubicin. Several of these innovations were reported after the onset of 
our own investigations and informed the evolution of our strategies. In Chapters 3 and 4 of this thesis, I will present our contributions to this emergent field of nanotherapy. 


\section{CHAPTER 2 AMINOOXY REAGENTS FOR CHEMOSELECTIVE DERIVATIZATION OF CELLULAR EXTRACTS}

2.1. Limitations of current derivatization methods

2.2. Reagent design

2.3. Results and discussion

2.3.1. Chain length variations

2.3.2. Extraction solvent determination

2.3.3. Stable isotope labeled analog

2.3.4. Counterion effects

2.3.5. Cell extract derivatization and analysis

2.3.6. Metabolite identification

2.3.7. Quantification of pyruvate

2.4. Conclusions 


\subsection{Limitations of current derivatization methods}

Chapter 1 introduced derivatization as a technique for improving the detection by mass spectrometry (MS) of volatile, charge-neutral, or low molecular weight metabolites. The shortcomings of traditional ketone/aldehyde derivatization reagents highlighted earlier included the requirements of harsh reaction conditions (acidic $\mathrm{pH}$ and/or high temperature), and chromatographic separation of derivatives prior to MS detection. We, along with our collaborators, Dr. Teresa Fan, Dr. Richard Higashi, and Mr. Tao Xu, set out to design a derivatization reagent and accompanying methodology that would overcome the drawbacks of the more common approaches to enable an aqueous phase, low-temp conjugation to carbonyl metabolites. We aimed for a minimal processing method suitable for analysis of crude biological extracts using direct infusion Fourier-transform ioncyclotron resonance mass spectrometry (FT-ICR-MS).

\subsection{Reagent design}

There were four key components of our reagent design. We saw in the mild and aqueous-friendly reaction conditions of oximation chemistry an opportunity for facile derivatization of carbonyl metabolites in cell extracts. The formation of oxime ether bonds from an aminooxy reagent to a bioanalyte carbonyl would stabilize the resultant adduct (recall from Chapter 1 the comparison of $\alpha$-proton acidity for 1,3 diphenyl acetone, $\mathrm{pKa} \approx 18$, versus its corresponding methyl oxime ether, $\mathrm{pKa} \approx 28) .{ }^{3}$ The second critical feature of the reagent was the incorporation of a permanent positive charge to maximize MS sensitivity, increase the aqueous solubility of the probe, and generate 
nonvolatile adducts. For this purpose, a quaternary ammonium cation seemed ideal. Third, to overcome the interference of cellular salts and cell matrix ions in the establishment of a stable electrospray, we sought a reagent that, when conjugated to an analyte, could be extracted into an organic solvent. Lastly, we desired a reagent that could easily be labeled with a stable isotope both for the purpose of verification of adducts as carbonyl derivatives, as well as for quantitative MS analysis.

Figure 2.1 is a representative structure of our initial design. Present are an aminooxy moiety for chemoselective carbonyl capture, a cationic ammonium headgroup for MS sensitivity, an aliphatic tail to facilitate organic phase partition, and methyl groups for stable-isotope labeling through quaternization with commercially available and inexpensive iodomethane.

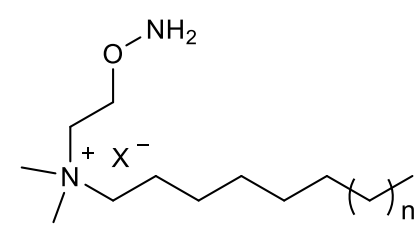

Figure 2.1. Representative structure of derivatizing agent

\subsection{Results and discussion}

\subsubsection{Chain length variations}

To examine the effect of aliphatic chain length on the efficiency of metabolite extraction, dodecyl and octyl versions of the reagent were prepared as depicted in Schemes 2.1 and 2.2 with designations QOA (quaternary octyl aminooxy) and QDA (quaternary dodecyl aminooxy). These preliminary syntheses were three step reactions beginning with quaternization of a tertiary amine with an alkyl halide of the desired chain 
length. A modified Mitsunobu reaction ${ }^{84}$ generated the phthalimide-capped alcohol, and, in the final step, hydrazinolysis of the phthalimide revealed the aminooxy functionality.

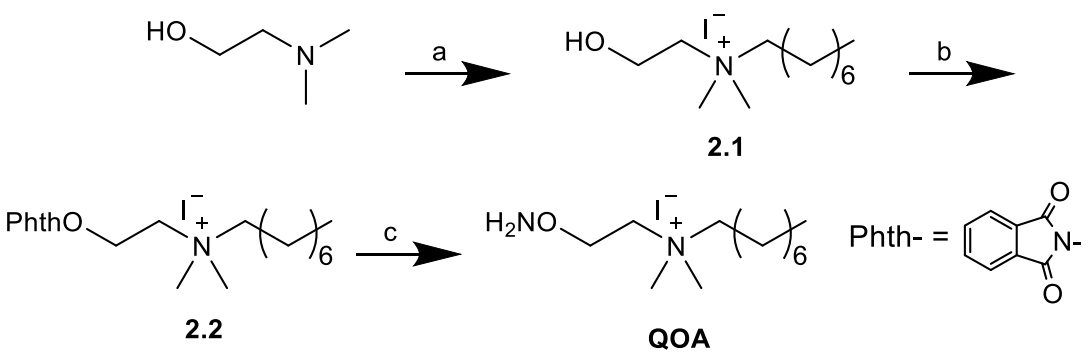

Scheme 2.1. Preparation of QOA iodide. Reagents and conditions: (a) 1iodooctane (1.1 eq), acetone, reflux, $15 \mathrm{~h}, 92 \%$ (b) $\mathrm{N}$-hydroxyphthalimide (1.1 eq), $\mathrm{PPh}_{3}$ (1.1 eq), DIAD (1.1 eq), THF, $0^{\circ} \mathrm{C}-\mathrm{rt}, 12 \mathrm{~h}, 87 \%$ (c) $\mathrm{N}_{2} \mathrm{H}_{4} \cdot \mathrm{H}_{2} \mathrm{O}$ (5 eq), $\mathrm{CH}_{2} \mathrm{Cl}_{2}, \mathrm{rt}, 2 \mathrm{~h}, 92 \%$

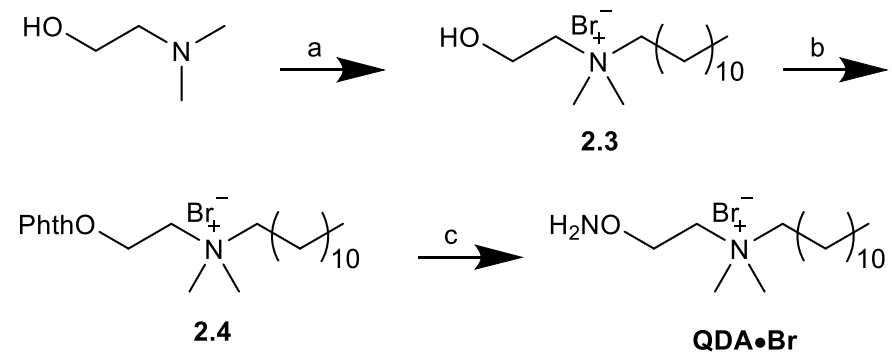

Scheme 2.2. Preparation of QDA bromide. Reagents and conditions: (a) 1bromododecane (1.1 eq), acetone, reflux, 10h, $49 \%$ (b) $\mathrm{N}$ hydroxyphthalimide (1.1 eq), $\mathrm{PPh}_{3}$ (1.1 eq), DIAD (1.1 eq), THF: $\mathrm{CH}_{2} \mathrm{Cl}_{2} 2.5: 1$, $0{ }^{\circ} \mathrm{C}$-rt, $15 \mathrm{~h}, 82 \%$ (c) $\mathrm{N}_{2} \mathrm{H}_{4} \cdot \mathrm{H}_{2} \mathrm{O}$ (4 eq), anhyd. EtOH, $50{ }^{\circ} \mathrm{C}, 5 \mathrm{~h}, 90 \%$

Figure 2.2 shows ${ }^{1} \mathrm{H}$ NMR stacked plots of the alcohol precursor 2.3 (Scheme 2.2) and QDA. $\mathrm{Br}$ that highlight the disappearance of the hydroxyl proton $(\mathrm{t}, \delta 4.99)$ as well as downfield shifts in the methylene protons of the aminooxy arm of QDA.Br. Product formation was also confirmed by high resolution mass spectrometry. 


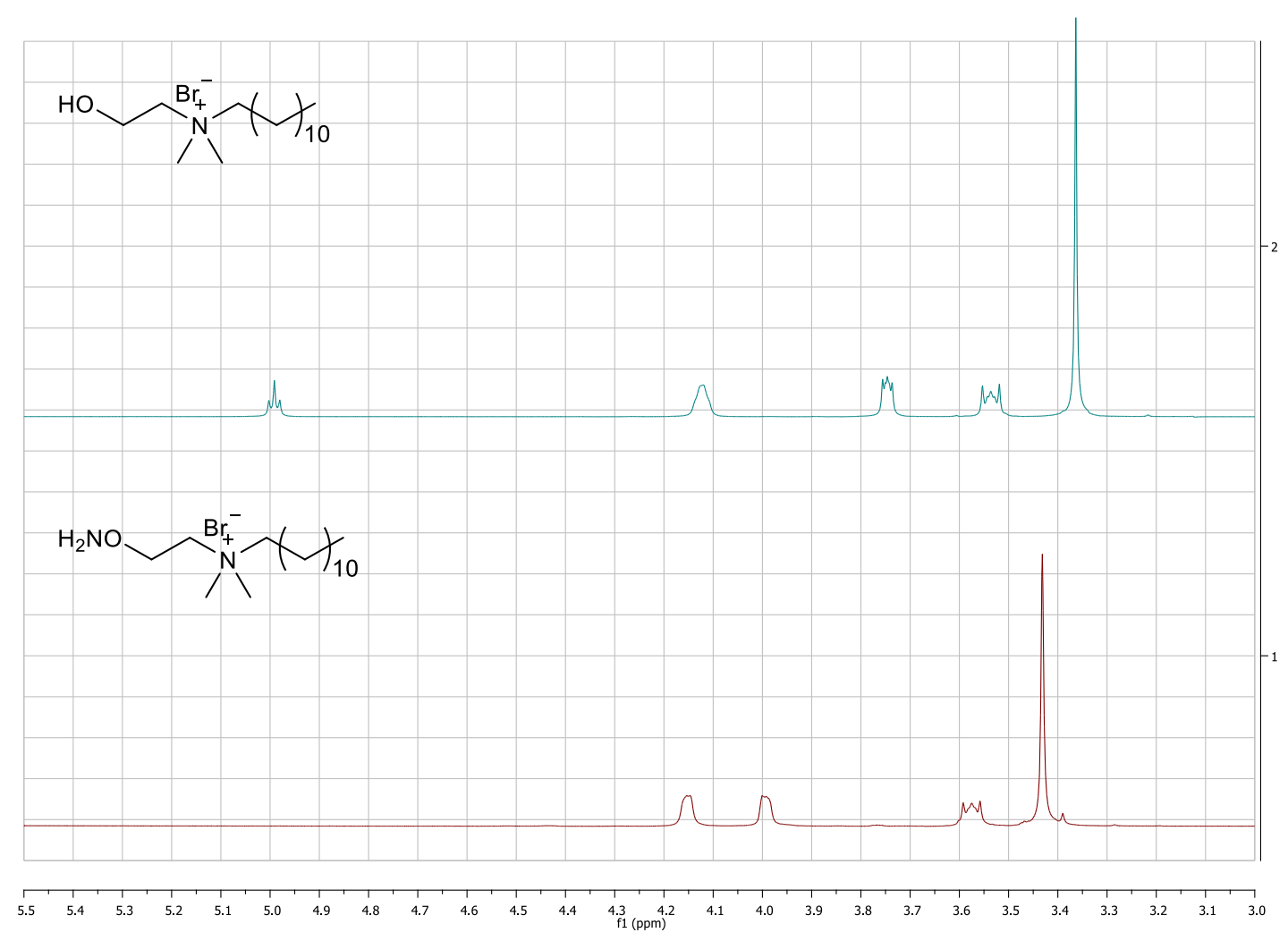

Figure 2.2. Stacked ${ }^{1} \mathrm{H}$ NMR spectra of alcohol 2.3 and QDA. $\mathrm{Br}$ in $\mathrm{CDCl}_{3}$

\subsubsection{Extraction solvent determination}

To compare the two analogs, QOA and QDA, and to determine the best solvent for extraction of adducts into an organic phase, each analog was reacted with a mixture of ketone and aldehyde standards (pyruvate, $\alpha$-ketoglutarate, $\alpha$-ketobutyrate, and glucose $^{1}$ ) in ammonium acetate buffer and subjected to extraction with one of three solvents: chloroform, ethyl acetate, or $n$-butanol. The ion intensities of the resultant conjugates, normalized to a standard of known concentration, were used to compare carbonyl capture efficiency between the two reagents (Figure 2.3). In both cases, $n$ -

\footnotetext{
${ }^{1}$ In the case of glucose, the aldehyde of the open-chain isomer is reactive with the reagent.
} 
butanol was the most efficient extraction solvent. In addition, QDA, the dodecyl reagent, proved superior at partitioning the standards into the organic phase as compared to the octyl version.
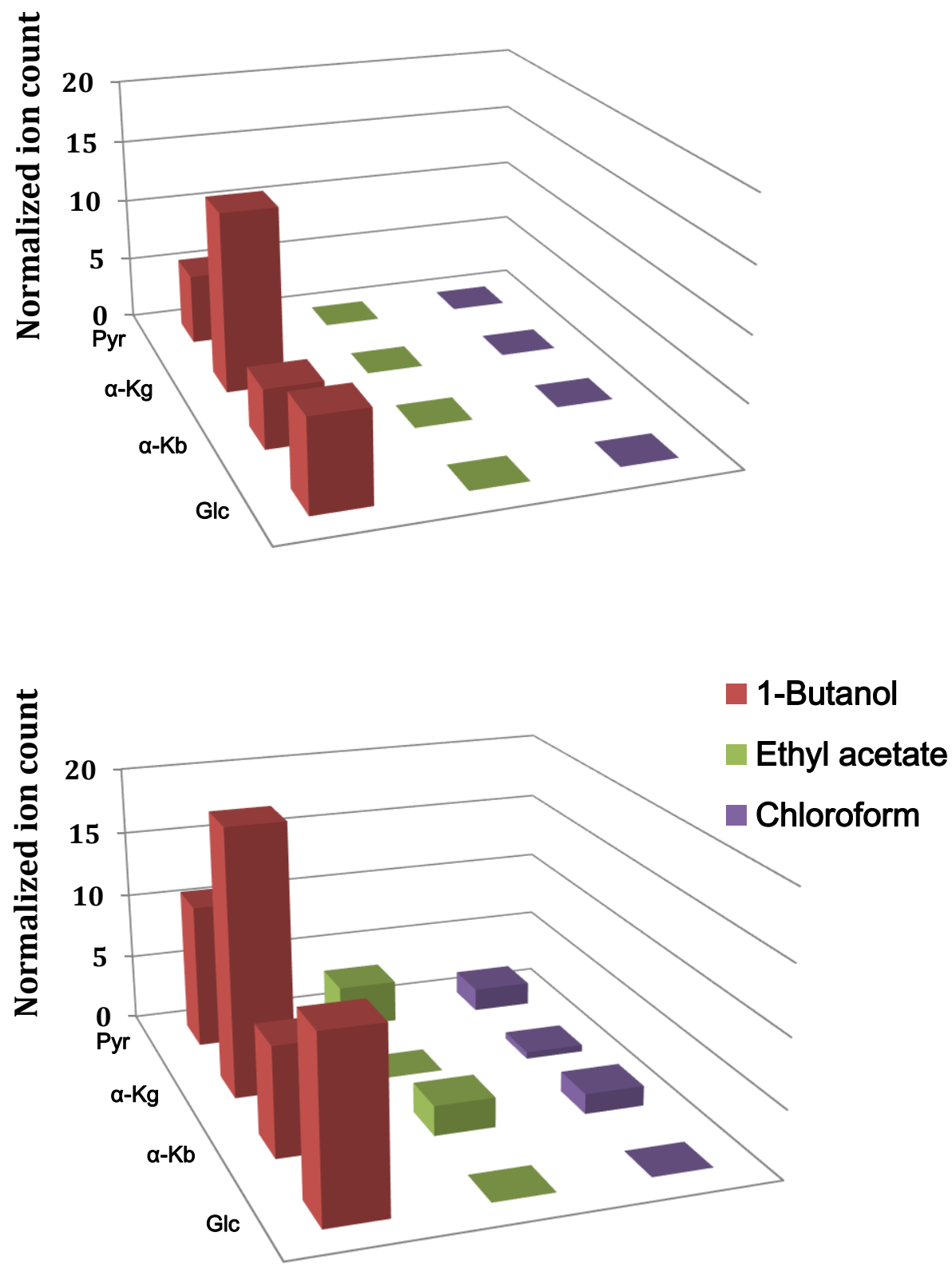

Figure 2.3. Comparison of normalized ion counts for adducts of QOA (upper) and QDA (lower) with standards pyruvate (Pyr), $\alpha$-ketoglutarate ( $\alpha$ $\mathrm{Kg}), \alpha$-ketobutyrate $(\alpha-\mathrm{Kb})$, and glucose (Glc) 


\subsubsection{Stable isotope labeled analog}

To prepare a stable isotope labeled version of QDA, we redesigned the original synthesis to allow for quaternization with a doubly labeled iodomethane, ${ }^{13} \mathrm{CD}_{3} \mathrm{l}$ (Scheme 2.3) and designated this analog * QDA. As the QDA previously synthesized (Scheme 2.2) had a bromide counterion, we prepared both unlabeled QDA iodide and *QDA iodide by this new route so that direct comparisons of the salts could be made. The successful incorporation of the label is apparent in Figure 2.4 in a mass spectrum of a mixture of the two salts showing a mass difference corresponding to $1{ }^{13} \mathrm{C}+3^{2} \mathrm{H}$.

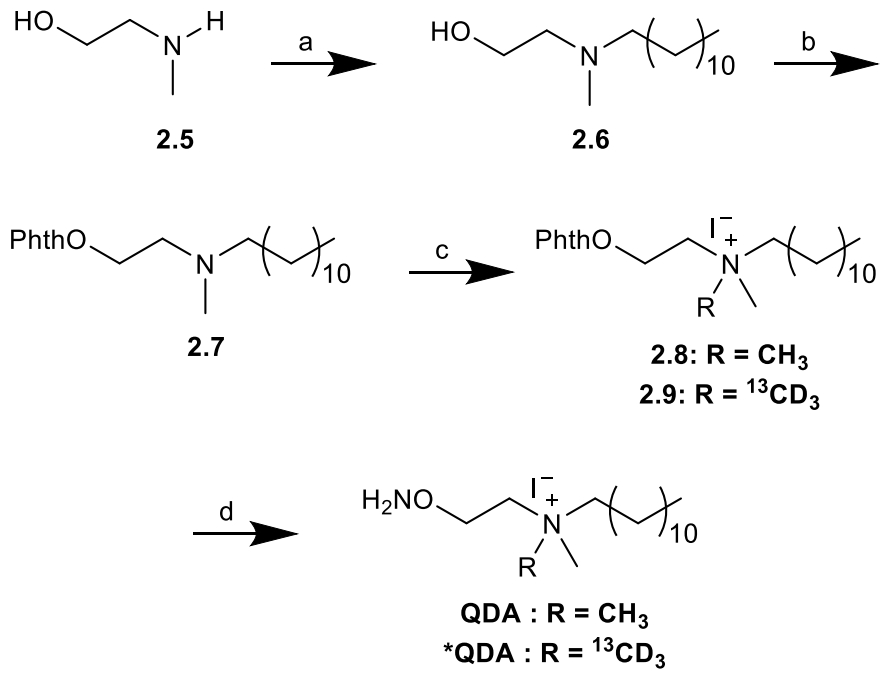

Scheme 2.3. Reagents and conditions: (a) 1-bromododecane (1.0 eq), anhydrous $\mathrm{Na}_{2} \mathrm{CO}_{3}(1.0 \mathrm{eq})$, EtOH, reflux, $20 \mathrm{~h} ; 70 \%$; (b) $\mathrm{N}$ hydroxyphthalimide (1.1 eq), $\mathrm{PPh}_{3}(1.1 \mathrm{eq}), \mathrm{DIAD}(1.1 \mathrm{eq}), \mathrm{THF}, \mathrm{rt}, 12 \mathrm{~h}$; 98\%; (c) $\mathrm{CH}_{3}$ l or ${ }^{13} \mathrm{CD}_{3} \mathrm{l}$ (2.5 eq), $\mathrm{CH}_{2} \mathrm{Cl}_{2}, 50{ }^{\circ} \mathrm{C}, 12 \mathrm{~h}$; (d) $\mathrm{N}_{2} \mathrm{H}_{4} \cdot \mathrm{H}_{2} \mathrm{O}$ (5.0 eq), anhydrous ethanol, rt (for QDA) or $50^{\circ} \mathrm{C}$ (for *QDA), $12 \mathrm{~h} ; 65-75 \%$ (2 steps). 


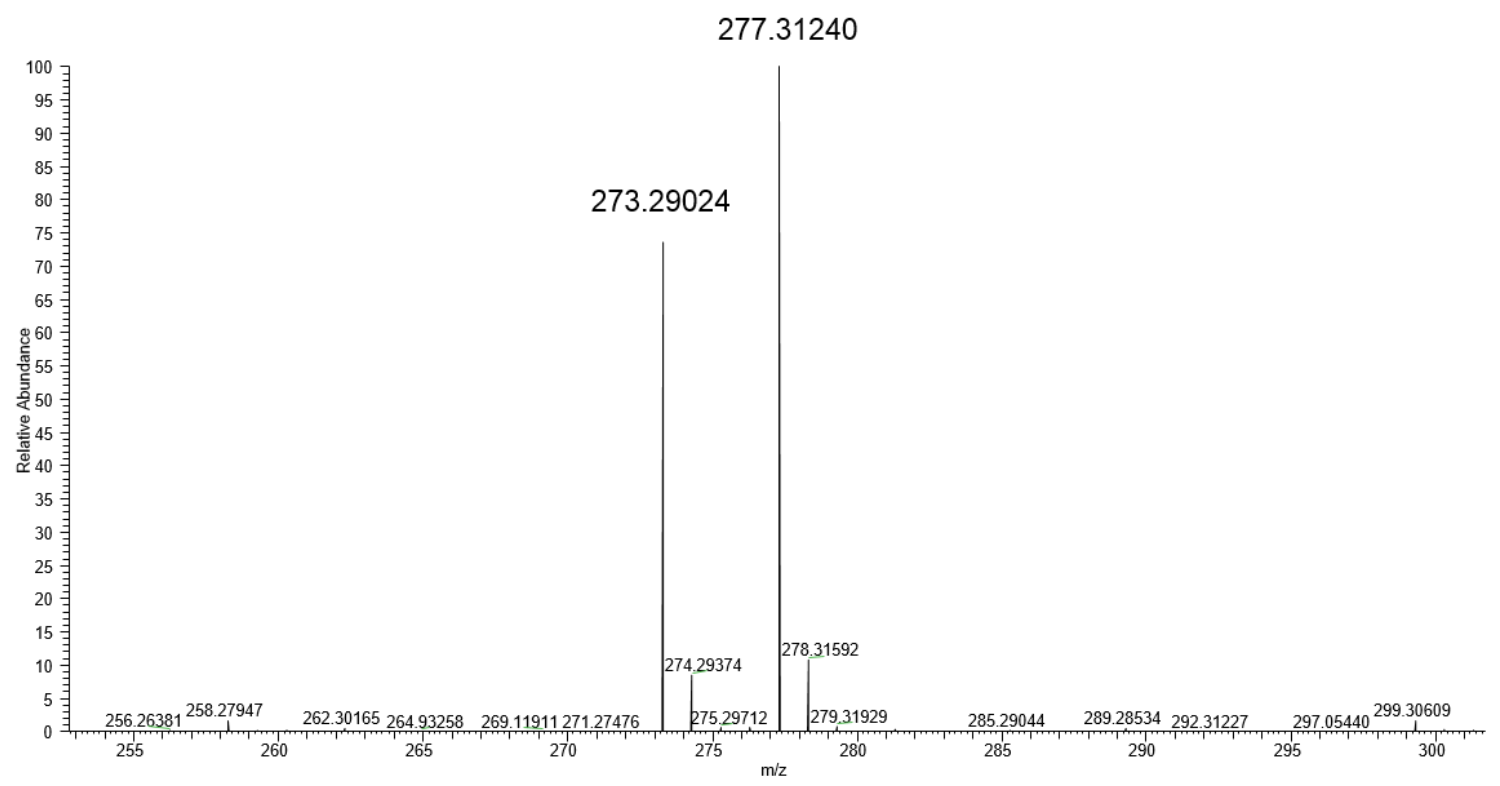

Figure 2.4. Mass spectrum of a 1:1 mixture of QDA.I and *QDA.I

\subsubsection{Counterion effects}

With analogs QDA bromide and *QDA iodide in hand, we examined the effect of the differing counterions by comparing MS signal intensity for derivatized mixtures of standards conjugated to each analog. Equal volume aliquots of the standards mixture described above were combined with solutions containing excess QDA bromide or * QDA iodide. The results, represented in Figure 2.5 , indicated a slight advantage for the iodo salt adducts, presumably through better partition of the larger ion pair into the organic layer during extraction. 


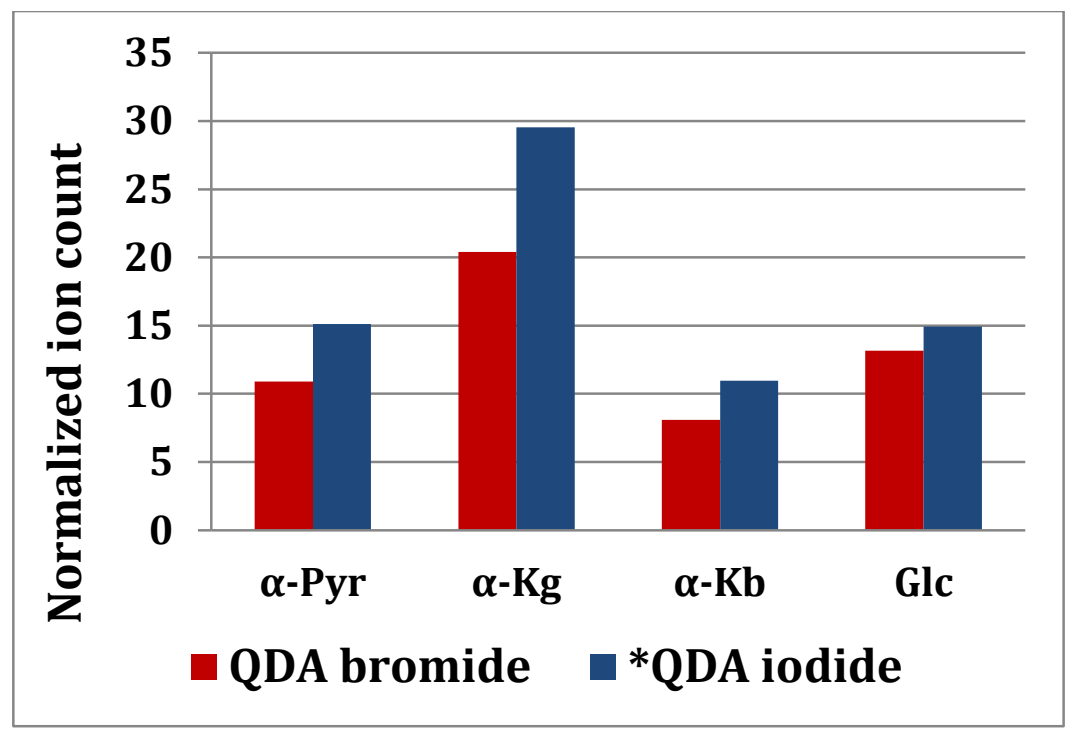

Figure 2.5. Comparison of QDA bromide and *QDA iodide salts on extraction of standards (single sample).

The efficiency of the partitioning process was also examined; we repeatedly extracted the same standard adducts from buffer into $n$-butanol five times. The comparison of normalized ion counts suggested that the majority of the adducts (ca. >98\%) were recovered within the first two extractions (Table 2.1). Therefore, in subsequent cellular extract studies we performed three solvent partitions of the derivatized extracts to ensure high adduct recovery. 


\begin{tabular}{|l|l|l|l|l|}
\hline Extraction & QDA-Pyruvate & $\begin{array}{l}\text { QDA- } \alpha- \\
\text { Ketoglutarate }\end{array}$ & $\begin{array}{l}\text { QDA- } \alpha- \\
\text { Ketobutyrate }\end{array}$ & QDA-Glucose \\
\hline 1 & 0.833 & 0.865 & 0.802 & 0.989 \\
\hline 2 & 0.155 & 0.118 & 0.196 & 0.011 \\
\hline 3 & 0.002 & 0.016 & 0.001 & 0.000 \\
\hline 4 & 0.009 & 0.000 & 0.000 & 0.000 \\
\hline 5 & 0.000 & 0.000 & 0.000 & 0.000 \\
\hline $\begin{array}{l}\text { Aqueous Layer } \\
\text { (post extraction) }\end{array}$ & 0.000 & 0.000 & 0.000 & 0.000 \\
\hline
\end{tabular}

Table 2.1. Fraction of total QDA-standard adducts partitioned per extraction into $n$-butanol from $5 \mathrm{mM}, \mathrm{pH} 6$ ammonium acetate buffer based on normalized ion count.

\subsubsection{Cell extract derivatization and analysis}

The partitioning of the constituents of lysed cells into a polar acetonitrile/water layer and a nonpolar chloroform layer is a common technique used for metabolite collection. Freeze-drying of the aqueous phase produces a cell extract that can be stored frozen and reconstituted when desired for analysis. These salty extracts are difficult to analyze by MS using traditional means; their high salt content can destabilize the electrospray, the size of the metabolites may fall below the more sensitive range of the MS (>150 $\mathrm{m} / \mathrm{z}$ ), and the ubiquity of anionic functionality (carboxylates, phosphates) often precludes detection by the more sensitive positive mode. As our custom reagent was designed to address these challenges, we set out to test its efficacy using the aqueous extracts of human lung adenocarcinoma A549 cells.

In a typical derivatization experiment, an aqueous solution of QDA was combined with a buffered solution of aqueous cell extract ( $\mathrm{pH} 6 \mathrm{NH}_{4} \mathrm{OAc}$ buffer). Mildly acidic $\mathrm{pH}$ facilitates oximation as loss of water is rate determining under such conditions. ${ }^{85}$ The mixture was then vortexed, sonicated, and placed in a $40{ }^{\circ} \mathrm{C}$ dry bath for 23 hours. This 
reaction time was chosen because increased ion counts were observed at up to $23 \mathrm{~h}$ of reaction time in $63 \%$ of the metabolites assigned, even though $85 \%$ of all assigned metabolites were observable after 30 minute of reaction time. In the case of reducing sugars, for example, the rate of ring opening will dictate the availability of the reactive aldehyde. Following reaction, the aqueous mixture was extracted with $n$-butanol three times, and dried by vacuum centrifugation. The resultant residue was dissolved in methanol with $0.1 \% \mathrm{v} / \mathrm{v}$ formic acid and analyzed by FT-ICR-MS as a $1: 1$ mixture with an external standard. Figure 2.6A and B show representative FT-ICR-MS spectra from such experiments. Figure $2.6 \mathrm{~A}$ represents a control condition, *QDA with a standards mixture; here, the adducts with the four standards are distinct. Figure 2.6B reflects QDAderivatized A549 cell extracts, where the QDA adducts of endogenous pyruvate, $\alpha$ ketobutyrate, $\alpha$-ketoglutarate, and glucose are readily observed, along with several other adducts. From the exact masses, the molecular formulae of additional observed adducts were assigned (see figure caption). 
A
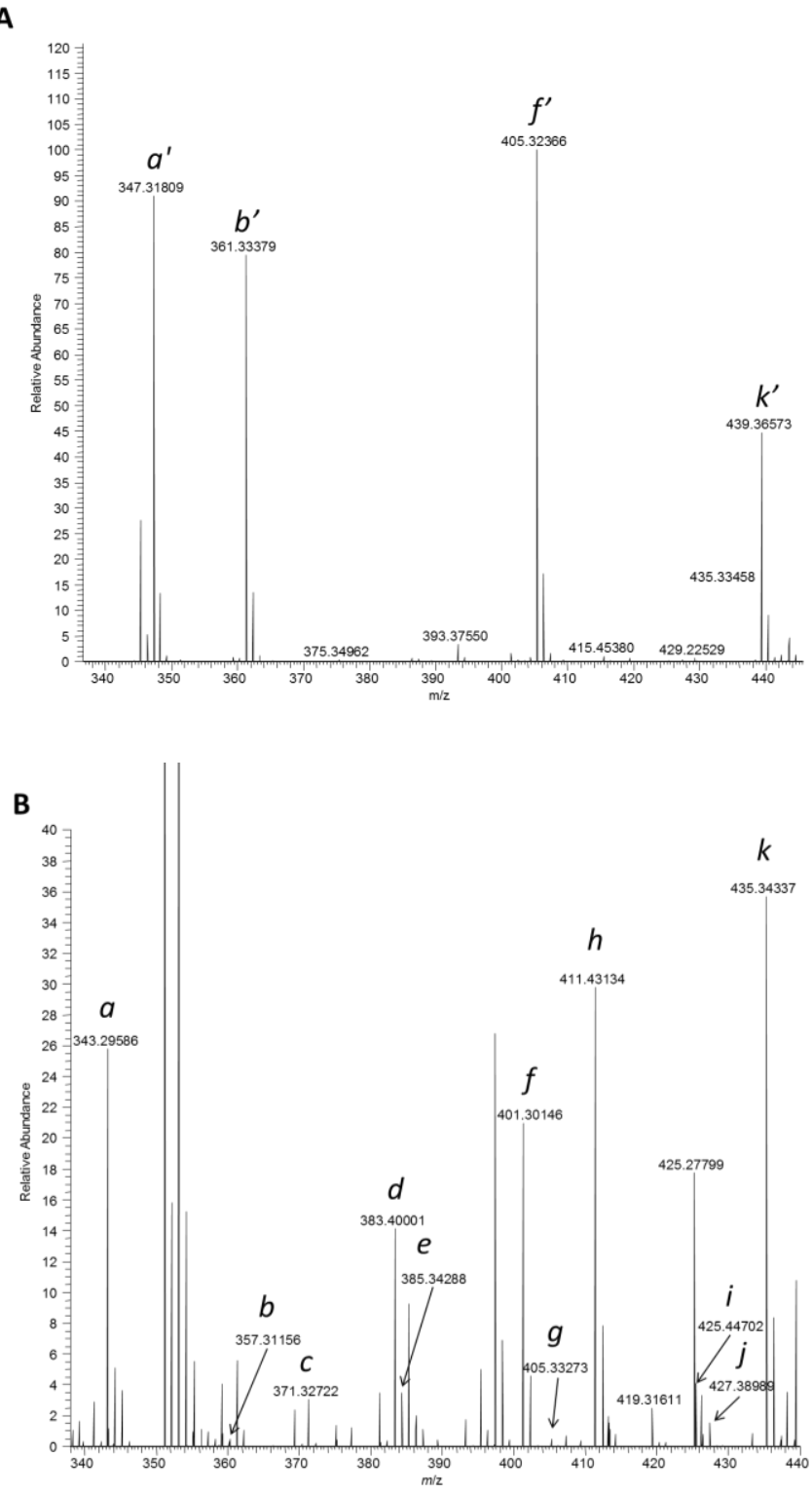

Figure 2.6. Derivatization of carbonyl standards and A549 cell extract. a) FT-ICR-MS spectrum of *QDA adducts of four carbonyl standards after partitioning from ammonium acetate buffer into $n$-butanol: $a^{\prime}=$ pyruvate, $b^{\prime}=\alpha$-ketobutyrate, $f^{\prime}=\alpha$-ketoglutarate, $k^{\prime}=$ glucose. b) FT-ICR-MS spectrum of QDA-derivatized A549 cell extract: $a=$ pyruvate, $b=\alpha$ ketobutyrate, $\mathrm{c}=\mathrm{C}_{7} \mathrm{H}_{14} \mathrm{O}, \mathrm{d}=\mathrm{C}_{8} \mathrm{H}_{16} \mathrm{O}$, e $=\mathrm{C}_{6} \mathrm{H}_{10} \mathrm{O}_{3}, \mathrm{f}=\alpha$-ketoglutarate, $\mathrm{g}=$ $\mathrm{C}_{5} \mathrm{H}_{10} \mathrm{O}_{5}, \mathrm{~h}=\mathrm{C}_{10} \mathrm{H}_{20} \mathrm{O}, \mathrm{i}=\mathrm{C}_{11} \mathrm{H}_{22} \mathrm{O}, \mathrm{j}=\mathrm{C}_{9} \mathrm{H}_{16} \mathrm{O}_{3}, \mathrm{k}=$ glucose 
$\mathrm{N}$-butanol was selected as an appropriate extraction solvent both for its high polarity and for the reportedly low partition of salts such as $\mathrm{NaCl}$ and $\mathrm{KCl}$ into the solvent (0.02-0.1 wt $\%$ at $\left.25^{\circ} \mathrm{C}\right) .{ }^{86}$ In fact, the partitioning of QDA adducts into $n$-butanol did appear to minimize the contribution of non-volatile salts in the final assay solution as confirmed by our finding that the sodiated peaks of the adducts of spiked carbonyl standards extracted from A549 cell extract represented, on average, only $0.1 \%$ of the total ion count $\left(\mathrm{H}^{+}+\mathrm{Na}^{+}\right)$for a given adduct.

\subsubsection{Metabolite identification}

As mentioned above, the value of the labeled analog, *QDA, is two-fold: first, derivatization using *QDA helps verify whether species observed in positive mode FT-ICRMS are genuine carbonyl adducts of QDA as opposed to other $n$-butanol-soluble cellular metabolites. "Sister signals" will be apparent in the case of a genuine adduct as a given pair of adducts represents a carbonyl metabolite that has reacted with both derivatizing agents. $^{29}, 87$ Second, isotopic labeling enables quantitative analysis of carbonyl metabolites in cell extracts, as will be demonstrated shortly for pyruvate, the product of glycolysis and the entry metabolite of the Krebs cycle.

Figure 2.7 illustrates how * QDA made possible the global visualization of carbonyl metabolites in a crude A549 cell extract. QDA and *QDA were added in equal amounts to an aqueous cell extract and carbonyl adducts were extracted as described above. This resulted in many pairs of adduct signals with comparable ion counts separated by 4.02188

$m / z\left(1 x^{13} \mathrm{C}+3 x^{2} \mathrm{H}\right)$. Example QDA and *QDA adduct pairs are delineated in Figure 2.7, 
where the full FT-ICR-MS spectrum (top inset) along with an expanded spectral region (bottom main panel) are shown. This spectrum was further processed to assign many likely carbonyl metabolites based on the derived molecular formulae of adducts matched against a custom database that compiles the molecular formulae of many known carbonyl metabolites. The adducts identified include $\alpha$-keto acids of the Krebs cycle (oxaloacetate and $\alpha$-ketoglutarate), carbohydrates (erythrose), lipid peroxidation products (eicosanal), serotonin metabolites (hydroxykynurenamine), nucleotide metabolites (deoxyribose), cofactors (pryidoxal-5-phosphate), and metabolites of glycolysis (pyruvate) and the pentose phosphate pathway (sedoheptulose). Table 2.2 is an abridged list of the QDA adducts assigned. Since structural isomers exist for many of these molecular formulae, the assignments shown here represent the most likely species based on known mammalian biochemistry. Further interrogation of these structural isomers by tandem MS and/or separation-based FT-ICR-MS methods is required to fully resolve their identity. 

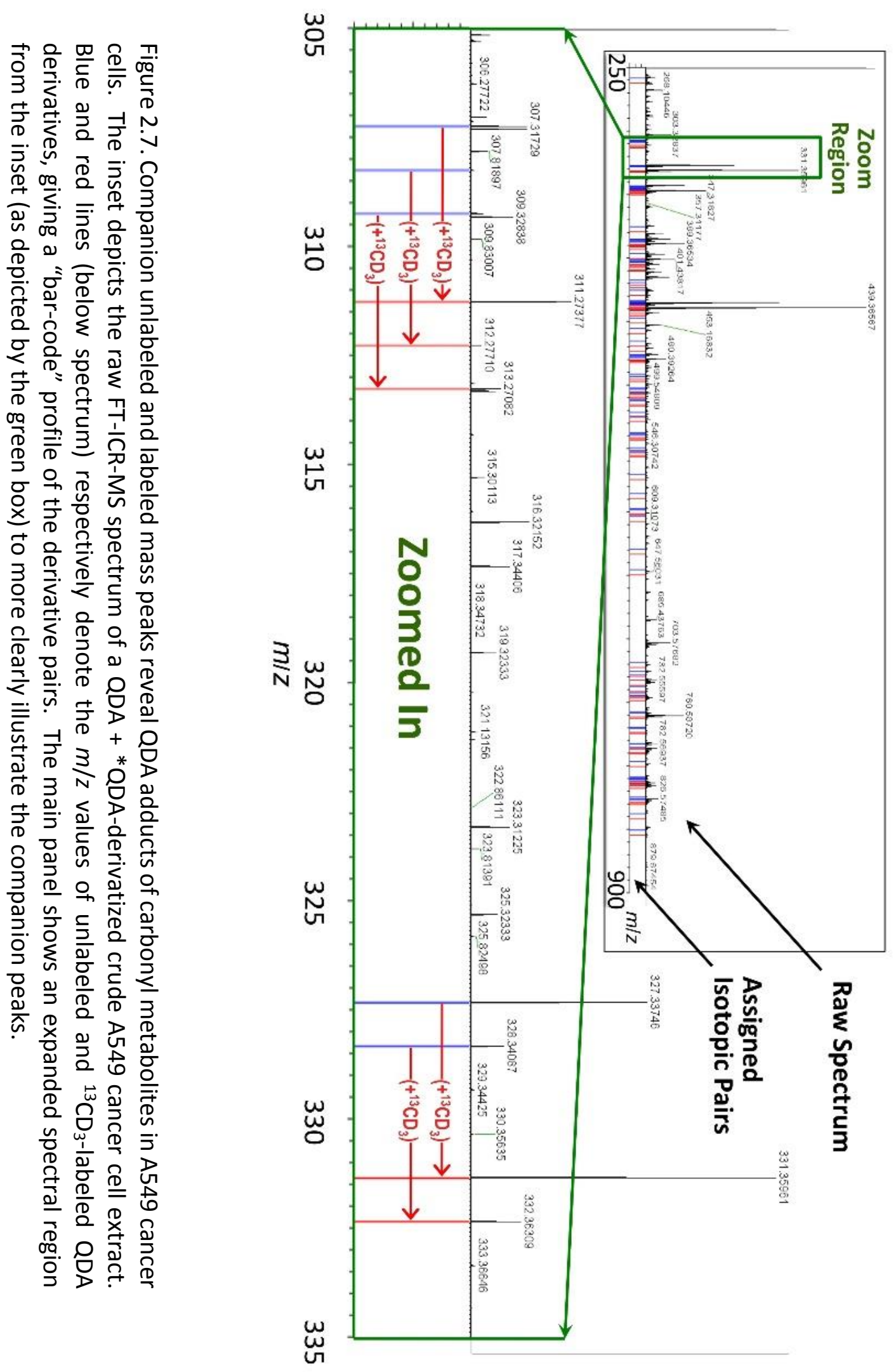
Table 2.2. Selected metabolites assigned based on Figure 2.7 spectral data

\begin{tabular}{|c|c|c|c|c|c|}
\hline Mass isomer assignments & $\begin{array}{l}\text { Molecular } \\
\text { formula }\end{array}$ & $\begin{array}{l}\text { Theoretical } \\
m / z \text { for QDA } \\
\text { adducts }\end{array}$ & Observed $m / z$ & Mass error & Ion count \\
\hline Pyruvate & $\mathrm{C}_{3} \mathrm{H}_{4} \mathrm{O}_{3}$ & 343.295519 & 343.295769 & 0.00025 & 905472.8 \\
\hline DHAP/GAP a & $\mathrm{C}_{3} \mathrm{H}_{7} \mathrm{O}_{6} \mathrm{P}$ & 425.277501 & 425.277999 & 0.000498 & 36810.4 \\
\hline Oxaloacetate & $\mathrm{C}_{4} \mathrm{H}_{4} \mathrm{O}_{5}$ & 387.285349 & 387.285619 & 0.00027 & 2621.8 \\
\hline D-Erythrose & $\mathrm{C}_{4} \mathrm{H}_{8} \mathrm{O}_{4}$ & 375.321734 & 375.322089 & 0.000355 & 22845.9 \\
\hline L-glutamate $\gamma$-semialdehyde & $\mathrm{C}_{5} \mathrm{H}_{9} \mathrm{NO}_{3}$ & 386.337718 & 386.338069 & 0.000351 & 33337.8 \\
\hline Deoxyribose & $\mathrm{C}_{5} \mathrm{H}_{10} \mathrm{O}_{4}$ & 389.337384 & 389.337689 & 0.000305 & 8659.2 \\
\hline$\alpha$-Ketoglutarate & $\mathrm{C}_{5} \mathrm{H}_{6} \mathrm{O}_{5}$ & 401.300999 & 401.301469 & 0.00047 & 193346.3 \\
\hline 5-Methylthio-D-ribose & $\mathrm{C}_{6} \mathrm{H}_{12} \mathrm{O}_{4} \mathrm{~S}$ & 435.325104 & 435.325029 & $-7.5 \mathrm{E}-05$ & 2982.9 \\
\hline Hexose (ketose and aldose) & $\mathrm{C}_{6} \mathrm{H}_{12} \mathrm{O}_{6}$ & 435.342864 & 435.343289 & 0.000425 & 3235226.3 \\
\hline Hydroxykynurenamine & $\mathrm{C}_{9} \mathrm{H}_{12} \mathrm{~N}_{2} \mathrm{O}_{2}$ & 435.369352 & 435.370609 & 0.001257 & 1106.1 \\
\hline Sedoheptulose & $\mathrm{C}_{7} \mathrm{H}_{14} \mathrm{O}_{7}$ & 465.353429 & 465.353969 & 0.00054 & 10502.8 \\
\hline Oxo-dodecanoic acid & $\mathrm{C}_{12} \mathrm{H}_{22} \mathrm{O}_{3}$ & 469.436369 & 469.437049 & 0.00068 & 33371.2 \\
\hline $\begin{array}{l}\text { S-(3-oxo-3-carboxy-n- } \\
\text { propyl)cysteine }\end{array}$ & $\mathrm{C}_{7} \mathrm{H}_{11} \mathrm{NO}_{5} \mathrm{~S}$ & 476.315268 & 476.316249 & 0.000981 & 1017.3 \\
\hline N-Acetylhexosamine & $\mathrm{C}_{8} \mathrm{H}_{15} \mathrm{NO}_{6}$ & 476.369413 & 476.370119 & 0.000706 & 291404.4 \\
\hline 5-isobutylthioribose & $\mathrm{C}_{9} \mathrm{H}_{18} \mathrm{O}_{4} \mathrm{~S}$ & 477.372054 & 477.373469 & 0.001415 & 77115.6 \\
\hline Pyridoxal 5-phosphate & $\mathrm{C}_{8} \mathrm{H}_{10} \mathrm{NO}_{6} \mathrm{P}$ & 502.30405 & 502.304809 & 0.000759 & 69620.1 \\
\hline Muramic acid & $\mathrm{C}_{9} \mathrm{H}_{17} \mathrm{NO}_{7}$ & 506.379978 & 506.380799 & 0.000821 & 3817.1 \\
\hline Octadecenal & $\mathrm{C}_{18} \mathrm{H}_{34} \mathrm{O}$ & 521.540439 & 521.541639 & 0.0012 & 1657.1 \\
\hline Eicosanal & $\mathrm{C}_{20} \mathrm{H}_{40} \mathrm{O}$ & 551.587389 & 551.588249 & 0.00086 & 26901.3 \\
\hline
\end{tabular}

a DHAP: dihydroxyacetone phosphate; GAP: glyceraldehyde-3-phosphate.

\subsubsection{Quantification of pyruvate}

An accurate quantification of carbonyl metabolites by the QDA method requires consideration of several factors. Among these are the linearity of instrumental response to concentration changes, the "ion suppression" (loss of signal intensity) due to the abundant charged species recovered from crude cell extracts, and the reaction and extraction efficiency of the derivatizing agent. 
We first verified linear instrumental response using a *QDA-derivatized standards mixture. Figure 2.8 , a scatter plot of ion counts versus concentration, illustrates that the FT-ICR-MS instrument response was linear in the range from 0.025 to $25 \mu \mathrm{M}$ for all four metabolite derivatives. Linear regression parameters are listed in Table 2.3.

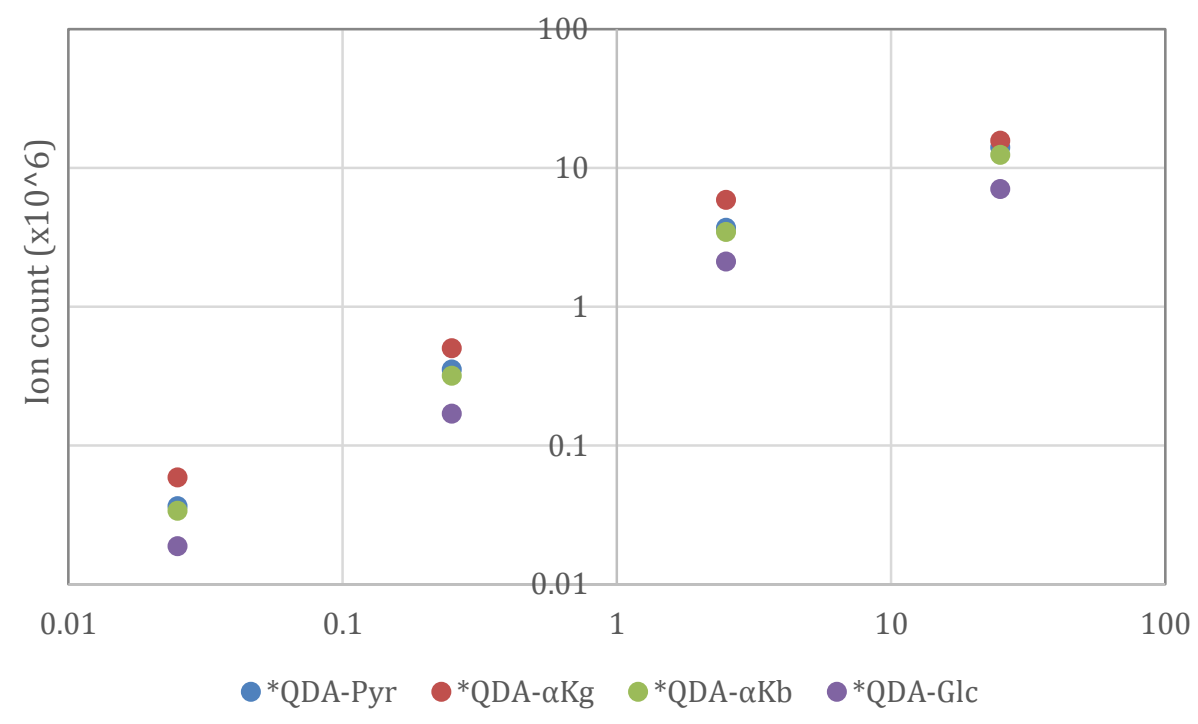

Figure 2.8. Log-log scatter plot of concentration versus ion count for four *QDA standard adducts. $\alpha \mathrm{Kg}=\alpha$-ketoglutarate; Pyr $=$ pyruvate $; \alpha \mathrm{Kb}=\alpha$ ketobutyrate; Glc = glucose.

\begin{tabular}{|l|l|l|l|l|}
\hline & ${ }^{*}$ QDA-Pyr & ${ }^{*}$ QDA- $\alpha$ Kg & ${ }^{*}$ QDA- $\alpha$ Kb & $*$ QDA-Glc \\
\hline Slope & 536847 & 579435 & 470851 & 265815 \\
\hline Intercept & 813724 & 1523225 & 781273 & 488369 \\
\hline RSQ & 0.97 & 0.92 & 0.97 & 0.96 \\
\hline
\end{tabular}

Table 2.3. Slopes, intercepts, and r-squared values for the linear regressions of Figure 2.6.

To correct for the effects of QDA reaction efficiency and adduct solvent partitioning efficiency, an internal standard of uniformly labeled ${ }^{13} \mathrm{C}_{3}$-pyruvate was spiked into cell extracts at a series of known concentrations prior to derivatization. For an 
estimate of the ion suppressing effects of the extract matrix, the ion counts of QDApyruvate (endogenous pyruvate) and $\mathrm{QDA}-{ }^{13} \mathrm{C}_{3}$-pyruvate (spiked) were normalized by dividing by the ion counts of an external standard, separately prepared *QDA-pyruvate, which was added at the same concentration to every sample. Figure 2.9 summarizes the results. As can be seen, the internal $\left[{ }^{13} C_{3}\right]$-pyruvate standard produced ion counts significantly higher for the mixture in buffer than for the mixture in cell extracts.

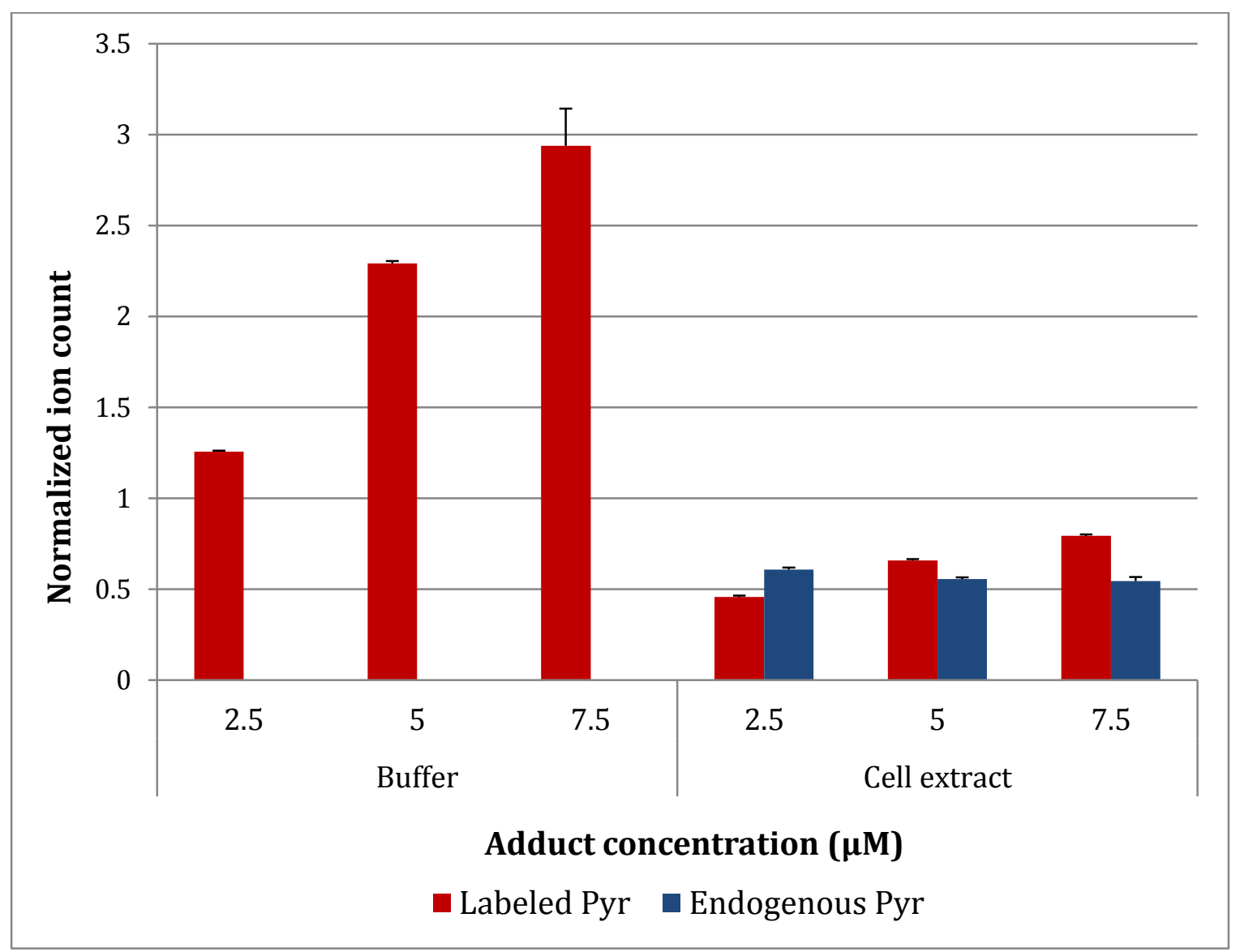

Figure 2.9. Uniformly ${ }^{13} \mathrm{C}$-labeled pyruvate spiked at three concentrations into either buffer (left bars) or cell extract (right bars) and derivatized in situ with excess QDA. Ion counts were normalized against that of an external standard of $2.5 \mu \mathrm{M} *$ QDA-pyruvate. Labeled Pyr $=\mathrm{QDA}-{ }^{13} \mathrm{C}_{3^{-}}$ pyruvate; endogenous Pyr = QDA-pyruvate. Error bars represent one standard deviation over three injections of a single sample. 
The normalized ion counts of QDA- ${ }^{13} \mathrm{C}_{3}$-pyruvate were then plotted against the known concentrations of $2.5,5$, and $7.5 \mu \mathrm{M}$ in buffer conditions and cell extract conditions, along with linear regressions (Figure 2.10). The ratio of the two slopes of the linear regressions indicated that there was a cellular extract ion suppression factor of 4.97 .

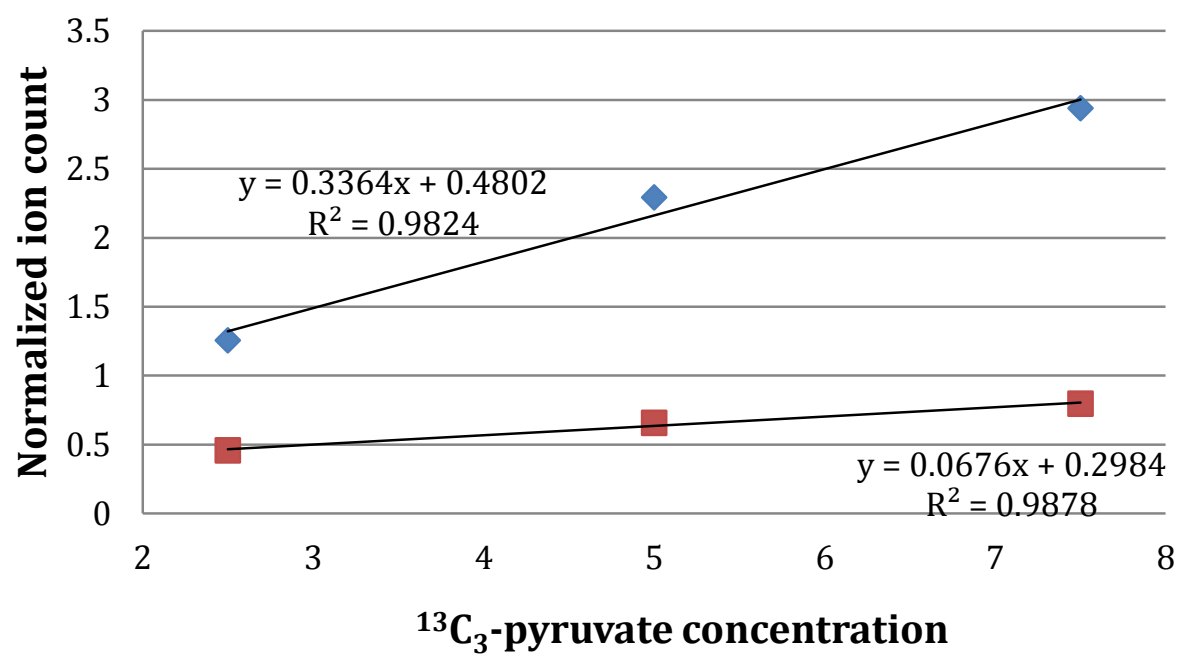

$\checkmark$ Adduct from buffer $\quad$ Adduct from cell extract

Figure 2.10. Scatter plot with linear regressions of the normalized ion counts of QDA- ${ }^{13} C_{3}$-pyruvate from buffer sample and cell extract sample at three concentrations, averaged over three injections.

Using these calibrations in a manner consistent with the stable isotope-based quantitation methodology commonly employed in quantitative proteomics, ${ }^{88}$ we estimated the pyruvate concentration to be 0.4 nanomoles in ca. 625,000 cells or approximately $106 \mathrm{nmol} / \mathrm{g}$ cell wet weight, when cell numbers were calibrated against cell wet weight. This result is within the reported concentration range for pyruvate in serum $(22-258 \mu \mathrm{M}){ }^{89}$ 


\subsection{Conclusions}

In summary, we developed a new cationic aminooxy reagent for the selective derivatization of carbonyl metabolites in aqueous cell extracts. QDA is sufficiently hydrophobic that its oxime ether adducts can be extracted efficiently into $n$-butanol for rapid analysis by FT-ICR-MS. Furthermore, we demonstrated a stable isotope labeling approach wherein *QDA and QDA were used to generate mass spectral ion pairs separated by $4.02188 \mathrm{~m} / \mathrm{z}$ for global profiling of carbonyl metabolites in crude extracts. The reagent could be easily adapted for multiplex analysis as the presence of two methyl groups permits the synthesis of 9 isotopologues using variably ${ }^{13} \mathrm{C} / \mathrm{D}$-labeled iodomethane. When applied to human lung cancer A549 cell extracts, numerous carbonyl metabolites across multiple structural classes were assigned, including important metabolites of the central metabolic pathways. Thus, this approach enables both high sample and information throughput analysis of key components of the overall cellular oxidation state. Lastly, this approach can facilitate quantification of these often lowabundant and labile metabolites, as exemplified by the analysis of cellular pyruvate. As such, our chemoselective derivatization approach coupled with high resolution and sensitive FT-ICR-MS analysis can overcome the difficult challenge of mapping key carbonyl metabolites as well as cellular oxidation states which may be indicators for the onset of diseases.

Since this work was disclosed in the journal Metabolomics in 2012, it has been expanded upon by other researchers in our group. A similar reagent with iodoacetimide functionality was developed for capture and profiling of thiols, for example, by my 
colleague Sadakatali Gori. We also continue to supply two groups of collaborators with the reagents QDA and *QDA, for additional profiling of cell extracts, and also for derivatizing human fluid samples at the University of Louisville's Diabetes and Obesity Center. The methodology for the use of QDA has been further optimized in studies by Dr. Pawel Lorkiewicz who has looked at how changes in $\mathrm{pH}$, temperature, and timing can achieve better reaction efficiency for specific classes of carbonyls.

Our Metabolomics article was cited by the researchers who developed the photocleavable linker for carbonyl capture described in Chapter $1 .{ }^{30}$ Their reagent incorporated an aminooxy group for carbonyl capture, but used biotin to sequester derivatives onto streptavidin beads for subsequent release by UV light. The critique that Yuan and coworkers had for our work was that their methodology detected 1784 carbonyl metabolites, whereas we listed only 31 . In fact, the QDA $/{ }^{*}$ QDA derivatization of cell extracts produced thousands of sister signals in the mass spectra. However, there is a difference between detection and assignment. We chose to only report those metabolites for which a specific chemical formula could be unequivocally assigned based on the detected exact mass. Additionally, we chose a very conservative threshold (1000 ion counts) to label a signal to be above the level of instrumental noise. As the work that we disclosed was only the proof of principle for the methodology, we are confident that the work being done by our collaborators on optimizing the reaction conditions and using a more sophisticated method for determining signal significance will lead to a broader and more comprehensive profile of carbonyl metabolites in biological samples. 


\section{CHAPTER 3 MAGNETIC NANOPARTICLES FOR AMF-MEDIATED DRUG DELIVERY}

3.1. Externally triggered release of drugs by AMF

3.2. Results and discussion

3.2.1. Preparation of iron oxide NPs

3.2.2. AMF heating properties

3.2.3. Fundamental study on the influence of hydroxyl substituents

3.2.4. Fluorescein release

3.2.5. Doxorubicin oxime ether analogs

3.2.6. AMF release profiles

3.2.7. In vitro studies

3.3. Sulfur-modified NPs

3.3.1. Dextran sulfate NPs

3.3.2. $\mathrm{CS}_{2}$-modified NPs

3.3.3. Greigite NPs 


\subsection{Externally triggered release of drugs by AMF}

The magnetic properties of iron oxide NPs described in chapter 1 highlight their potential for use in biological applications. Of particular interest to us was the phenomenon of inductive heating from exposure to an alternating magnetic field (AMF). Magnetic fluid hyperthermia, one of the most common medical application of ferrofluids, takes advantage of only the heating ability of NPs but fails to exploit their potential as carrier platforms for drugs, tracers, or targeting agents. Our intention in working with magnetic NPs, was to contribute to recent efforts focused on expanding NP applications to multipurpose therapies, and, in particular, to combine inductive heating with externally triggered drug delivery.

Typical NP formulations designed for AMF-triggered release of bound substrates rely upon a thermally labile mediator. For example, when NPs are associated with liposomes, ${ }^{90-91}$ lipid coatings, ${ }^{92}$ or solid lipid particles, ${ }^{93}$ temperature elevation triggers changes in lipid packing and permeability which become the basis for substrate release. Otherwise, thermosensitive polymers, gels, ${ }^{94}$ and macrocyclic "nanovalves" 95 have also been used for heat-gated release of drugs from NP platforms.

In developing reagents for external triggering, we aimed for a more straightforward approach, one in which a drug could be directly bound through an optimized linker to a NP via electrostatic interactions or hydrogen bonding. We pursued a binding motif that would be strong enough to stabilize the attachment of a drug onto the NP surface, but weak enough for AMF-mediated release. To explore the concept, we prepared a panel of aminooxy functionalized quaternary ammonium salts for attachment 
to a NP surface and, using a fluorophore, probed its AMF release properties. This led us to do a more fundamental study of the influence of hydroxyl groups and counterions on the binding of organic molecules to NPs. We then prepared a Dox analog for reversible attachment to NPs and looked at its release properties as well as its efficacy in vitro. Inconsistencies in the results led us to consider modifications to the NP surface to facilitate stronger binding of Dox analogs. A brief exploration of sulfur modified NPs yielded data that were revealing of the complexity of the iron oxide surface and peculiarities at the nanoscale.

\subsection{Results and discussion}

\subsubsection{Preparation of iron oxide NPs}

To synthesize iron oxide NPs, we followed a well-established ${ }^{36,96-97}$ wet chemistry approach based on the coprecipitation of iron II and III chloride salts in aqueous base as described by Mikhaylova et al. ${ }^{98}$ Transmission electron microscopy (TEM) imaging ${ }^{99}$ of particles prepared using this method revealed particle diameters of 5-10 nM. Zeta potential measurements, which measure the electrophoretic mobility of charged colloidal suspensions, showed a surface charge of $\approx-32 \mathrm{mV}$.

Whereas the coprecipitation method intends to produce the magnetite phase of iron oxide crystal $\left(\mathrm{Fe}_{3} \mathrm{O}_{4}\right)$, it is also well documented that exposure to air and moisture can lead to oxidation and conversion to $\mathrm{y}-\mathrm{Fe}_{2} \mathrm{O}_{3}$ particles. ${ }^{100-102}$ Washing of the freshly prepared nanoparticle precipitate with a dilute hydrochloric acid solution is recommended $^{97}$ in order to peptize the particles and form a stable colloid and it is 
documented that avoiding this step tends to prevent colloid formation in aqueous media unless a hydrophilic coating species is introduced in its place. ${ }^{36}$ We reasoned that rinsing of the freshly prepared nanoparticle precipitate was likely sufficient to cause substantial oxidation. Moreover, we found that depending upon how the particles were handled after precipitation, there were batch-to-batch inconsistencies in the color, texture and magnetoviscocity of the resultant NPs. Color changes can be indicative of crystal phase changes, ${ }^{100}$ so we examined the phase of magnetite generated by our synthetic and workup procedures. Crystallography and Mössbauer spectroscopy are commonly employed to differentiate the two phases, however the data generated from these methods can be difficult to interpret as the crystal structures of the two phases are similar, and nanoscale properties may differ from those of the bulk crystal. ${ }^{103}$ This point is illustrated in Figure 3.1 which depicts a Mössbauer spectrum of a sample of our NPs acquired by Dr. Charles Johnson from the University of Tennessee Space Institute. The black lines (experimental data) are overlayed with reference peaks (red and green lines) that distinguish the subtle chemical shift differences for $\mathrm{Fe}^{3+}$ in octahedral versus tetrahedral coordination. 


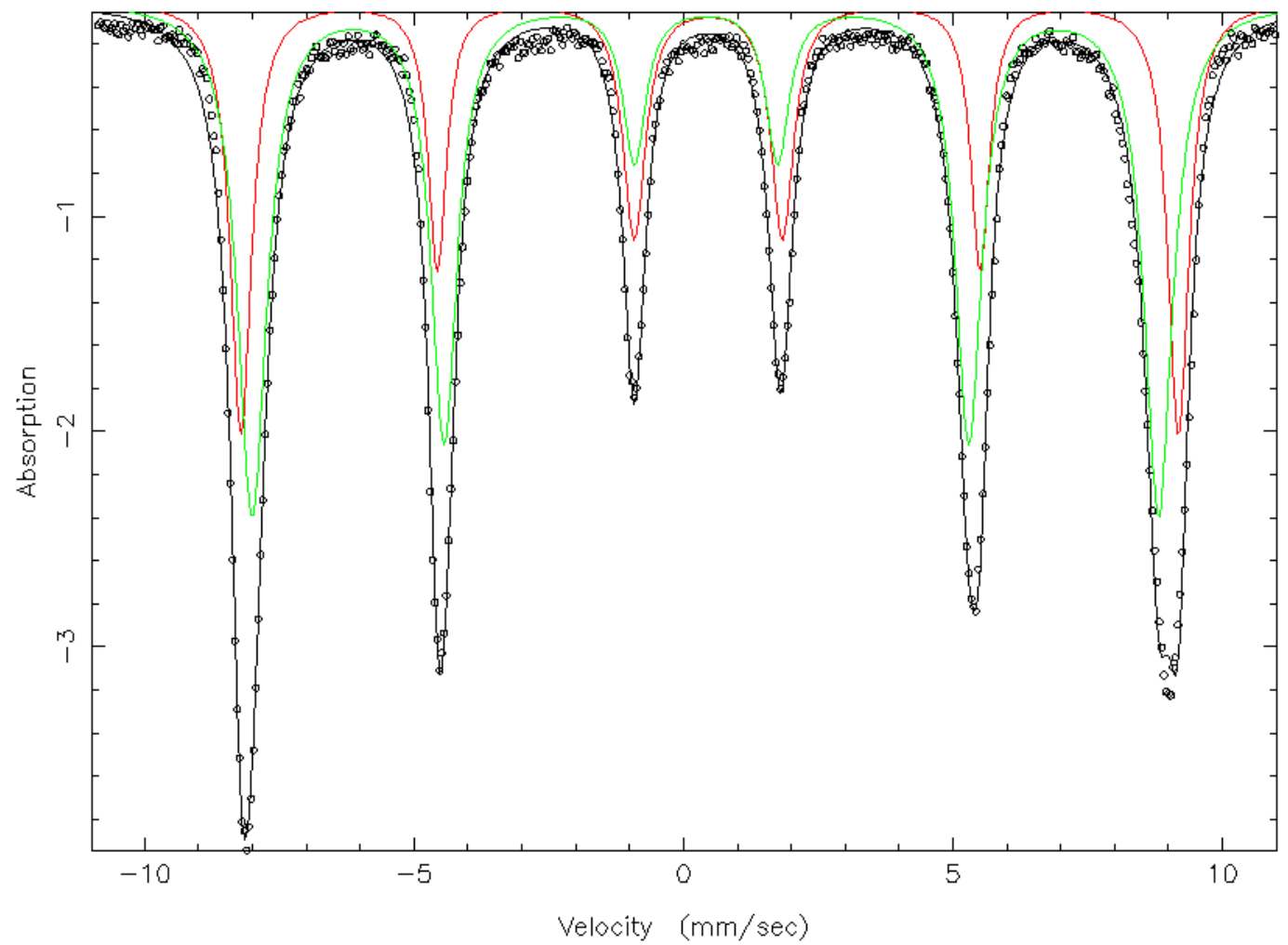

Figure 3.1. Mössbauer spectrum of NPs prepared by coprecipitation

We quantified the relative proportions of the two crystal phases in our NPs through a spectrophotometric method ${ }^{104}$ developed by Amonette and Templeton ${ }^{105}$ that enables the quantification of $\mathrm{Fe}^{2+}$ present exclusively in the $\mathrm{Fe}_{3} \mathrm{O}_{4}$ phase. During chemical analysis the synthesized NPs were dissolved in an acid matrix and complexed with iron II chelator 1,10-phenanthroline. Absorbance at $510 \mathrm{~nm}$ of the digestate allowed quantification of $\mathrm{Fe}^{2+}$. Further, reduction of $\mathrm{Fe}^{3+}$ in the NP sample using hydroxylamine allowed for a total Fe determination. Our results for the assay indicated that during the surface acidification work-up phase dictated by the Mikhaylova procedure, the amount of $\mathrm{Fe}^{2+}$ decreased and the amount of $\mathrm{Fe}^{3+}$ increased indicating over-oxidation. For comparison, particles that were washed with neutral water alone were closer in 
composition to stoichiometric $\mathrm{Fe}_{3} \mathrm{O}_{4}$. (Table 3.1). In either case, our characterization established the prepared products as a mixture of $\mathrm{Fe}_{2} \mathrm{O}_{3}$ and $\mathrm{Fe}_{3} \mathrm{O}_{4}$ phases. And indeed many investigators acknowledge this phenomenon and choose to report their preparations as a combination of the two phases. ${ }^{101,106}$

\begin{tabular}{|l|l|l|}
\hline & $\mathrm{Fe}^{2+}$ (weight \%) & $\mathrm{Fe}^{3+}$ (weight \%) \\
\hline Neutral rinse & 14.8 & 55.6 \\
\hline Acid rinse & 7.7 & 64.8 \\
\hline Ref. for stoichiometric $\mathrm{Fe}_{3} \mathrm{O}_{4}$ & 24.1 & 48.24 \\
\hline
\end{tabular}

Table 3.1. Effect of work-up conditions on Fe composition

We also observed a second effect when rinsing NPs, namely neutralization of surface charge. Figure 3.2 shows that NPs exposed to a single neutral rinse remain anionic, whereas multiple rinses generate neutral particles, and acid rinses produce highly positive NPs. Given the greater magnetic susceptibility of $\mathrm{Fe}_{3} \mathrm{O}_{4}$ as compared to $\mathrm{Fe}_{2} \mathrm{O}_{3}$, and the better charge pairing of anionic NPs with cationic ammonium salts, we chose to continue our work with NPs prepared without surface acidification. 


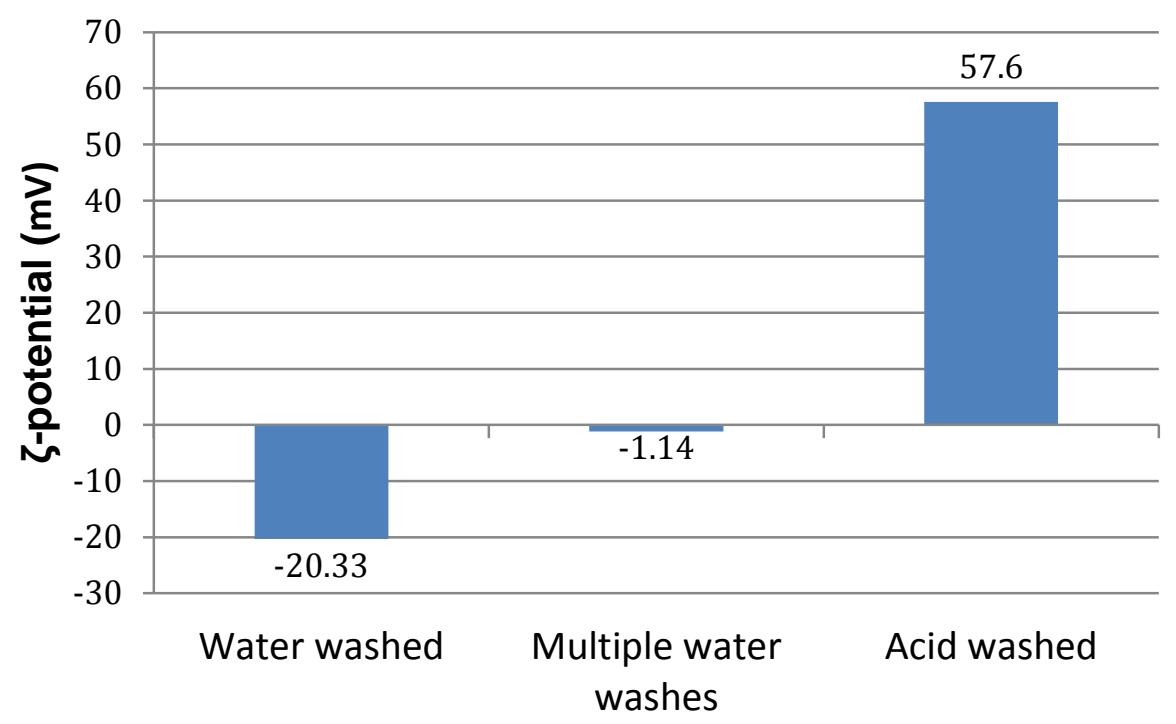

Figure 3.2. 3-potential measurements of NPs under three work-up conditions

\subsubsection{AMF heating properties}

To test the potential of our NPs for heating in the presence of an AMF, several concentrations of uncoated NPs were prepared in water with $3 \%$ alginate. The rationale for the use of alginate was twofold; first, the bare particles are poorly dispersible in water, so the alginate provides increased viscosity to maintain suspension, and secondly, electrolytes are necessary for effective NP-mediated heating of the bulk solution. The aqueous NP solutions were exposed to AMF for up to 60 minutes while temperature measurements were made with a fiber optic probe. As can be seen in Figure 3.3, solution heating was dependent upon NP concentration, and $5 \mathrm{mg} \mathrm{NP} / \mathrm{mL}$ was sufficient to raise the temperature of the surrounding solution by $10^{\circ} \mathrm{C}$. Hyperthermia therapy, by comparison, requires temperature increases of $5-8^{\circ} \mathrm{C} .{ }^{107}$ 


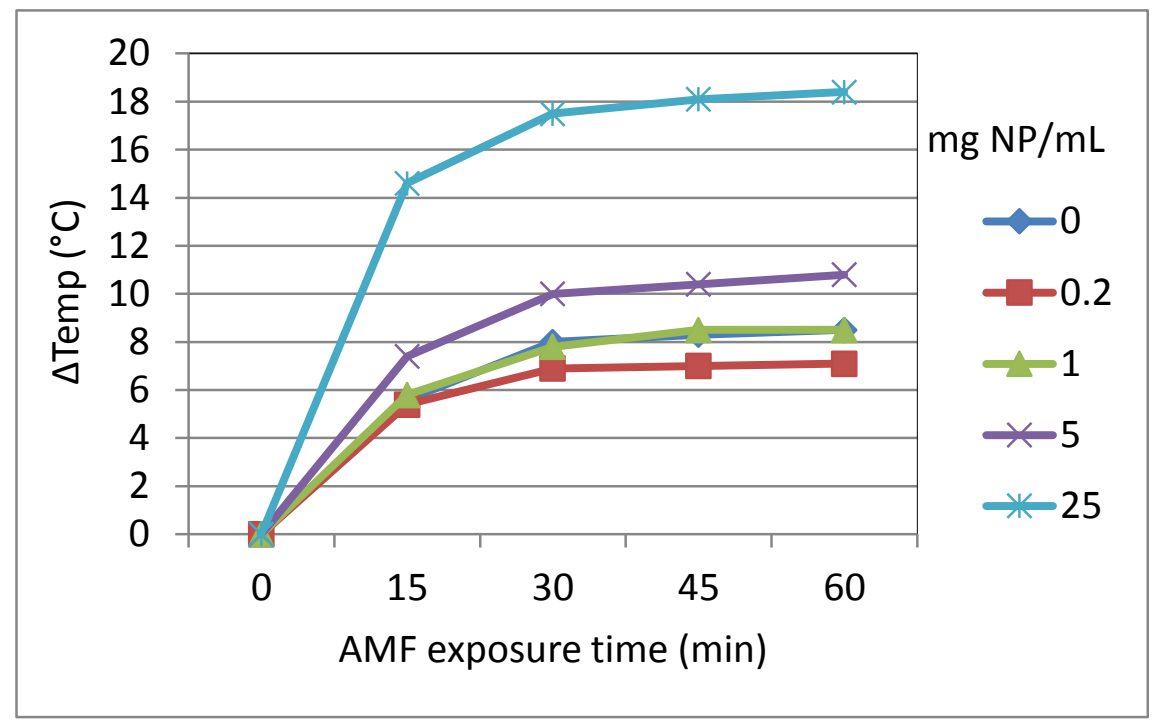

Figure 3.3. Change in temperature of water with $3 \%$ alginate during AMF exposure of $201 \mathrm{~A}, 203 \mathrm{kHz}$

\subsubsection{Fundamental study on the influence of hydroxyl substituents}

Our rationale for preparing anionic NPs was ultimately to utilize their charge properties to noncovalently bind a pharmaceutical agent. In light of this goal, we focused on developing agent-linkers based on positively charged ammonium salts (i.e., prodrug modifiers) to promote electrostatic interactions with the negatively charged NP surface. Secondly, given that the surface of iron oxide supports oxyanions, ${ }^{59}$ we also wanted to demonstrate that hydrogen bonding groups could promote binding of small molecules to NPs. To systematically investigate the binding impact of hydroxyl substituents, we synthesized a panel of ammonium salts (and for comparison, a free amine) which varied by the number and arrangement of hydroxyethyl groups (Figure 3.4). The salts were prepared by alkylation of tertiary ammonium precursors with iodomethane, generating iodo salts. 


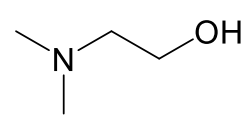

Amine

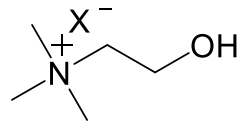

$1 \mathrm{OH}$

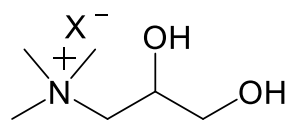

1,2 diol

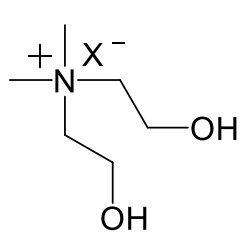

$2 \mathrm{OH}$

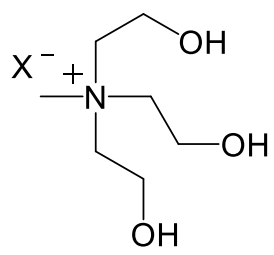

$3 \mathrm{OH}$ $\mathrm{X}=\mathrm{Cl}, \mathrm{I}$

Figure 3.4. Panel of variably hydroxylated ammonium salts

We also were interested to see the effect of varying the counterion on binding properties, so the chloride forms of each salt were prepared through ion exchange methodology. Exchange of iodide for chloride produced little change in the NMR of the compounds, however exchange was verified in two other ways. In the first method, silver nitrate was added to aqueous solutions of the ammonium salts and the color of the precipitated silver halide was observed; silver chloride forms white crystals, whereas silver iodide forms yellow crystals. MS was also used to verify anion exchange by looking for a signal representing the mass of dimers of the salts ( $m=2$ ammonium cations +1 halide, net charge $=+1$ ). For example, the mass of $10 \mathrm{H} \cdot \mathrm{Cl}$ was calculated at $\mathrm{m} / \mathrm{z}$ 243.18283 and observed at 243.18357, but no iodide dimer was detected.

To quantify the amount of organic material bound to ammonium coated NPs, thermogravimetric analysis (TGA) was used. The precise weight of the NP sample was monitored while the sample was heated to $700{ }^{\circ} \mathrm{C}$. Sample weight loss below $200{ }^{\circ} \mathrm{C}$ was attributed to adsorbed water ${ }^{108}$ and above $200{ }^{\circ} \mathrm{C}$ was assigned to the desorption of bound organic molecules. 


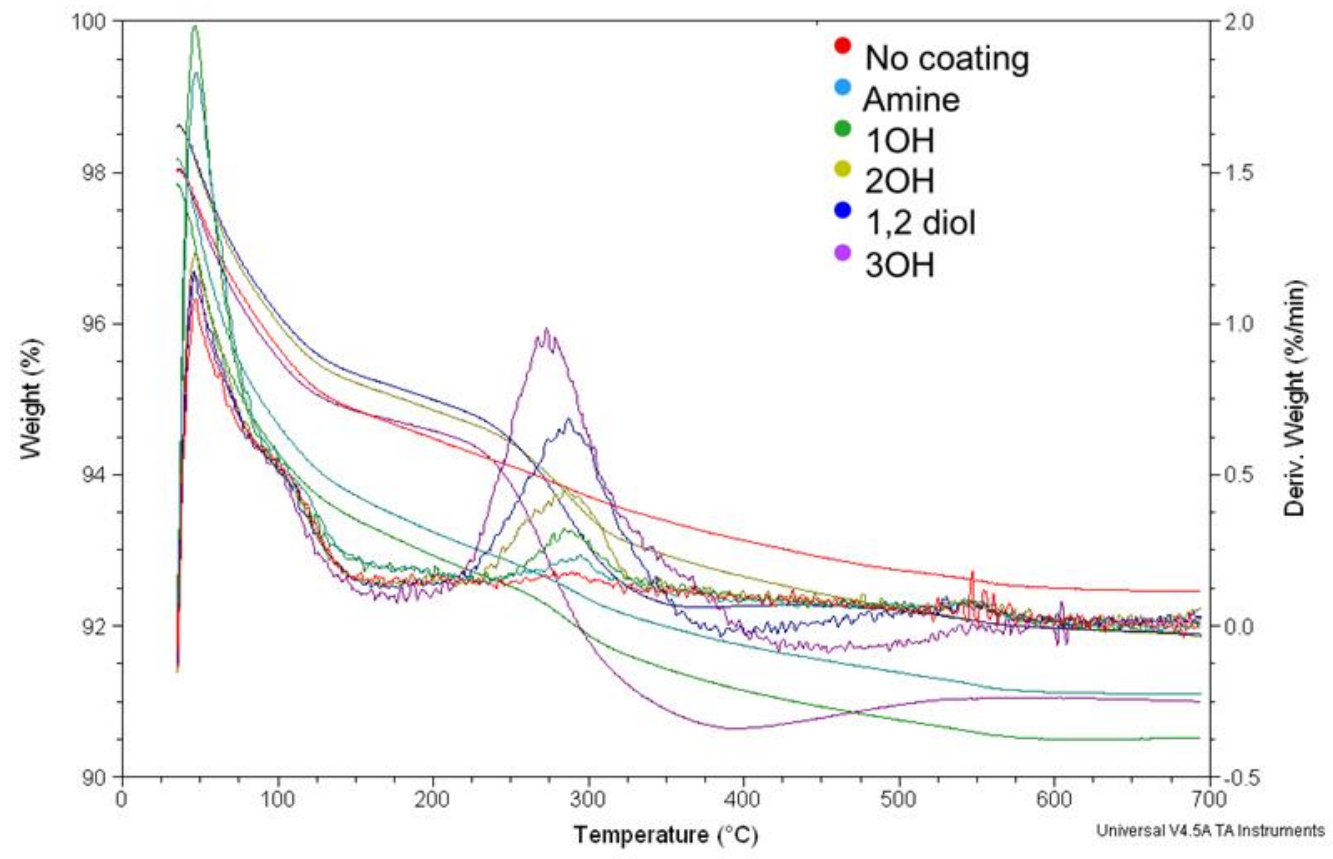

Figure 3.5. A representative TGA of $\approx 6 \mathrm{mg}$ each of NPs coated with variably hydroxylated ammonium salts (iodide form) and free base amine

The results of the TGA study supported our hypothesis that an ammonium ion motif having hydroxyl substituents would enhance binding to anionic NPs through both electrostatic and hydrogen bonding interactions at the NP interface. A representative TGA depicting the iodo salts can be seen in Figure 3.5; descending lines represent actual percent weight loss and, for clarity, the derivatives of those lines are overlaid. Figure 3.6 defines the number of bound organic molecules, calculated according to equations supplied by Galeotti et al., ${ }^{109}$ on each of the coated NP formulations. There was enhanced binding of ammonium salts when more hydroxyethyl substituents were present. Additionally, the chloride salts proved to be more efficient binders than the iodide salts. This may have resulted because ion pairs of quaternary ammonium cations with chloride 
are weaker than their iodide counterparts; ${ }^{110}$ thus displacement of the counter-ion by NP surface anions may be more readily achieved for the chloride salts.

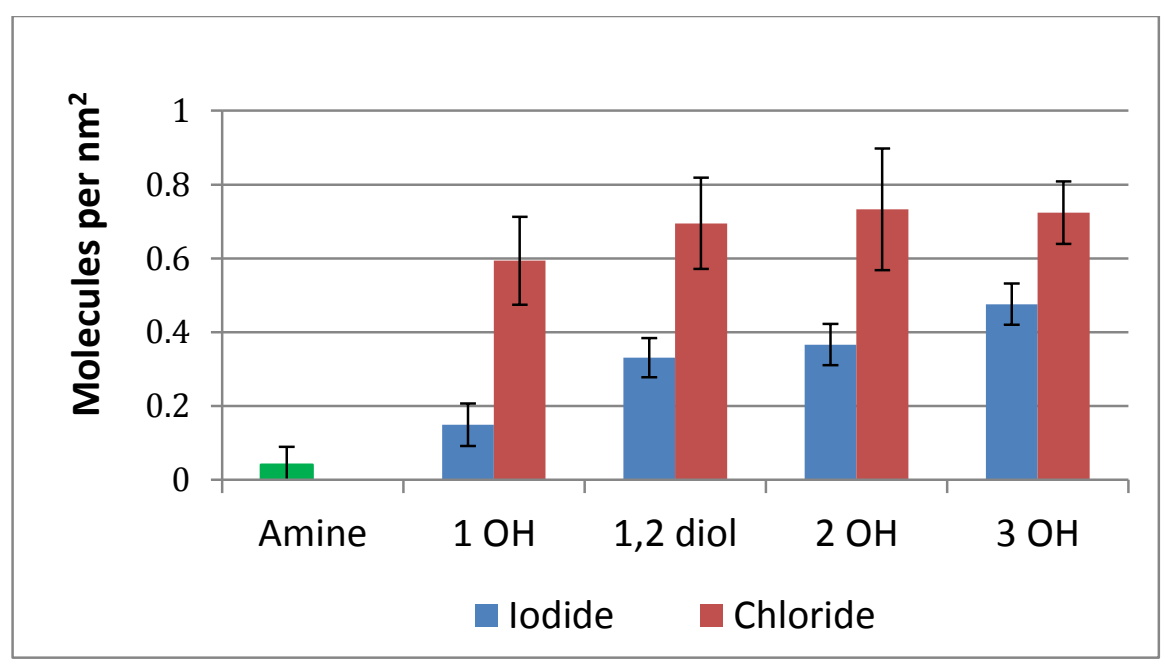

Figure 3.6. TGA results of bound panel members (see Figure 3.4 for structures).

\subsubsection{Fluorescein release}

From our fundamental study we understood that hydroxyl groups aid in the binding of small molecules to NPs. As our ultimate goal was AMF mediated release of NP bound substrates, we needed to determine the degree of binding that would cause ammoniumbased linkers to adhere tightly to NPs under normal conditions, but release from the particles under AMF exposure. A fluorescent probe was chosen for this purpose as it would enable spectroscopic monitoring of release as well as function in the capacity of a drug mimic. To this end, fluorescein isothiocyante (FITC) was modified as shown in Scheme 3.1 to install an aldehyde functional group for ease of conjugation with aminooxy functionality. 


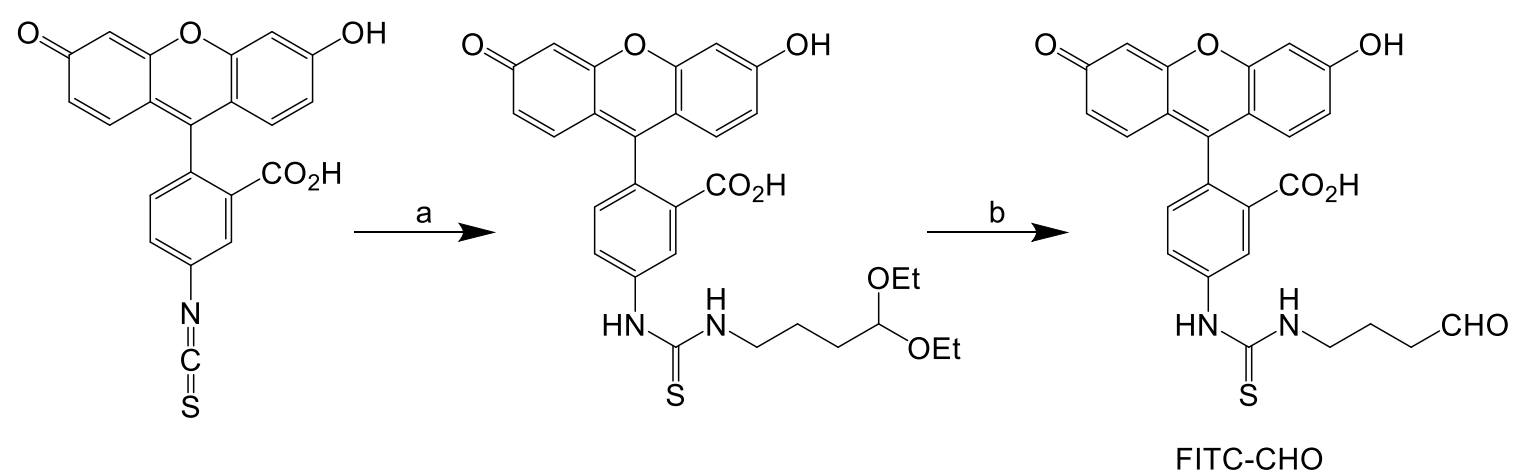

Scheme 3.1. Reagents and conditions: (a) 4-aminobutyraldehyde diethylacetal (1.2 eq), dimethyl-formamide, rt, $4 \mathrm{~h}$ (b) $30 \%$ aq. acetic acid, $3 \mathrm{~h}, 55 \%$ (2 step)

Next, three quaternary aminooxy (QAO) salts (Figure 3.7) were prepared for conjugation with the FITC aldehyde (FITC-CHO). The first, designated QAO, was prepared by phthalimide protection of the alcohol dimethylethanolamine. Methylation with iodomethane produced the quaternary amine and hydrazinolysis cleaved the phthalimide to an aminooxy. QAO-2OH was prepared by first protecting two of the alcohols on triethanolamine with silyl groups, then proceeding with the phthalimide protection and methylation as before, and finally deprotecting the alcohols to achieve the final bishydroxy product (Scheme 3.2). $\mathrm{A}^{1} \mathrm{H}$ NMR expansion showing the methylene protons of QAO-2OH reveals the 4:2 integrations anticipated for the difunctional molecule (Figure 3.8). QAO-OH was prepared by my colleague Xuan Huang. ${ }^{111}$ 

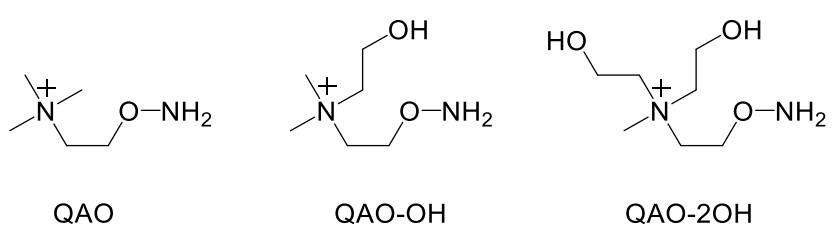

Figure 3.7. QAOs with variable hydroxyl substituents

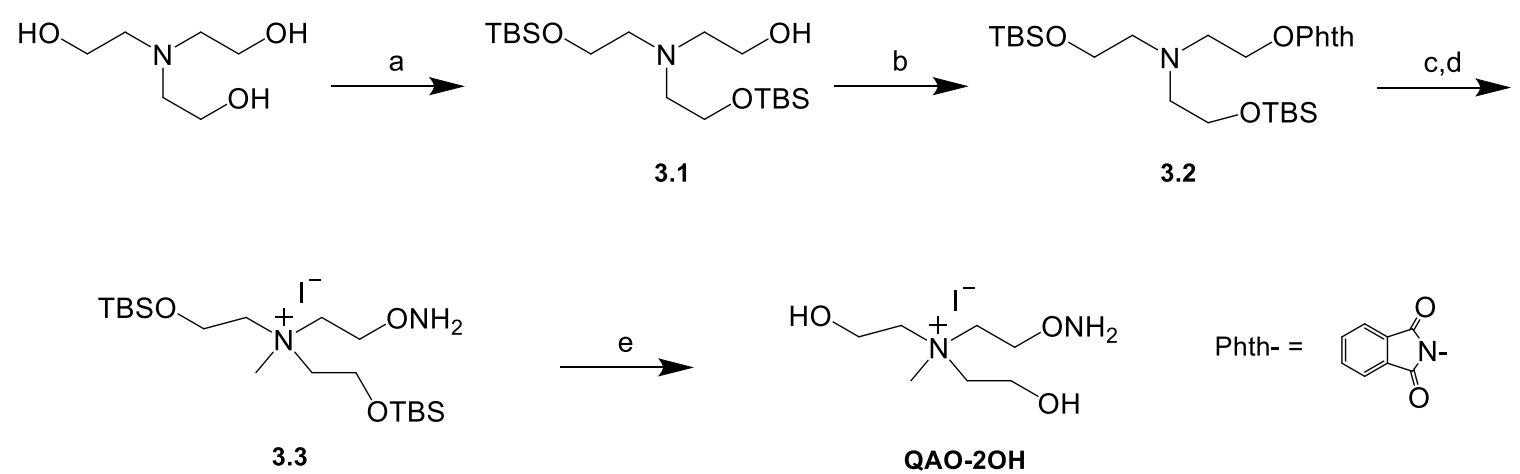

Scheme 3.2. Synthesis of QAO-2OH. Reagents and conditions: (a) TBSCl, $\mathrm{Et}_{3} \mathrm{~N}, \mathrm{CH}_{2} \mathrm{Cl}_{2}, 0^{\circ} \mathrm{C}$-rt, 8h, 13\%; (b) $\mathrm{N}$-hydroxyphthalimide, $\mathrm{PPh}_{3}$, DIAD, THF, $0{ }^{\circ} \mathrm{C}-\mathrm{rt}, 12 \mathrm{~h}, 81 \%$; (c) $\mathrm{CH}_{3} \mathrm{l}, \mathrm{CH}_{2} \mathrm{Cl}_{2}, 50{ }^{\circ} \mathrm{C}$, $6 \mathrm{~h}$ (d) $\mathrm{H}_{2} \mathrm{NNH}_{2}$ monohydrate, EtOH, rt, $12 \mathrm{~h}, 91 \%$ (2 steps); (e) TBAF (1.1 eq), THF, $\mathrm{CH}_{2} \mathrm{Cl}_{2}, 0{ }^{\circ} \mathrm{C}-\mathrm{rt}, 1.5 \mathrm{~h}$, $73 \%$ 


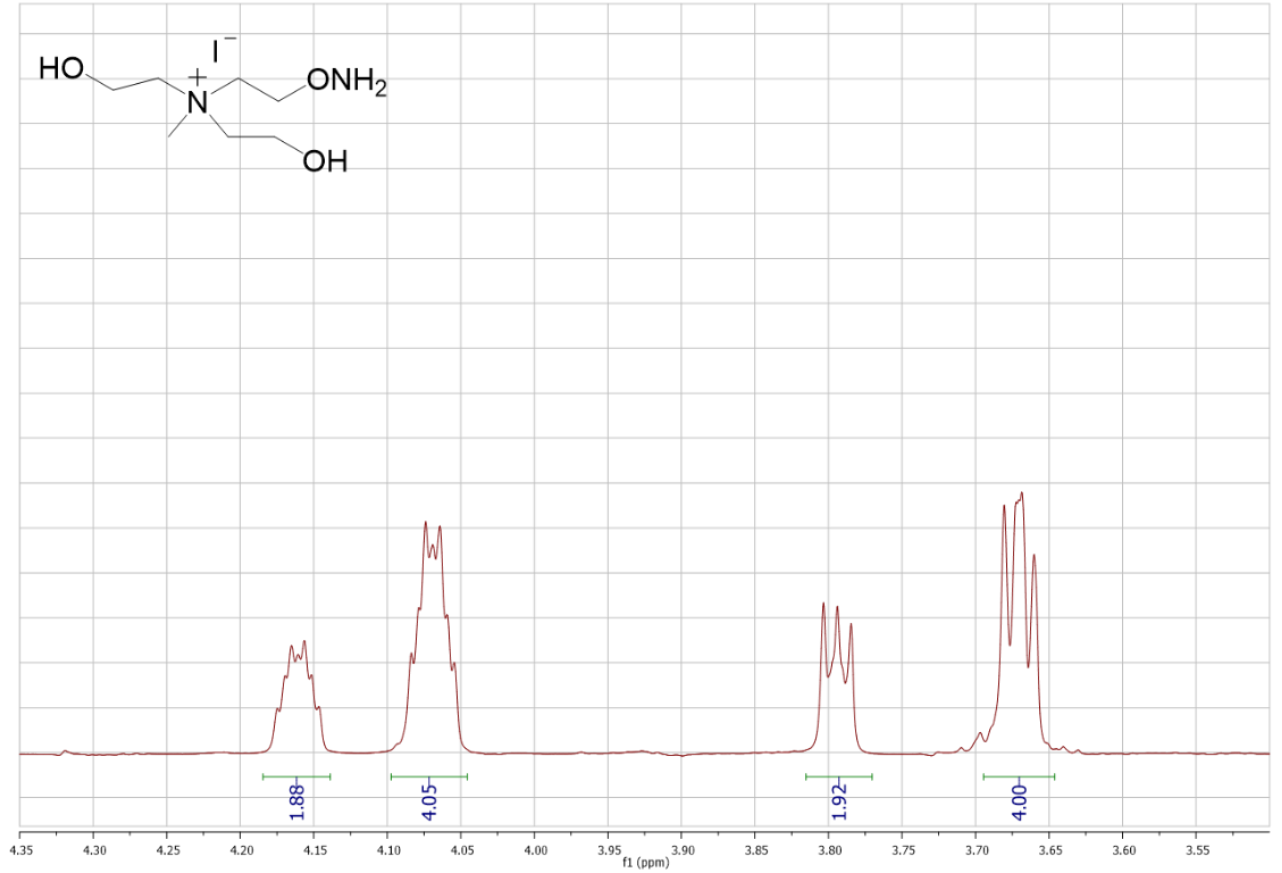

Figure 3.8. ${ }^{1} \mathrm{H}$ NMR expansion of methylene region of $\mathrm{QAO}-2 \mathrm{OH}$ in $\mathrm{D}_{2} \mathrm{O}$

Using a stepwise approach, the three QAOs were first coated onto the NPs followed by addition of FITC-CHO. The resultant oxime ether adducts, FITC-I, II, and III, are pictured in Figure 3.9. As a negative control, FITC-CHO was added to bare NPs as well. The coated NPs generated by this method were then magnetically separated, washed to remove loosely bound fluorophore, and exposed to AMF (201.4A, $203 \mathrm{kHz}, 30 \mathrm{~min})$ to induce $\mathrm{QAO} \bullet \mathrm{FITC}$ conjugate release. After magnetic separation, the supernatant solutions were collected, and fluorophore release was detected by fluorescence HPLC. Prior to this, excitation-emission spectra were acquired on the FITC adducts to verify that QAO linkers did not significantly alter the fluorescent properties of the probe: deviations in excitation maxima varied by less than the excitation beamwidth of the fluorescence detector, and the emission maxima were unaffected by the structural modifications. 


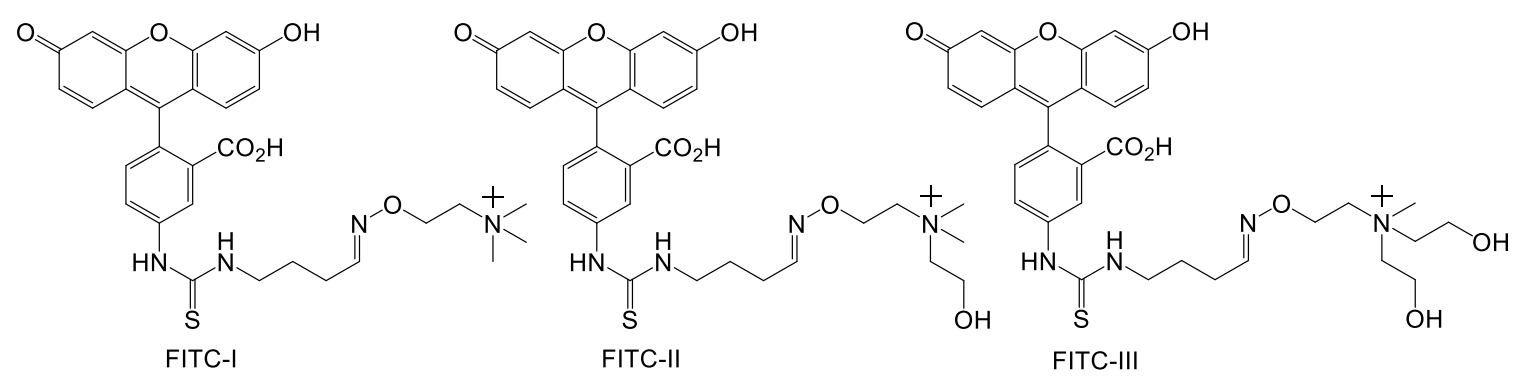

Figure 3.9. Cationic aminooxy-based oxime ether conjugates of FITC-CHO

The results of this initial screen revealed that the negative control showed negligible fluorescence after AMF exposure; thus, no retention of FITC-CHO on NPs in the absence of an aminooxy linker. However, under identical conditions, the NP•FITC-II and NP•FITC-III formulations showed an increase in supernatant fluorescence after AMF exposure indicating release of the conjugates in response to the external stimulus (Figure 3.10). The FITC-I adduct peak, however, could not be resolved from unreacted FITC-CHO in the HPLC run. These results suggested that the hydroxyl motif was important for retention of drug surrogates prior to AMF induction.

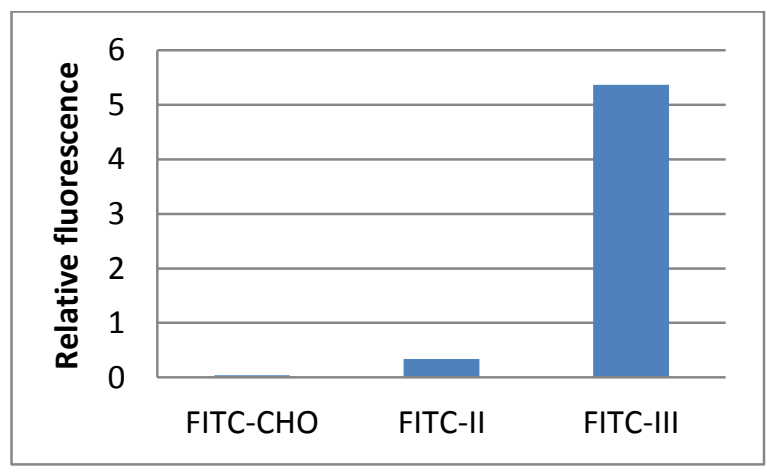

Figure 3.10. Fluorescence intensity of sampled solutions of three formulations of coated NPs 


\subsubsection{Doxorubicin oxime ether analogs}

The model fluorescence study of FITC analogs had supported the idea that AMF could be used to free noncovalently bound species from the surface of NPs. Consequently, we set out to prepare a NP platform with an attached anticancer drug, doxorubicin (Dox, Figure 3.11). As mentioned in the introductory chapter, Dox is a potent chemotherapeutic agent that, in the absence of a targeting agent or nanocarrier, exhibits nonspecific toxicity. The $\mathrm{C}-13$ carbonyl is a convenient reactive partner for a QAO-based aminooxy linker. Thus, we aimed to demonstrate in an in vitro model how iron oxide NPs could be used to deliver modified doxorubicin to cancer cells in response to AMF induction. To simplify the strategy, we altered our methodology to a direct rather than stepwise loading route; we chose for the Dox studies to first generate, purify, and characterize Dox-QAO adducts and then attach the adducts directly onto NPs. Two Dox analogs were prepared using $\mathrm{QAO}-\mathrm{OH}$ and $\mathrm{QAO}-2 \mathrm{OH}$ (Figure 3.6). Since chloride salts were found to bind more effectively than iodide salts in the fundamental study of hydroxylated QAOs, the QAO-Dox conjugates were converted to chloride salts for these studies. The Dox analogs Dox-OH and Dox-2OH (Figure 3.11) were characterized by NMR and high resolution mass spectrometry (Figure 3.12 ) to confirm the formation of the desired oxime ether conjugates.

Upon loading the Dox analogs onto NPs by stirring in DMSO, a color change in the solution from red (the color of Dox $\cdot \mathrm{HCl}$ ) to purple was noted. This may be the result of the formation of an $\mathrm{Fe}^{2+112}$ or $\mathrm{Fe}^{3+}$ complex ${ }^{113}$ as these species have been documented to 
occur and are associated with purple colors. After rinsing to separate unbound Dox analogs, TGA indicated 7.51 weight percent organic material for both species.

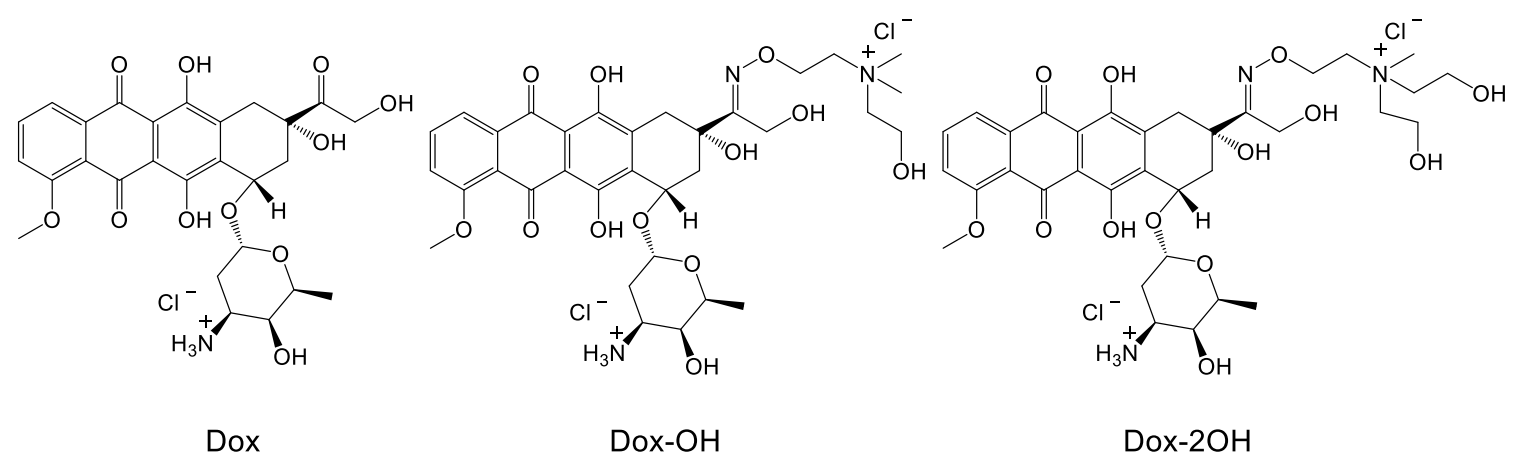

Figure 3.11. Unmodified doxorubicin hydrochloride (Dox) and mono- or dihydroxylated oxime ether conjugates

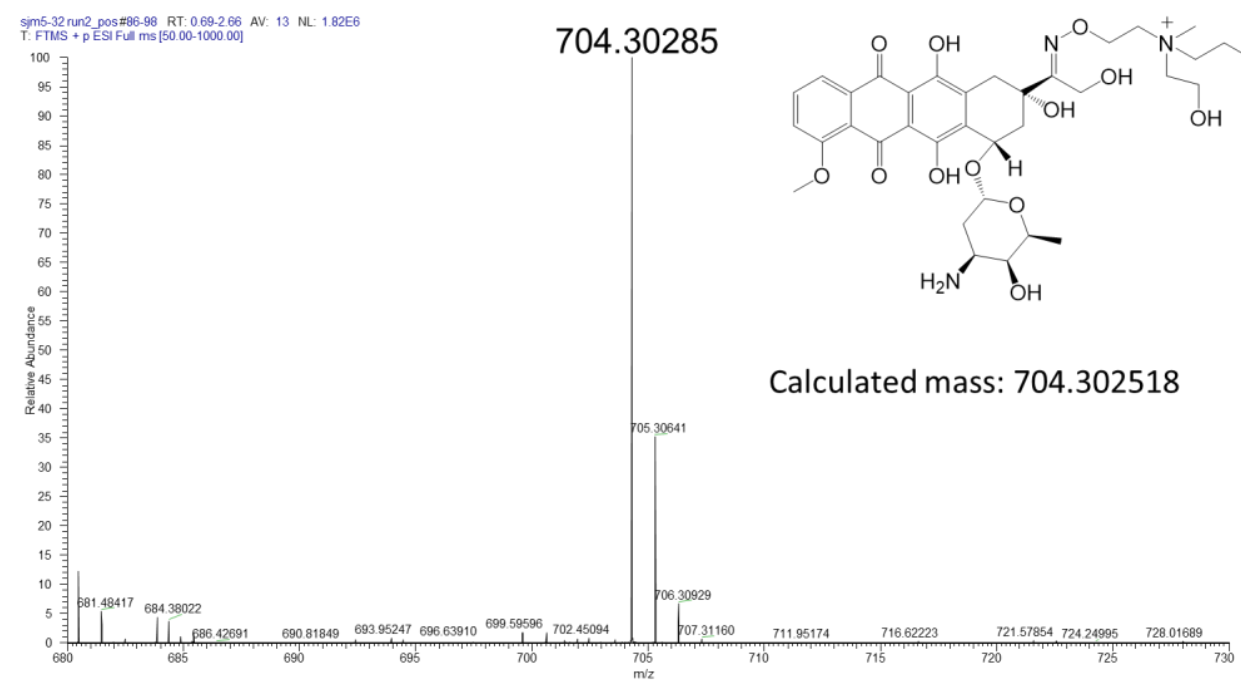

Figure 3.12. FTMS spectrum of Dox-2OH showing the calculated exact mass and the observed peak

\subsubsection{AMF release profiles}

AMF induction of magnetic fluids in living tissue has limitations; nonspecific tissue heating can lead to tissue necrosis. Pulsed AMF, however, has been employed in mouse 
models to avoid this problem. ${ }^{114}$ We looked at the release profiles of Dox-OH and Dox$2 \mathrm{OH}$ during pulsed AMF exposure over one hour. In this study, an aqueous solution of Dox analog-coated NPs received AMF pulses (203 kHz, $350 \mathrm{~A}, \mathrm{rt}$ ) at five minute intervals with intervening five minute incubations at $30{ }^{\circ} \mathrm{C}$. The NPs were then magnetically sedimented and the solution phase absorbance was measured by UV-Vis spectroscopy at $480 \mathrm{~nm}$ (the maximum absorbance for Dox) to measure drug-analog release. The responsiveness of the Dox analogs to AMF induction can be seen in the time course in Figure 3.13 in which the yellow bars indicate AMF "on" time. Although Dox-OH exhibited greater release overall, Dox-2OH release was more closely associated with AMF stimulation; absorbance for Dox-OH rose steadily during AMF pulses as well as during intervening incubation, whereas Dox-2OH showed an increase in absorbance primarily for AMF on times, and flat absorbance during the intervening incubation periods. This increased AMF responsiveness for Dox-2OH may be attributable to a more proximal interaction with the NP surface (facilitating AMF-induced energy transfer), presumably due to the presence of an additional hydrogen bond donor. By comparison with a calibration curve, the 60 minute endpoint corresponded to $15 \mu \mathrm{M}$ Dox-OH and $11 \mu \mathrm{M}$ Dox-2OH. Comparison with TGA data suggested this represented only 4.5 or $5.9 \%$ of payload release for Dox-OH and Dox-2OH respectively, however absorbance declined after further incubation at $30^{\circ} \mathrm{C}$ indicating reattachment of the linker to the NP (likely an equilibrium phenomenon); additionally, in our experience TGA can overestimate loading. The better test for release, we reasoned, would be a non-equilibrium system, one in 
which released Dox analog could be removed from proximity to the NP before readsorbance could occur, specifically, a living system.

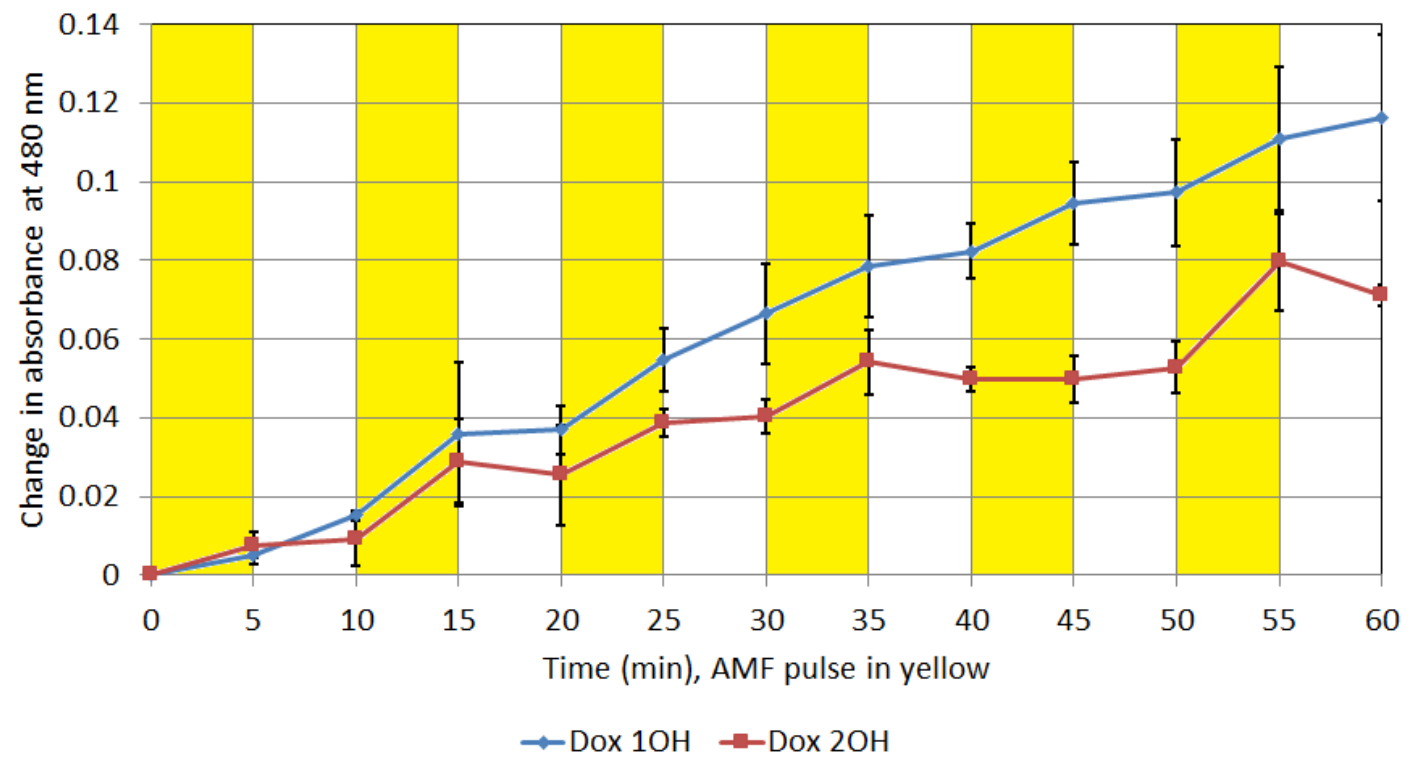

Figure 3.13. UV-Vis detection of Dox analogs released from Dox-NPs by pulsed AMF (203 kHz, $350 \mathrm{~A}, \mathrm{rt}$ ) as indicated by absorbance at $480 \mathrm{~nm}$ of the solution after magnetic separation of the particles. Error bars represent +/- 1 standard deviation for triplicate samples.

\subsubsection{In vitro studies}

A pilot study conducted by my colleague, Dr. Souvik Biswas, on MCF-7 breast cancer cells had generated promising results using NPs conjugated to the iodo form of Dox-OH. ${ }^{99}$ The key findings of his study were an initial demonstration of low toxicity for bare NPs, and high toxicity for NP-Dox-OH formulations upon AMF exposure. Most exciting was the finding that the drug analog displayed very low toxicity in its bound state, but high toxicity upon release, effectively functioning as an inactive pro-drug until external triggering with AMF-a promising scenario. We hoped that the new analogs, 
with chloride counterions and, in the case of Dox-2OH, an additional hydrogen bond donor, could surpass the efficacy of the first generation analog. We also hoped to overcome a challenge encountered with the first generation Dox-OH of premature drug release upon incubation at body temperature ( $\approx 14 \%$ payload loss).

To test the NP-Dox formulations in vitro, MCF-7 breast cancer cells were cultured and plated into either $35 \mathrm{~mm}$ dishes or a 96 well plate modified to fit within the AMF coil. NP-Dox formulations were typically administered by suspending the NPs in PBS and adding the solution to cells in medium. Half of the cells would then be exposed to AMF (350A, $203 \mathrm{kHz}, 3 \mathrm{~min}$ on, 3 min off pulses, $30 \mathrm{~min}$ total time) by placing the culture dish within the coil of the AMF generator. During pulsed AMF application, cells were incubated at $37{ }^{\circ} \mathrm{C}$ between pulses. Cells would then be incubated with treatments for 24 hours after which toxicity would be assayed by trypan blue exclusion and counting of living cells using a hemocytometer.

The toxicity studies presented a number of challenges. As the synthesis and loading of NPs involved a great deal of processing, reproducibility in the toxicity measurements was poor. Small differences in loading concentrations or post-loading washes of the NPs could produce particles with differing amounts of bound drug. Formulations with a lower coating of Dox were more prone to aggregate in PBS buffer resulting in uneven application to cells. As a result, the toxicity studies were highly variable. In a representative toxicity study (Figure 3.14) some effects were consistent between the first generation drug trial and the latter one. For example, AMF alone did not produce significant cell death, nor did bare NPs induced by AMF cause cell death. In 
addition, the NP-Dox-OH formulation did demonstrate a modest degree of toxicity exclusively when AMF was applied. Unfortunately, the NP-Dox-2OH formulation gave an undesirable result showing minor toxicity with or without AMF.

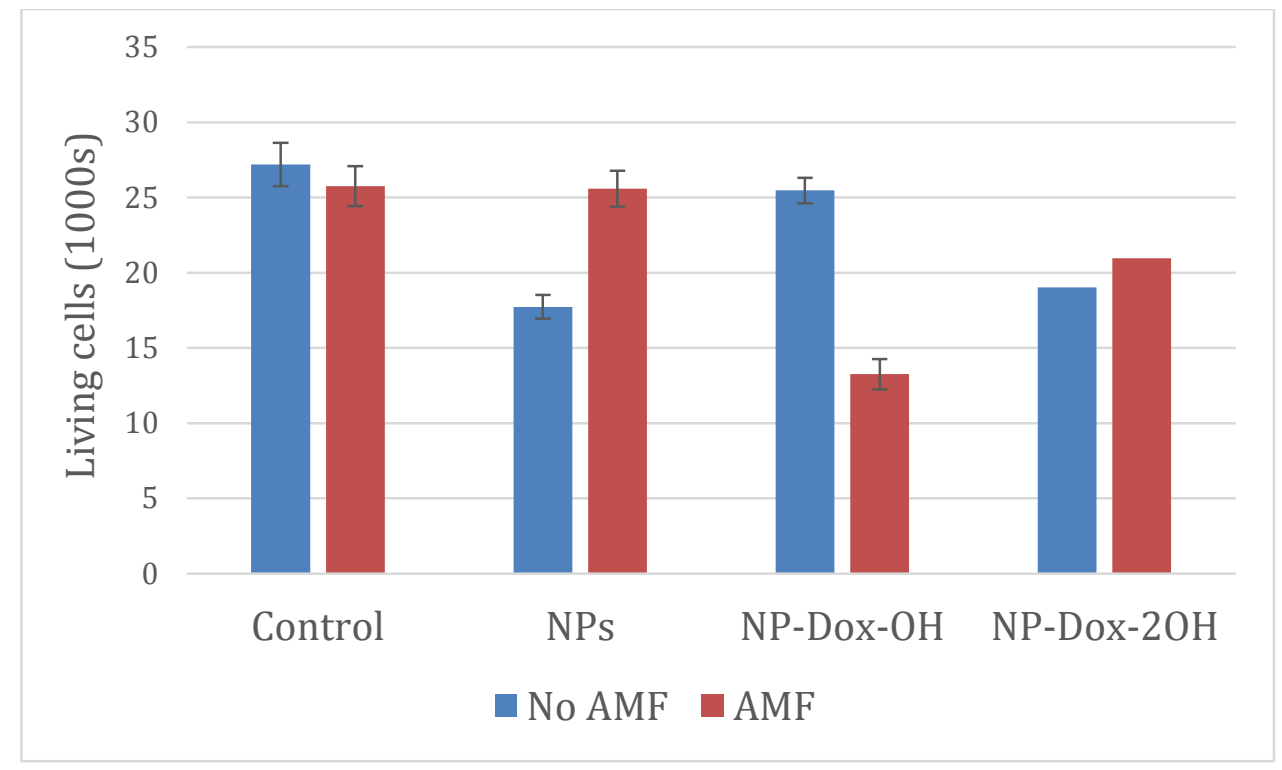

Figure 3.14. Toxicity of NP-Dox formulations and controls on MCF-7 breast cancer cells for cells grown in a 96 well plate to $40 \%$ confluence and treated with $12.3 \mu \mathrm{g} /$ well NP-Dox formulations. Error bars reflect standard error over biological triplicates. NP-Dox-2OH data reflect the average of biological duplicates.

Reproducibility in trials of a NP-mediated drug carrier is crucial for establishing the practical utility of the system. And given the multi-step preparations involved with NP formulations, the components of the system must be robust with predictable interactions. Since our modifications to Dox in this approach led to disappointing results in regard to toxicity, we began to consider modifications to the NP side of the drugnanocarrier interface - how could the surface of the iron oxide NP be altered to actuate the desired drug delivery strategy? 


\subsection{Sulfur-modified NPs}

\subsubsection{Dextran sulfate NPs}

A quaternary ammonium cation is a large, diffuse positive charge. We reasoned that a size-matched large anion on the NP surface could elicit a stronger electrostatic interaction with a QAO than the $\mathrm{Fe}-\mathrm{O}^{-} / \mathrm{OH}^{-}$of the bare particle. ${ }^{115}$ In our first effort to modify the NPs, we elected to change the anion at the $\mathrm{Fe}_{3} \mathrm{O}_{4}$ surface to a sulfate. We selected dextran sulfate as a coating agent to modify our NPs because dextran, a sugar polymer, was already a common coating reagent for NPs.

After coating NPs with dextran sulfate, QAO- $2 \mathrm{OH}$ conjugated to an anthracene fluorophore (Figure 3.15) was added. The release properties under AMF of this chromophore were then measured by UV-Vis. As is shown in Figure 3.16, unfortunately the formulation exhibited slow leakage of the anthracene analog both in the presence and absence of AMF exposure.

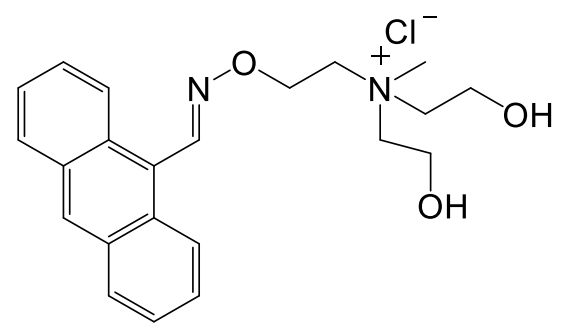

Figure 3.15. QAO-2OH anthracene derivative 


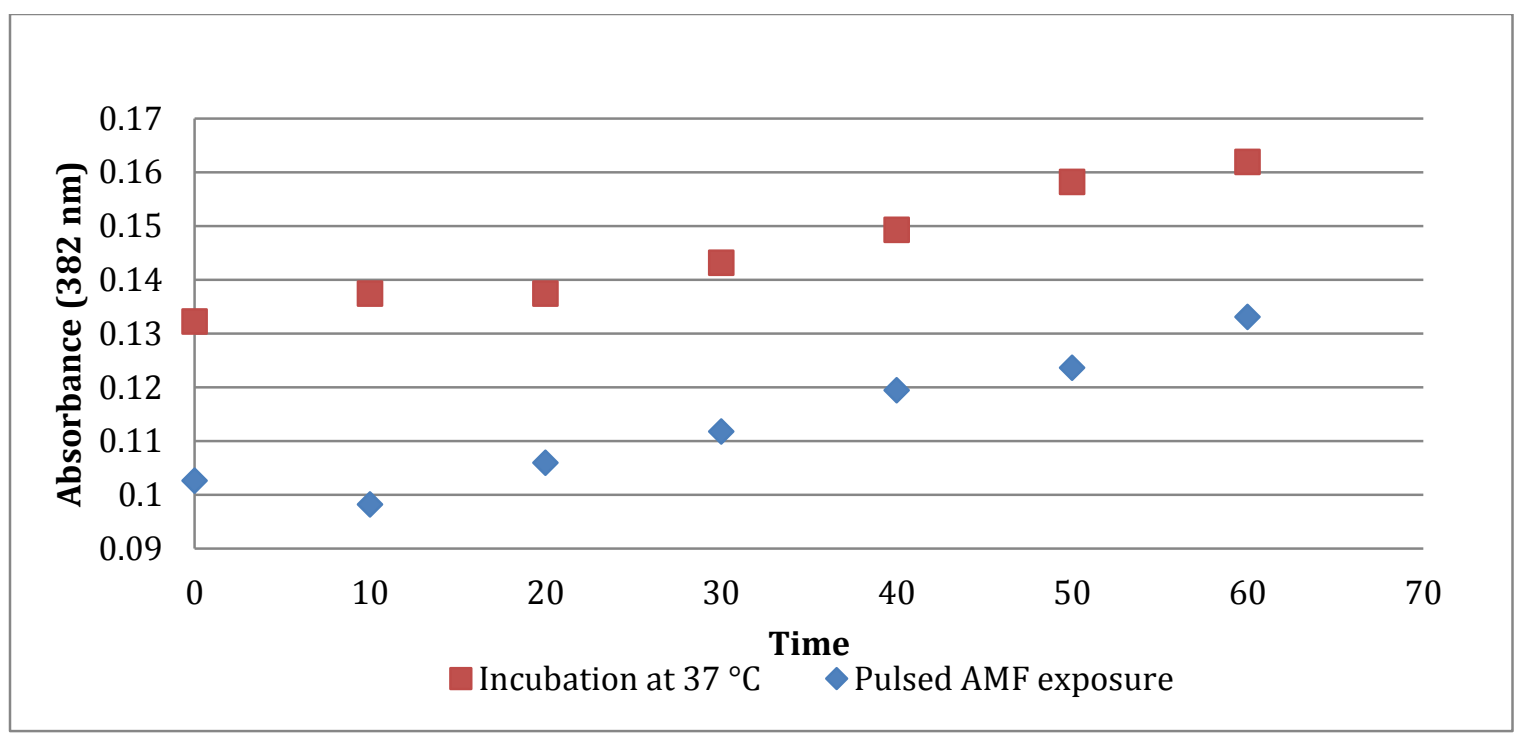

Figure 3.16. AMF-mediated release of anthracene derivative (rt) versus incubation at $37^{\circ} \mathrm{C}$

\subsection{2. $\mathrm{CS}_{2}$-modified NPs}

The next strategy we examined for modifying the NPs was to incorporate xanthate functionality $\left(\mathrm{ROCS}_{2}{ }^{-} \mathrm{M}^{+}\right)$at the iron oxide surface with an eventual aim to pair the constituent dithiocarboxylate anion with a QAO. Xanthates are typically prepared from reaction of an alcohol with carbon disulfide in the presence of base, but we wondered if reaction of iron oxyanions with $\mathrm{CS}_{2}$ might generate an analogous ferric xanthate (Figure 3.17). Some precedent for this concept is found in the adsorption of carbon disulfide and carbonyl sulfide $(\mathrm{O}=\mathrm{C}=\mathrm{S})$ onto iron oxide. ${ }^{116-117}$ In fact, hydrated iron oxides are employed industrially as scrubbers for volatile sulfurous compounds generated during coke fuel production. ${ }^{118}$ Though these triatomic species are known to bind to iron oxide, they have not been applied to NP surface modifications as potential functional organic coatings. 


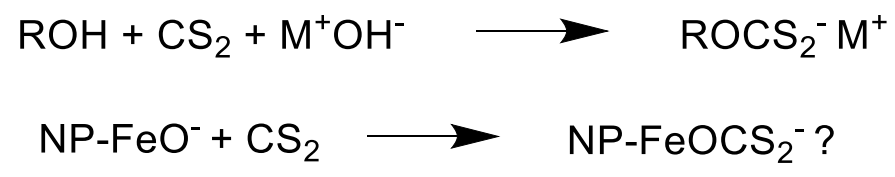

Figure 3.17. Traditional xanthate preparation and ferric xanthate variant.

To ascertain what functional transformations could be made on the surface of the NP, we used the strategies summarized in Figure 3.18. Carbon disulfide was reacted with bare NPs $\mathbf{1}$ to produce product $\mathbf{2}$. The zeta potential of $\mathbf{2}$ remained negative (bare NPs: 35.9; $\mathrm{CS}_{2}$-coated: -29.3), indicating either no reaction or the formation of a sulfanion. There were indications that some type of reaction had taken place because the color and texture of the NPs changed upon coating, and off-gassing was noted after $\mathrm{CS}_{2}$ addition and workup (rinsing and drying the NPs). Further, an IR of the product in comparison with bare NPs 1 showed the emergence of two new peaks in the fingerprint region at 1231 and $1212 \mathrm{~cm}^{-1}$. 


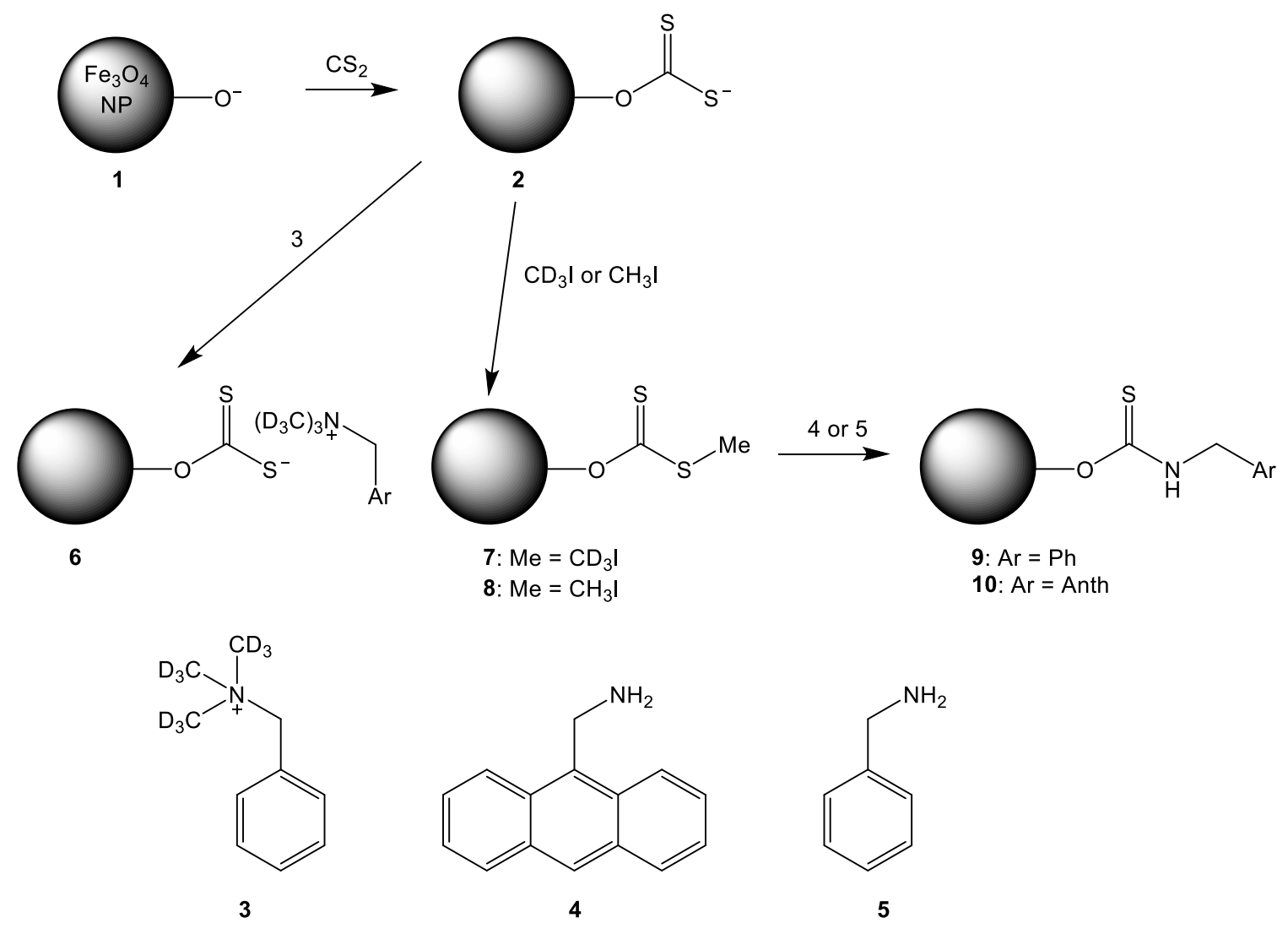

Figure 3.18. Strategies for incorporating organics onto sulfur-modified NPs.

The $\mathrm{CS}_{2}$-modified NPs 2, were then reacted with either deuterated iodomethane, to cap the sulfanion with a methyl group, or they were mixed with deuterated ammonium salt 3 to test their potential for electrostatically binding substrates. Deuterated reagents were selected in the hopes that attenuated total reflectance IR (ATR-IR) of the NPs on a germanium crystal could detect the C-D stretching vibrations of the products, as this vibration occurs in an otherwise sparse region of the IR spectrum $\left(2075 \mathrm{~cm}^{-1}\right)$.

C-D stretches for compounds 6 and 7 were not observed, however surface changes were evident using other characterization techniques. Figure 3.19 shows the results of elemental analysis (EA) that gives the weight percent of carbon, hydrogen, and 
sulfur for NPs $\mathbf{1}, \mathbf{2}, \mathbf{8}$, and a control prepared by addition of iodomethane to bare NPs $\mathbf{1}$. The values displayed in the graph represent the weight percent of each element divided by its molecular weight to give relative moles of each element. Of course, the bulk of the weight of these NP formulations is iron and oxygen which are not represented in this graph. EA revealed an increase in the sulfur content of NPs $\mathbf{2}$ as compared with bare NPs 1, but surprisingly no increase in the carbon content. EA of 8 showed an increase in carbon content, but not the 3:1 ratio of $\mathrm{H}$ to $\mathrm{C}$ that would be expected for methyl group addition. Meanwhile the sulfur content decreased for compound 8. Most surprisingly, the negative control showed an increase in carbon content implying retention of iodomethane (b.p. $40^{\circ} \mathrm{C}$ ) on bare NPs, despite $24-48$ hours under vacuum.

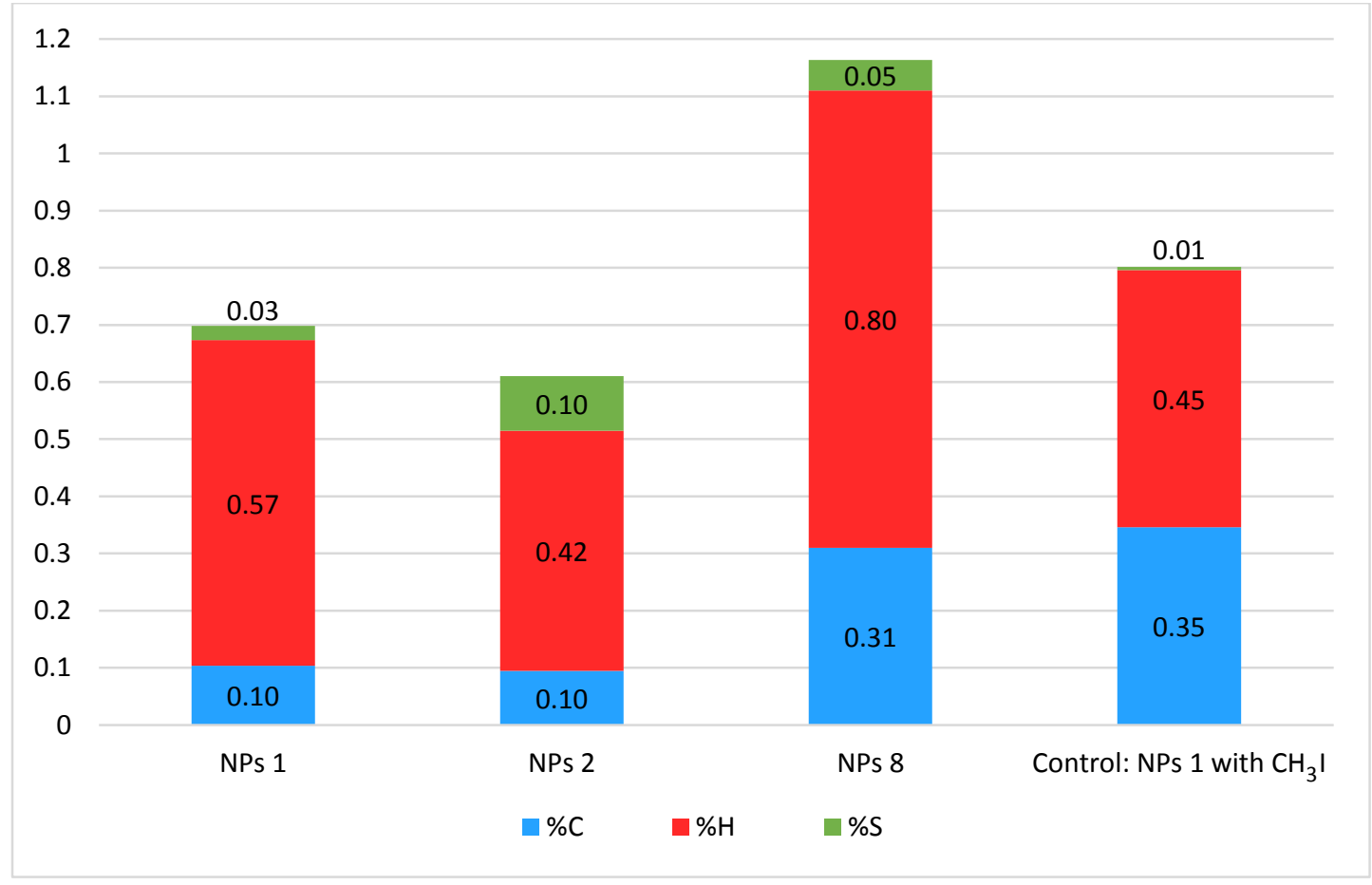

Figure 3.19. Elemental analysis of NPs formulations from Figure 3.18 
TGA also demonstrated high electrostatic binding properties for the $\mathrm{CS}_{2}$-modified NPs. TGA of NPs 6 showed a large amount of bound ammonium salt $\mathbf{3}$ compared to a control of bare NPs 1 with the same salt (Figure 3.20). The weight loss curves were integrated above $200{ }^{\circ} \mathrm{C}$ to exclude weight loss due to adsorbed water from the calculation.

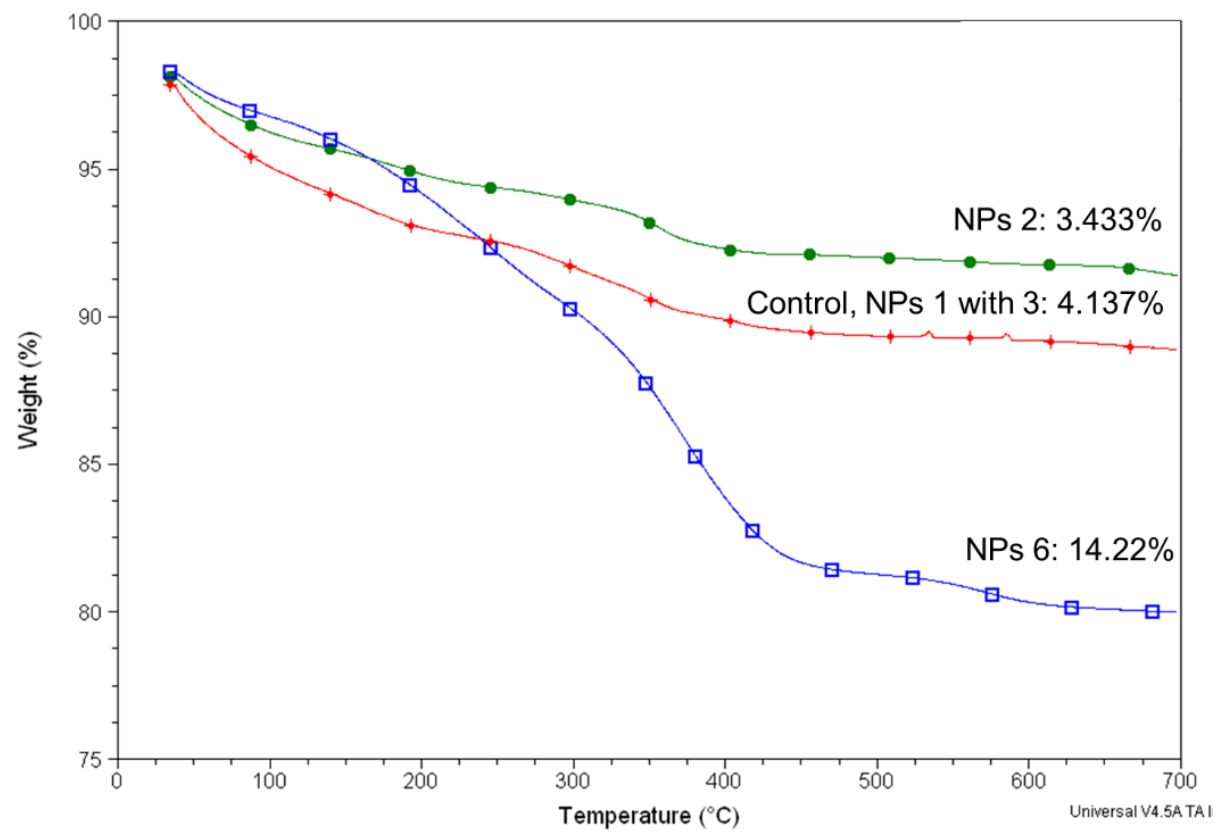

Figure 3.20. TGA curves for select NPs from Figure 3.18

Presuming formation of methyl xanthate $\mathbf{8}$, amines $\mathbf{2}$ and $\mathbf{3}$ were prepared in the hopes of forming more stable thiocarbamates $\left(R O C(=S) N R_{2}\right) \mathbf{9}$ and 10 by displacing methane thiolate. IR of 9 revealed aromatic $\mathrm{C}-\mathrm{C}$ stretching vibrations, $\mathrm{C}-\mathrm{H}$ stretching, and a peak at $1036 \mathrm{~cm}^{-1}$, close to the reference peak for $\mathrm{C}=\mathrm{S}$ of $1050 \mathrm{~cm}^{-1}$. And MALDI-TOFMS of 9 detected a peak at $m / z 108$, the mass of the protonated form of 3 (108.08). Compound 10 showed the greatest change in morphology with NPs turning from brown 
to beige after coating. TGA validated the heavy loading on NPs 10 indicating 28.6 weight percent of bound organic.

We wondered how the $\mathrm{CS}_{2}$-modified NPs would compare to unmodified NPs in affinity for hydroxylated ammonium salts. Using the earlier study as our model, we mixed $\mathrm{CS}_{2}$-modified NPs 2 with the variably hydroxylated ammonium salts from Figure 3.4. As can be seen from Figure 3.21, we did not observe the same trend for greater binding with increased hydroxylation, nor did the counterion have the same effect. Strangely, the chloride salts appeared to displace some of the bound $\mathrm{CS}_{2}$.

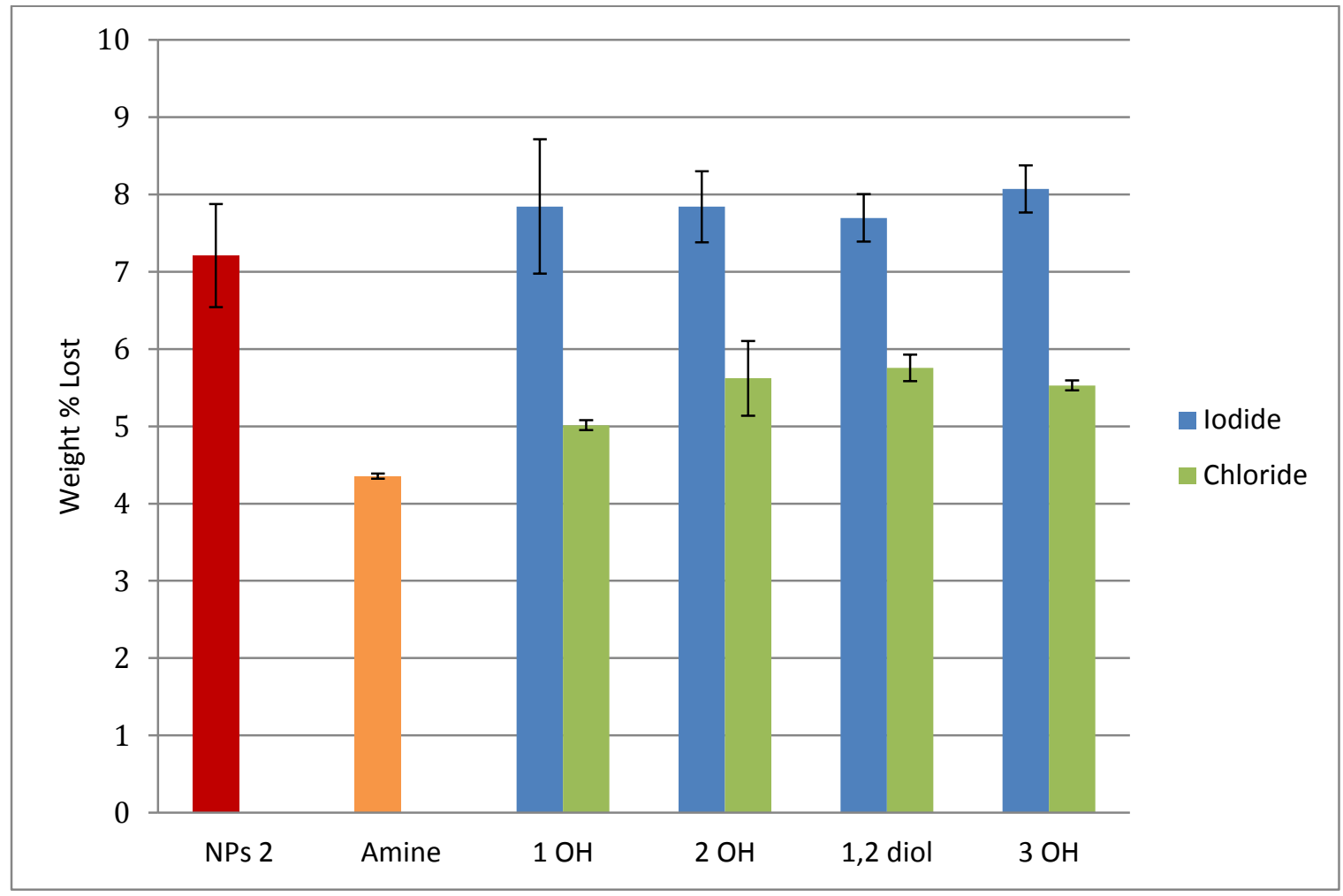

Figure 3.21. Weight \% of bound organics based on TGA of CS2-modified NPs with variably hydroxylated ammonium salts.

Although we were intrigued by the properties of the $\mathrm{CS}_{2}$-modified NPs, there was a lack of clarity as to the exact nature of its interaction with the NP surface. Prior to these 
experiments, we had conceived of the NPs as passive platforms for adsorbing organics, but the unexpected results of the EA in particular indicated a surface reactivity or catalytic activity that we had failed to appreciate. A deeper look into the literature on the interaction of iron oxide with species like $\mathrm{CS}_{2}$ confirmed this reactivity. Wang et al., for example, identified oxidation of $\mathrm{CS}_{2}$ on iron oxide atmospheric particles to carbonyl sulfide (COS). ${ }^{119}$ In fact, this is something we had observed with our own NPs; GC-MS of the headspace during off-gassing of $\mathrm{CS}_{2}$-modified NPs had shown a mass corresponding to COS. And neither is such catalysis exclusive to $\mathrm{CS}_{2}$. Chen and coworkers found oxidation of $\mathrm{COS}$ to $\mathrm{CO}_{2}$ on hematite, another form of iron oxide. The proposed mechanism is given in Figure 3.22. ${ }^{120}$ Dissociative chemisorption of carbon dioxide on iron oxide has also been proposed. ${ }^{121}$ Even room temperature water splitting has been observed on the surface of magnetite. ${ }^{122}$ These phenomena would eventually lead us to a rethinking of attachment of sensitive molecules to NP surfaces. Rendering the surface more inert would become a priority. 

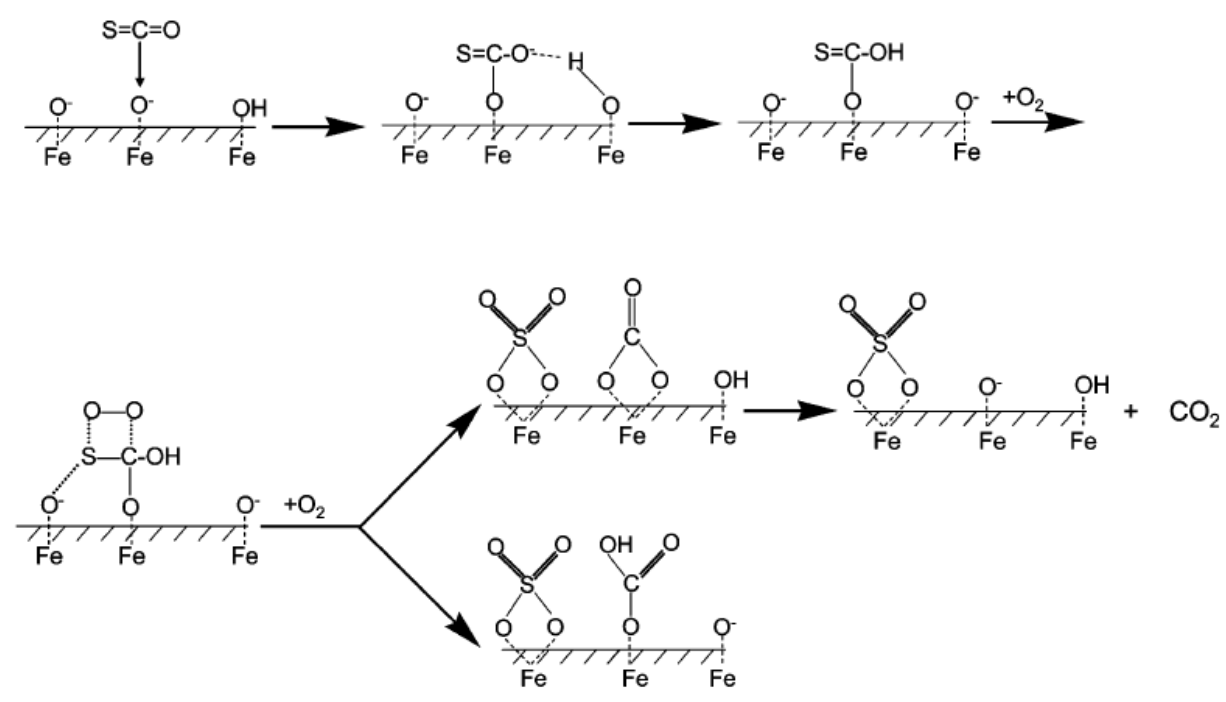

Figure 3.22. Proposed mechanism for oxidation of $\mathrm{COS}$ to $\mathrm{CO} 2$ on hematite. Reprinted with permission from Environ. Sci. Technol. 2007, 41 (18), 6484-6490. Copyright 2007 American Chemical Society.

In our final investigation into sulfur-modified NPs, we turned our attention to an entirely different route for putting sulfur on the surface, one that makes sulfur an intrinsic component of the NPs themselves.

\subsubsection{Greigite NPs}

Iron sulfide, $\mathrm{Fe}_{3} \mathrm{~S}_{4}$, or greigite, shares many properties with its cousin, $\mathrm{Fe}_{3} \mathrm{O}_{4}$. Like magnetite, it has a spinel crystal structure and is magnetic. Greigite NPs can be prepared from an acidified mixture of iron II sulfate and sodium sulfide as described by Chang and colleagues. ${ }^{123}$

To assess whether the presence of sulfur anions as opposed to oxyanions at the particle surface could enhance adsorbance of quaternary amines, akin to the $\mathrm{CS}_{2}$ modified NPs, we prepared greigite NPs and coated them with the QAO-2OH anthracene 
derivative pictured in Figure 3.15. TGA of the isolated product (Figure 3.23) showed that iron sulfide, like iron oxide, could bind ammonium salts.

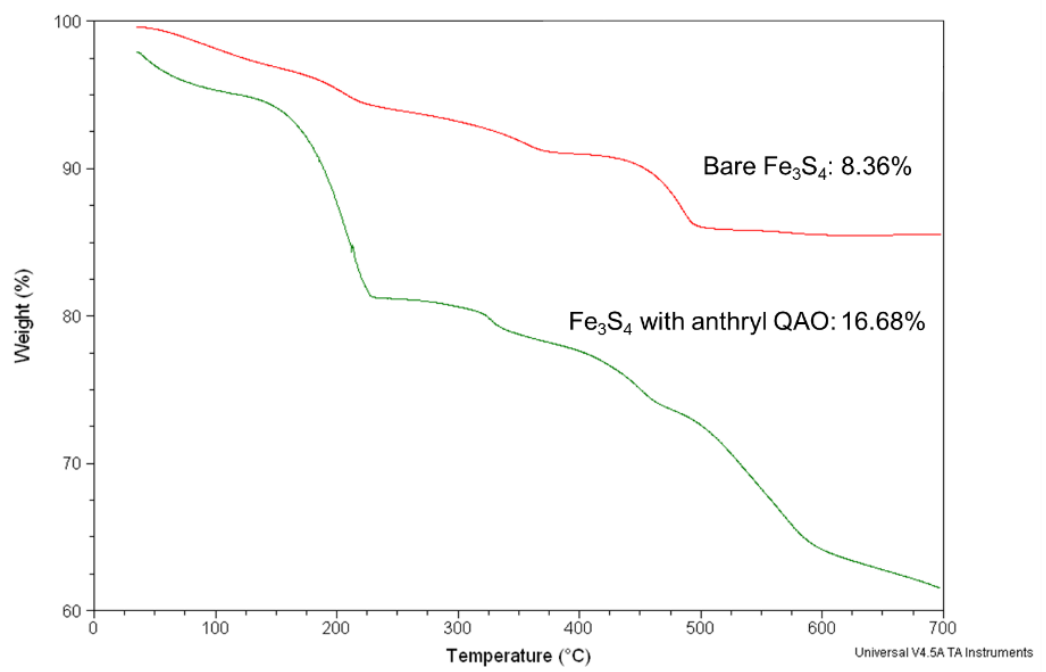

Figure 3.23. TGA showing percent weight lost from bare $\mathrm{Fe}_{3} \mathrm{~S}_{4} \mathrm{NPs}$ or QAO$2 \mathrm{OH}$ anthracene-loaded particles.

AMF release properties of the anthracene adduct from the $\mathrm{Fe}_{3} \mathrm{~S}_{4} \mathrm{NPs}$ were then assessed by UV-Vis at $384 \mathrm{~nm}$, however no increase in solution absorbance relative to incubation at $30{ }^{\circ} \mathrm{C}$ was observed. When the heating properties of $\mathrm{Fe}_{3} \mathrm{~S}_{4} \mathrm{NPs}$ were examined it was discovered that the greigite NPs did not heat significantly upon exposure to $A M F$, likely the culprit for the absence of release.

It was at this point that we decided to retool our strategy for binding organics to NPs. Iron oxide had proven the more efficient material for the sake of AMF heating, so we would return to that architecture, but the surface reactivity would need to be neutralized by coating with a more inert material. As discussed in the next chapter, we would turn to silica coating to serve this purpose. 


\section{CHAPTER 4 CATIONIC MAGNETOLIPOSOMES FOR DRUG DELIVERY}

4.1. Composite NPs: silica on iron oxide

4.2. Cationic magnetoliposomes

4.3. Results and discussion

4.3.1. Cationic oxime ether lipids

4.3.2. Preparation of Dox-AH

4.3.3. $\mathrm{CML}$ formation and stability

4.3.4. Titration of SNP with cationic lipid 1

4.3.5. Estimation of lipid : Dox-AH ratio

4.3.6. Cytotoxicity study

4.3.7. AMF and bilayer disruption

4.4. Gene transfection studies

4.4.1. Binding affinity

4.4.2. Transfection efficiency

4.4.3. Cell viability 
4.4.4. Gene expression

4.5. Conclusions 


\subsection{Composite NPs: silica on iron oxide}

Modifications to the surface of iron oxide NPs are often required to generate stable aqueous colloids and to render the catalytically active ${ }^{119,121}$ surface inert for the sake of ligand/drug stability. Silica coating also allows for functional group interconversion to a variety of other functionalities including alkyne, azide, carboxyl, nitrile, phosphate, and thiol. ${ }^{124}$ To this end, coating NPs with silicon dioxide is commonly employed, particularly for multi-component nanosystems. ${ }^{125}$ Creating a core-shell $\mathrm{Fe}_{3} \mathrm{O}_{4}$ $\mathrm{SiO}_{2}$ structure is desirable because the silica shell enhances the hydrophilicity of the particles and reduces cellular toxicity, ${ }^{126}$ while largely preserving their magnetic properties. ${ }^{127}$ Moreover, as silicon is a metalloid, rather than a metal, the composite structure has a less reactive surface than iron oxide.

The surface of silica-coated NPs (SNPs) is inherently negatively charged due to the presence of silanol groups, but positively charged particles may be more desirable for oncological applications considering the favorable pairing of cationic particles with the negative exterior of many cells, including cancer cells. ${ }^{128-129}$ Further, a self-assembly process for formation of a therapeutic nanosystem is desirable since ultimate broad application would depend upon its ease of preparation. 


\subsection{Cationic magnetoliposomes}

Dual-chain lipids, typically zwitterionic phospholipids, have been used to coat NPs and have been shown to self-assemble into bilayers surrounding anionic NPs forming what are termed classical magnetoliposomes. ${ }^{130}$ In that the lipid is directly bound to the particle surface, these constructs differ from extruded magnetoliposomes that are composed of NPs entrapped within the aqueous core of a lipid vesicle. ${ }^{131}$ Direct attachment of lipids at a NP surface offers the advantage of size control. Namely, the size of the magnetopliposome is dictated by the dimensions of the NP, a tunable property. Thus classical magnetoliposomes are usually smaller $(<100 \mathrm{~nm})$ than their extruded counterparts. ${ }^{131}$ In addition, the preparation of extruded liposomes generally requires more involved techniques, such as sonication or freeze-thaw cycling. Positively charged magnetoliposomes, termed "cationic magnetoliposomes" (CMLs), are typically formed from a mixture of zwitterionic phospholipids, cationic aminium lipids, and neutral lipids. Marcel De Cuyper and Stefaan Soenen have studied such cationic magnetoliposomes ${ }^{132}$ extensively, elucidating their cellular interactions, toxicity, and cell-labeling potential. ${ }^{133-}$ 140

Cationic lipids containing a quaternary ammonium headgroup, and thus a permanent positive charge, are known in the context of gene transfection to promote rapid cellular uptake when complexed with nucleic acids in a "lipoplex". Their high charge density encourages association with cell membranes and facilitates endocytosis. ${ }^{141-142}$ This capacity has also been exploited in concert with NPs in the practice of magnetofection where cationic lipid-coated NPs have been explored as magnetic gene 
vectors. ${ }^{141,143-144}$ As the surface of SNPs, like iron oxide, is anionic, we envisioned constructing a CML that could auto-assemble by simple mixing of a custom quaternary ammonium-based lipid and SNPs.

We aimed to prepare a self-assembling CML-SNP tailored for biological applications with suitable size and charge character for efficient cellular internalization. To demonstrate the potential of the system as a nanocarrier, we prepared an amphiphilic derivative of Doxorubicin (Dox) for incorporation into the CML bilayer. We envisioned that mixing of SNPs, our custom cationic lipid, and the Dox analog would produce a CMLSNP drug carrier (Figure 4.1).

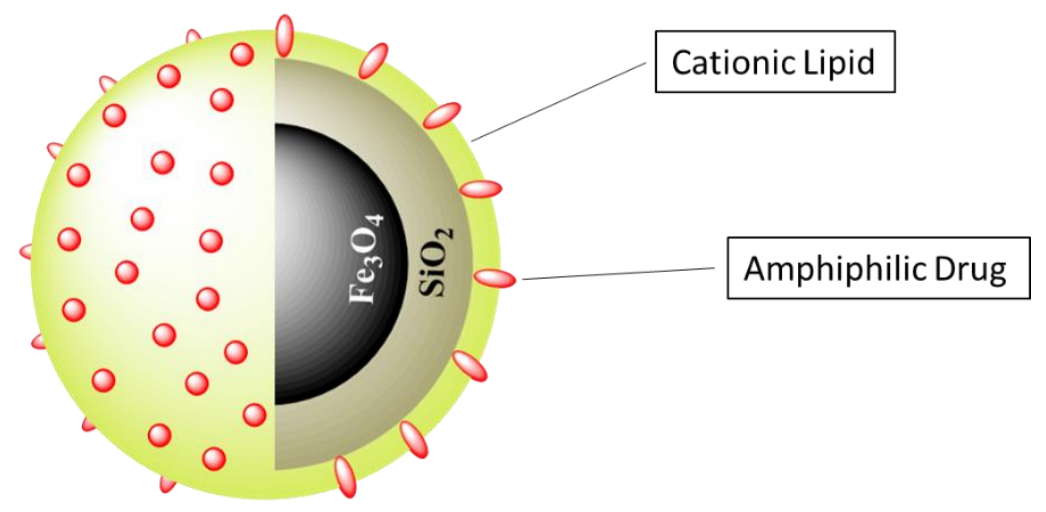

Figure 4.1. Cationic magnetoliposome with a core-shell iron oxide-silica NP surrounded by a cationic lipid bilayer and a bilayer-embedded amphiphilic drug

\subsection{Results and Discussion}

\subsubsection{Cationic oxime ether lipids}

Traditionally, lipids are constructed with ester linkages that tether hydrophobic and polar domains. In conceiving of a lipid to coat SNPs for Dox delivery applications, we turned to oximation for lipid construction. By mixing an aminooxy headgroup with 
aldehyde tails of the desired chain lengths and unsaturation, a wide variety of oxime ether lipids can be prepared. ${ }^{141,145}$

The panel of oxime ether-based cationic lipids we synthesized for loading onto SNPs were designed with quaternary ammonium headgroups as the permanent positive charge ensures that $\mathrm{pH}$ changes in the particle environment will not alter the electronic character of the lipid. Building on our work with hydroxylated $\mathrm{QAOs}$ and $\mathrm{Fe}_{3} \mathrm{O}_{4} \mathrm{NPs}$, we also added hydroxyl functionality to the lipid headgroups to encourage association with the hydrophilic surface of the SNPs. Hydroxyl groups have also been shown to enhance cell membrane interaction. ${ }^{146}$

Three lipids were prepared (Scheme 4.1 and Figure 4.2) with varying chain lengths (C12, C14, and C18) by reaction of aminooxy 4.1 with 2 equivalents of aldehydefunctionalized alkyl chains. The bis-oxime ether adducts (4.2-4.4) were formed as mixtures of $E, E$, and $E, Z$ isomers and were not typically isolated as separate diastereomers. In Figure 4.3 the ${ }^{1} \mathrm{H}$ NMR triplets for the oximyl protons in $E$ and $Z$ configurations are shown for a representative lipid. The dissymmetry provided by incorporation of the $(E, Z)$-containing lipid fraction likely will contribute to the formation of more fluid lipid bilayers as it prevents tight lipid packing. 


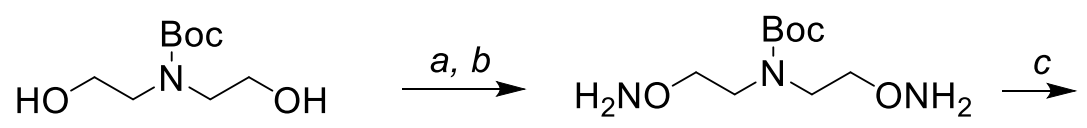

\section{1}<smiles>[R]C=NOCCN(CCO/C=N\C[R])C(=O)O</smiles><smiles>[R]C=COCCN(CCON=C[R])CC(O)CO</smiles>

4.2: $\mathrm{R}=\left(\mathrm{CH}_{2}\right)_{10} \mathrm{CH}_{3}$

4.3: $\mathrm{R}=\left(\mathrm{CH}_{2}\right)_{12} \mathrm{CH}_{3}$

4.5: $\mathrm{R}=\left(\mathrm{CH}_{2}\right)_{10} \mathrm{CH}_{3}$

4.4: $\mathrm{R}=(\mathrm{Z})-\left(\mathrm{CH}_{2}\right)_{7} \mathrm{CH}=\mathrm{CH}\left(\mathrm{CH}_{2}\right)_{7} \mathrm{CH}_{3}$

4.6: $\mathrm{R}=\left(\mathrm{CH}_{2}\right)_{12} \mathrm{CH}_{3}$

4.7: $\mathrm{R}=(\mathrm{Z})-\left(\mathrm{CH}_{2}\right)_{7} \mathrm{CH}=\mathrm{CH}\left(\mathrm{CH}_{2}\right)_{7} \mathrm{CH}_{3}$

Scheme 4.1. Conditions: a. $\mathrm{N}$-hydroxyphthalimide, $\mathrm{PPh}_{3}, \mathrm{DIAD}, 0{ }^{\circ} \mathrm{C}$ to rt, 12h; b. $\mathrm{N}_{2} \mathrm{H}_{4} \cdot \mathrm{H}_{2} \mathrm{O}, \mathrm{CH}_{2} \mathrm{Cl}_{2}$, rt, $2.5 \mathrm{~h}$ (16\%, 2 steps); c. corresponding dodecyl, tetradecyl, or oleyl aldehyde, $\mathrm{CH}_{2} \mathrm{Cl}_{2}, \mathrm{rt}$, 10h (47-71\%); d. TFA, $\mathrm{CH}_{2} \mathrm{Cl}_{2}, 0^{\circ} \mathrm{C}$ to rt, 3h (91-100\%); e. glycidol, EtOH, rt, 60h (38-41\%); f. $\mathrm{CH}_{3} \mathrm{l}, \mathrm{CH}_{2} \mathrm{Cl}_{2}$ (sealed tube), $55^{\circ} \mathrm{C}$, 5h (63-64\%).

1:

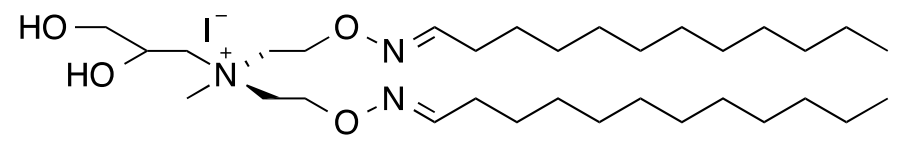

2:

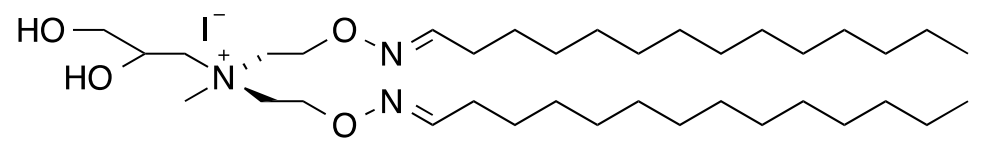

3:

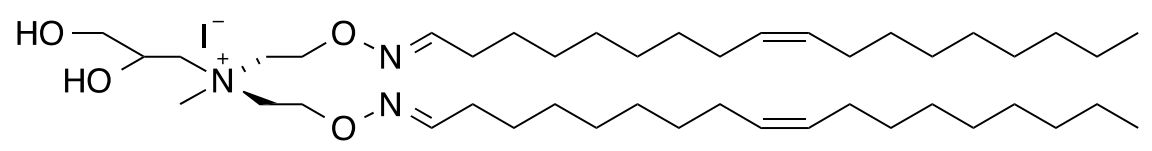

Figure 4.2. Cationic oxime ether lipids 


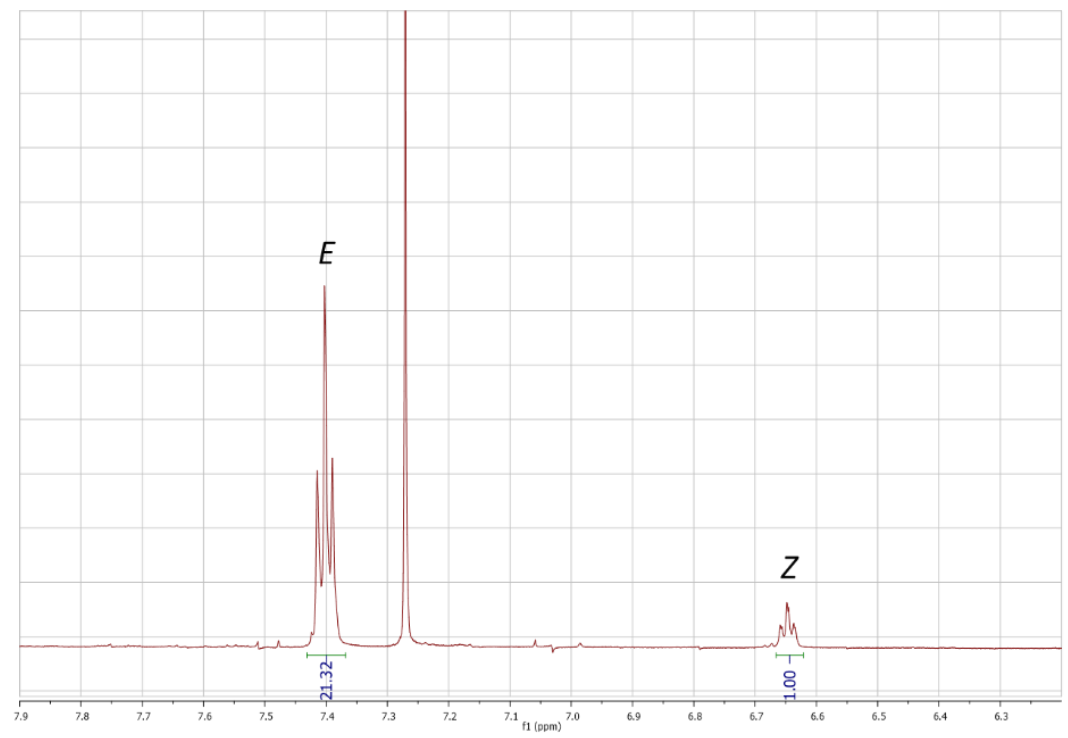

Figure 4.3. ${ }^{1} \mathrm{H}$ NMR expansion showing oximyl proton shifts for $E$ and $Z$ configurations of lipid $\mathbf{2}$

To add hydroxyl functionality to the lipid headgroup we turned to nucleophilic addition of the amines generated from step $d$ of Scheme 4.1 to the C-3 carbon of glycidol (Figure 4.4) as a strategy for installing a 1,2, diol functionality. ${ }^{147}$ Normally the reaction is performed at elevated temperatures, with an excess of amine, or catalysts such as lithium perchlorate. ${ }^{148-149}$ Kuwabe et al., ${ }^{150}$ for example, reported addition of a secondary amine to glycidol after 48 hours refluxing in ethanol. However, a recent report of room temperature reaction of secondary amines with glycidol in water ${ }^{148}$ piqued our interest. As our starting material was not soluble in water, we instead used ethanol. Reaction of bis-oxime ether secondary amines with glycidol in ethanol (rt, 24h) was successful in yielding 1,2 diol substituted tertiary amines. A representative FT-MS spectrum is depicted in Figure 4.5. 

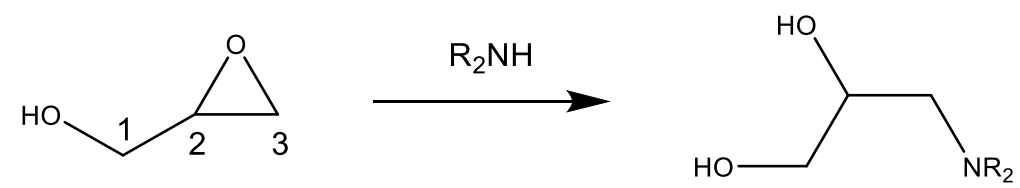

Figure 4.4. Nucleophilic addition of a secondary amine to C-3 of glycidol

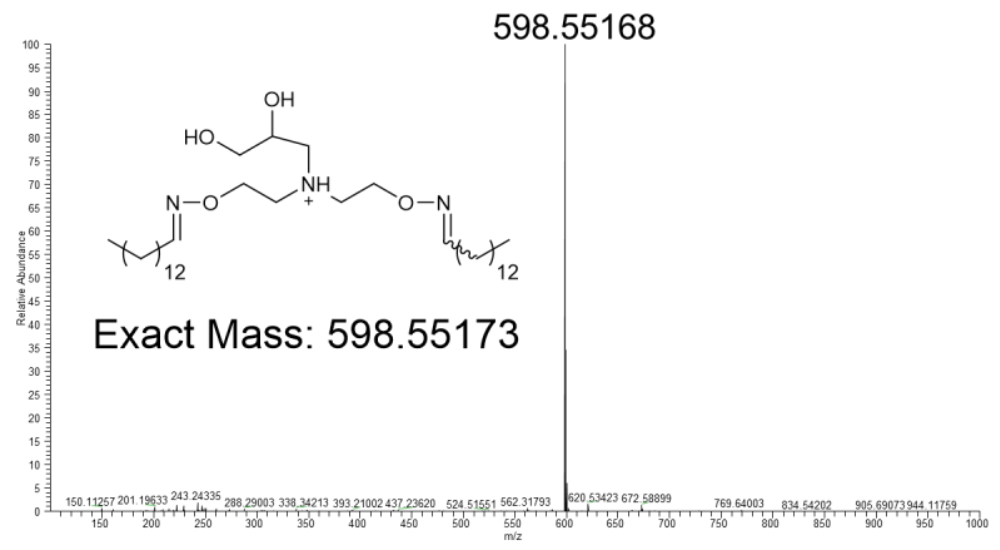

Figure 4.5. Calculated exact mass of $\mathbf{4 . 6}$ and FTMS spectrum of the observed mass

\subsubsection{Preparation of Dox-AH}

Model drug, Dox, can be modified at the C-13 carbonyl group through condensation with a hydrazide, to from an acyl hydrazone. In this way, the lipophilicity of an otherwise highly hydrophilic drug is increased, better suiting it for incorporation into a lipid bilayer.

Several examples of Dox C-13 hydrazones have been documented in the literature. Effenberger et al. ${ }^{151-152}$ prepared Dox hydrazones using $\mathrm{C}_{17}$ and $\mathrm{C}_{18}$ fatty acids and menthyl/bornyl esters. The cytotoxic efficacy of these analogs and mechanism of cell death were investigated in several cancer lines, and in many cases the derivatives were 
more effective than the native form. Particularly interesting was the efficacy against multidrug resistant cells, possibly a consequence of superior diffusion of the analogs through cellular membranes. ${ }^{153}$ Maleimide substituted acyl hydrazones of Dox have also been prepared and demonstrated to be practical derivatives, such as aldoxorubicin, currently in phase II clinical trials. ${ }^{154-155}$ Consequently, to adjust lipophilicity, we prepared a C-13-hydrazone (Scheme 4.2) by reaction of Dox with hexadecanoic acid hydrazide in the presence of an aniline activator ${ }^{156}$ to obtain Dox acyl hydrazone Dox-AH. Hydrazone formation was verified by ${ }^{13} \mathrm{C}$ NMR where the disappearance of the $\mathrm{C}-13$ carbonyl peak at $213.6 \mathrm{ppm}$ was noted along with the appearance of a peak at $152.2 \mathrm{ppm}$ (Figure 4.6). This ppm shift is comparable to that reported for a similar Dox hydrazone. ${ }^{154}$

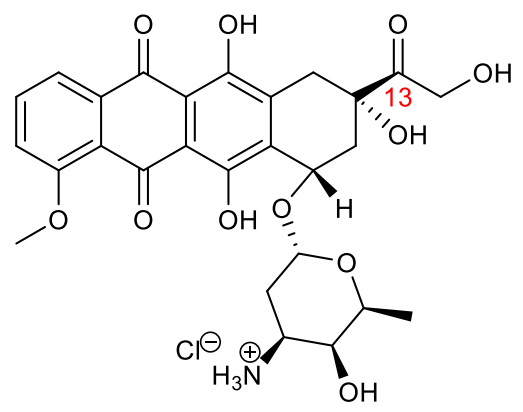

Dox

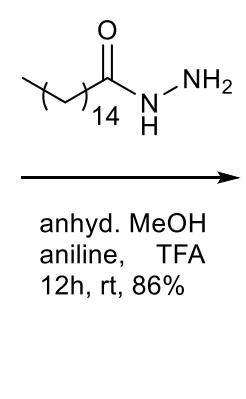

anhyd. $\mathrm{MeOH}$
aniline, TFA $12 \mathrm{~h}, \mathrm{rt}, 86 \%$

Scheme 4.2: Preparation of Doxorubicin hexadecanyl acyl hydrazone, Dox$\mathrm{AH}$

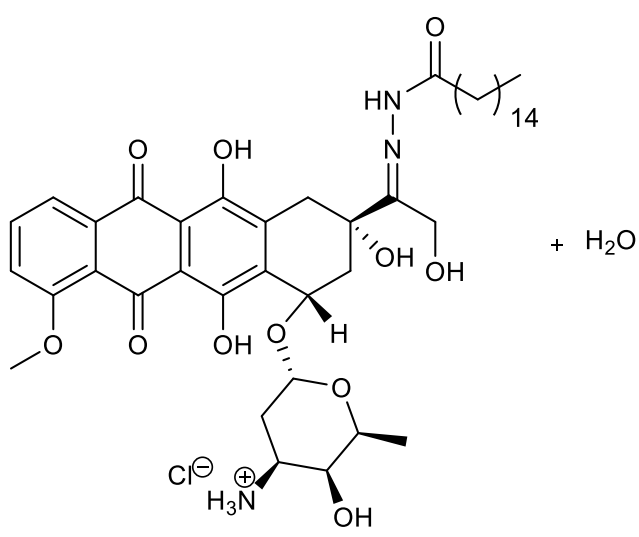

Dox-AH 


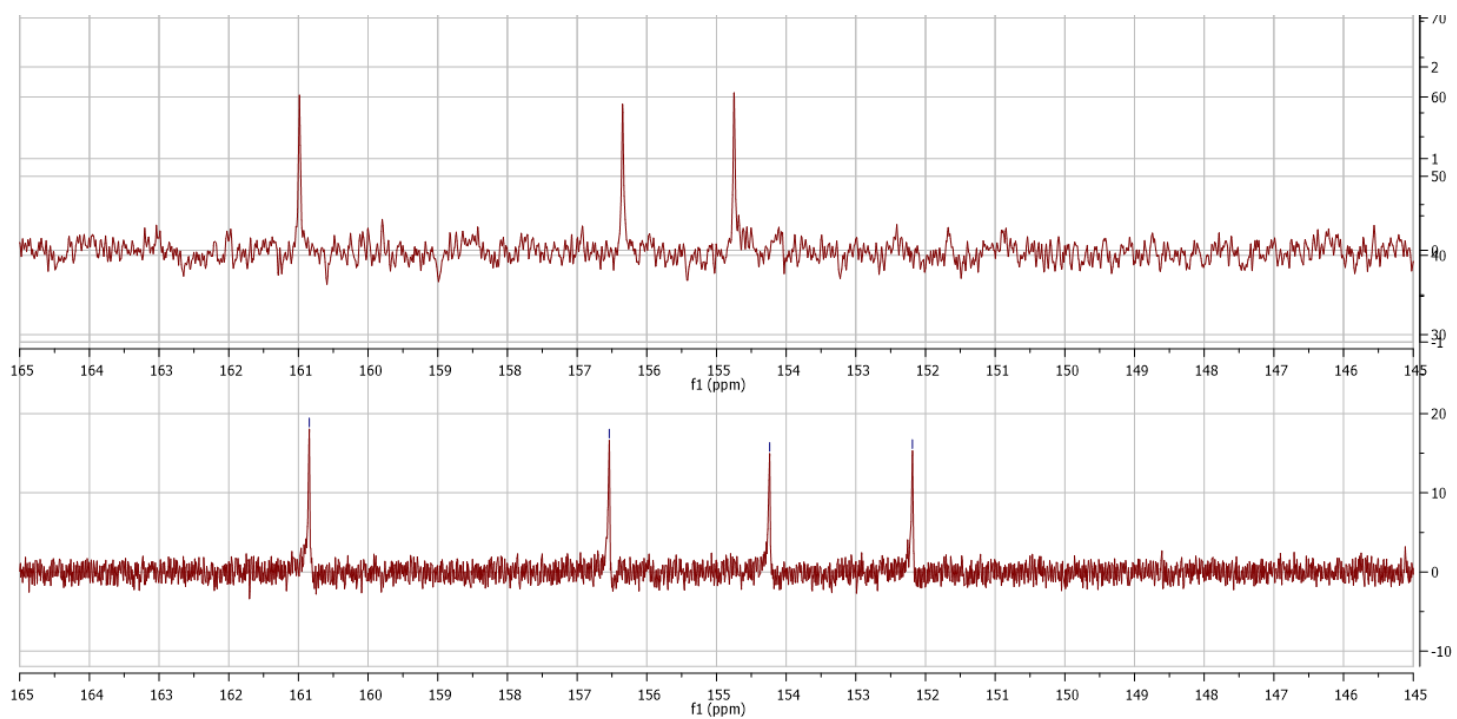

Figure 4.6. Stacked ${ }^{13} \mathrm{C}$ NMR of Dox (upper) and Dox-AH (lower), showing emergence of the $\mathrm{C}-13$ hydrazone chemical shift

\subsubsection{CML formation and stability}

To evaluate the formation and stability of CMLs, SNPs were stirred with cationic lipids 1, 2, or $\mathbf{3}$ in DMSO. Particle size was monitored over 24 hours mixing time by measuring dynamic light scattering (DLS). Lipids 2 and $\mathbf{3}$ with 14 and 18 carbon chains, respectively, did not form compact, stable CMLs (see appendix). However, lipid 1 with saturated C12 chains, formed small, stable CMLs averaging $67 \mathrm{~nm}$ in hydrodynamic diameter (Figure 4.7) and positive surface charge as indicated by zeta-potential of 31.72 +/- $2.40 \mathrm{mV}$. Consequently, lipid 1 was selected for use in subsequent studies. 


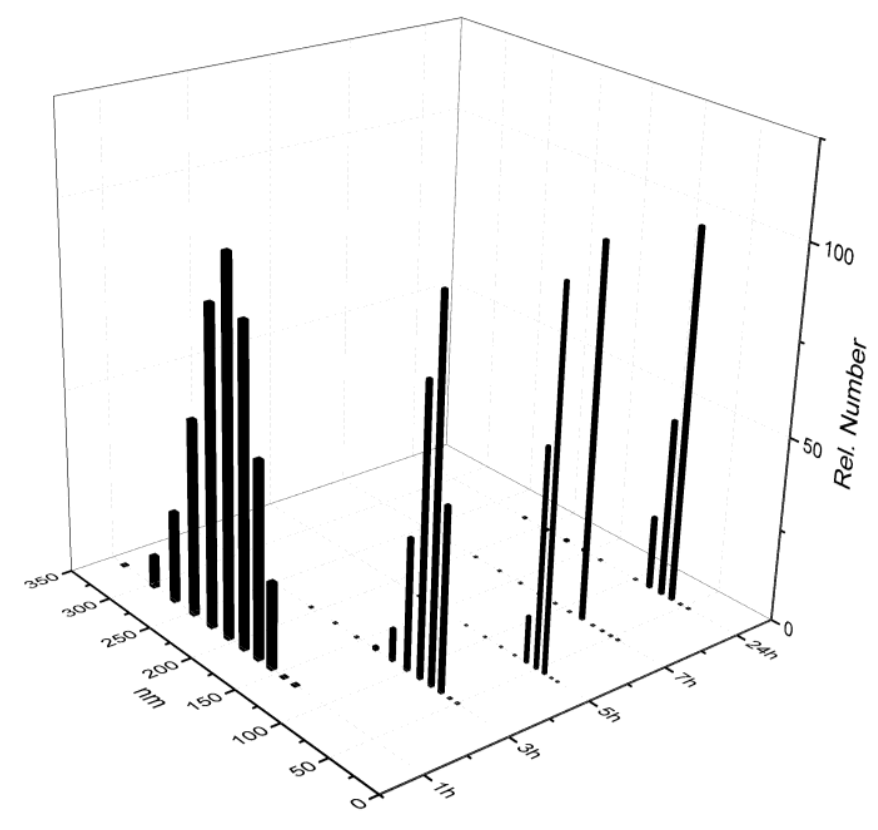

Figure 4.7. Dynamic light scattering measurements of CML diameter. The data represent direct measurements of a mixture of $13 \mu$ mols lipid 1 (Figure 4.2) with $1 \mathrm{mg}$ SNP in $2 \mathrm{~mL}$ DMSO taken over 24 hours mixing time.

\subsubsection{Titration of SNP with cationic lipid 1}

To determine the amount of lipid sufficient to coat SNPs with a bilayer, an aqueous solution of SNPs was titrated with increasing amounts of lipid 1 and the change in surface charge, as indicated by zeta-potential, was measured to determine a saturation point. A log-log plot of the data generated a sigmoidal curve with a plateau indicative of saturation of the particle (Figure 4.8). This zeta potential plateau represents approximately 28005700 lipid molecules per SNP (see appendix for calculation). In subsequent CML formulations, we aimed for a lipid amount just sufficient to reach the onset of the positive plateau of the charge titration curve to avoid using excess lipid. In the case of extruded MLs, a ratio of 4-13 mmol lipid/gm NPs is required to reach NP encapsulation efficiencies 
of $>70 \% .{ }^{157}$ By comparison, the charge titration curve for $\mathrm{CML}$ formation indicated that significantly less lipid is required, 0.2-0.5 mmol lipid 1/gm SNP.

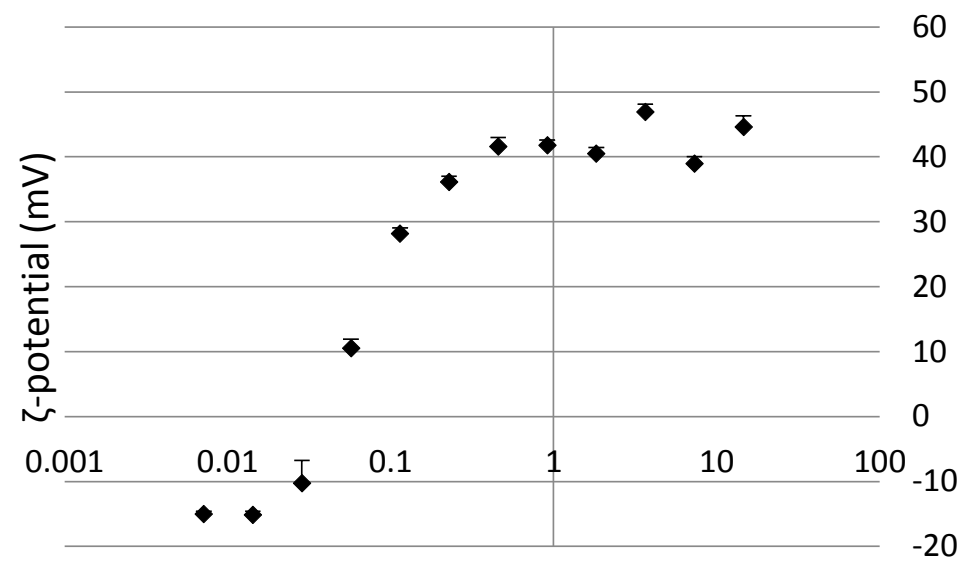

mmol lipid 1/g SNP

Figure 4.8. Zeta-potential of SNP titrated with lipid 1 in water.

\subsubsection{Estimation of lipid: Dox-AH ratio}

To determine the amount of Dox-AH to add for co-loading with lipid 1 onto SNPs, we examined the DLS of liposomal formulations of varying molar ratios of the two amphiphiles. We presumed that smaller liposomes would be indicative of tighter packing and greater stability. Liposomes were prepared using thin film hydration with sonication. ${ }^{158}$ DLS measurements were taken for each solution in water. Figure 4.9 shows the plot of the percent of Dox-AH added versus the diameter of the liposomes. From the data it was estimated that a Dox-AH percent less than 30 should be used for the sake of membrane stability. 


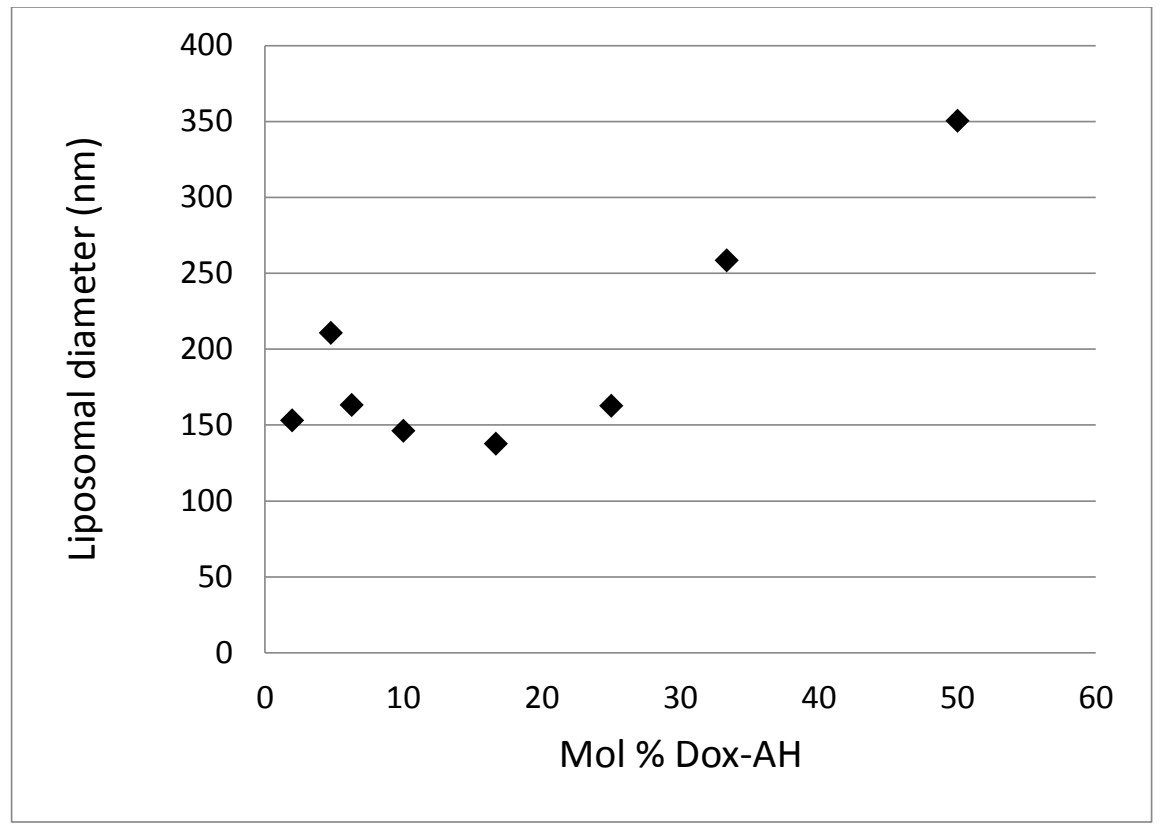

Figure 4.9. Diameter versus percent Dox-AH in a liposomal formulation with lipid 1.

\subsubsection{Cytotoxicity study}

Having established effective conditions for $\mathrm{CML}$ formation, we next examined incorporation of Dox-AH into CMLs to assess in vitro efficacy against MCF-7 breast cancer cells. Dr. Geoffrey Clark, a collaborator at the University of Louisville Medical School, kindly provided the use of his facilities, resources, and the assistance of his postdoctoral student, Dr. Katharine Hobbing, for this work.

Dox-AH-loaded CML were prepared by making a thin film of a 3:1 mixture of lipid 1 and Dox-AH. An aqueous dispersion of SNPs then was added to the lipid/Dox-AH film and the mixture was stirred for 12 hours to form loaded CMLs. Aliquots containing $50 \mu \mathrm{g}$ CMLs were diluted by $10 \%$ with $10 x$ PBS and the formulation was added directly to cells in growth medium (serum supplemented DMEM). Control conditions using separate constituents of the complete formulation were prepared similarly, including, as a positive 
control, aliquots of Dox and Dox-AH alone at 4 times the dose of the CML drug complexes. After $2 \mathrm{~h}$ incubation with treatments at $37^{\circ} \mathrm{C}$, the medium was removed and replaced with fresh medium. Cell viability (Figure 4.10) was assayed by trypan blue exclusion after 48 hours incubation. SNPs alone showed very low toxicity, as expected. Lipid 1 was somewhat cytotoxic but much more so when delivered in the form of CMLs, presumably a consequence of higher intracellular incorporation when associated with a cationic particle that is rapidly internalized. This carrier-mediated cytotoxicity, likely a result of cell membrane destabilization by intercalated cationic lipids, is undesirable, and future studies will examine how the effect can be mitigated. Nevertheless, the most potent formulations were the drug-loaded CMLs (both Dox and Dox-AH analogs), killing over $90 \%$ of the cells. It is noteworthy that the positive controls of Dox and Dox-AH alone displayed lower toxicity than the CML-drug complexes, despite being added at four times higher dose, again suggesting efficient $\mathrm{CML}$ internalization and thus intracellular accumulation of the cytotoxic cargo. To our surprise there was no significant difference between the cytotoxicity of Dox-AH CMLs and Dox CMLs. We had anticipated that the lipophilicity imparted by the added $\mathrm{C}_{16}$-acyl hydrazone would be necessary for CML-mediated conveyance of Dox into the cells. 


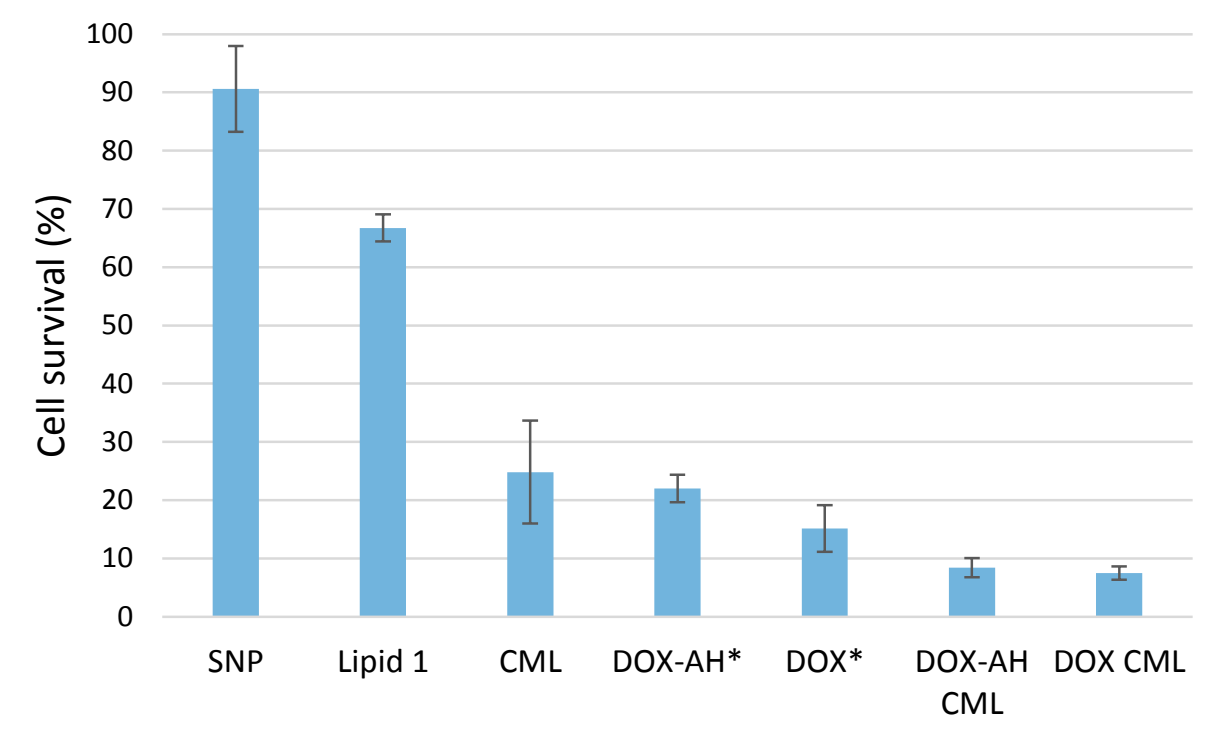

Figure 4.10. Cytotoxicity of treatment formulations in MCF-7 cells as indicated by trypan blue exclusion. Uncoated particles (SNP), Lipid 1 alone (Lipid 1), Lipid 1-coated SNP (CML), Dox-AH alone *administered at $4 \mathrm{X}$ higher drug dose than Dox-AH CML (Dox-AH), Dox alone *at 4X higher drug dose than Dox CML (Dox), Dox-AH-loaded CML (Dox-AH CML), and Doxloaded CML (Dox CML). Error bars represent standard error over biological triplicates.

To determine the degree to which the modification of Dox to acyl hydrazone Dox$\mathrm{AH}$ altered the drug-CML formulation as a whole, we turned to fluorescence imaging. Dox has a strong emission at $580 \mathrm{~nm}$, so fluorescence microscopy can be used to trace it within cells. Two hours after incubating MCF-7 cells, as above, with CML formulations containing either Dox-AH or Dox, fluorescence images were taken. The acquired images are paired with their bright-field counterparts in Figure 4.11. The more intense fluorescence was seen for the Dox-AH CML, so although the two drug-loaded CML function nearly identically with respect to toxicity, there is an apparent difference in the nature of their internalization. We hypothesize that Dox adsorbs to the SNP surface during CML formation via electrostatic interaction/hydrogen bonding from its ammonium sugar 
moiety and thus benefits from the uptake enhancement conferred by the $\mathrm{CML}$, but suffers from greater fluorescence quenching due to its tighter association with the SNP.
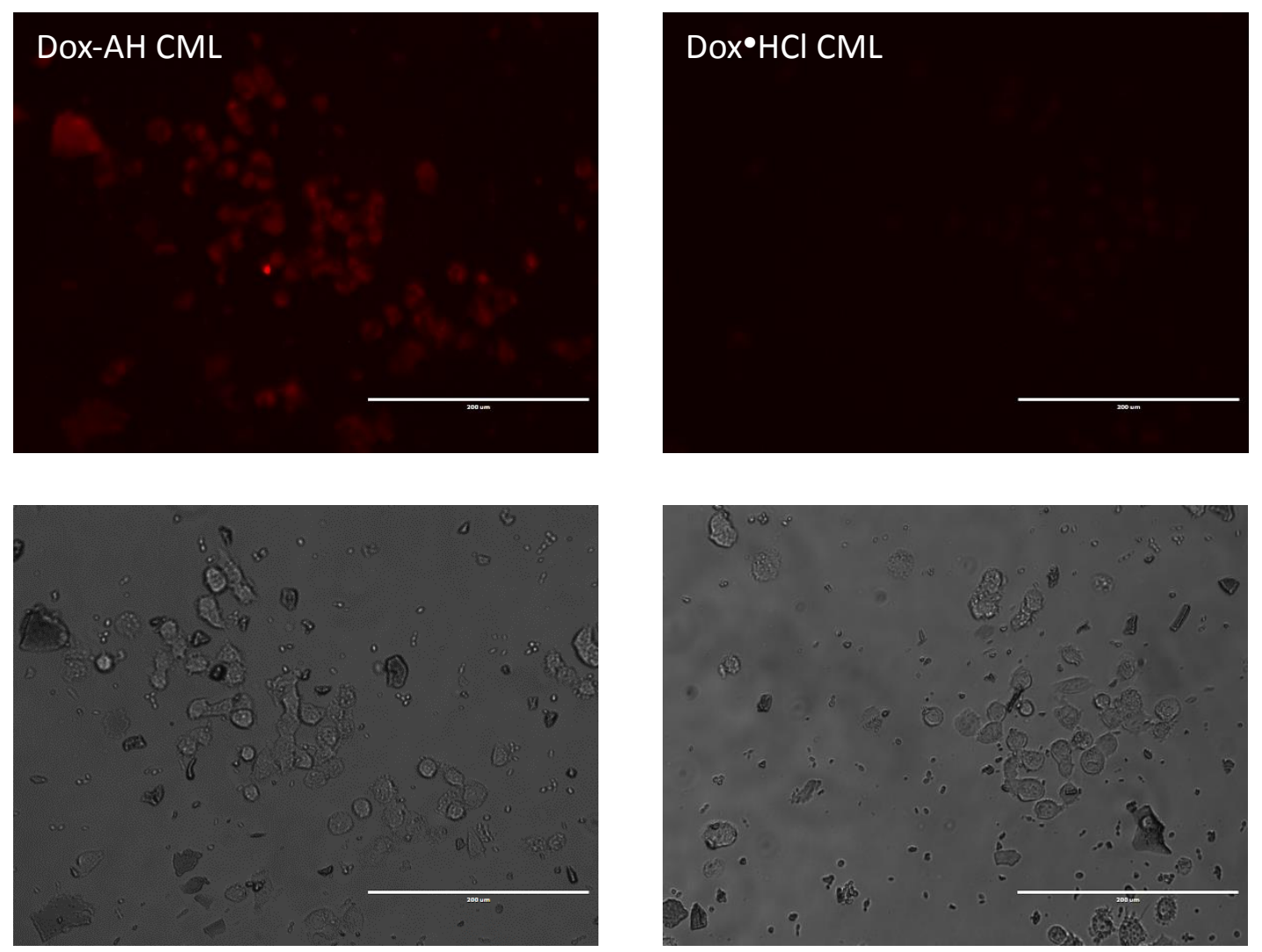

Figure 4.11. Stacked bright-field (lower) and fluorescence (upper) images of MCF-7 cells after two hours treatment with (left) Dox-AH-loaded CML, and (right) Dox-loaded CML. Scale: $200 \mu \mathrm{m}$.

Although unanticipated, we were gratified to find that CML formulations increased the efficacy of both hydrophilic and amphiphilic forms of our model drug. Still, for the purpose of Dox delivery by CML, an acyl hydrazone modification may yet be advantageous since Dox hydrazones have been shown to be more effective against multidrug resistant cell lines. 


\subsubsection{AMF and bilayer disruption}

A few investigators have examined the application of AMF to lipid-coated NPs for achieving substrate release. Hsu and coworkers, for example, prepared "solid lipid" nanostructures with embedded NP. They were constructed with a lipid core of triglyceride and an encapsulating polymeric surfactant. Embedded within the hydrophobic interior were NPs and a model drug. Application of AMF at $25 \mathrm{kHz}$ for 20 minutes resulted in release of $35 \%$ of the embedded drug. ${ }^{93}$ Similarly, Bringas et al. ${ }^{92}$ reported AMF-mediated release of a dye from a lipid-coated SNP system. In this work, NPs were capped with mesoporous silica into which the dye methylene blue was incorporated. The mesoporous silica was then coated with a lipid bilayer consisting of 1,2-dioleoyl-sn-glycero-3-phosphocholine (DOPC). They observed full release of the dye after AMF exposure at $50 \mathrm{~Hz}$ for 350 minutes. These reports encouraged us to think that AMF application might be used to induce Dox release in our CML system. However several toxicity trials failed to show a difference in cell survival for treatment conditions that incorporated AMF exposure. The studies mentioned previously differ from our system in that the AMF frequency used was lower than our own, which is fixed by the size of the coil on our generator. Additionally, both involve structures that contain multiple NPs. We theorize that these factors may have lessened the impact of AMF exposure on substrate release in our system. 


\subsection{Gene transfection studies}

As noted in the opening of this chapter, cationic lipids are commonly utilized as synthetic gene vectors owing to their ability to condense nucleic acids and form cationic lipoplexes that are rapidly taken up by cells through endocytosis. Given the similar but distinct structures of the oxime ether lipids prepared for the CML study as compared to those that had previously proven ${ }^{141}$ to be efficient transfection agents (Figure 4.12), we chose to examine these novel compounds for their potential for nucleic acid delivery. We were especially interested to see the effect that the 1,2 diol component of lipids $\mathbf{2}$ and $\mathbf{3}$ might have on transfection efficiency, as recent reviews of structure-activity relationships in cationic lipids had cited enhanced transfection for lipids with hydroxylated headgroups. ${ }^{146,159}$

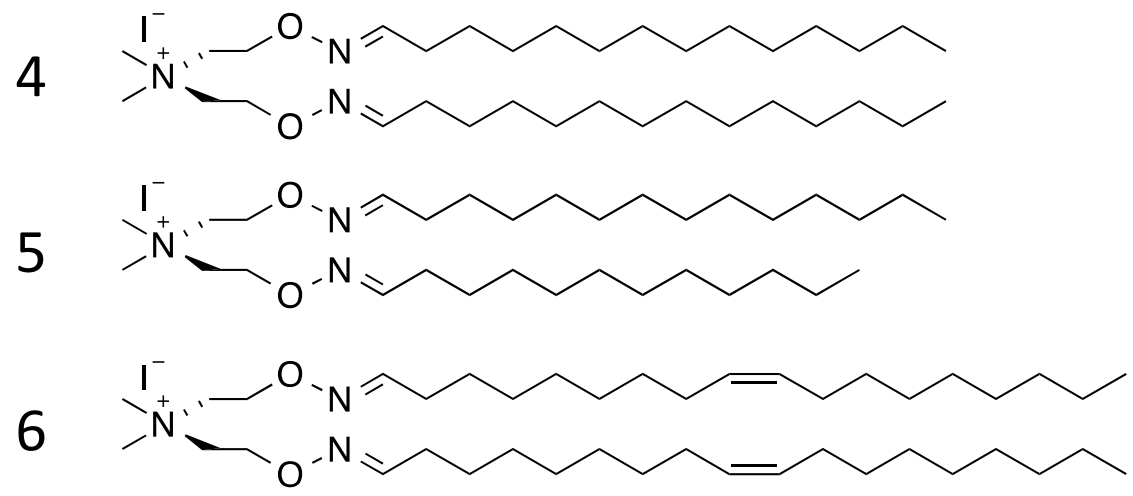

Figure 4.12. First generation oxime ether lipids for gene transfection

In work done by collaborators at the National Cancer Institute, lipids 2 and 3 were evaluated and compared to the previous set of transfection lipids (4, 5, and 6) for 
DNA/RNA binding efficiency, transfection efficiency, cell viability, and RNA release. In this section, the results related to lipids $\mathbf{2}$ and $\mathbf{3}$ are featured.

\subsubsection{Binding affinity}

Liposomes were formed from each lipid in combination with a zwitterionic co-lipid (DOPE) and the binding affinity of the liposomes for a fluorescently tagged hybrid DNA/RNA was studied by fluorescence anisotropy, a technique that measures changes in polarization/tumbling time. In this assay, lipids $\mathbf{2}$ and $\mathbf{3}$ were shown to bind nucleic acids as efficiently as or more so than the first generation lipids (Figure 4.13).

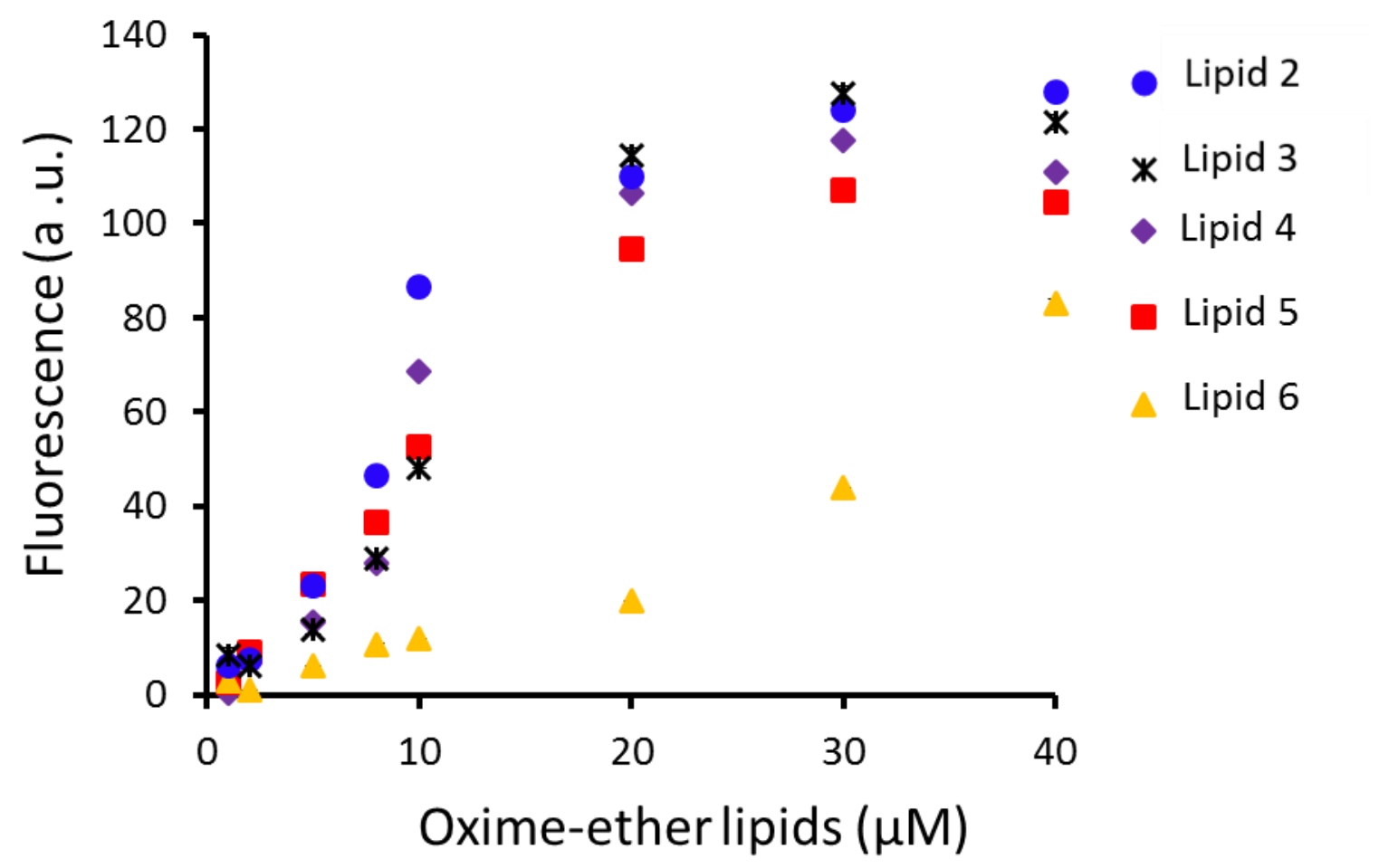

Figure 4.13. Binding affinity of liposomes at different concentrations (1-40 $\mu \mathrm{M})$ with $100 \mathrm{nM}$ of Alexa $488 \mathrm{RNA} / \mathrm{DNA}$ hybrid as measured by fluorescence anisotropy 


\subsubsection{Transfection efficiency}

The transfection efficiency of the lipids was then assessed using the fluorescently labeled hybrid RNA/DNA in MDA-MB-231 breast cancer cells. The results, depicted in Figure 4.14, show a concentration dependence, where lipid 2 showed the highest transfection efficiency at lower doses and lipid $\mathbf{3}$ showed the highest efficiency at higher doses. In either case, the hydroxylated lipids performed well in comparison to their first generation analogs.

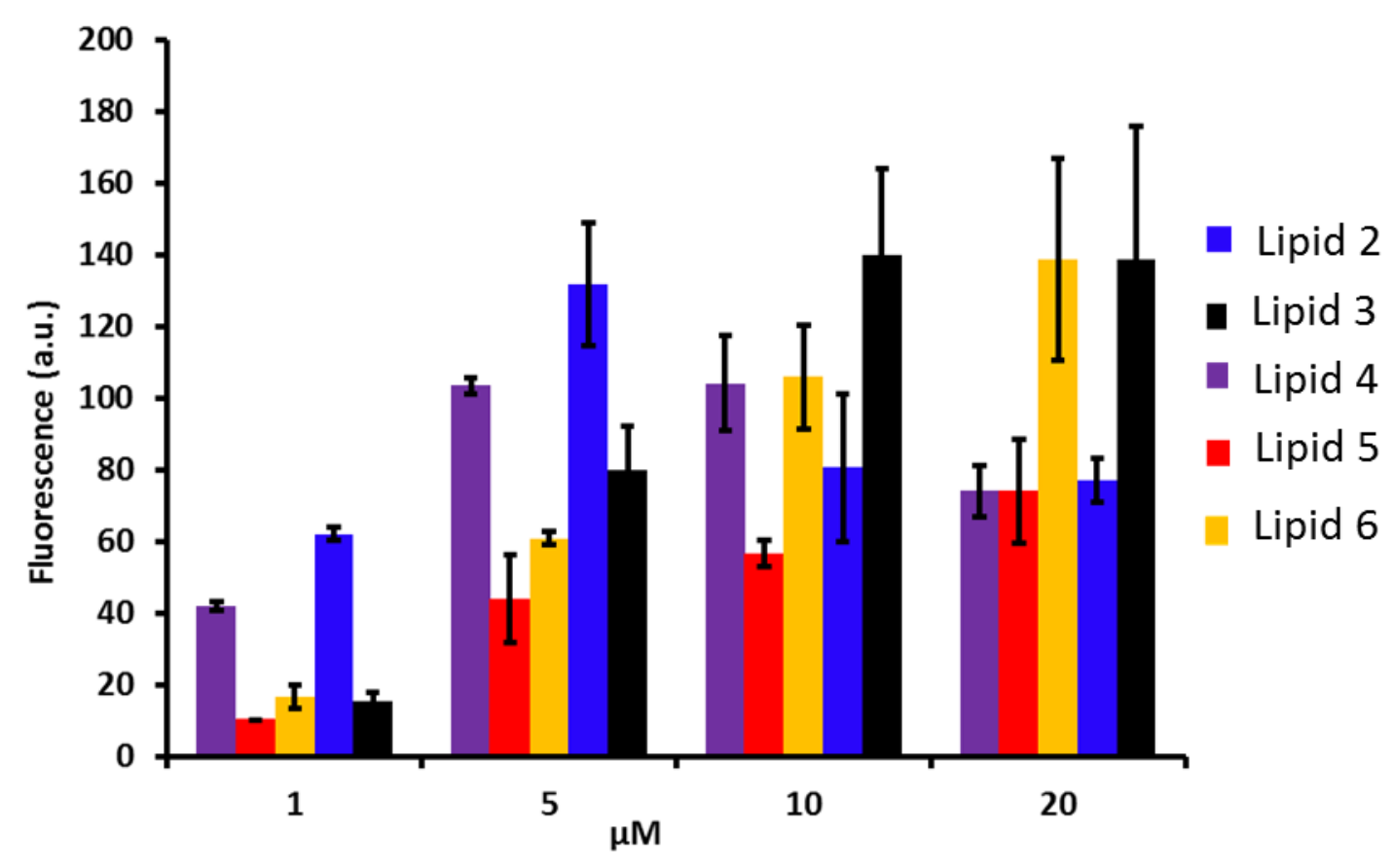

Figure 4.14. Transfection efficiency of oxime ether lipids with Alexa 488tagged hybrid DNA/RNA 


\subsubsection{Cell viability}

Cell viability was assessed 24 hours after treatment of MDA-MB-231 cells with liposomes of each lipid by fluorescence of CellTitre blue reagent, a dye (resazurin) which becomes fluorescent by enzymatic conversion to resorufin in live cells. None of the lipids elicited significant reduction in viability as shown in Figure 4.15.

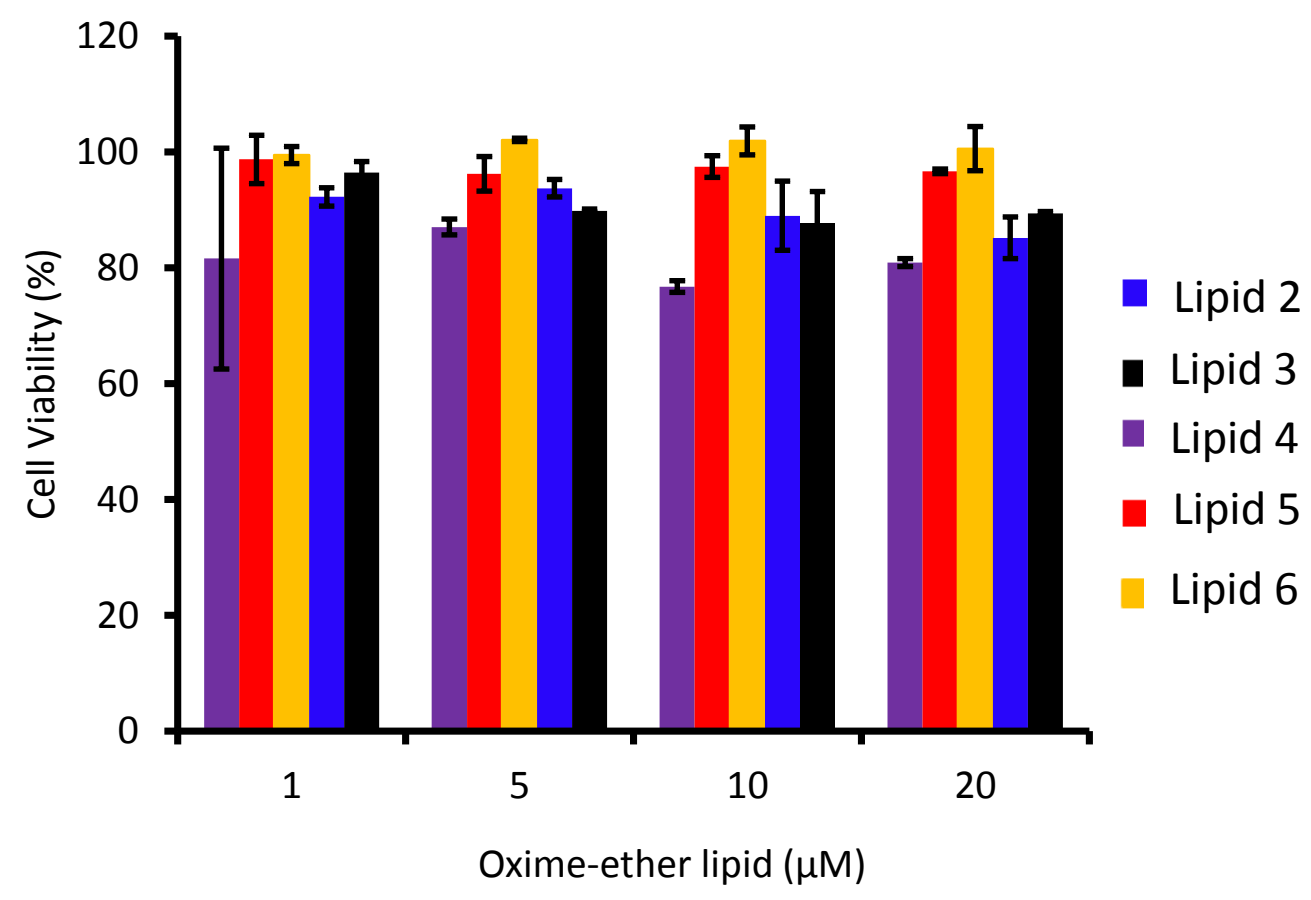

Figure 4.15. Viablilty of MDA-MB-231 cells with liposome/RNA complexes by CellTitre blue assay

\subsubsection{Gene expression}

Gene silencing was used to assess nucleic acid release and gene expression for cells transfected with lipoplexes of each lipid. In this examination, MDA-MB-231 cells expressing green fluorescent protein (GFP) were prepared, along with lipoplexes of lipids paired with anti-GFP dicer substrate (DS) RNA. Gene expression was then evaluated 
based upon the silencing of the fluorescent protein (Figure 4.16A). Lipids 2 and 3 demonstrated silencing of the GFP gene indicating release of dicer RNA. In this regard, lipid 3 bested the other analogs. Figure 4.16B shows the reduction in green fluorescence for the lipoplex of lipid 3.

A

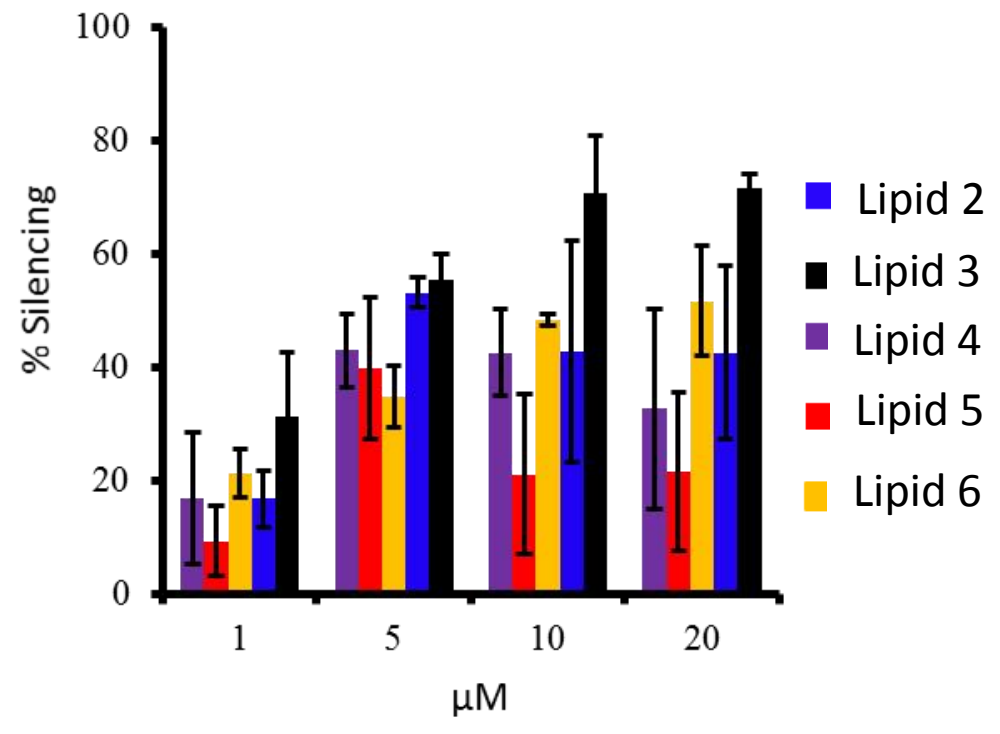

B

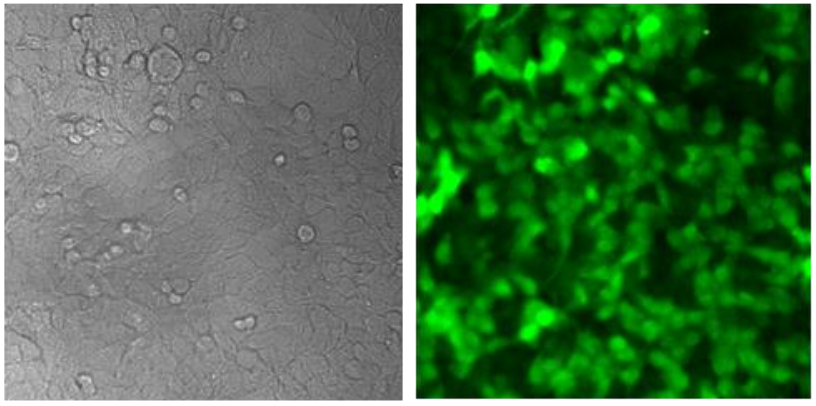

Lipid 3/DS RNA

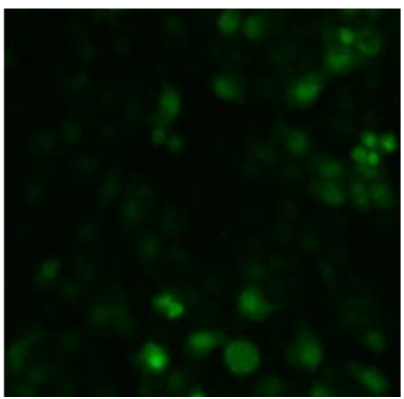

Figure 4.16. (a) Silencing of GFP by lipoplexes of lipids with DS RNA (b) Fluorescent microscope images of MDA-MB-231/GFP cells showing silencing of GFP by DS RNA lipoplex with lipid 3 
The results of the transfection studies indicated that the hydroxylated cationic oxime ether lipids are promising candidates for non-viral nucleic acid delivery. In our examination of the relationship between lipid structure and siRNA transfer efficacy, some trends emerged. Polar domain hydroxylation had the greatest influence on binding and release properties, where hydroxylated lipids $\mathbf{2}$ and $\mathbf{3}$ outperformed their nonhydroxylated counterparts both in regard to tighter binding, based on fluorescence anisotropy measurements, as well as in nucleic acid release on cellular internalization, as indicated by GFP silencing. Whereas tight lipid complexation with nucleic acids is desirable for protection against enzymatic degradation, the nucleic acid phosphate-lipid ammonium interactions ${ }^{160}$ in concert with hydrophobic domain packing and any polar domain hydrogen bonding interactions must be readily overcome for later stage lipoplex disassembly to occur. In this regard, the hydroxylated lipids appear well-structured for effective siRNA protection and release. Hydrophobic domain dissymmetry has been suggested as a structural factor that is beneficial for nucleic acid release, thus ultimately enhancing transfection activity. ${ }^{161-162}$ All of the lipids in these studies were used as a mixture of $(E, E)$ - and $(E, Z)$-oxime ether isomers, meaning a nontrivial amount of nonsymmetric $(E, Z)$-lipid was present. Non-symmetric hydrophobic domains likely benefit transfection due to better DNA release through looser lipid packing in the lipoplex formulation. ${ }^{163-164}$

Lipoplex uptake and activity also was facilitated by lipid hydroxylation, and this observation agrees well with the assessment of a recent review ${ }^{146}$ citing greater 
transfection efficiencies for lipids possessing hydroxylated headgroups. This effect is thought to be mediated by the decrease in headgroup hydration that results from the incorporation of functionality capable of hydrogen bonding. Neighboring lipid-lipid interactions in the lipoplex bilayers lead to exclusion of interstitial water, and the resulting high positive charge/low hydration motif promotes electrostatic interactions not only with the polynucleotide but also with cell membranes. ${ }^{165}$

Lipid 3 was arguably the frontrunner in these studies where the unsaturation in the hydrophobic domain appeared to enhance gene expression. Recent findings may explain this behavior; unsaturated lipids are thought to fluidize endosomal bilayers, facilitating lipoplex escape and have also been correlated with better nuclear localization. ${ }^{159}$

\subsection{Conclusions}

Novel oxime either lipids were synthesized with the aim of optimizing their ability to self-assemble into bilayers surrounding silica-coated magnetic NPs. The resultant cationic magnetoliposomes were assessed for their ability to function in vitro as drug carriers in a preliminary study with Dox and a modified amphiphilic analog. Association of the drugs with the CML increased the potency of the nanoassembly over that of the free drug. The preparation of the CML-drug complex was accomplished by simple mixing of SNPs (room temp., $\mathrm{H}_{2} \mathrm{O}$, neutral $\mathrm{pH}$ ) with small quantities of lipid and drug: 0.3-0.4 mg lipid + .085-0.12 mg anthracycline per mg SNP. The CML-drug formulations showed high toxicity to MCF-7 cancer cells after 2 hours exposure to doses of $50 \mu \mathrm{g}$ SNP/>50,000 cells 
(3.6 $\mu \mathrm{M}$ with respect to the anthracycline). The CML-drug formulations functioned effectively in the presence of serum-supplemented cell growth medium. These results point to the potential for cationic magnetoliposomes built on $\mathrm{Fe}_{3} \mathrm{O}_{4}-\mathrm{SiO}_{2}$ nanoparticles as magnetic drug delivery vehicles, thus providing opportunity for use of the tandem techniques of magnetic targeting, imaging, and inductive heating. Further, in vitro assessment of the transfection activity of hydroxylated oxime ether lipids and a comparison with similar lipids of that class highlighted their distinctive efficacy as gene delivery agents. 


\section{CHAPTER 5 EXPERIMENTAL PROCEDURES}

5.1. General Procedures

5.2. Experimental Procedures for Chapter 2

5.3. Experimental Procedures for Chapter 3

5.4. Experimental Procedures for Chapter 4 


\subsection{General Procedures}

Reagents were typically purchased from Aldrich Chemical Company (Milwaukee, WI). Reagent grade ACS dichloromethane, tetrahydrofuran (THF), acetone, hexanes, ethyl acetate (EtOAc), and diethyl ether were purchased from Pharmco-AAPER (Shelbyville, Ky). THF and $\mathrm{CH}_{2} \mathrm{Cl}_{2}$ were purified, when indicated, using a SPBT-101 Bench Top Solvent Purification System (LC Technology Solutions, Inc., Salisbury, MA). $\mathrm{CDCl}_{3}$ (99.8\%) was purchased from Cambridge Isotope Laboratories (Andover, MA). NMR spectra were acquired on a Varian Inova $500 \mathrm{MHz}$ or Varian $400 \mathrm{MHz}$ spectrometer, as indicated. Spectra were referenced to the residual solvent peaks according to Gottlieb et al. ${ }^{166}$ FTIR spectra were acquired on a Perkin Elmer Spectrum 100 FT-IR Spectrometer (diamond crystal) with range: $4000-650 \mathrm{~cm}^{-1}$, resolution: $4 \mathrm{~cm}^{-1}$, interval: $1 \mathrm{~cm}^{-1}$, and scans: 4. Solid samples were scanned using an ATR attachment, liquid samples were scanned neat or after being dissolved in $\mathrm{CH}_{2} \mathrm{Cl}_{2}$, placing a droplet onto the crystal, and allowing the solvent to evaporate. Alternatively, DRIFT IR spectra were acquired on a Mattson galaxy series 5000 DRIFT spectrometer using 64 scans, at a scan speed of $2.1 \mathrm{kHz}$ and a resolution of $4 \mathrm{v}$. DRIFT samples were prepared as a loose powder in $\mathrm{KBr}$. High resolution mass spectra were acquired on a Finnigan LTQ- FT spectrometer (Thermo Electron Corp). MALDI-TOF-MS detection was done on a Voyager DE-Pro MALDI-TOF instrument (PE Biosystems) using 2,5-dihydroxybenzoic acid (2,5-DHB) matrix. Melting points were determined using a Fisher-Johns melting point apparatus or an SRS Digimelt. Heating of cell extract samples was done on a Barnstead/Thermolyne Type 17600 Dribath. Centrifugation was performed on an Eppendorf Centrifuge type 5415R; vacuum 
centrifugation was performed on an Eppendorf Vacufuge. Reaction progress was monitored by thin layer chromatography (silica gel $60 \AA$ F-254 plates) using UV visualization and staining with ninhydrin, phosphomolybdic acid, or $p$-anisaldehyde solutions. Column chromatography was performed using silica gel (230-400 mesh) or by using a Teledyne Isco combiflash system. Cell microscopy was done on an EVOS FL Cell Imaging System (Advanced Microscopy Group, WA). Dynamic light scattering and Zpotential measurements were acquired on a Brookhaven ZetaPALS Zeta Potential analyzer. 


\subsection{Experimental Procedures for Chapter 2}

5.2.1 Syntheses of reagents and intermediates

5.2.2 Mass spectrometry methods

5.2.3 Extraction solvent determination, counterion comparison, and partition efficiency

5.2.4 Verification of linear instrument response

5.2.5 Cell culture and extract preparation

5.2.6 Cell extract derivatization

5.2.7 Data analysis and statistics

5.2.8 Estimation of cellular suppression factor and quantification of pyruvate 


\subsection{Experimental Procedures for Chapter 2}

\subsubsection{Syntheses of reagents and intermediates}<smiles>CN(C(I)CO)C(C)(C)C(C)(C)C</smiles>

$\mathrm{N}$-Hydroxyethyl- $\mathrm{N}, \mathrm{N}$-dimethyl-octylammonium iodide (2.1)

Following a previously reported protocol, ${ }^{167}$ a solution of $N, \quad N-$ dimethylethanolamine $(0.50 \mathrm{~mL}, 4.98 \mathrm{mmol})$ and iodooctane $(0.099 \mathrm{~mL}, 5.47 \mathrm{mmol})$ in acetone $(15 \mathrm{~mL})$ were heated to reflux. After $30 \mathrm{~h}$, the reaction was cooled to room temperature and dried by rotary evaporation. To the resultant yellow solid, hexanes were added $(6 \mathrm{~mL})$ and the solution was triturated with sonication until the solid dispersed. Buchner filtration of the suspension followed by vacuum drying of the collected solids yielded 2.1 (1.77 g, 92\%) as white crystals; mp 76-78 ${ }^{\circ} \mathrm{C} ;{ }^{1} \mathrm{H}-\mathrm{NMR}\left(500 \mathrm{MHz}, \mathrm{CDCl}_{3}\right) \delta 0.82$ $(\mathrm{t}, J=6.7 \mathrm{H}, 3 \mathrm{H}) ; 1.24(\mathrm{~m}, 10 \mathrm{H}) ; 1.73(\mathrm{~m}, 2 \mathrm{H}) ; 3.32(\mathrm{~s}, 6 \mathrm{H}) ; 3.52(\mathrm{~m}, 2 \mathrm{H}) ; 3.71(\mathrm{~m}, 2 \mathrm{H}) ; 4.08$ (m, 3H); ${ }^{13} \mathrm{C}$ NMR (125 MHz, $\left.\mathrm{CDCl}_{3}\right)$ 11C $\delta 14.1,22.5,22.9,26.2,29.0,29.1,31.6,52.4$; $55.5,65.5,66.2$. IR 3277, 2952, 2920, 2853, 1483, 1464, 1410, 1104, 1073, 1006, 968, 938, 843, 728, $594 \mathrm{~cm}^{-1}$; HRMS m/z calcd $\left[\mathrm{C}_{12} \mathrm{H}_{28} \mathrm{NO}\right]^{+}$202.2165, observed 202.2166. 


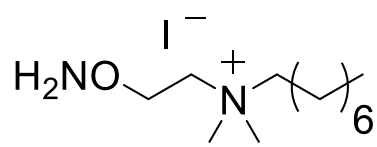

$N$-Aminooxyethyl- $N, N$-dimethyl-octylammonium iodide (QOA)

To a solution of alcohol 2.1 (1.50 g, $4.55 \mathrm{mmol}), N$-hydroxyphthalimide ( $0.817 \mathrm{~g}$, $5.01 \mathrm{mmol})$ triphenylphosphine $(1.31 \mathrm{~g}, 5.00 \mathrm{mmol})$ in THF $(15 \mathrm{~mL})$ at $\mathrm{rt}$ was added diisopropyl azodicarboxylate (DIAD) $(0.993 \mathrm{~mL}, 5.01 \mathrm{mmol})$. After stirring $12 \mathrm{~h}$ at room temperature, the solvents were removed by rotary evaporation. To the crude yellow residue was added ethyl acetate $(7.0 \mathrm{~mL})$. The resultant suspension of white solids was filtered, and the retentate (phthalimide intermediate) was used directly in the next step. ${ }^{1} \mathrm{H}$ NMR $\left(500 \mathrm{MHz}, \mathrm{CDCl}_{3}\right) \delta 0.85(\mathrm{t}, J=6.5 \mathrm{~Hz}, 3 \mathrm{H}) ; 1.32(\mathrm{~m}, 10 \mathrm{H}) ; 1.85(\mathrm{~m}, 2 \mathrm{H}) ; 3.57(\mathrm{~s}$, $6 \mathrm{H}) ; 3.78(\mathrm{~m}, 2 \mathrm{H}) ; 4.37(\mathrm{~m}, 2 \mathrm{H}) ; 4.74(\mathrm{~m}, 2 \mathrm{H}) ; 7.80(\mathrm{~m}, 2 \mathrm{H}) ; 7.85(\mathrm{~m}, 2 \mathrm{H})$.

To the phthalimide protected salt $(1.88 \mathrm{~g}, 3.96 \mathrm{mmol})$ in $\mathrm{CH}_{2} \mathrm{Cl}_{2}$ was added hydrazine monohydrate $(0.59 \mathrm{~g}, 11.9 \mathrm{mmol})$ and the mixture was stirred at $\mathrm{rt}$ for $2 \mathrm{~h}$. Collection of the $\mathrm{CH}_{2} \mathrm{Cl}_{2}$ filtrate followed by drying yielded QOA.I (1.26 gm, 93\%) as a white powder; mp 55.5-58.6 ${ }^{\circ} \mathrm{C} .{ }^{1} \mathrm{H}$ NMR $\left(500 \mathrm{MHz}, \mathrm{CDCl}_{3}\right) \delta 0.80(\mathrm{t}, J=6.5 \mathrm{~Hz}, 3 \mathrm{H}) ; 1.24(\mathrm{~m}$, $10 \mathrm{H}) ; 1.69(\mathrm{~m}, 2 \mathrm{H}) ; 3.33(\mathrm{~s}, 6 \mathrm{H}) ; 3.51(\mathrm{~m}, 2 \mathrm{H}) ; 3.84(\mathrm{~m}, 2 \mathrm{H}) ; 4.12(\mathrm{~m}, 2 \mathrm{H}) ; 4.95\left(\mathrm{bs}, 2 \mathrm{H}-\mathrm{NH}_{2-}\right.$ ). Key ${ }^{1} \mathrm{H}^{1}{ }^{1} \mathrm{H}$ COSI correlations: $\delta$ 4.12-3.84 $\left(\mathrm{C}\left(1^{\prime}\right) \mathrm{H}_{2}-\mathrm{C}\left(2^{\prime}\right) \mathrm{H}_{2}\right) ; 3.51-1.69\left(\mathrm{C}(1) \mathrm{H}_{2}-\mathrm{C}(2) \mathrm{H}_{2}\right)$. ${ }^{13} \mathrm{C}$ NMR $\left(125 \mathrm{MHz}, \mathrm{CDCl}_{3}\right) 11 \mathrm{C} \delta 14.0,52.2 ; 22.5,22.9,26.2,28.9,29.1,31.6,62.5,65.8$, 69.0. IR: $3273,3029,2927,2855,1472,1172,954$ v(O-N), 858, $495 \mathrm{~cm}^{-1}$. HRMS m/z calcd $\left[\mathrm{C}_{12} \mathrm{H}_{29} \mathrm{~N}_{2} \mathrm{O}\right]^{+}$217.227439, observed 217.22779. 
<smiles></smiles>

Dodecyl(2-hydroxyethyl)dimethylazanium bromide (2.3)

A solution of $\mathrm{N}, \mathrm{N}$-dimethylethanolamine $(0.5 \mathrm{~mL}, 4.97 \mathrm{mmol})$ and 1 bromododecane $(1.31 \mathrm{~mL}, 5.47 \mathrm{mmol})$ in acetone $(25 \mathrm{~mL})$ were heated to reflux, After $24 \mathrm{~h}$, the reaction was cooled to $\mathrm{rt}$ and the precipitated solids were collected by filtration, washed with chilled acetone and dried under vacuum. The reaction filtrate was dried under rotary evaporation and to the resulting yellow residue hexane was added. Trituration with sonication of the hexane produced a white precipitate which was collected by filtration and combined with the precipitate from acetone to afford 2.3 (0.977 gm, $58 \%$ ) as white crystals; $\mathrm{mp}$ (decomp) $190{ }^{\circ} \mathrm{C} .{ }^{1} \mathrm{H} \mathrm{NMR}\left(500 \mathrm{MHz}, \mathrm{CDCl}_{3}\right), \delta 0.87$ $(\mathrm{t}, J=6.75 \mathrm{~Hz}, 3 \mathrm{H}) ; 1.25(\mathrm{~m}, 18 \mathrm{H}) ; 1.75(\mathrm{~m}, 2 \mathrm{H}) ; 3.37(\mathrm{~s}, 6 \mathrm{H}) ; 3.54(\mathrm{~m}, 2 \mathrm{H}) ; 3.75(\mathrm{~m}, 2 \mathrm{H})$; $4.15(\mathrm{~m}, 2 \mathrm{H}) ; 4.99(\mathrm{t}, J=5.5 \mathrm{~Hz}, 1 \mathrm{H}) .{ }^{13} \mathrm{C}-\mathrm{NMR}\left(125 \mathrm{MHz}, \mathrm{CDCl}_{3}\right) \delta 15 \mathrm{C} \delta$ 14.1; 22.6; 22.9; 26.2; 29.2; 29.3; 29.4; 29.5; 29.6 (2 signals); 31.8; 52.0, 55.9, 65.4, 66.1. IR: 3248, 3006, $2952,2917,2850,1475,1421,1375,1078,1073,1055,1016,965,918,900,730,718$ $601,571,432 \mathrm{~cm}^{-1}$. 
<smiles></smiles>

$\mathrm{N}$-Aminooxyethyl- $\mathrm{N}, \mathrm{N}$-dimethyl-dodecylammonium bromide (QDA·Br)

To a solution of alcohol 2.3 (0.699 g, $2.06 \mathrm{mmol})$, N-hydroxyphthalimide $(0.371 \mathrm{~g}$ $2.27 \mathrm{mmol})$, triphenylphosphine $(0.596 \mathrm{~g}, 2.27 \mathrm{mmol})$ in $\mathrm{CH}_{2} \mathrm{Cl}_{2}(10 \mathrm{~mL})$ at $\mathrm{rt}$ was added DIAD (0.45 mL, $2.27 \mathrm{mmol})$. After stirring for $15 \mathrm{~h}$ at $\mathrm{rt}$, the solvents were removed by rotary evaporation. To the crude yellow residue was added ethyl acetate $(20 \mathrm{~mL})$. The resultant suspension of white solids was filtered, and the retentate (phthalimide intermediate) was used directly in the next step. ${ }^{1} \mathrm{H}-\mathrm{NMR}\left(500 \mathrm{MHz}, \mathrm{CDCl}_{3}\right) \delta 0.86(\mathrm{t}, \mathrm{J}=$ $6.7 \mathrm{~Hz}, 3 \mathrm{H}) ; 1.30(\mathrm{~m}, 18 \mathrm{H}) ; 1.83(\mathrm{~m}, 2 \mathrm{H}) ; 3.56\left(\mathrm{~s}, 6 \mathrm{H}-\mathrm{N}(\mathrm{Me}) 2^{-}\right) ; 3.76(\mathrm{~m}, 2 \mathrm{H}) ; 4.41(\mathrm{~m}, 2 \mathrm{H})$; $4.74(\mathrm{~m}, 2 \mathrm{H}) ; 7.82(\mathrm{~m}, 2 \mathrm{H}) ; 7.84(\mathrm{~m}, 2 \mathrm{H})$.

To the phthalimide protected salt $(1.93 \mathrm{~g}, 3.98 \mathrm{mmol})$ in anhydrous ethanol was added hydrazine monohydrate $(1.24 \mathrm{~g}, 24.7 \mathrm{mmol})$. The reaction was stirred for $6 \mathrm{hr}$ at $50^{\circ} \mathrm{C}$. The ethanol was then removed by rotary evaporation and $\mathrm{CH}_{2} \mathrm{Cl}_{2}$ was added (17 $\mathrm{mL}$ ). The resulting white precipitate was filtered over a Buchner funnel and washed with $\mathrm{CH}_{2} \mathrm{Cl}_{2}$. The $\mathrm{CH}_{2} \mathrm{Cl}_{2}$ filtrate was dried by rotary evaporation. To the resultant off-white residue toluene was added $(30 \mathrm{~mL})$, heating and sonication yielded a suspension of fine white particles which were collected over a glass Buchner funnel. This afforded QDA·Br as a white powder; $\mathrm{mp}$ (decomp) $187{ }^{\circ} \mathrm{C} .{ }^{1} \mathrm{H}-\mathrm{NMR}\left(500 \mathrm{MHz}, \mathrm{CDCl}_{3}\right) \delta 0.84(\mathrm{t}(\mathrm{J}=6.7 \mathrm{~Hz})$, 3H); $1.27(\mathrm{~m}, 18 \mathrm{H}) ; 1.70(\mathrm{~m} \mathrm{2H}) ; 3.36(\mathrm{~s}, 6 \mathrm{H}) ; 3.54(\mathrm{~m}, 2 \mathrm{H}) ; 3.94(\mathrm{~m}, 2 \mathrm{H}) ; 4.17(\mathrm{~m}, 2 \mathrm{H}) ; 5.30$ (bs, $2 \mathrm{H}) .{ }^{13} \mathrm{C}-\mathrm{NMR}\left(125 \mathrm{MHz}, \mathrm{CDCl}_{3}\right) 15 \mathrm{C} \delta$ 14.1; 22.1 (2 signals); 22.7; 22.9; 26.3; 29.2; 29.3; 29.5; 29.6; 31.9; 51.8, 62.2, 65.6. IR: 3280, 2921, 2848, 1653, 1576, 1472, 1173, 
1073, $954 \mathrm{v}(\mathrm{O}-\mathrm{N}), 719,494 \mathrm{~cm}^{-1}$. HRMS $\mathrm{m} / z$ calcd $\left[\mathrm{C}_{16} \mathrm{H}_{37} \mathrm{~N}_{2} \mathrm{O}\right]^{+} 273.290588$, observed 273.28983.<smiles>CN(CCO)CC(C)(C)C</smiles>

$N$-(2-hydroxyethyl)-N-methyl-1-dodecanylamine (2.6)

A solution of 2-(methylamino)ethanol $(1.37 \mathrm{~mL}, 17.0 \mathrm{mmol}), 1$-bromododecane (4.08 $\mathrm{mL}, 17.0 \mathrm{mmol})$, and anhydrous sodium carbonate $(1.93 \mathrm{~g}, 17.0 \mathrm{mmol})$ in anhydrous ethanol (34 mL) was heated to reflux. After $20 \mathrm{~h}$, the reaction suspension was cooled to room temperature and the precipitated solids were removed by filtration. The filtrate was concentrated to one-third volume by rotary evaporation and then transferred to a centrifuge vial and centrifuged to pellet precipitated solids. The supernatant was collected and evaporated by rotary evaporation. Bulb-to-bulb distillation (Kugelrohr, 190 ${ }^{\circ} \mathrm{C}$, ca. $\left.-0.986 \mathrm{~atm}\right)$ of the crude residue afforded $2.6(2.91 \mathrm{~g}, 70 \%)$ as a clear liquid. Spectral characterization of 2.6 gave values identical to those reported. ${ }^{168}{ }^{1} \mathrm{H}$ NMR (500 $\left.\mathrm{MHz}, \mathrm{CDCl}_{3}\right) \delta 0.88(\mathrm{t}, J=6.0 \mathrm{~Hz}, 3 \mathrm{H}) ; 1.25(\mathrm{~m}, 18 \mathrm{H}) ; 1.45(\mathrm{~m}, 2 \mathrm{H}), 2.22(\mathrm{~s}, 3 \mathrm{H}) ; 2.38(\mathrm{t}, J=$ $8.50 \mathrm{~Hz}, 2 \mathrm{H}) ; 2.50(\mathrm{t}, \mathrm{J}=4.25 \mathrm{~Hz}, 2 \mathrm{H}) ; 3.57(\mathrm{t}, \mathrm{J}=5.5 \mathrm{~Hz}, 2 \mathrm{H})$. 


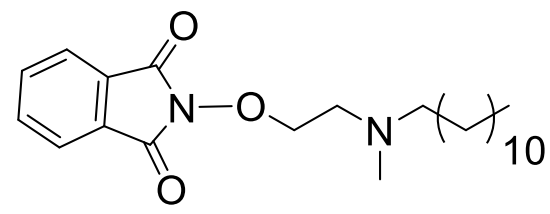

2-(2-(dodecyl(methyl)amino)ethoxy)isoindoline-1,3-dione (2.7)

To a solution of $2.6(2.91 \mathrm{~g}, 11.9 \mathrm{mmol})$ in THF $(50 \mathrm{~mL})$ at $0{ }^{\circ} \mathrm{C}$ were added $\mathrm{N}$ hydroxyphthalamide (2.14 g, $13.1 \mathrm{mmol}), \mathrm{PPh}_{3}(3.44 \mathrm{~g}, 13.1 \mathrm{mmol})$ and DIAD ( $2.6 \mathrm{~mL}, 13.1$ $\mathrm{mmol})$. The reaction mixture was warmed to $\mathrm{rt}$ and stirred $12 \mathrm{~h}$ before removing the solvents by rotary evaporation. To the yellow residue was added hexane $(60 \mathrm{~mL})$ and the resultant suspension was triturated and sonicated ( $\mathrm{rt}$ bath, $30 \mathrm{sec}$ intervals). The resultant fine solids were removed by filtration, and the filtrate was removed by rotary evaporation to afford crude 2.7 as an orange solid. The crude product was dissolved in ethyl acetate $(50 \mathrm{~mL})$ and the solution was washed with saturated $\mathrm{NaHCO}_{3}(3 \mathrm{X})$. The organic layer was dried $\left(\mathrm{Na}_{2} \mathrm{SO}_{4}\right)$ and then evaporated by rotary evaporation to yield 2.7 (4.53 g, 98\%) as a yellow, low melting semi-solid $\left(38.7-40.1{ }^{\circ} \mathrm{C}\right) ;{ }^{1} \mathrm{H} \mathrm{NMR}\left(500 \mathrm{MHz}, \mathrm{CDCl}_{3}\right)$ $\delta 0.87(\mathrm{t}, J=7.0 \mathrm{~Hz}, 3 \mathrm{H}) ; 1.24(\mathrm{~m}, 18 \mathrm{H}) ; 1.41(\mathrm{~m}, 2 \mathrm{H}) ; 2.29(\mathrm{~s}, 3 \mathrm{H}) ; 2.39(\mathrm{t}, J=7.8 \mathrm{~Hz}, 2 \mathrm{H})$; $2.83(\mathrm{t}, J=5.5 \mathrm{~Hz}, 2 \mathrm{H}) ; 4.32(\mathrm{t}, J=5.5 \mathrm{~Hz}, 2 \mathrm{H}) ; 7.74(\mathrm{~m}, 2 \mathrm{H}) ; 7.83(\mathrm{~m}, 2 \mathrm{H}) .{ }^{13} \mathrm{C}-\mathrm{NMR}(125$ $\left.\mathrm{MHz}, \mathrm{CDCl}_{3}\right) \delta 14.1 ; 21.9 ; 22.6 ; 26.9 ; 27.4 ; 29.3 ; 29.5-29.6(\mathrm{~m}) ; 31.9 ; 42.1 ; 55.4 ; 57.8 ; 75.6$; $123.4 ; 128.9 ; 134.4 ; 163.4$. 
$\mathrm{H}_{2} \mathrm{NO} \sim_{\mathrm{N}}^{\mathrm{I}} \mathrm{XT}_{10}^{+}$

$N$-[2-(Aminooxy)ethyl]- $N, N$-dimethyl-1-dodecylammonium iodide (QDA)

A glass pressure tube was charged with 2.7 (0.10 g, $1.79 \mathrm{mmol}), \mathrm{dry} \mathrm{CH}_{2} \mathrm{Cl}_{2}(5 \mathrm{~mL})$

and iodomethane $(1.53 \mathrm{~g}, 10.8 \mathrm{mmol})$. The tube was sealed and then immersed in an oil bath heated to $50^{\circ} \mathrm{C}$. After $12 \mathrm{~h}$, the reaction was cooled to rt. The tube was opened and the solvent was evaporated in a fume hood using a steady nitrogen stream. The resulting off-white solid 2.8 was used directly in the next step. ${ }^{1} \mathrm{H} \mathrm{NMR}\left(500 \mathrm{MHz}, \mathrm{CDCl}_{3}\right) \delta 0.85$ (t, $J=7.0 \mathrm{~Hz}, 3 \mathrm{H}) ; 1.24(\mathrm{~m}, 16 \mathrm{H}) ; 1.36(\mathrm{~m}, 2 \mathrm{H}) ; 1.84(\mathrm{~m}, 2 \mathrm{H}) ; 3.56(\mathrm{~s}, 6 \mathrm{H}) ; 3.77(\mathrm{~m}, 2 \mathrm{H}) ; 4.37$ $(\mathrm{t}, J=4.3 \mathrm{~Hz}, 2 \mathrm{H}) ; 4.75(\mathrm{t}, J=4.0 \mathrm{~Hz}, 2 \mathrm{H}) ; 7.80(\mathrm{~m}, 2 \mathrm{H}) ; 7.85(\mathrm{~m}, 2 \mathrm{H})$.

To a solution of $2.8(0.90 \mathrm{~g}, 1.70 \mathrm{mmol})$ in anhydrous ethanol $(9 \mathrm{~mL})$ was added hydrazine monohydrate $(0.43 \mathrm{~g} 8.49 \mathrm{mmol})$. The reaction mixture was stirred at $\mathrm{rt}$ for $12 \mathrm{~h}$ whereupon the solvent was removed by rotary evaporation. To the residue was added $\mathrm{CH}_{2} \mathrm{Cl}_{2}(20 \mathrm{~mL})$ and the resultant suspension was filtered. The filtrate was concentrated by rotary evaporation and toluene $(15 \mathrm{~mL})$ was added. The mixture was triturated with sonication ( $\mathrm{rt}$ bath, $30 \mathrm{sec}$ intervals) and the solids were collected and dried (vacuum) to afford QDA (0.45 g, 65\%); mp 85.9-88.9 ${ }^{\circ} \mathrm{C} ;{ }^{1} \mathrm{H}-\mathrm{NMR}\left(500 \mathrm{MHz}, \mathrm{CDCl}_{3}\right)$ $\delta 0.87(\mathrm{t}, J=7.0 \mathrm{~Hz}, 3 \mathrm{H}) ; 1.20-1.43(\mathrm{~m}, 18 \mathrm{H}) ; 1.75(\mathrm{~m}, 2 \mathrm{H}) ; 3.42(\mathrm{~s}, 6 \mathrm{H}) ; 3.58(\mathrm{~m}, 2 \mathrm{H}) ; 3.96$ $(\mathrm{t}, J=4.5 \mathrm{~Hz}, 2 \mathrm{H}) ; 4.16(\mathrm{~m}, 2 \mathrm{H}) ; 6.85$ (br. s, $2 \mathrm{H}) ;{ }^{13} \mathrm{C} \mathrm{NMR}\left(125 \mathrm{MHz}, \mathrm{CDCl}_{3}\right) \delta$ 14.1; 22.6; 22.9; 26.2; 29.2; 29.3; 29.35; 29.4; 29.6 (2 signals); 31.9; 52.1; 62.4; 66.0; 68.9; IR (DRIFT, $\mathrm{KBr}$ powder) $3270,2917,2848,1468,1172,956,900 \mathrm{~cm}^{-1}$; HRMS $\mathrm{m} / \mathrm{z}$ calcd $\left[\mathrm{C}_{16} \mathrm{H}_{37} \mathrm{~N}_{2} \mathrm{O}\right]^{+}$ 273.2906, observed 273.2903. 
<smiles>CC(=O)CC(C)(C)N(C)CCON</smiles>

$N$-[2-(Aminooxy)ethyl]-N-methyl- $N$-[( $\left.{ }^{13} \mathrm{C}^{2} \mathrm{H}_{3}\right)$-methyl]-1-dodecylammonium iodide (*QDA)

Using the procedure described for synthesis of QDA, amine $2.7(0.50 \mathrm{~g}, 1.29 \mathrm{mmol})$ was reacted with ${ }^{13} \mathrm{CD}_{3} \mathrm{l}(0.47 \mathrm{~g}, 3.21 \mathrm{mmol})$ and hydrazine monohydrate $(0.302 \mathrm{~g}$, $6.02 \mathrm{mmol})$ to give ${ }^{*} \mathrm{QDA}(0.37 \mathrm{~g}, 75 \%)$ as a white powder; $\mathrm{mp} 86.6-87.5^{\circ} \mathrm{C} ;{ }^{1} \mathrm{H}$ NMR (500 $\left.\mathrm{MHz}, \mathrm{CDCl}_{3}\right) \delta 0.86(\mathrm{t}, J=7.0 \mathrm{~Hz}, 3 \mathrm{H}) ; 1.14-1.43(\mathrm{~m}, 16 \mathrm{H}) ; 1.74(\mathrm{~m}, 2 \mathrm{H}) ; 3.41(\mathrm{~d}, J=3.5 \mathrm{~Hz}$, $3 \mathrm{H}) ; 3.58(\mathrm{~m}, 2 \mathrm{H}) ; 3.92(\mathrm{~m}, 2 \mathrm{H}) ; 4.16(\mathrm{~m}, 2 \mathrm{H}) ; 5.91$ (br s); ${ }^{13} \mathrm{C} \mathrm{NMR}\left(125 \mathrm{MHz}, \mathrm{CDCl}_{3}\right) \delta$ 14.3; 22.8; 23.1; 26.4; 29.4; 29.5; 29.6; 29.7; 29.8 (2 signals); 32.1; 51.6 (m); 52.2; 62.6; 66.0; 69.2; IR (DRIFT, KBr powder) 3278, 2913, 2850, 1468, 1173, 1048, 947 v(O-N), 720, 482, $418 \mathrm{~cm}^{-1} ;$ HRMS $\mathrm{m} / z$ calcd $\left[\mathrm{C}_{15}{ }^{13} \mathrm{C}^{2} \mathrm{H}_{3} \mathrm{H}_{34} \mathrm{~N}_{2} \mathrm{O}\right]^{+} 277.3119$, observed 277.3124 . 


\subsubsection{Mass spectrometry methods}

Samples were analyzed on a hybrid linear ion trap (LIT) FT-ICR mass spectrometer (LTQ-FT, Thermo Electron, Bremen, Germany) equipped with a TriVersa NanoMate ion source (Advion BioSciences, Ithaca, NY) with an " $A$ " electrospray chip (nozzle inner diameter $5.5 \mu \mathrm{m})$. The TriVersa NanoMate was operated in positive ion mode by applying $1.85 \mathrm{kV}$ with 0.1 psi head pressure. MS runs were recorded over a mass range from 150 to $1200 \mathrm{Da}$. Initially, low resolution LIT-MS scans were acquired for 0.75 minute to track the stability of the ion spray, after which high mass accuracy data were collected using the FT-ICR analyzer where MS scans were acquired for 8 min. The AGC (automatic gain control) maximum ion time was set to $10 \mathrm{~ms}$ and $500 \mathrm{~ms}$ for the LIT and ICR operations, respectively, using default ion count targets for both and 5 "microscans" (ICR transients) were accumulated before Fourier transformation to produce each saved spectrum; thus the cycle time for each transformed, saved spectrum was about $5 \mathrm{~s}$, the exact timing of which varied slightly with the AGC operations for each. The LTQ-FT was tuned and calibrated according to the manufacturer's default standard recommendations, which typically achieved better than 0.2 ppm mass accuracy at 400,000 resolving power $(\mathrm{m} / \mathrm{z}=$ 400).

FT-ICR mass spectra were exported as exact mass lists into a spreadsheet file using QualBrowser 2.0 SR2 (Thermo Electron, "Bremen" version for the LTQ-FT) by exporting all of the recorded peaks so that $\mathrm{s} / \mathrm{n}$ decisions can be made in downstream data processing. QDA/*QDA adducts were assigned based on their accurate mass by using an in-house software tool PREMISE (PRecaculated Exact Mass Isotopologue Search Engine) ${ }^{11}$ which 
was also manually validated. PREMISE is a simple algorithm that corrects small mass errors based on a selectable known peak (such as internal standard, or a known analyte), then matches observed with theoretical $\mathrm{m} / \mathrm{z}$ values within a selectable window that is typically set to $0.0008 \mathrm{Da}$ or smaller. The theoretical $\mathrm{m} / \mathrm{z}$ values of a large number of QDA-metabolite adducts were calculated and compiled in a reference table for the PREMISE assignment.

5.2.3 Extraction solvent determination, counterion comparison, and partition efficiency

One of three aminooxy reagents (QOA, QDA or *QDA) $9 \mu \mathrm{M}$ was added to a mixture of aldehyde/ketone standards (pyruvate, $\alpha$-ketoglutarate, $\alpha$-ketobutyrate, and glucose, $2 \mu \mathrm{M}$ each) in $5 \mathrm{mmM} \mathrm{pH} 6 \mathrm{NH}_{4} \mathrm{OAc}$ buffer in microcentrifuge tubes. The mixtures were sonicated $2 \mathrm{~h}$ at $\mathrm{rt}$ and placed in a dribath at $40{ }^{\circ} \mathrm{C}$ for $12 \mathrm{~h}$. The aqueous mixtures were then subjected to 5 equal volume extractions with either $\mathrm{CHCl}_{3}$, EtOAc, or $n$-butanol; each extraction was separately retained. The organic extractions were dried by vacuum centrifugation. The resultant residues were dissolved in $\mathrm{MeOH}$ with $0.1 \%$ formic acid and were detected by FT-ICR-MS after addition of a $200 \mathrm{nM}$ standard (QOAacetone- $d_{6}$ adduct) for normalization purposes. Ion counts were normalized to the standard and compared across the 5 extractions for the three solvents and the three aminooxy reagents. 


\subsubsection{Verification of linear instrument response}

As the automatic gain control of the FT-ICR-MS prevents the use of simple serial dilution of standards for signal intensity comparisons, the feature can be compensated for by adding a known standard at high concentration to all samples. A mixture of *QDA standard adducts (pyruvate, $\alpha$-ketoglutarate, $\alpha$-ketobutyrate, and glucose) were prepared in methanol and diluted to concentrations ranging from $25 \mu \mathrm{M}-25 \mathrm{pM}$. QOAacetone- $d_{6}$ adduct $(100 \mu \mathrm{M})$ was added as a standard for normalization and for use as a compensation for automatic gain control.

\subsubsection{Cell culture and extract preparation}

Human lung adenocarcinoma A549 cells were grown in $10-\mathrm{cm}$ plates with $8 \mathrm{~mL}$ RPMI 1640 medium (Sigma) supplemented with 2 mM glutamine, 0.2\% glucose, 10\% fetal bovine serum, $100 \mathrm{U} / \mathrm{mL}$ penicillin, and $0.1 \mathrm{mg} / \mathrm{mL}$ streptomycin at $37^{\circ} \mathrm{C}, 5 \% \mathrm{CO}_{2}$ and $95 \%$ humidity. When cells became $70 \%$ confluent, the medium was removed, and the cells were washed three times on ice each with $5 \mathrm{ml}$ cold 1X PBS (phosphate buffered saline) to remove medium components. $1 \mathrm{ml}$ of cold acetonitrile $\left(-20^{\circ} \mathrm{C}\right)$ was added to quench metabolism. Then, $0.75 \mathrm{~mL}$ of nanopure water was added before cells were scraped and collected into a $15 \mathrm{~mL}$ polypropylene conical centrifuge tube (Sarstedt, Newton, NC) containing three $3 \mathrm{~mm}$ diameter glass beads. These steps were then repeated to collect any remaining cells. Next, $1 \mathrm{~mL}$ HPLC-grade chloroform (Fisher Scientific) was added to the centrifuge tube containing the cell lysate. Extraction of polar metabolites via solvent 
partitioning was performed as described previously. ${ }^{169}$ The aqueous extract was lyophilized and stored at $-80^{\circ} \mathrm{C}$ prior to analysis.

\subsubsection{Cell extract derivatization}

$50 \mu \mathrm{L}$ of a 1:1 mixture of $100 \mu \mathrm{M}$ QDA and *QDA as solutions in nanopure water was added to a $1.5 \mathrm{~mL}$ microcentrifuge tube containing the aqueous extract of approximately one quarter of a $10 \mathrm{~cm}$ cell plate in $50 \mu \mathrm{L}$ of $10 \mathrm{mM} \mathrm{pH} 6 \mathrm{NH}_{4} \mathrm{OAc}$ buffer. The tube was briefly vortexed, sonicated for $1 \mathrm{hr}$ and placed in a $40{ }^{\circ} \mathrm{C}$ dry bath for 23 hours. Following reaction, the aqueous layer was extracted with $n$-butanol three times, and dried by vacuum centrifugation. The residue was dissolved in methanol with $0.1 \%$ v/v formic acid and analyzed by FT-ICR-MS as a 1:1 mixture with an external standard of $2 \mu \mathrm{M}$ QOA-acetone- $\mathrm{d}_{6}$ adduct. For each experiment, three replicate samples were made. A buffer blank consisting of $50 \mu \mathrm{L} 10 \mathrm{mM} \mathrm{pH} 6 \mathrm{NH}_{4} \mathrm{OAc}$ buffer and $50 \mu \mathrm{L}$ of the QDA/*QDA mixture was prepared and analyzed similarly to serve as a negative control. 


\subsubsection{Data analysis and statistics}

Each sample was acquired for nine minutes on FT-ICR-MS and the resultant spectra were averaged over all saved scans. Next, all peaks of a single (representative) acquisition were exported to excel. Any peaks with ion counts less than 1000 were deleted in order to eliminate noise, which typically appeared to be values around 200 counts or below. A theoretical " +4 " $\mathrm{m} / \mathrm{z}$ list was created by adding 4.02188 to all of the parent peaks. PREMISE software was then used to find matches between the original data and the +4 peak-list with an error range of $0.0009 \mathrm{~m} / \mathrm{z}$. This simplified list was then crosschecked manually against the negative control peak-list and any duplicate masses were deleted, except in cases where the intensity was significantly higher in the sample than the negative control $(>80 \%)$. Where multiple +4 peaks were matched, the peaks with ion counts most closely matching the parent $(n)$ peak were retained. Finally, any peaks identified as both $\mathrm{n}$ and $\mathrm{n}+4$ peaks were deleted. 


\subsubsection{Estimation of cellular suppression factor and quantification of pyruvate}

For an estimate of the "ion suppression" (loss of signal intensity) factor in cellular extracts, the ion counts of the parent ions (protonated) of QDA-pyruvate and QDA- ${ }^{13} C_{3^{-}}$ pyruvate were normalized by dividing by the ion counts of the external standard *QDApyruvate which was added at the same concentration to every sample. The normalized ion counts of $\mathrm{QDA}-{ }^{13} \mathrm{C}_{3}$-pyruvate were then plotted against the known concentrations of $2.5,5$, and $7.5 \mu \mathrm{M}$ in buffer conditions, and cell extract conditions, along with linear regressions. The quotient of the slopes of the two linear regressions gives a cellular extract ion suppression factor of 4.97 .

The concentration of endogenous pyruvate was calculated using the standard curve established by the internal standard $\mathrm{QDA}-{ }^{13} \mathrm{C}_{3}$ pyruvate. Specifically, the normalized ion counts for QDA-pyruvate were averaged across three nine-minute FT-ICRMS acquisitions, then over three replicate samples. Normalized ion counts of QDApyruvate from the blank (QDA added to buffer alone) were subtracted. This value was then entered as the $y$ value into the linear regression equation of Figure 2.10 and the $\mu \mathrm{M}$ concentration was calculated. This concentration indicated the amount of pyruvate (plus mass isomers) partitioned into the aqueous extract of $1 / 8^{\text {th }}$ of a $70 \%$ confluent $10 \mathrm{~cm}$ well plate (approx. 625,000 cells) dissolved in $100 \mu \mathrm{L}$ of methanol with $0.1 \%$ formic acid. 


\subsection{Experimental Procedures for Chapter 3}

5.3.1 Preparation of iron oxide NPs

5.3.2 Quantification of $\mathrm{Fe} 2+$ and total Fe for determination of NP composition

5.3.3 AMF heating properties

5.3.4 Syntheses of variably hydroxylated ammonium salts

5.3.5 NP coating with variably hydroxylated ammonium salts

5.3.6 Thermogravimetric analysis of NP-bound ammonium salts

5.3.7 Synthesis of FITC-CHO

5.3.8 Syntheses of aminooxy linkers

5.3.9 Fluorescence study of FITC-loaded NPs

5.3.10 Syntheses of doxorubicin analogs

5.3.11 Preparation of NP formulations of Dox analogs

5.3.12 UV-Vis detection of Dox-OH and Dox-2OH release by pulsed AMF

5.3.13 Toxicity of Dox-NPs in MCF-7 cells

5.3.14 Synthesis of QAO-2OH anthracene derivative

5.3.15 Dextran sulfate coating of NPs

5.3.16 Coating of dextran sulfate NPs with QAO-2OH anthracene derivative

5.3.17 Release of QAO-2OH anthracene derivative from dextran sulfate NPs 
5.3.18 $\mathrm{CS}_{2}$ and $\mathrm{CS}_{2} / \mathrm{CH}_{3}$ l coating of NPs

5.3.19 Synthesis of deuterated ammonium salt 3

5.3.20 Coating of $\mathrm{CS}_{2}$ NPs with 3

5.3.21 Synthesis of anthracen-9-ylmethanamine (4)

5.3.22 Preparation of thiocarbamate NPs 10

5.3.23 Elemental analysis

5.3.24 $\mathrm{CS}_{2}$ modified NPs with variably hydroxylated ammonium salts

5.3.25 Synthesis of greigite NPs

5.3.26 Greigite NP coating with QAO-2OH anthracene derivative 


\subsection{Experimental Procedures for Chapter 3}

\subsubsection{Preparation of iron oxide NPs}

$180 \mathrm{~mL}$ of $0.1 \mathrm{M} \mathrm{NaOH}(0.719 \mathrm{~g}, 18.0 \mathrm{mmol})$ in Millipore water was prepared and deoxygenated by bubbling with $\mathrm{N}_{2}$ for $1 \mathrm{~h} .5 .29 \mathrm{~mL}$ of $0.4 \mathrm{M}$ iron III chloride $(0.573 \mathrm{~g}, 2.12$ $\mathrm{mmol}$ ) and $5 \mathrm{~mL} \mathrm{0.2M}$ iron II chloride $(0.232 \mathrm{~g}, 1.17 \mathrm{mmol})$ were likewise prepared in deoxygenated Millipore water. $5 \mathrm{ml}$ each of the iron chloride solutions were combined and added dropwise to the aqueous base under rapid stirring. After 30 min the NPs were magnetically sedimented and the supernatant was aspirated under positive $\mathrm{N}_{2}$ pressure. The precipitate was washed with $40 \mathrm{~mL}$ degassed Millipore water ("water wash condition") and again magnetically separated for supernatant aspiration. NPs were transferred in a minimal amount of Millipore water to vials and freeze dried $(0.2417 \mathrm{~g}$, $89 \%)$.

For "acid washed" condition, NPs were prepared as above with the addition of $4 \mathrm{X}$ $40 \mathrm{~mL}$ washes with Millipore water followed by $1 \mathrm{X} 40 \mathrm{~mL}$ wash with $0.01 \mathrm{M}$ aqueous $\mathrm{HCl}$. For "multiple water wash" condition, NPs were simply washed $4 \mathrm{X}$ with $40 \mathrm{~mL}$ Millipore water.

\subsubsection{Quantification of $\mathrm{Fe}^{2+}$ and total Fe for determination of NP composition}

As outlined by Amonette and Templeton, ${ }^{105}$ the assay of Fe II and total Fe in iron oxide nanoparticles samples was accomplished by dissolving 1) 25 mg of "water washed" particles or 2) $27 \mathrm{mg}$ of "acid washed" particles in a preheated acid matrix consisting of $10 \%$ sulfuric acid (12 mL), hydrofluoric acid ( $1 \mathrm{~mL})$, and 10\% 1,10 phenanthroline in $95 \%$ 
ethanol (2 mL). After boiling for 30 minutes, boric acid solution (5\%, $10 \mathrm{~mL})$ ) and deionized water $(90 \mathrm{~mL})$ were added. An aliquot of each sample was reduced with $1 \%$ $\mathrm{NH}_{2} \mathrm{OH}$ in $1 \% \mathrm{Na}_{3}$-citrate $(10 \mathrm{~mL})$ to convert remaining Fe III to Fe II for a total Fe assay. Solutions were diluted in $1 \% \mathrm{Na}_{3}$-citrate and absorbances were detected on a Beckman Coulter DU 800 spectrophotometer. Absorbance at $510 \mathrm{~nm}$ was used to calculate total Fe and Fe II concentrations for each samples using equation 5 (ibid). Fe III was determined by the difference of Fe II from total Fe concentrations.

\subsubsection{AMF heating properties}

Solutions of NPs with concentrations of $0.2,1,5,25 \mathrm{mg} / \mathrm{mL}$ in Millipore water were prepared along with a control without NPs. Alginate (Tica-algin 613 sodium alginate: sodium phosphate, and calcium sulfate) was added such that each solution contained $3 \%$ w/v alginate. Each sample was exposed to AMF for $1 \mathrm{~h}$ at $201.4 \mathrm{~A}, 203 \mathrm{kHz}$ while temperature measurements were made at 15 minute intervals with a fiber optic temperature probe (Neoptix). 
5.3.4 Syntheses of variably hydroxylated ammonium salts

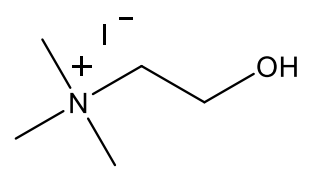

(2-hydroxyethyl)trimethylazanium iodide (10H)

$\mathrm{N}, \mathrm{N}$-dimethylethanolamine 4.99 mmols was added to a pressure vial as a $0.2 \mathrm{M}$ solution in $\mathrm{CH}_{2} \mathrm{Cl}_{2}$ and cooled to $0^{\circ} \mathrm{C}$. lodomethane was added (14.97 mmols) and the reaction was allowed to come to room temperature. After 12 hours at room temp, the solution was filtered of the white precipitated product. The precipitate was dried using a vacuum desiccator (1.61g, quantitative, mp. >260). ${ }^{1} \mathrm{H}$ NMR $\left(500 \mathrm{MHz}, \mathrm{D}_{2} \mathrm{O}\right) \delta 3.21(\mathrm{~s}, 9 \mathrm{H}$, $\left.\mathrm{N}\left(\mathrm{CH}_{3}\right)_{3}\right) ; 3.54\left(\mathrm{t}, 2 \mathrm{H}, \mathrm{J}=5.00 \mathrm{~Hz}, \mathrm{C}(1) \mathrm{H}_{2}\right) ; 4.08\left(\mathrm{~m}, 2 \mathrm{H}, \mathrm{C}(2) \mathrm{H}_{2}\right) .{ }^{13} \mathrm{C} \mathrm{NMR}\left(100 \mathrm{MHz}, \mathrm{D}_{2} \mathrm{O}\right)$ 3C $\delta 53.8 ; 55.5 ; 67.3 . \mathrm{m} / \mathrm{z}$ calcd $\left[\mathrm{C}_{5} \mathrm{H}_{14} \mathrm{NO}\right]^{+}$104.10699, observed 104.10693.

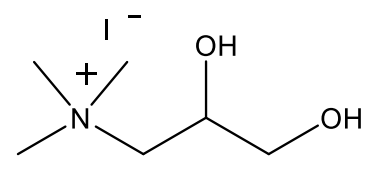

\section{(2,3-dihydroxypropyl)trimethylazanium iodide (1,2 diol)}

3-(dimethylamino)-1,2-propanediol (1.0 g, $8.4 \mathrm{mmols})$ was added to a pressure vial as a $1 \mathrm{M}$ solution in $\mathrm{CH}_{2} \mathrm{Cl}_{2}$. The vial was cooled to $0{ }^{\circ} \mathrm{C}$ and iodomethane $(3.6 \mathrm{~g}, 25.2$ mmols) was added. The sealed tube was capped and the reaction ran at room temperature for 10 hours with stirring. Post reaction, a white precipitate was filtered from solution. This retentate was rinsed with $\mathrm{CH}_{2} \mathrm{Cl}_{2}$ and dried (vacuum) affording the 1,2 diol $(2.077 \mathrm{~g}, 95 \%)$ as a white, crystalline solid, mp. $130.6-132.1^{\circ} \mathrm{C} .{ }^{1} \mathrm{H} \mathrm{NMR}(400 \mathrm{MHz}$, $\left.\mathrm{D}_{2} \mathrm{O}\right) \delta 3.29\left(\mathrm{~s}, 9 \mathrm{H}, \mathrm{N}\left(\mathrm{CH}_{3}\right)_{3}\right) ; 3.50\left(\mathrm{~m}, 2 \mathrm{H} \mathrm{C}(1) \mathrm{H}, \mathrm{C}(1) \mathrm{H}^{\prime}\right) ; 3.64\left(\mathrm{~m}, 2 \mathrm{H}, \mathrm{C}(3) \mathrm{H}_{2}\right) ; 4.35(\mathrm{~m}, 1 \mathrm{H}$, 
$\mathrm{C}(2) \mathrm{H}) .{ }^{13} \mathrm{C} \mathrm{NMR}\left(125 \mathrm{MHz}, \mathrm{D}_{2} \mathrm{O}\right) 4 \mathrm{C} \delta 54.29 ; 63.74 ; 66.49 ; 68.30 . \mathrm{m} / z$ calcd $\left[\mathrm{C}_{6} \mathrm{H}_{16} \mathrm{NO}_{2}\right]^{+}$ 134.117555, observed 134.11748.

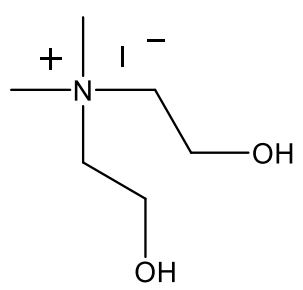

bis(2-hydroxyethyl)dimethylazanium iodide (2OH)

$\mathrm{N}$-methyl diethanolamine $(1.0 \mathrm{~g}, 8.4 \mathrm{mmols})$ was added to a pressure vial as a $1 \mathrm{M}$ solution in $\mathrm{CH}_{2} \mathrm{Cl}_{2}$. lodomethane $(5.9 \mathrm{~g}, 42.0 \mathrm{mmol}$ ) was added and the sealed tube was heated in an oil bath at $50^{\circ} \mathrm{C}$ for 10 hours with magnetic stirring. Post reaction, the upper clear solution was decanted and the yellow oil was washed with $\mathrm{CH}_{2} \mathrm{Cl}_{2}(15 \mathrm{~mL})$. Acetonitrile was used to dissolve the yellow oil, the solvent was removed by rotary evaporation and drying (vacuum) afforded $2 \mathrm{OH}$ as a hygroscopic orange solid, mp. 76.4$78.9^{\circ} \mathrm{C}(2.55 \mathrm{~g}, 119 \%) .{ }^{1} \mathrm{H}$ NMR $\left(500 \mathrm{MHz}, \mathrm{D}_{2} \mathrm{O}\right) \delta 3.25\left(\mathrm{~s}, 6 \mathrm{H}, \mathrm{N}\left(\mathrm{CH}_{3}\right)_{2}\right) ; 3.62(\mathrm{t}, 4 \mathrm{H}, \mathrm{J}=5.00$ $\left.\mathrm{Hz}, \mathrm{C}(1) \mathrm{H}_{2}\right) ; 4.09\left(\mathrm{~m}, 4 \mathrm{H}, \mathrm{C}(2) \mathrm{H}_{2}\right) .{ }^{13} \mathrm{C}$ NMR $\left(100 \mathrm{MHz}, \mathrm{D}_{2} \mathrm{O}\right) 3 \mathrm{C} \delta 52.2 ; 55.3 ; 66.9 . \mathrm{m} / \mathrm{z}$ calcd $\left[\mathrm{C}_{6} \mathrm{H}_{16} \mathrm{NO}_{2}\right]^{+}$134.117555, observed 134.11749. 


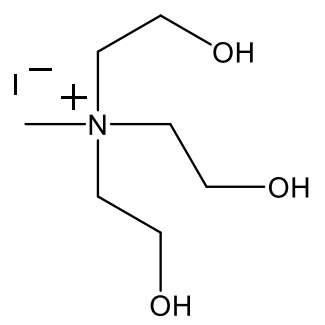

tris(2-hydroxyethyl)(methyl)azanium iodide (3OH)

Triethanolamine (1.0 g, $6.7 \mathrm{mmols})$ was added to a pressure vial as a $1 \mathrm{M}$ solution in $\mathrm{CH}_{2} \mathrm{Cl}_{2}$. lodomethane $(2.85 \mathrm{~g}, 20.1 \mathrm{mmol})$ was added and the sealed tube was heated in an oil bath at $50^{\circ} \mathrm{C}$ for 6 hours with magnetic stirring. Post reaction, the upper clear solution was decanted and the amber oil was washed with $\mathrm{CH}_{2} \mathrm{Cl}_{2}(8 \mathrm{~mL})$. Acetonitrile was used to dissolve the oil, the solvent was removed by rotary evaporation and drying (vacuum) afforded $3 \mathrm{OH}$ as an orange semisolid (2.55 g, 119\%). ${ }^{1} \mathrm{H} \mathrm{NMR}\left(400 \mathrm{MHz}, \mathrm{D}_{2} \mathrm{O}\right)$ $\delta 3.28\left(\mathrm{~s}, 3 \mathrm{H}, \mathrm{N}\left(\mathrm{CH}_{3}\right)\right) ; 3.70\left(\mathrm{t}, 6 \mathrm{H}, \mathrm{J}=5.00 \mathrm{~Hz}, \mathrm{C}(1) \mathrm{H}_{2}\right) ; 4.09\left(\mathrm{~m}, 6 \mathrm{H}, \mathrm{C}(2) \mathrm{H}_{2}\right) .{ }^{13} \mathrm{C} \mathrm{NMR}(100$ $\left.\mathrm{MHz}, \mathrm{D}_{2} \mathrm{O}\right) 3 \mathrm{C} \delta 50.0 ; 55.1 ; 64.4 . \mathrm{m} / \mathrm{z}$ calcd $\left[\mathrm{C}_{7} \mathrm{H}_{18} \mathrm{NO}_{3}\right]^{+}$164.12812, observed 164.12804.

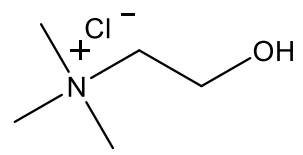

(2-hydroxyethyl)trimethylazanium chloride (10H)

A Dowex ion exchange resin (10g, 200-400 mesh, chloride form) was hydrated and packed in water; the eluent was then gradually switched to methanol. The iodide form of $10 \mathrm{H}(0.21 \mathrm{~g}, 0.89 \mathrm{mmols})$ was passed through the resin using gravity elution with methanol. The product, a white solid $(0.143 \mathrm{~g}, 115 \%)$, was verified as the chloride salt using the silver nitrate method as well as by mass spectrometry. ${ }^{1} \mathrm{H} N M R\left(500 \mathrm{MHz}, \mathrm{D}_{2} \mathrm{O}\right)$ 
$\delta 3.21\left(\mathrm{~s}, 9 \mathrm{H}, \mathrm{N}\left(\mathrm{CH}_{3}\right)_{3}\right) ; 3.53\left(\mathrm{t}, 2 \mathrm{H}, \mathrm{J}=5.00 \mathrm{~Hz}, \mathrm{C}(1) \mathrm{H}_{2}\right) ; 4.07\left(\mathrm{~m}, 2 \mathrm{H}, \mathrm{C}(2) \mathrm{H}_{2}\right) .{ }^{13} \mathrm{C} \mathrm{NMR}(125$ $\left.\mathrm{MHz}, \mathrm{D}_{2} \mathrm{O}\right) 3 \mathrm{C} \delta 54.0 ; 55.7 ; 67.5 . \mathrm{m} / \mathrm{z}$ calcd $\left[\mathrm{C}_{5} \mathrm{H}_{14} \mathrm{NO}^{+}\right.$104.10699, observed 104.10693. Dimer (chloride counterion) $\mathrm{m} / \mathrm{z}$ calcd 243.182833 , observed 243.18357 , no iodide dimer observed.

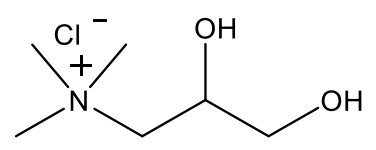

(2,3-dihydroxypropyl)trimethylazanium chloride (1,2 diol)

The iodo form of the $1,2 \mathrm{diol}(0.21 \mathrm{~g}, 0.79 \mathrm{mmols})$ was passed through an ion exchange resin prepared as above using gravity elution with methanol. The product, a pale yellow semi-solid $(0.142 \mathrm{~g}, 106 \%)$, was verified as the chloride salt using mass spectrometry. ${ }^{1} \mathrm{H} N M R\left(500 \mathrm{MHz}, \mathrm{D}_{2} \mathrm{O}\right) \delta 3.24\left(\mathrm{~s}, 9 \mathrm{H}, \mathrm{N}\left(\mathrm{CH}_{3}\right)_{3}\right) ; 3.46\left(\mathrm{~m}, 2 \mathrm{H} \mathrm{C}(1) \mathrm{H}, \mathrm{C}(1) \mathrm{H}^{\prime}\right)$; $3.60\left(\mathrm{~d}, 2 \mathrm{H}, \mathrm{J}=5.00 \mathrm{~Hz}, \mathrm{C}(3) \mathrm{H}_{2}\right) ; 4.30(\mathrm{~m}, 1 \mathrm{H}, \mathrm{C}(2) \mathrm{H}) .{ }^{13} \mathrm{C} N M R\left(125 \mathrm{MHz}, \mathrm{D}_{2} \mathrm{O}\right)$ 4C $\delta$ 68.3; 66.5; 63.7, 54.3. $\mathrm{m} / \mathrm{z}$ calcd $\left[\mathrm{C}_{6} \mathrm{H}_{16} \mathrm{NO}_{2}\right]^{+}$134.117555, observed 134.11748. Dimer (chloride counterion) $\mathrm{m} / \mathrm{z}$ calcd 303.203963 , observed 303.20481 , no iodide dimer observed. 


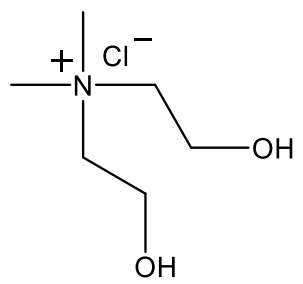

bis(2-hydroxyethyl)dimethylazanium chloride (2OH)

The iodo form of $2 \mathrm{OH}(0.21 \mathrm{~g}, 0.82 \mathrm{mmols})$ was passed through an ion exchange column prepared as above using gravity elution with methanol. The product, a transparent solid $\left(0.146 \mathrm{~g}, 105 \%, 104^{\circ} \mathrm{C}(\mathrm{d})\right)$, was verified as the chloride salt using mass spectrometry. ${ }^{1} \mathrm{H}$ NMR $\left(500 \mathrm{MHz}, \mathrm{D}_{2} \mathrm{O}\right) \delta 3.23\left(\mathrm{~s}, 6 \mathrm{H}, \mathrm{N}\left(\mathrm{CH}_{3}\right)_{2}\right) ; 3.60(\mathrm{t}, 4 \mathrm{H}, \mathrm{J}=5.00 \mathrm{~Hz}$, $\left.\mathrm{C}(1) \mathrm{H}_{2}\right) ; 4.08\left(\mathrm{~m}, 4 \mathrm{H}, \mathrm{C}(2) \mathrm{H}_{2}\right) .{ }^{13} \mathrm{C} \mathrm{NMR}\left(100 \mathrm{MHz}, \mathrm{D}_{2} \mathrm{O}\right) 3 \mathrm{C} \delta 52.3 ; 55.5 ; 66.3 . \mathrm{m} / \mathrm{z}$ calcd $\left[\mathrm{C}_{6} \mathrm{H}_{16} \mathrm{NO}_{2}\right]^{+}$134.117555, observed 134.11748. Dimer (chloride counterion) $\mathrm{m} / \mathrm{z}$ calcd 303.203963, observed 303.20479, no iodide dimer observed.

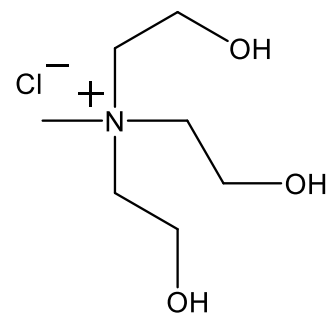

tris(2-hydroxyethyl)(methyl)azanium chloride (3OH)

The iodo form of $3 \mathrm{OH}(0.21 \mathrm{~g}, 0.72 \mathrm{mmols})$ was passed through an ion exchange column prepared as above using gravity elution with methanol. The product, a transparent hygroscopic solid $(0.148 \mathrm{~g}, 104 \%)$, was verified as the chloride salt using mass spectrometry. ${ }^{1} \mathrm{H}$ NMR $\left(500 \mathrm{MHz}, \mathrm{D}_{2} \mathrm{O}\right) \delta 3.26\left(\mathrm{~s}, 3 \mathrm{H}, \mathrm{N}\left(\mathrm{CH}_{3}\right)\right) ; 3.69(\mathrm{t}, 6 \mathrm{H}, \mathrm{J}=5.25 \mathrm{~Hz}$, 
$\left.\mathrm{C}(1) \mathrm{H}_{2}\right) ; 4.08\left(\mathrm{~m}, 6 \mathrm{H}, \mathrm{C}(2) \mathrm{H}_{2}\right) .{ }^{13} \mathrm{C} \mathrm{NMR}\left(125 \mathrm{MHz}, \mathrm{D}_{2} \mathrm{O}\right) 3 \mathrm{C} \delta 50.1 ; 55.3 ; 64.5 . \mathrm{m} / \mathrm{z}$ calcd $\left[\mathrm{C}_{7} \mathrm{H}_{18} \mathrm{NO}_{3}\right]^{+}$164.12812, observed 164.12818. Dimer (chloride counterion) $\mathrm{m} / \mathrm{z}$ calcd 363.225093, observed 363.22728, no iodide dimer observed.

5.3.5 NP coating with variably hydroxylated ammonium salts

$3 \mathrm{M}$ solutions in methanol of each ammonium salt (or amine free base) were prepared and added to microcentrifuge tubes containing $\approx 10 \mathrm{mg}$ NPs such that there were $10 \mathrm{mmol}$ ammonium salt per g NP. The microcentrifuge tubes were briefly vortexed and sonicated $(\mathrm{rt}, 1 \mathrm{~h})$ followed by rotating on an inverter at $\mathrm{rt}$ for $3 \mathrm{~h}$. NPs were pelleted by centrifugation (16000 rcf, $20 \mathrm{~min}$ ) and the supernatant was removed. The NPs were then washed with water $(3 \times 0.5 \mathrm{~mL})$ with intervening pelleting by centrifugation. The pellets were then freeze dried.

\subsubsection{Thermogravimetric analysis of NP-bound ammonium salts}

TGA was performed on a TA Instruments Hi-Res 2950 Thermogravimetric Analyzer. Approximately $6 \mathrm{mg}$ of each lyophilyzed NP sample was loaded into a platinum sample pan for analysis. After an initial equilibration phase at $35^{\circ} \mathrm{C}$, the temperature was increased $20^{\circ} \mathrm{C} / \mathrm{min}$ up to $700^{\circ} \mathrm{C}$ under nitrogen gas. Triplicate samples were analyzed. 


\subsubsection{Synthesis of FITC-CHO}

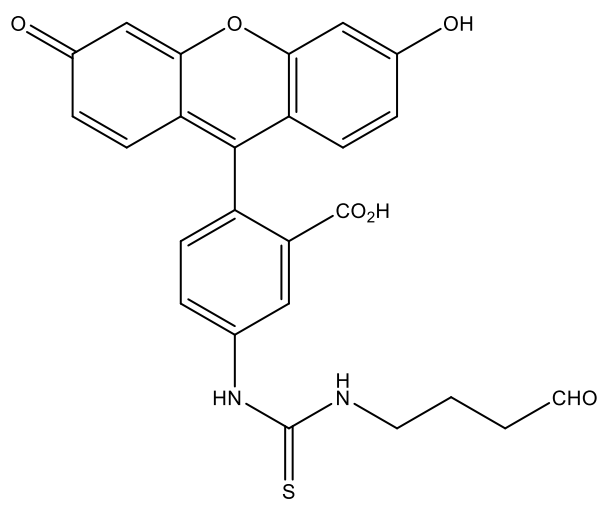

To a solution of fluorescein isothiocyanate ( $80 \%$ isomer $1,1.28 \mathrm{~g}, 3.28 \mathrm{mmol}$ ) in DMF (10 mL) was added 4-aminobutyraldehyde diethyl acetal $(0.771 \mathrm{~g}, 3.94 \mathrm{mmol})$ via syringe. The mixture was stirred $4 \mathrm{~h}$ at $\mathrm{rt}$. The reaction solvent was diluted with $100 \mathrm{~mL}$ dilute aq. $\mathrm{HCl}(\mathrm{pH} 4)$ and extracted with diethyl ether $(8 \times 100 \mathrm{~mL})$. The organic phase was dried over sodium sulfate and evaporated by rotary evaporation. The product was passed through a silica gel plug $\left(\mathrm{CH}_{2} \mathrm{Cl}_{2}: \mathrm{MeOH}, 1: 0-9: 1\right)$ and was used in the next step without further purification.

To the acetal was added $30 \% \mathrm{v} / \mathrm{v}$ aqueous acetic acid $(150 \mathrm{~mL})$ and stirred $15 \mathrm{~h}$ at rt. The solvent was removed as an azeotrope with toluene to yield FITC-CHO as an orange solid (0.550 g, 55\% 2 steps). ${ }^{1} \mathrm{H}$ NMR (400 MHz, dmso) major isomer $\delta=10.11$ (br s, 2H); $8.12(\mathrm{~s}, 1 \mathrm{H}) ; 7.84(\mathrm{~m}, 1 \mathrm{H}) ; 7.26-7.12(\mathrm{~m}, 2 \mathrm{H}) ; 6.66(\mathrm{~s}, 2 \mathrm{H}) ; 6.58(\mathrm{~m}, 4 \mathrm{H}) ; 5.76(\mathrm{br} \mathrm{s}, 1 \mathrm{H})$; $3.78(\mathrm{~m}, 1 \mathrm{H}) ; 3.61(\mathrm{~m}, 2 \mathrm{H}) ; 2.30(\mathrm{~s}, 1 \mathrm{H}) ; 2.09(\mathrm{br}, 2 \mathrm{H}) ; 1.89(\mathrm{br}, 2 \mathrm{H})$. HRMS m/z calcd $\left[\mathrm{C}_{6} \mathrm{H}_{16} \mathrm{NO}_{2}\right]^{-}$475.0969, observed 475.09688. 


\subsubsection{Syntheses of aminooxy linkers}

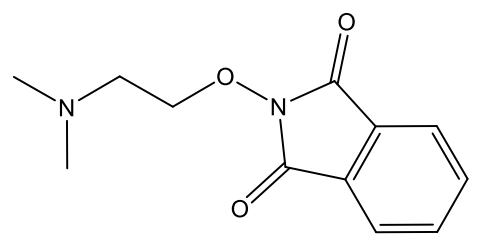

2-[2-(dimethylamino)ethoxy]-2,3-dihydro-1H-isoindole-1,3-dione

To a solution of triphenylphosphine $(5.3 \mathrm{~g}, 20.2 \mathrm{mmol})$ and $N$-hydroxyphthalimide (3.29 g, $20.2 \mathrm{mmol})$ in anhydrous THF $(50 \mathrm{~mL})$ at $0{ }^{\circ} \mathrm{C}$ was added $N, N$ dimethyl ethanolamine ( $1.50 \mathrm{~g}, 16.80 \mathrm{mmol})$. After stirring $20 \mathrm{~min}$, DIAD (4.08 g, $20.18 \mathrm{mmol}$ ) was added dropwise. The reaction mixture was then allowed to warm to room temperature. After $12 \mathrm{~h}$, the solvent was removed by rotary evaporation. EtOAc (300 mL) was added to dissolve the residue and the organic phase was washed with saturated aq. $\mathrm{NaHCO}_{3}(3$ $X 200 \mathrm{~mL})$, and brine $(3 \mathrm{X} 200 \mathrm{~mL})$. The EtOAc was dried $\left(\mathrm{Na}_{2} \mathrm{SO}_{4}\right)$, and concentrated by rotary evaporation. The residue was redissolved in EtOAc $(250 \mathrm{~mL})$ and extracted $3 \mathrm{X}$ with $50 \mathrm{~mL}$ portions of $5 \% \mathrm{v} / \mathrm{v}$ aqueous $\mathrm{HCl}$ and washed with $\mathrm{Et}_{2} \mathrm{O}$. Saturated $\mathrm{NaHCO}_{3}$ was then added until the $\mathrm{pH}$ registered slightly basic and the aqueous phase was extracted with $\mathrm{CHCl}_{3}(3 \times 100 \mathrm{~mL})$. The $\mathrm{CHCl}_{3}$ was evaporated to yield a yellow solid $(1.56 \mathrm{~g}, 40 \%)$. ${ }^{1} \mathrm{H}$ NMR $\left(500 \mathrm{MHz}, \mathrm{DMSO}-\mathrm{d}_{6}\right) \delta 7.86(\mathrm{~s}, 4 \mathrm{H}) ; 4.21(\mathrm{~s}, 2 \mathrm{H}) ; 2.61(\mathrm{~s}, 2 \mathrm{H}) ; 2.18(\mathrm{~s}, 6 \mathrm{H})$. 


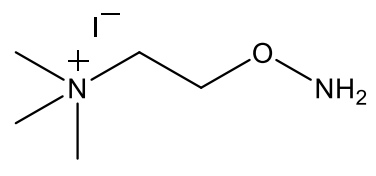

O-[2-(trimethylazaniumyl)ethyl]hydroxylamine iodide (QAO)

To the phthalimide protected amine shown above $(0.78 \mathrm{~g}, 3.33 \mathrm{mmol})$ in $\mathrm{CH}_{2} \mathrm{Cl}_{2}$ $(10 \mathrm{~mL})$ in a pressure vial was added iodomethane $(11.4 \mathrm{~g}, 79.7 \mathrm{mmol})$. The reaction was sealed and heated at $50{ }^{\circ} \mathrm{C}$ for $12 \mathrm{~h}$. After cooling, the solvent and excess iodomethane were evaporated under $\mathrm{N}_{2}$ stream in a fume hood. The isolated white solids were used directly in the next step. ${ }^{1} \mathrm{H}$ NMR $(400 \mathrm{MHz}$, DMSO-d $)$ ) $7.90(\mathrm{~s}, 4 \mathrm{H}) ; 4.67(\mathrm{~s}, 2 \mathrm{H}) ; 3.79$ (s, $2 \mathrm{H}) ; 3.23(\mathrm{~s}, 9 \mathrm{H})$.

To the iodo salt $(1.46 \mathrm{~g}, 5.77 \mathrm{mmol})$ in ethanol $(20 \mathrm{~mL})$ was added hydrazine monohydrate $(0.92 \mathrm{~g}, 17.8 \mathrm{mmol})$. The reaction was stirred $4 \mathrm{~h}$ at $\mathrm{rt}$ and the solvent was removed by rotary evaporation. The resultant residue was triturated in hot acetonitrile, filtered, and the filtrated was evaporated to yield a yellow amorphous solid (QAO). ${ }^{1} \mathrm{H}$ NMR (400 MHz, D $\left.{ }_{2} \mathrm{O}\right) \delta 4.14(\mathrm{~m}, 2 \mathrm{H}) ; 3.63(\mathrm{t}, J=4.6 \mathrm{~Hz}, 2 \mathrm{H}), 3.19(\mathrm{~s}, 9 \mathrm{H})$. 


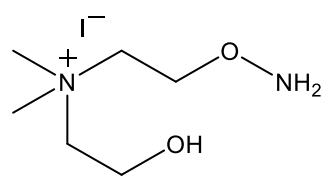

[2-(aminooxy)ethyl](2-hydroxyethyl)dimethylazanium iodide (QAO-1OH)

QAO-1OH was prepared by my colleague, Dr. Xuan Huang as reported in Tetrahedron Letters 2010, 51 (13), 1727-1729.

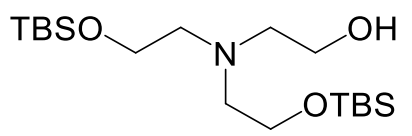

Synthesis of $\mathbf{3 . 1}$

To a solution of triethanolamine $(56.7 \mathrm{~g}, 380.0 \mathrm{mmol})$ and triethylamine $(38.4 \mathrm{~g}$, $380.0 \mathrm{mmol})$ at $0{ }^{\circ} \mathrm{C}$, a solution of tert-butyldimethylsilyl chloride $(68.7 \mathrm{~g}, 455.9 \mathrm{mmol})$ in $\mathrm{CH}_{2} \mathrm{Cl}_{2}(40 \mathrm{~mL})$ was added via cannula. The reaction mixture was stirred $1 \mathrm{~h}$ at $0{ }^{\circ} \mathrm{C}$ then was allowed to warm to $\mathrm{rt}$. After $12 \mathrm{~h}$ the reaction solvent was removed by rotary evaporation. The crude residue was purified by flash chromatography using 2:1 hexanes:ethyl acetate as eluant. The product was a clear oil (12.7g, 13\%). ${ }^{1} \mathrm{H}$ NMR (CDCl 3 , $400 \mathrm{MHz}) \delta 3.66(\mathrm{~m}, 4 \mathrm{H}), 3.52(\mathrm{~m}, 2 \mathrm{H}), 6.14(\mathrm{~m}, 6 \mathrm{H}), 0.89(\mathrm{~s}, 18 \mathrm{H}), 0.05(\mathrm{~s}, 12 \mathrm{H})$. 
TBSO<smiles>[SbH2]OCCOc1ccccc1</smiles>

Synthesis of $\mathbf{3 . 2}$

To a solution of triphenylphosphine $(3.055 \mathrm{~g}, 11.649 \mathrm{mmol})$ and $\mathrm{N}$ hydroxyphthalimide $(1.90 \mathrm{~g}, 11.65 \mathrm{mmol})$ in anhydrous THF $(50 \mathrm{~mL})$ at $0{ }^{\circ} \mathrm{C}$ was added dropwise the bis- $t$-butylated amine $3.1(4.0 \mathrm{~g}, 10.59 \mathrm{mmol})$. After stirring $30 \mathrm{~min}$, diisopropyl azodicarboxylate (DIAD) $(2.3 \mathrm{~g}, 11.6 \mathrm{mmol})$ was added dropwise via syringe. The reaction mixture was then allowed to warm to room temperature. After $12 \mathrm{~h}$, the solvent was removed by rotary evaporation. Diethyl ether $(150 \mathrm{~mL})$ was added to dissolve the orange residue and the organic phase was washed with successive washings of saturated aq. $\mathrm{NaHCO}_{3}(3 \times 100 \mathrm{~mL})$, and brine $(100 \mathrm{~mL})$. The ether was dried $\left(\mathrm{Na}_{2} \mathrm{SO}_{4}\right)$, filtered, and concentrated to a viscous yellow-orange semisolid by rotary evaporation. The product was purified by silica gel chromatography eluting in $4: 1 \mathrm{CH}_{2} \mathrm{Cl}_{2}$ /EtOAc. $\mathrm{Rf}$ 0.59 in 2:1 hexanes:EtOAc. The product is a yellow oil $(4.5 \mathrm{~g}, 81 \%) .{ }^{1} \mathrm{H}$ NMR $\left(\mathrm{CDCl}_{3}, 400\right.$ $\mathrm{MHz}) \delta 7.83(\mathrm{~m}, 2 \mathrm{H}), 7.74(\mathrm{~m}, 2 \mathrm{H}), 4.27(\mathrm{t}, J=6.0 \mathrm{~Hz}, 2 \mathrm{H}), 3.67(\mathrm{t}, J=6.4 \mathrm{~Hz}, 4 \mathrm{H}) 3.06(\mathrm{t}, J$ $=6 \mathrm{~Hz}, 2 \mathrm{H}) ; 2.77(\mathrm{t}, \mathrm{J}=6.6 \mathrm{~Hz}, 4 \mathrm{H}), 0.86(\mathrm{~s}, 18 \mathrm{H}), 0.04(\mathrm{~s}, 12 \mathrm{H})$. 


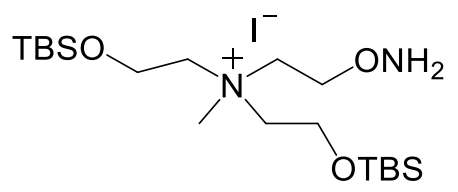

Synthesis of $\mathbf{3 . 3}$

The phthalimide protected amine $3.2(4.5 \mathrm{~g}, 8.5 \mathrm{mmol})$ was dissolved in anhydrous $\mathrm{CH}_{2} \mathrm{Cl}_{2}(20 \mathrm{~mL})$ and placed in a pressure vial. lodomethane $(6.1 \mathrm{~g}, 42.8 \mathrm{mmol})$ was added dropwise. The vial was sealed and warmed to $50{ }^{\circ} \mathrm{C}$. After $5 \mathrm{~h}$, the vial was cooled, opened, and the solvent was evaporated under reduced pressure in a fume hood to afford the crude ammonium iodide 3.3 as a light yellow solid $(5.3 \mathrm{~g}, 92 \%)$ which was used directly in the next step. ${ }^{1} \mathrm{H} N M R\left(\mathrm{CDCl}_{3}, 400 \mathrm{MHz}\right) \delta 7.82(\mathrm{~m}, 4 \mathrm{H}), 4.73(\mathrm{~m}, 2 \mathrm{H}), 4.41(\mathrm{~m}, 2 \mathrm{H}), 4.15$ $(\mathrm{m}, 8 \mathrm{H}) 3.64(\mathrm{~s}, 3 \mathrm{H}) ; 0.88(\mathrm{~s}, 18 \mathrm{H}), 0.11(\mathrm{~s}, 12 \mathrm{H})$.

The crude iodo salt 3.3 (15.2 g, $22.8 \mathrm{mmol})$ was dissolved in 95\% EtOH (115 mL). Hydrazine monohydrate $(4.6 \mathrm{~g}, 91.3 \mathrm{mmol})$ was added. The reaction mixture was stirred $24 \mathrm{~h}$ at room temperature. The solvent was removed by rotary evaporation and the residue was dissolved in $\mathrm{CH}_{2} \mathrm{Cl}_{2}(10 \mathrm{~mL})$. The white precipitate was filtered over a Buchner funnel and the $\mathrm{CH}_{2} \mathrm{Cl}_{2}$ filtrate was condensed by rotary evaporation. A pale yellow solid was isolated (12.0 g, 98\%, $\mathrm{Rf}_{\mathrm{f}}$ 0.61in 9:1 $\left.\mathrm{MeOH} / \mathrm{CH}_{2} \mathrm{Cl}_{2}\right) .{ }^{1} \mathrm{H} \mathrm{NMR}\left(\mathrm{CDCl}_{3}, 400 \mathrm{MHz}\right) \delta 5.90$ (bs, 2H), $4.17(\mathrm{~m}, 2 \mathrm{H}), 4.09(\mathrm{~m}, 4 \mathrm{H}), 4.02(\mathrm{t}, \mathrm{J}=4.2 \mathrm{~Hz}, 2 \mathrm{H}), 3.97(\mathrm{~m}, 4 \mathrm{H}), 3.47(\mathrm{~s}, 3 \mathrm{H}), 0.88$ (s, 18H), 0.09 (s, 12H). 


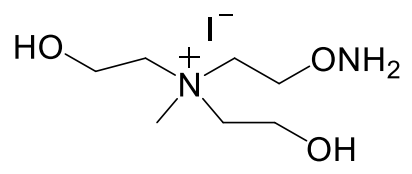

[2-(aminooxy)ethyl]bis(2-hydroxyethyl)methylazanium iodide (QAO-2OH)

The silylated aminooxy salt $3.3(2.971 \mathrm{~g}, 5.55 \mathrm{mmol})$ was stirred in anhydrous $\mathrm{CH}_{2} \mathrm{Cl}_{2}$ at $0{ }^{\circ} \mathrm{C}$ for 5 minutes, tetrabutylammonium fluoride $(11.1 \mathrm{~mL}$ of $1 \mathrm{M}$ in THF) was added dropwise to the reaction mixture. The reaction was stirred at room temperature for $3 \mathrm{~h}$. The solvent was removed by rotary evaporation. The resulting pale yellow solid was dissolved in water and purified by C18 reverse phase flash chromatography eluting in 97:3 water:acetonitrile. The aqueous solvents were removed by rotary evaporation and vacuum drying. The product was isolated as colorless oil $(0.61 \mathrm{~g}, 36 \%) .{ }^{1} \mathrm{H} N M R\left(\mathrm{D}_{2} \mathrm{O}, 400\right.$ MHz) $\delta 4.17(\mathrm{~m}, 2 \mathrm{H}), 4.07(\mathrm{~m}, 4 \mathrm{H}), 3.80(\mathrm{t}, J=4.8 \mathrm{~Hz} 2 \mathrm{H}), 3.67(\mathrm{t}, J=5.0 \mathrm{~Hz}, 4 \mathrm{H}), 3.25(\mathrm{~s}$, 3H). ${ }^{13} \mathrm{C}$ NMR $\left(125 \mathrm{MHz} \mathrm{D}_{2} \mathrm{O}\right) \delta 68.5,64.4,61.6,55.2,49.9$. 


\subsubsection{Fluorescence study of FITC-loaded NPs}

Aqueous solutions (0.1 M) of QAO, QAO- $10 \mathrm{H}$, and $\mathrm{QAO}-2 \mathrm{OH}$ compound were added to suspensions of iron oxide nanoparticles ( $3 \mathrm{mg}$ each) in vials ( $5 \mu$ moles of aminooxy compound/mg of nanoparticles). The vials were briefly sonicated (15 minutes) and then vortex mixed for 45 minutes at room temperature. The resultant QAO-coated NPs were magnetically separated from the supernatant solution, washed with water to remove unbound QAO salts, and freeze dried.

To a suspension of the QAO-NPs ( $3 \mathrm{mg}$ ) in methanol at room temperature was added a solution of FITC-CHO in methanol $(0.01 \mathrm{M}, 50 \mu \mathrm{L} / \mathrm{mg}$ of NPs). The reaction mixture was vortex mixed 2 hours at room temperature. The particles then were magnetically separated, the supernatant was removed and analyzed. The particles were washed twice with methanol ( $3 \mathrm{X}$ reaction volume) and the supernatants collected each time and analyzed. Water was then added (50 $\mathrm{LL} / \mathrm{mg} \mathrm{NPs})$, an aliquot was collected for analysis, and the aqueous suspensions then were placed in an Easy Heat LI alternating electromagnetic field at 201.4A and $203 \mathrm{kHz}$ for 30 minutes. After AMF exposure, the particles were removed by magnetic separation and an aliquot of the final supernatant was collected for analysis.

The collected aliquots, both pre- and post-AMF exposure, were identically diluted and their fluorescence was measured during reverse phase HPLC using a Waters Atlantis C18 column ( $5 \mu \mathrm{m}, 3.9 \times 150 \mathrm{~mm})$ on an Agilent 1100 series HPLC. The total run-time was 45 minutes with the following gradient: (\% line $B$, elapsed time in minutes) 0,$0 ; 0,3 ; 60,10$; 80,$25 ; 100,30 ; 100,35 ; 0,45$ where the $A$ line was $5 \mathrm{mM}$ ammonium acetate $(\mathrm{pH} 6)$ and the 
B line was $75 \%$ v/v aqueous acetonitrile. Excitation-emission spectra (EES) of FITC-CHO, FITC-I, and FITC-II were acquired on a PerkinElmer LS55 fluorescence spectrometer in order to determine the excitation wavelengths that would yield maximum emission for each adduct. The fluorescence excitation beam-width of the Agilent instrument was $20 \mathrm{~nm}$.

5.3.10 Syntheses of doxorubicin analogs

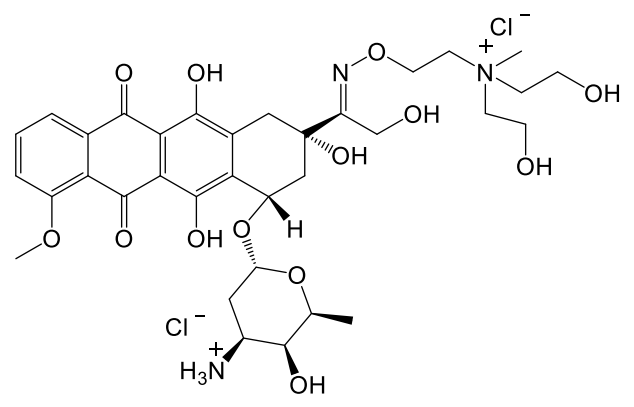

Synthesis of Dox-2OH

To a solution of doxorubicin $\bullet \mathrm{HCl}(105.3 \mathrm{mg}, 0.18 \mathrm{mmol})$ in anhydrous methanol (9 $\mathrm{mL})$ at room temperature was added $\mathrm{QAO}-2 \mathrm{OH}(162.3 \mathrm{mg}, 0.54 \mathrm{mmol})$ and trifluoroacetic $\operatorname{acid}(9.0 \mu \mathrm{L})$. After stirring the reaction mixture in the dark at room temperature for $110 \mathrm{~h}$, the solvent was removed by rotary evaporation. The product was purified from the crude mixture by $\mathrm{C} 18$ reverse phase flash chromatography (C18Aq) with gradient elution in water/methanol with $0.1 \%$ TFA. The product eluted at $55 \%$ water $45 \%$ methanol/TFA. A hygroscopic red solid was isolated. The residue was dissolved in methanol and gravity eluted through an ion exchange resin as previously described to yield a red solid $(0.13 \mathrm{~g}$, 93\%). ${ }^{1} \mathrm{H}$ NMR (500 MHz, CD $\left.30 \mathrm{OD}\right) \delta 1.28(\mathrm{~d}, \mathrm{~J}=5,3 \mathrm{H}) ; 1.91(\mathrm{~m}, 1 \mathrm{H}) ; 2.01(\mathrm{~m}, 1 \mathrm{H}) ; 2.18(\mathrm{~d}$, $J=5.0 \mathrm{~Hz}, 1 \mathrm{H}) ; 2.45(\mathrm{~d}, J=15 \mathrm{~Hz}, 1 \mathrm{H}) ; 2.90,3.01(\mathrm{ABq}, 2 \mathrm{H}, J \mathrm{AB}=17.5 \mathrm{~Hz}) ; 3.27(\mathrm{~m}, 1 \mathrm{H})$; 
3.30 (s 3H); 3.60 (d, J= 15 Hz, $1 \mathrm{H}) ; 3.67-3.75$ (m, 6H); 3.82 (bs, 3H); 3.91-4.05 (m, 9H); 4.23 $(\mathrm{m}, 1 \mathrm{H}) ; 4.49,4.53(\mathrm{ABq}, 2 \mathrm{H}, J \mathrm{AB}=15 \mathrm{~Hz}) ; 4.63(\mathrm{~m}, 4 \mathrm{H}) ; 4.74$ (splitting and integration obscured by water peak, presumed to be $1 \mathrm{H}$ on $\mathrm{C} 7$, according to ref); ${ }^{170} 5.35$ (bs, $\left.1 \mathrm{H}\right), 7.23$ $(\mathrm{d}, J=10 \mathrm{~Hz}, 1 \mathrm{H}) ; 7.43(\mathrm{~d}, J=10 \mathrm{~Hz}, 1 \mathrm{H}) ; 7.51(\mathrm{t}, J=7.5 \mathrm{~Hz}, 1 \mathrm{H}) .{ }^{13} \mathrm{C}$ NMR $\left(125 \mathrm{MHz}, \mathrm{CD}_{3} \mathrm{OD}\right)$ $\delta 185.9,185.63,162.9,160.7,156.0,154.5,135.7,134.8,134.7,134.3,119.5,118.8$ $118.7,110.5,110.3,99.5,71.6,70.8,67.7,66.6,66.4,64.9,62.2,55.8,55.4,54.2,49.9$, 37.7, 33.2, 28.1, 15.9. $\mathrm{m} / \mathrm{z}$ calcd $\left[\mathrm{C}_{34} \mathrm{H}_{46} \mathrm{~N}_{3} \mathrm{O}_{13}\right]^{+}$704.302518, observed 704.30269.

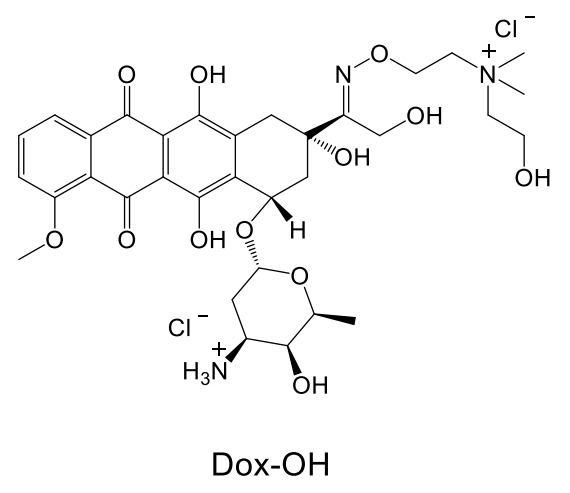

Synthesis of Dox-OH

To a solution of doxorubicin $\bullet \mathrm{HCl}(103.0 \mathrm{mg}, 0.178 \mathrm{mmol})$ in anhydrous methanol $(9 \mathrm{~mL})$ at room temperature was added $\mathrm{QAO}-1 \mathrm{OH}(146 \mathrm{mg}, 0.533 \mathrm{mmol})$ and trifluoroacetic acid $(9.0 \mu \mathrm{L})$. After stirring the reaction mixture in the dark at room temperature for $72 \mathrm{~h}$, the solvent was removed by rotary evaporation. The product was purified from the crude mixture by C18 reverse phase flash chromatography (C18Aq) with gradient elution in water/methanol with $0.1 \%$ TFA. The product eluted at $55 \%$ water $45 \%$ methanol/TFA. The hygroscopic red solid was then converted to a chloride salt by gravity eluting through an ion exchange resin prepared as described previously ( 0.1348 g, quant). ${ }^{1} \mathrm{H}$ NMR (500 MHz, CD $\left.30 \mathrm{OD}\right) \delta 1.28(\mathrm{~d}, J=6,3 \mathrm{H}) ; 1.91(\mathrm{~m}, 1 \mathrm{H}) ; 2.01(\mathrm{~m}, 1 \mathrm{H}) ; 2.23(\mathrm{~m}, 1 \mathrm{H})$; 
$2.50(\mathrm{br} \mathrm{d}, J=14 \mathrm{~Hz}, 1 \mathrm{H}) ; 3.03(\mathrm{~m}, 2 \mathrm{H}) ; 3.26(\mathrm{~d}, J=3.5 \mathrm{~Hz}, 6 \mathrm{H}) ; 3.58(\mathrm{~m} \mathrm{3H}) ; 3.72(\mathrm{br} \mathrm{s}, 1 \mathrm{H})$; $3.88(\mathrm{~m}, 5 \mathrm{H}) ; 3.99(\mathrm{~m}, 2 \mathrm{H}) ; 4.25(\mathrm{~m}, 1 \mathrm{H}) ; 4.49,4.55(\mathrm{ABq}, 2 \mathrm{H}, \mathrm{JAB}=13.3 \mathrm{~Hz}) ; 4.61(\mathrm{~m}, 2 \mathrm{H})$; $5.37(\mathrm{~m}, 1 \mathrm{H}), 7.33(\mathrm{~m}, 1 \mathrm{H}) ; 7.59(\mathrm{~m}, 2 \mathrm{H})$. One ${ }^{1} \mathrm{H}$ signal was obscured by water contamination in methanol, but was verified in DMF- $d_{7}$ at $\delta 5.15 .{ }^{13} \mathrm{C} \mathrm{NMR}(125 \mathrm{MHz}$, $\left.\mathrm{CD}_{3} \mathrm{OD}\right) \delta 15.8,22.6,23.5,28.1,28.7,30.2,33.2,37.9,38.7,51.9,54.3,55.5,55.8,63.8$ $66.4,66.6,67.7,67.8,71.0,71.7,99.6,110.5,110.7,118.9,118.9,119.8,135.0,135.8$ 154.7, 156.1, 160.9, 163.0, 186.0, 186.3. $\mathrm{m} / \mathrm{z}$ calcd $\left[\mathrm{C}_{33} \mathrm{H}_{44} \mathrm{~N}_{3} \mathrm{O}_{12}\right]^{+} 674.291953$, observed 674.29305.

\subsubsection{Preparation of NP formulations of Dox analogs}

To NPs (30.1 and $29.9 \mathrm{mg}$ ) was added $1.5 \mathrm{~mL}$ of either Dox-OH or Dox-2OH (respectively) as $0.1 \mathrm{M}$ solutions in anhydrous DMSO such that $5 \mu$ mols Dox analog per 1 mg NP was added. Vials were vortex mixed, then inverted (Labquake microcentrifuge tube shaker, Barnstead Thermolyne) at room temperature for $12 \mathrm{~h}$. The drug-loaded particles were then magnetically separated from the supernatant and washed with methanol (2X $1.5 \mathrm{~mL})$ and water $(2 \mathrm{X} 1.5 \mathrm{~mL})$ to afford NP.Dox-OH $(30.9 \mathrm{mg})$ and NP.Dox$2 \mathrm{OH}$ (30.3 mg). 


\subsubsection{UV-Vis detection of Dox-OH and Dox-2OH release by pulsed AMF}

$2.5 \mathrm{mg} / \mathrm{mL}$ solutions of either NP.Dox-OH or NP.Dox-2OH were prepared in $1 \mathrm{X}$ phosphate buffered saline and added to polystyrene cuvettes (Brookhaven Instruments). The cuvettes were placed, one per trial, in the center of an induction coil (Easy Heat LI AMF generator). The solutions were exposed to AMF pulses lasting five minutes $(203 \mathrm{kHz}$, 350 A) at room temperature. After each pulse, the NPs were magnetically sedimented and the cuvettes were placed in a $30^{\circ} \mathrm{C}$ spectrophotometer (Beckman Coulter DU 800) where absorbance at $480 \mathrm{~nm}$ was detected. Absorbance at $380 \mathrm{~nm}$ was used for baseline correction. A total of 6 five-minute pulses were applied with intervening 5 minute periods of "off" time for a total time of $1 \mathrm{~h}$ per experiment. Trials were performed in triplicate.

\subsubsection{Toxicity of Dox-NPs in MCF-7 cells}

MCF-7 breast cancer cells were cultured in DMEM, 10\% FBS, and 1\% Pen strep and were plated into one of two 96 well plates which had been cut down to fit within the coil of the AMF generator. NP.Dox-OH, NP.Dox-2OH, or bare NPs (control) were dispersed in PBS buffer ( $50 \mu \mathrm{L} /$ well) and then diluted with supplemented medium (200 $\mu \mathrm{L} /$ well). When cells had grown to $40 \%$ confluence, the medium was removed and replaced with the NP formulations. One 96 well plate was placed within the coil of the AMF generator and cells were exposed to 15 minutes pulsed AMF (350A, $203 \mathrm{kHz}, 3 \mathrm{~min}$ on, $3 \mathrm{~min}$ off, 30 min total time). Between pulses cells were incubated at $37^{\circ} \mathrm{C}$. After 48 hours, toxicity was assayed by trypan blue exclusion. The medium was removed, wells were washed $2 \mathrm{X}$ with $200 \mu \mathrm{L}$ PBS. $100 \mu \mathrm{L}$ trypsin was added and the plates were incubated for $5 \mathrm{~min} .100$ 
$\mu \mathrm{L}$ medium was added to neutralize the trypsin and the contents of the wells were transferred to microcentrifuge tubes. The cells were pelleted by centrifuge (1100 rpm, 5 $\min$ ), and the supernatant was aspirated. Cell pellets were dispersed in $30 \mu \mathrm{L}$ PBS buffer plus $10 \mu \mathrm{L}$ trypan blue. Live cells, which had excluded the blue dye, were counted using a hemocytometer.

5.3.14 Synthesis of QAO-2OH anthracene derivative<smiles></smiles>

To aminooxy precursor QAO-2OH $(0.405 \mathrm{~g}, 1.89 \mathrm{mmol})$ in 2:1 $\mathrm{MeOH}: \mathrm{CH}_{2} \mathrm{Cl}_{2}(25$ $\mathrm{mL}$ ) was added 9-anthracenecarboxaldehyde $(0.582 \mathrm{~g}, 2.82 \mathrm{mmol})$. The mixture was stirred at $\mathrm{rt}$ for $48 \mathrm{~h}$. The solvent was removed by rotary evaporation. The residue was passed through a thin silica gel plug eluting with $\mathrm{CH}_{2} \mathrm{Cl}_{2}: \mathrm{MeOH}(9: 1)$. The solvent was evaporated by rotary evaporation to yield the product as a viscous yellow liquid $(0.263 \mathrm{~g}$, 35\%). ${ }^{1} \mathrm{H}$ NMR $E$ isomer $\left(400 \mathrm{MHz}, \mathrm{CD}_{3} \mathrm{OD}\right) \delta 9.08(\mathrm{~s}, 1 \mathrm{H}), 8.31(\mathrm{~d}, J=9.6 \mathrm{~Hz}, 2 \mathrm{H}), 7.87$ (d, $J=8.4 \mathrm{~Hz}, 1 \mathrm{H}), 7.55(\mathrm{t}, J=7.6 \mathrm{~Hz}, 2 \mathrm{H}), 7.53(\mathrm{t}, J=7.4 \mathrm{~Hz}, 2 \mathrm{H}), 4.72(\mathrm{br} \mathrm{s}, 2 \mathrm{H}), 4.04(\mathrm{~m}, 4 \mathrm{H})$, $3.96(\mathrm{~m}, 2 \mathrm{H}), 3.70(\mathrm{~m}, 4 \mathrm{H}), 3.31(\mathrm{~s}, 3 \mathrm{H}) .{ }^{13} \mathrm{C} N M R E$ isomer $\left(100 \mathrm{MHz}, \mathrm{CD}_{3} \mathrm{OD}\right) \delta$ 150.8; $132.5 ; 131.2 ; 130.9 ; 130.0 ; 128.1 ; 126.4 ; 125.8 ; 123.7 ; 68.8 ; 66.2 ; 63.6 ; 56.8 ; 51.2$. 


\subsubsection{Dextran sulfate coating of NPs}

$49.5 \mathrm{mg}$ NPs were dispersed in $100 \mathrm{~mL}$ DMSO. $100.3 \mathrm{mg}$ dextran sulfate in DMSO $(100 \mathrm{~mL})$ was added to the NPs with rapid stirring slowly by addition funnel. The mixture was stirred $14 \mathrm{~h}$ at rt. NPs were magnetically separated, the supernatant was removed by aspiration, the precipitated particles were washed $2 \mathrm{X}$ with Millipore water and freeze dried.

\subsubsection{Coating of dextran sulfate NPs with QAO-2OH anthracene derivative}

To $19.9 \mathrm{mg}$ dextran sulfate-coated NPs in DMSO (30 mL) was added QAO-2OH anthracene derivative $(0.040 \mathrm{~g}, 0.10 \mathrm{mmol})$. The mixture was stirred at $\mathrm{rt}$ for $12 \mathrm{~h}$. Coated NPs were magnetically separated, the supernatant was removed by aspiration, the precipitated particles were washed $2 \mathrm{X}$ with Millipore water and freeze dried.

\subsubsection{Release of QAO-2OH anthracene derivative from dextran sulfate NPs}

A $2.5 \mathrm{mg} / \mathrm{mL}$ aqueous solution of the $\mathrm{QAO}-2 \mathrm{OH}$ anthracene derivative-loaded dextran sulfate NPs was placed in a cuvette. The solution was vortex mixed and the particles were magnetically sedimented. Absorbance of the solution phase was measured at $382 \mathrm{~nm}$ UV-Vis. This procedure was repeated every 5 minutes for $30 \mathrm{~min}$ at $\mathrm{rt}$. The solution was then exposed to pulsed AMF for 30 minutes ( 5 min on, 2.5 min off). At 5 min intervals after each pulse the particles were again magnetically separated and absorbance at $382 \mathrm{~nm}$ was measured. 


\subsubsection{8 $\mathrm{CS}_{2}$ and $\mathrm{CS}_{2} / \mathrm{CH}_{3}$ l coating of NPs}

In a typical procedure for coating NPs with $\mathrm{CS}_{2}, 20-60 \mathrm{mg}$ NPs would be heated in a Kügelrohr distillation apparatus under vacuum at $125^{\circ} \mathrm{C}$ for 4 hours to remove surfacebound water. A pre-heated stir bar and $\mathrm{CS}_{2}(2-3 \mathrm{~mL})$ would then be added and the solution would be stirred for $4 \mathrm{~h}$ at $\mathrm{rt}$. The particles would then be magnetically separated and dried under vacuum. For methyl capping, iodomethane (1-2 $\mathrm{mL})$ would be added to the particles and the solution would be stirred for $12 \mathrm{~h}$ at $\mathrm{rt}$. Excess iodomethane would be evaporated under $\mathrm{N}_{2}$ stream in a fume hood and, following that, the coated NPs would be dried under vacuum for up to $48 \mathrm{~h}$.

\subsubsection{Synthesis of deuterated ammonium salt 3}

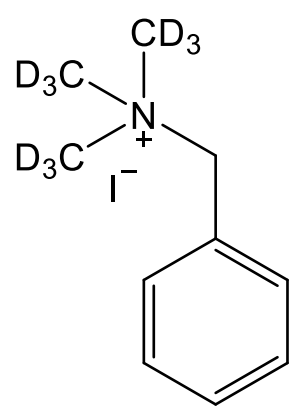

Following a modified procedure by Sommer et al., ${ }^{171}$ to benzylamine $(0.49 \mathrm{~g}, 4.57$ $\mathrm{mmol})$ in DMF $(2.3 \mathrm{~mL})$ was added $N, N$-diisopropylethylamine $(1.6 \mathrm{~mL}, 9.15 \mathrm{mmol})$. The mixture was stirred for 15 minutes and cooled to $0{ }^{\circ} \mathrm{C}$ with an ice bath. $\mathrm{CD}_{3} \mathrm{l}(2.99 \mathrm{~g}, 20.6$ $\mathrm{mmol}$ ) was added dropwise and the mixture was allowed to come to rt and stirred $12 \mathrm{~h}$. After removal of excess $\mathrm{CD}_{3} \mathrm{l}$ in vaccuo, hot EtOAc was added and the reaction was filtered of a white precipitate. The filtrate was concentrated by rotary evaporation and the DMF removed by azeotrope with toluene. The product was isolated by reverse phase flash 
column chromatography (RediSep Rf Gold C18Aq) as a white solid (1.07 g, 82\%). ${ }^{1} \mathrm{H}$ NMR $\left(400 \mathrm{MHz}, \mathrm{CD}_{3} \mathrm{OD}\right) \delta 7.79(\mathrm{~d}, J=8.4 \mathrm{~Hz}, 2 \mathrm{H}) ; 7.69-7.60(\mathrm{~m}, 3 \mathrm{H}) ; 4.90(\mathrm{~s}, 2 \mathrm{H}) ;{ }^{13} \mathrm{C}$ NMR $(400$ $\left.\mathrm{MHz}, \mathrm{CD}_{3} \mathrm{OD}\right) \delta 133.8 ; 131.7 ; 130.0 ; 128.6 ; 69.2,52.2(\mathrm{~m}) ; \mathrm{m} / \mathrm{z}$ calcd $\left[\mathrm{C}_{33} \mathrm{H}_{44} \mathrm{~N}_{3} \mathrm{O}_{12}\right]^{+}$ 674.291953, observed 674.29305.

\subsubsection{Coating of $\mathrm{CS}_{2}$ NPs with 3}

$20.8 \mathrm{mg}$ NPs were heated at $125^{\circ} \mathrm{C}$ under vacuum in a Kügelrohr. $0.7 \mathrm{~mL} \mathrm{CS} 2$ was added and the solution was stirred at $\mathrm{rt}$ for several hours. Excess $\mathrm{CS}_{2}$ was evaporated under $\mathrm{N}_{2}$ stream. $0.63 \mathrm{~mL}$ of a $0.1 \mathrm{M}$ solution in DMSO of the ammonium salt was added and the solution was stirred $19 \mathrm{~h}$. The particles were then magnetically separated and the supernatant removed. Particles were washed with $2 \times 1 \mathrm{~mL}$ water and freeze dried. As a control, NPs were treated as above without the addition of $\mathrm{CS}_{2}$. TGA analysis was performed on $\approx 5 \mathrm{mg}$ of sample for three conditions: $\mathrm{NPs}+\mathrm{CS}_{2}, \mathrm{NPs}+\mathrm{CS}_{2}+3$, or NPs+ 3 . 
5.3.21 Synthesis of anthracen-9-ylmethanamine (4)<smiles>NCc1c2ccccc2cc2ccccc12</smiles>

Reagents and conditions: (a) NaBH4 (4.8 eq), THF/MeOH, rt

2.5 h; (b) Phthalimide, $\mathrm{PPh}_{3}, \mathrm{DIAD}, \mathrm{THF}, 0{ }^{\circ} \mathrm{C}-\mathrm{rt}, 16 \mathrm{~h}$; (c)

$\mathrm{H}_{2} \mathrm{NNH}_{2}$ monohyd., $\mathrm{CH}_{2} \mathrm{Cl}_{2}: \mathrm{MeOH}, \mathrm{rt}, 14 \mathrm{~h}$<smiles>OCc1c2ccccc2cc2ccccc12</smiles>

To 9-anthracene carboxaldehyde $(2.51 \mathrm{~g}, 12.2 \mathrm{mmol})$ in 5:1 THF:MeOH $(60 \mathrm{ml})$ was slowly added $\mathrm{NaBH}_{4}$. The mixture was stirred $2.5 \mathrm{~h}$ after which the reaction was quenched with aq. $\mathrm{NH}_{4} \mathrm{Cl}$. The precipitate was removed by filtration and the filtrate extracted with diethyl ether $(3 \mathrm{X}, 50 \mathrm{~mL})$. The combined organic phases were washed with brine and dried over sodium sulfate. The product was purified by column chromatography (EtOAc/CH2Cl3) to yield a yellow solid. Rf 0.55 (1:20 EtOAc: $\mathrm{CH}_{2} \mathrm{Cl}_{2}$ ). ${ }^{1} \mathrm{H} \mathrm{NMR}(400 \mathrm{MHz}$, $\left.\mathrm{CDCl}_{3}\right) \delta=8.47(\mathrm{~m}, 3 \mathrm{H}) ; 8.03(\mathrm{~d}, J=8.4 \mathrm{~Hz}, 2 \mathrm{H}) ; 7.57(\mathrm{~m}, 2 \mathrm{H}) ; 7.49(\mathrm{~m}, 2 \mathrm{H}) 5.68(\mathrm{~d}, J=5.2$ $\mathrm{Hz}, 2 \mathrm{H}), 1.73(\mathrm{br}, 1 \mathrm{H})$. 
<smiles>NCc1c2ccccc2cc2ccccc12</smiles>

To a solution of triphenylphosphine $(2.82 \mathrm{~g}, 10.77 \mathrm{mmol})$, phthalimide $(1.58 \mathrm{~g}$, $10.77 \mathrm{mmol})$, and anthracene methanol in anhydrous THF $(40 \mathrm{~mL})$ at $0{ }^{\circ} \mathrm{C}$ was added dropwise DIAD (2.18 g, $10.77 \mathrm{mmol})$. The mixture was stirred at $\mathrm{rt}$ for $16 \mathrm{~h}$. THF was removed by rotary evaporation. The yellow solid was triturated in $50 \mathrm{~mL}$ rt diethyl ether and the precipitated solids were collected over a Buchner funnel giving a yellow solid that was used directly in the next step. ${ }^{1} \mathrm{H}$ NMR $(400 \mathrm{MHz}, \mathrm{CDCl} 3) \delta 5.87(\mathrm{~s}, 2 \mathrm{H}), 7.48(\mathrm{t}, J=$ 7.4 Hz, 2H), 7.57-7.66 (m, 4H), 7.75-7.77 (m, $2 \mathrm{H}) ; 8.01(\mathrm{~d}, J=8.8 \mathrm{~Hz}, 2 \mathrm{H}), 8.48(\mathrm{~s}, 1 \mathrm{H}) ; 8.66$ $(\mathrm{d}, J=9.2 \mathrm{~Hz}, 2 \mathrm{H})$.

The anthryl phthalimide $\left(0.667 \mathrm{~g}, 1.98 \mathrm{mmol}\right.$ ) was dissolved in $2: 1 \mathrm{CH}_{2} \mathrm{Cl}_{2}: \mathrm{EtOH}$ (50 $\mathrm{mL})$. Hydrazine monohydrate $(0.495 \mathrm{~g}, 9.89 \mathrm{mmol})$ was added and the mixture was stirred for $14 \mathrm{~h}$ at rt. The product was purified by silica gel chromatography $(9: 1: 0.1 \mathrm{CH} 2 \mathrm{Cl} 2$ : MeOH: $\mathrm{NH} 4 \mathrm{OH}$ ) to yield an amber solid (16\%, 2 step). ${ }^{1} \mathrm{H} \mathrm{NMR}(400 \mathrm{MHz}, \mathrm{CDCl} 3) \delta 1.70$ (bs, $2 \mathrm{H}) ; 4.82(\mathrm{~s}, 2 \mathrm{H}) ; 7.46-7.57(\mathrm{~m}, 4 \mathrm{H}) ; 8.02(\mathrm{~d}, \mathrm{~J}=8.4 \mathrm{~Hz}, 2 \mathrm{H}) ; 8.33-8.40(\mathrm{~m}, 3 \mathrm{H})$. 


\subsubsection{Preparation of thiocarbamate NPs 10}

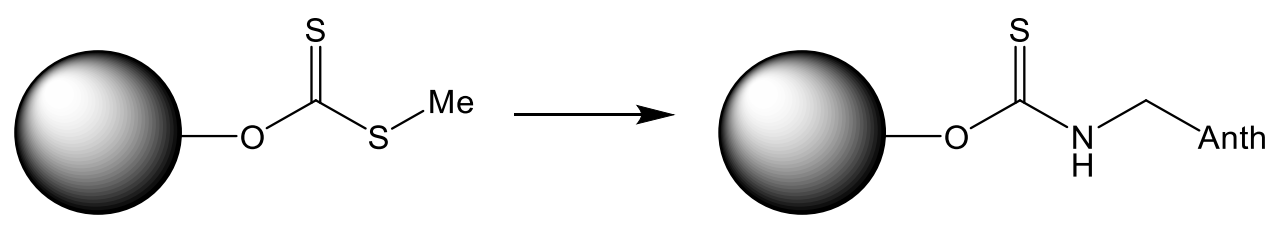

To xanthate ester NPs $8(35.6 \mathrm{mg})$ in THF $(2 \mathrm{~mL})$ was added $20 \mathrm{mg}$ of 4 in $0.3 \mathrm{~mL}$. The mixture was magnetically stirred at $\mathrm{rt}$ for $6 \mathrm{~h}$. The coated particles 10 (which had changed color from brown to beige) were magnetically sedimented. The supernatant was removed and the particles were washed with THF. With successive washing the particles lost their pale coloring.

\subsubsection{Elemental analysis}

Elemental analyses were performed on $\approx 50 \mathrm{mg}$ of sample by Midwest Microlab, LLC.

5.3.24 $\mathrm{CS}_{2}$-modified NPs with variably hydroxylated ammonium salts

A stock solution of $\mathrm{CS}_{2}$ modified NPs in DMSO was aliquoted into 15 microcentrifuge tubes such that each tube contained $\approx 10 \mathrm{mg}$ particles. $0.15 \mathrm{~mL}$ of $0.66 \mathrm{M}$ solutions of each ammonium salt (Figure 3.3) were added to the particle solutions and the mixtures were vortex mixed, sonicated ( $5 \mathrm{~min}$ ) and placed on inverter for mixing for 20h. The coated NPs were then magnetically separated, the supernatant was removed and the particles were washed with water $(3 \times 0.5 \mathrm{~mL})$ with intervening pelleting by centrifugation (13000 rpm, rt, $5 \mathrm{~min}$ ) and supernatant removal. The washed particles were freeze dried. An unidentified orange solid was seen to pellet with the NPs. 


\subsubsection{Synthesis of greigite NPs}

Following a procedure describe by Chang et al., ${ }^{123} 6.5 \mathrm{~mL}$ water was deoxygenated with $\mathrm{N}_{2}$ bubbling for $45 \mathrm{~min}$. Iron II sulfate $(1.82 \mathrm{~g}, 6.54 \mathrm{mmol})$ and $\mathrm{Na}_{2} \mathrm{~S} \cdot 9 \mathrm{H}_{2} \mathrm{O}(1.57 \mathrm{~g}$, $6.54 \mathrm{mmol}$ ) were added and a black precipitate formed immediately. Glacial acetic acid was then added dropwise as the solution $\mathrm{pH}$ was monitored, until the $\mathrm{pH}$ reached 3.3. Greigite NPs were pelleted by centrifugation (5000 rpm, $5 \mathrm{~min}, \mathrm{rt}$ ). After supernatant removal the particles were washed with deoxygenated water $(40 \mathrm{~mL})$ and again pelleted by centrifuge. The supernatant was once again removed and the particles were freeze dried. The particles were not immediately responsive to a magnet, however after sitting overnight at rt in a vial, they became magnetic.

\subsubsection{Greigite NP coating with QAO-2OH anthracene derivative}

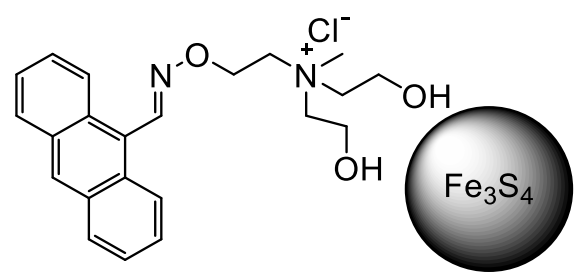

To $1 \mathrm{~mL}$ of QAO-2OH anthracene derivative $(25 \mathrm{mg} / \mathrm{mL})$ in andhyd. DMSO was added $50.6 \mathrm{mg}$ greigite NPs. The mixture was stirred at $\mathrm{rt}$ for $48 \mathrm{~h}$. Coated particles were pelleted by centrifugation (13000 rpm, $15 \mathrm{~min}, \mathrm{rt}$ ). The supernatant was removed and the particles were washed with water $(3 X 1 \mathrm{~mL})$ with intervening centrifugation and supernatant removal. The particles were then freeze dried. 


\subsection{Experimental Procedures for Chapter 4}

5.4.1 Syntheses of cationic oxime ether lipids and intermediates

5.4.2 Synthesis of doxorubicin hexadecanyl acyl hydrazone (Dox-AH)

5.4.3 Synthesis of core-shell $\mathrm{Fe}_{3} \mathrm{O}_{4}-\mathrm{SiO}_{2} \mathrm{NPs}$

5.4.4 CML formation and stability

5.4.5 ऊ-potential of CML

5.4.6 Calculation of lipid molecules per NP

5.4.7 Titration of SNP with cationic lipid 1

5.4.8 Estimation of lipid: Dox-AH ratio

5.4.9 Cell toxicity study

5.4.10 Preparation of oxime-ether liposomes and nucleic acid complexes

5.4.11 Cell culture for gene transfection studies

5.4.12 Binding affinity of oxime ether lipids with hybrid DNA/RNA

5.4.13 Cell viability for gene transfection studies

5.4.14 Transfection and GFP silencing assay 


\subsection{Experimental Procedures for Chapter 4}

5.4.1 Syntheses of cationic oxime ether lipids and intermediates<smiles>NOCCN(CCON)C(=O)OCc1ccccc1</smiles>

tert-butyl $N, N$-bis[2-(aminooxy)ethyl]carbamate (4.1).

[Steps $a, b$ ] To a solution of tert-butyl bis(2-hydroxyethyl)carbamate $(5.01 \mathrm{~g}, 24.4$ mmol), triphenylphosphine $(14.1 \mathrm{~g}, 53.7 \mathrm{mmol})$ and $N$-hydroxyphthalimide $(8.75 \mathrm{~g}, 53.6$ $\mathrm{mmol})$ in THF $(245 \mathrm{~mL})$ at $0{ }^{\circ} \mathrm{C}$ was added dropwise diisopropyl azodicarboxylate (DIAD) (10.6 $\mathrm{g}, 52.4 \mathrm{mmol})$. The resultant red mixture was allowed to warm to rt. After stirring for $12 \mathrm{~h}$, the solvent was removed by rotary evaporation. $\mathrm{Et}_{2} \mathrm{O}(150 \mathrm{~mL})$ was added and the mixture was cooled to $0{ }^{\circ} \mathrm{C}$ for $3 \mathrm{~h}$. The resultant suspension was decanted to remove an off-white precipitate. The decanted solution was chilled to $-20^{\circ} \mathrm{C}$ for another $3 \mathrm{~h}$ and again decanted to remove a second crop of precipitated solids. The solvent then was removed by rotary evaporation and a mixture of hexanes:EtOAc, 1:1 (60 mL) was added to the crude residue. The precipitated solids were collected using a Buchner funnel, dissolved in a minimal volume of $\mathrm{CH}_{2} \mathrm{Cl}_{2}$, and passed through a plug of silica gel, eluting with a gradient of $\mathrm{CH}_{2} \mathrm{Cl}_{2}$ : EtOAc, 1:0 to $0: 1$. The isolated product containing minor impurities was recrystallized by first dissolving in a minimal volume of hot EtOAc and then adding hexanes (final ratio: 2:1 hexanes/EtOAc). The bis-phthalimide, tert-butyl $\mathrm{N}, \mathrm{N}$ $\operatorname{bis}(\{2-[1,3-d i o x o-2,3-d i h y d r o-1 H$-isoindol-2-yl)oxy]ethyl\})carbamate, obtained as a white solid $(2.07 \mathrm{~g}, 17 \%)$ was used directly in the next step; m.p. $139-141{ }^{\circ} \mathrm{C} ; \mathrm{TLC}, \mathrm{R}_{\mathrm{f}} 0.47$ 
(hexanes/EtOAc, 1:1, UV/ninhydrin stain); FT-IR 2972, 2956, 1793, 1736, 1729, 1697, 1412

$\mathrm{cm}^{-1} ;{ }^{1} \mathrm{H}$ NMR (400 MHz, $\left.\mathrm{CDCl}_{3}\right) \delta 1.41(\mathrm{~s}, 9 \mathrm{H}) ; 3.73(\mathrm{~m}, 4 \mathrm{H}) ; 4.36(\mathrm{~m}, 4 \mathrm{H}) ; 7.71(\mathrm{~m}, 8 \mathrm{H}) ;$

${ }^{13} \mathrm{C}$ NMR $\left(125 \mathrm{MHz}, \mathrm{CDCl}_{3}\right) \delta 28.5 ; 47.7 ; 78.0,80.5 ; 123.6 ; 129.1 ; 134.6 ; 155.4 ; 163.5$.

To a solution of the isolated bis phthalimide carbamate $(3.10 \mathrm{~g}, 6.26 \mathrm{mmol})$ in $\mathrm{CH}_{2} \mathrm{Cl}_{2}(33 \mathrm{~mL})$ at $\mathrm{rt}$ was added dropwise hydrazine monohydrate $(1.57 \mathrm{~g}, 31.3 \mathrm{mmol})$. After stirring at $\mathrm{rt}$ for $2.5 \mathrm{~h}$, the reaction mixture was filtered to remove the white precipitate, and the retentate was washed with small portions of $\mathrm{CH}_{2} \mathrm{Cl}_{2}$. The combined filtrate was evaporated by rotary evaporation to afford bis-aminooxy carbamate 4.1 as a clear oil (1.42 g, 96\%); TLC, $\mathrm{R}_{\mathrm{f}} 0.74\left(\mathrm{CH}_{2} \mathrm{Cl}_{2}: \mathrm{MeOH}, 9: 1\right) ; \mathrm{FT}-\mathrm{IR} 3312,2976,2925,2871$,

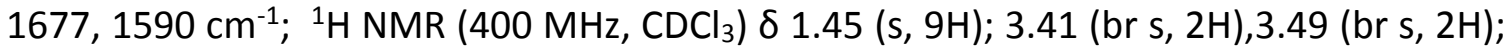
$3.74(\mathrm{t}, J=5.2 \mathrm{~Hz}, 4 \mathrm{H}) ; 5.46\left(\mathrm{br} \mathrm{s}, 4 \mathrm{H},-\mathrm{ONH}_{2}\right) ;{ }^{13} \mathrm{C} \mathrm{NMR}\left(100 \mathrm{MHz}, \mathrm{CDCl}_{3}\right) \delta$ 28.4; 46.0; 46.2; 73.2; 74.0; 79.7; 155.8. HRMS $\mathrm{m} / \mathrm{z}$ calcd $\left[\mathrm{C}_{9} \mathrm{H}_{22} \mathrm{~N}_{3} \mathrm{O}_{4}\right]^{+}[\mathrm{M}+\mathrm{H}]^{+}:$236.16048, observed 236.16065. 


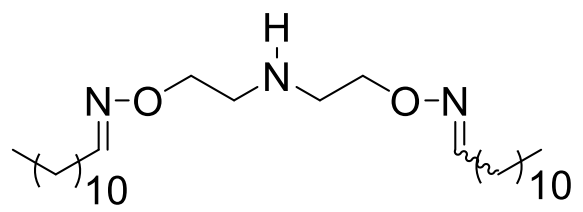

(21E)-14,20-dioxa-13,17,21-triazatritriaconta-12,21-diene (4.2)

Bis-aminooxy carbamate $4.1(0.10 \mathrm{~g}, 4.21 \mathrm{mmol})$ was dissolved in $\mathrm{CH}_{2} \mathrm{Cl}_{2}(20 \mathrm{~mL})$. To this solution was added dodecyl aldehdye $(1.71 \mathrm{~g}, 9.26 \mathrm{mmol})$, prepared from dodecyl alcohol by Swern oxidation. ${ }^{172}$ After stirring the reaction for $10 \mathrm{~h}$ at $\mathrm{rt}$, the solvent was removed by rotary evaporation and the crude product was purified by silica column chromatography $\left(\mathrm{CH}_{2} \mathrm{Cl}_{2}\right.$ : EtOAc, 1:0 to 9:1) to afford 4.2 (2.23 g, 93\%) as a colorless oil. The mixture of $(E, E):(E, Z)$ diastereomers was used directly in the next step without isomer separation; TLC, $\mathrm{R}_{\mathrm{f}}$ 0.53, $0.42\left(\mathrm{CH}_{2} \mathrm{Cl}_{2} /\right.$ EtOAc, 9:1, $p$-anisaldehyde stain); ${ }^{1} \mathrm{H}$ NMR (400 $\left.\mathrm{MHz}, \mathrm{CDCl}_{3}\right)(E, E)$-isomer (major): $\delta 0.88(\mathrm{t}, J=5.6 \mathrm{~Hz}, 6 \mathrm{H}) ; 1.25(\mathrm{~m}, 34 \mathrm{H}), 1.45(\mathrm{~s}, 9 \mathrm{H}) ; 1.62$ $(\mathrm{m}, 2 \mathrm{H}) ; 2.17(\mathrm{q}, J=6.2 \mathrm{~Hz}, 4 \mathrm{H}) ; 3.47(\mathrm{~m}, 4 \mathrm{H}) ; 4.11(\mathrm{~m}, 4 \mathrm{H}) ; 7.37(\mathrm{t}, J=6.0 \mathrm{~Hz}, 2 \mathrm{H}) ;{ }^{13} \mathrm{C} \mathrm{NMR}$ $\left(100 \mathrm{MHz}, \mathrm{CDCl}_{3}\right) \delta 14.1,22.6,25.7,26.2,26.7,28.4,29.1,29.3,29.4,29.5,29.6,29.8,31.9$ $47.3,71.7,72.2,79.5,151.3,152.3,155.4$.

The bis-oxime ether $4.2(1.57 \mathrm{~g}, 2.77 \mathrm{mmol})$ was dissolved in a chilled 1:1 mixture of trifluoro-acetic acid: $\mathrm{CH}_{2} \mathrm{Cl}_{2}(7.0 \mathrm{~mL})$ and the mixture was stirred at $\mathrm{rt}$ for 3 hours. The solvent was evaporated by rotary evaporation and $\mathrm{Et}_{2} \mathrm{O}(50 \mathrm{~mL})$ was added to dissolve the residue. The $\mathrm{Et}_{2} \mathrm{O}$ solution was washed with sat. $\mathrm{NaHCO}_{3}(50 \mathrm{~mL})$. The aqueous wash layer was extracted with $\mathrm{Et}_{2} \mathrm{O}(2 \times 30 \mathrm{~mL})$ and the combined $\mathrm{Et}_{2} \mathrm{O}$ phase was dried $\left(\mathrm{NaSO}_{4}\right)$ and then evaporated by rotary evaporation to yield bis-oxime ether amine (1.25 g, 96\%) as an amber liquid; ${ }^{1} \mathrm{H}$ NMR $\left(400 \mathrm{MHz}, \mathrm{CDCl}_{3}\right)(E, E)$-isomer: $\delta 0.88(\mathrm{t}, J=7.0 \mathrm{~Hz}, 6 \mathrm{H}) ; 1.25$ 
$(\mathrm{m}, 32 \mathrm{H}) ; 1.47(\mathrm{~m}, 4 \mathrm{H}) ; 1.98(\mathrm{br}, 1 \mathrm{H}) ; 2.16:(\mathrm{q}, J=7.2,4 \mathrm{H}) ; 2.96(\mathrm{~m}, 4 \mathrm{H}) ; 4.14(\mathrm{q}, J=5.3$ $\mathrm{Hz}, 4 \mathrm{H}) ; 7.39(\mathrm{t}, J=6.5 \mathrm{~Hz}, 2 \mathrm{H}) ;{ }^{13} \mathrm{CNMR}\left(100 \mathrm{MHz}, \mathrm{CDCl}_{3}\right) \delta$ 14.1, 22.6, 25.7, 26.2, 26.7, 29.1, 29.3, 29.4, 29.5, 29.6, 31.9, 48.6, 48.7, 48.8, 72.3, 72.7, 151.5, 152.4.

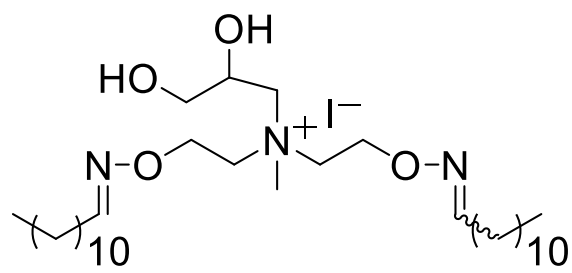

(21E)-17-(2,3-dihydroxypropyl)-17-methyl-14,20-dioxa-13,17,21-triazatritriaconta-12,21dien-17-ium iodide (Lipid 1)

Following a modified version of the procedure for glycidol epoxide opening described by Kuwabe et al., ${ }^{173}$ to a solution of the amine generated in the previous step $(1.09 \mathrm{~g}, 2.33 \mathrm{mmol})$ in 1:1 water/ethanol $(12 \mathrm{~mL})$ was added racemic glycidol $(0.568 \mathrm{~g}$, $7.67 \mathrm{mmol})$ and acetic acid $(0.073 \mathrm{~g}, 1.22 \mathrm{mmol})$. The reaction flask was covered with foil and stirred in the dark at $55^{\circ} \mathrm{C}$ for $48 \mathrm{~h}$. Note: reactions of this type were later performed without acid at $1 \mathrm{M}$ in ethanol (95\%), with 1 eq. glycidol over $12 \mathrm{~h}$ at rt. The ethanol was removed by rotary evaporation and $\mathrm{Et}_{2} \mathrm{O}(50 \mathrm{~mL})$ was added to dissolve the residue. The $\mathrm{Et}_{2} \mathrm{O}$ solution was washed with sat. $\mathrm{NaHCO}_{3}(50 \mathrm{~mL})$. The aqueous wash layer was extracted with $\mathrm{Et}_{2} \mathrm{O}(2 \times 50 \mathrm{~mL})$ and the combined $\mathrm{Et}_{2} \mathrm{O}$ phase was dried $\left(\mathrm{NaSO}_{4}\right)$ and then evaporated by rotary evaporation. The residue was purified by silica column chromatography $\left(\mathrm{CH}_{2} \mathrm{Cl}_{2}: \mathrm{CH}_{3} \mathrm{OH}, 19: 1\right.$ to $\left.9: 1\right)$ to afford 4.5 (1.01 g, 80\%) as a yellow oil; TLC, $\mathrm{R}_{\mathrm{f}}=0.47\left(\mathrm{CH}_{2} \mathrm{Cl}_{2} / \mathrm{MeOH}, 9: 1, p\right.$-anisaldehyde stain); ${ }^{1} \mathrm{H} \mathrm{NMR}\left(400 \mathrm{MHz}, \mathrm{CDCl}_{3}\right)(E, E)-$ isomer: $\delta 0.88(\mathrm{t}, J=6.6 \mathrm{~Hz}, 6 \mathrm{H}) ; 1.26(\mathrm{~m}, 32 \mathrm{H}) ; 1.46(\mathrm{~m}, 4 \mathrm{H}) ; 2.17(\mathrm{q}, J=7.1 \mathrm{~Hz}, 4 \mathrm{H}) ; 2.71$ 
$(\mathrm{m}, 2 \mathrm{H}) ; 2.95(\mathrm{br}, 4 \mathrm{H}) ; 3.48(\mathrm{~m}, 1 \mathrm{H}) ; 3.72(\mathrm{dd}, \mathrm{J}=4.0,11.2 \mathrm{~Hz}, 2 \mathrm{H}) ; 3.88(\mathrm{br}, 1 \mathrm{H}) ; 4.15(\mathrm{~m}$, 4H); $7.38(\mathrm{t}, \mathrm{J}=6.4 \mathrm{~Hz}, 2 \mathrm{H}) ; \mathrm{HRMS} m / z$ calcd $\left[\mathrm{C}_{31} \mathrm{H}_{64} \mathrm{~N}_{3} \mathrm{O}_{4}\right]^{+}[\mathrm{M}+\mathrm{H}]^{+}:$542.48913, observed 542.48952.

A pressure tube was charged with a solution of $4.5(0.85 \mathrm{~g}, 1.57 \mathrm{mmol})$ in $\mathrm{CH}_{2} \mathrm{Cl}_{2}$ (3.5 mL). To the solution was added iodomethane $(2.23 \mathrm{~g}, 15.7 \mathrm{mmol})$. The tube was sealed and then heated at $60^{\circ} \mathrm{C}$ for $6 \mathrm{~h}$. On cooling, the tube was opened and the solvent and excess iodomethane were evaporated using a nitrogen stream in a closed fume hood. The residue was purified by silica column chromatography $\left(\mathrm{CH}_{2} \mathrm{Cl}_{2}: \mathrm{CH}_{3} \mathrm{OH}\right.$ 1:0 to 9:1) to yield lipid 1 (0.941 g, 88\%) as an amber oil; TLC, $\mathrm{R}_{\mathrm{f}}=0.68\left(\mathrm{CH}_{2} \mathrm{Cl}_{2} / \mathrm{MeOH}, 9: 1, p\right.$ anisaldehyde stain); ${ }^{1} \mathrm{H} \mathrm{NMR}\left(400 \mathrm{MHz}, \mathrm{CDCl}_{3}\right)(E, E)$-isomer: $\delta 0.85(\mathrm{t}, J=7.2 \mathrm{~Hz}, 6 \mathrm{H}) ; 1.23$ (m, 30H); $1.44(\mathrm{~m}, 4 \mathrm{H}) ; 2.15(\mathrm{q}, J=7.4 \mathrm{~Hz}, 4 \mathrm{H}) ; 3.37(\mathrm{~s}, 3 \mathrm{H}) ; 3.68(\mathrm{~m}, 3 \mathrm{H}) ; 3.85-4.01(\mathrm{~m}$, $5 \mathrm{H}) ; 4.45(\mathrm{~m}, 5 \mathrm{H}) ; 7.39(\mathrm{t}, J=6.2 \mathrm{~Hz}, 2 \mathrm{H}) ;{ }^{13} \mathrm{C} \mathrm{NMR}\left(100 \mathrm{MHz}, \mathrm{CDCl}_{3}\right) \delta$ 13.9, 16.8, 22.6, 26.0, 26.1, 26.4, 29.1, 29.2, 29.3 (2 signals), 29.4, 29.5, 31.8, 51.6, 62.7, 62.9, 63.1, 64.1, $65.1,66.3,66.9,67.3$ (2 signals), 153.7, 154.3; HRMS $m / z$ calcd $\left[\mathrm{C}_{32} \mathrm{H}_{66} \mathrm{~N}_{3} \mathrm{O}_{4}\right]^{+}: 556.50478$, observed 556.50476. 
<smiles>C/C=N\OCCNCCO/N=C/C</smiles>

16,22-dioxa-15,19,23-triazaheptatriaconta-14,23-diene

Bis-aminooxy carbamate 4.1 (0.105 g, $0.446 \mathrm{mmol})$ was dissolved in $\mathrm{CH}_{2} \mathrm{Cl}_{2}$ (4.0 $\mathrm{mL}$ ). To this solution was added tetradecanal $(0.208 \mathrm{~g}, 0.982 \mathrm{mmol})$, prepared from tetradecanol by Swern oxidation. ${ }^{172}$ After stirring the reaction for $10 \mathrm{~h}$ at $\mathrm{rt}$, the solvent was removed by rotary evaporation and the crude product was purified by silica column chromatography $\left(\mathrm{CH}_{2} \mathrm{Cl}_{2}\right.$ : EtOAc, $1: 0$ to $\left.9: 1\right)$ to afford tert-butyl $N, N$-bis(\{2[(tetradecylideneamino)oxy]ethyl\})carbamate (4.3) $(0.199 \mathrm{~g}, 71 \%)$ as a colorless oil. The mixture of $(E, E):(E, Z)$ diastereomers was used directly in the next step without isomer separation; TLC, $R_{f}$ 0.50, 0.46 (hexanes/EtOAc, 4:1, ninhydrin stain); FT-IR 2954, 2923, 2853, 1698, 1466, 1406, $1366 \mathrm{~cm}^{-1} ;{ }^{1} \mathrm{H}$ NMR (400 MHz, $\left.\mathrm{CDCl}_{3}\right)(E, E)$-isomer (major): $\delta 0.88$ (t, J = 6.6 Hz, 6H); $1.26(\mathrm{~m}, 44 \mathrm{H}), 1.45(\mathrm{~s}, 9 \mathrm{H}) ; 2.16(\mathrm{q}, J=6.9 \mathrm{~Hz}, 4 \mathrm{H}) ; 3.46(\mathrm{~m}, 4 \mathrm{H}) ; 4.11$ $(\mathrm{m}, 4 \mathrm{H}) ; 7.37(\mathrm{t}, J=6.0 \mathrm{~Hz}, 2 \mathrm{H}) ;{ }^{13} \mathrm{C}$ NMR $\left(125 \mathrm{MHz}, \mathrm{CDCl}_{3}\right) \delta 14.1,22.7,25.7,26.2,26.7$, 28.4, 29.1, 29.3, 29.4, 29.5 (2 signals), 29.6 (3 signals), 29.7, 31.9, 47.6, 47.3, 71.7, 72.2, $79.5,151.25,152.2,155.4$.

The bis-oxime ether $4.3(0.467 \mathrm{~g}, 0.748 \mathrm{mmol})$ was dissolved in 1:1 mixture of trifluoro-acetic acid: $\mathrm{CH}_{2} \mathrm{Cl}_{2}(3.2 \mathrm{~mL})$ and the mixture was stirred at $\mathrm{rt}$ for 3 hours. The solvent was evaporated by rotary evaporation and $\mathrm{Et}_{2} \mathrm{O}(30 \mathrm{~mL})$ was added to dissolve the residue. The $\mathrm{Et}_{2} \mathrm{O}$ solution was washed with sat. $\mathrm{NaHCO}_{3}(30 \mathrm{~mL})$. The aqueous wash layer was extracted with $\mathrm{Et}_{2} \mathrm{O}(2 \times 20 \mathrm{~mL})$ and the combined $\mathrm{Et}_{2} \mathrm{O}$ phase was dried $\left(\mathrm{NaSO}_{4}\right)$ 
and then evaporated by rotary evaporation to yield the bis-oxime ether amine $(0.36 \mathrm{~g}$, 91\%) as an off-white solid; TLC, $\mathrm{Rf}_{\mathrm{f}} 0.66\left(\mathrm{CH}_{2} \mathrm{Cl}_{2} / \mathrm{MeOH}, 9: 1\right.$, ninhydrin stain); FT-IR 2955, 2917, 2849, $1463 \mathrm{~cm}^{-1}$; ${ }^{1} \mathrm{H}$ NMR $\left(500 \mathrm{MHz}, \mathrm{CDCl}_{3}\right)(E, E)$-isomer: $\delta 0.88(\mathrm{t}, J=6.7 \mathrm{~Hz}, 6 \mathrm{H})$; $1.25(\mathrm{~m}, 40 \mathrm{H}), 1.47(\mathrm{~m}, 4 \mathrm{H}) ; 1.98(\mathrm{br}, 1 \mathrm{H}) ; 2.16:(\mathrm{q}, J=7.2,4 \mathrm{H}) ; 2.96(\mathrm{~m}, 4 \mathrm{H}) ; 4.14(\mathrm{q}, J=$ $5.3 \mathrm{~Hz}, 4 \mathrm{H}) ; 7.39(\mathrm{t}, J=6.5 \mathrm{~Hz}, 2 \mathrm{H}) ;{ }^{13} \mathrm{C} \mathrm{NMR}\left(100 \mathrm{MHz}, \mathrm{CDCl}_{3}\right) \delta$ 14.3, 22.9, 25.9, 26.4, 26.9, 29.4, 29.6 (2 signals), 29.7 (2 signals), 29.8, 29.9 (2 signals), 32.1, 48.8, 71.8, 152.0. HRMS $m / z$ calcd $\left[\mathrm{C}_{37} \mathrm{H}_{74} \mathrm{~N}_{3} \mathrm{O}_{4}\right]^{+}[\mathrm{M}+\mathrm{H}]^{+}:$624.56738, observed 624.56784 . 


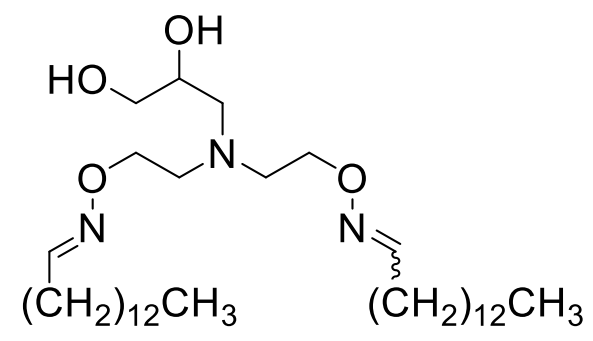

3-(16,22-dioxa-15,19,23-triazaheptatriaconta-14,23-dien-19yl)propane-1,2-diol (13)

Following a modified version of the procedure for glycidol epoxide opening described by Kuwabe et al., ${ }^{173}$ to a solution of the amine prepared in the previous step $(0.166 \mathrm{~g}, 0.317 \mathrm{mmol})$ in ethanol $(0.28 \mathrm{~mL})$ was added racemic glycidol $(0.035 \mathrm{~g}, 0.476$ $\mathrm{mmol})$. The reaction flask was covered with foil and stirred in the dark at $\mathrm{rt}$ for $57 \mathrm{~h}$. The solvent then was removed by rotary evaporation and the residue was purified by silica column chromatography $\left(\mathrm{CH}_{2} \mathrm{Cl}_{2}: \mathrm{CH}_{3} \mathrm{OH}, 1: 0\right.$ to 9:1) to afford bis-oxime ether 4.6 (0.078 g, 41\%) as a light yellow oil; TLC, $\mathrm{R}_{\mathrm{f}}=0.59\left(\mathrm{CH}_{2} \mathrm{Cl}_{2} / \mathrm{MeOH}, 9: 1, p\right.$-anisaldehyde stain); FTIR 3380, 2921, 2854, 1465, 1380, $1041 \mathrm{~cm}^{-1} ;{ }^{1} \mathrm{H}$ NMR (400 MHz, CDCl 3$)(E, E)$-isomer: $\delta 0.88$ $(\mathrm{t}, J=6.6 \mathrm{~Hz}, 6 \mathrm{H}) ; 1.25(\mathrm{~m}, 40 \mathrm{H}), 1.45(\mathrm{sex}, J=6.8 \mathrm{~Hz}, 4 \mathrm{H}) ; 2.17(\mathrm{q}, J=7.1 \mathrm{~Hz}, 4 \mathrm{H}) ; 2.87(\mathrm{~m}$, $2 \mathrm{H}) ; 3.11(\mathrm{br}, 4 \mathrm{H}) ; 3.52(\mathrm{~m}, 1 \mathrm{H}) ; 3.72(\mathrm{dd}, J=3.8,11.4 \mathrm{~Hz}, 1 \mathrm{H}) ; 3.88(\mathrm{br}, 1 \mathrm{H}) ; 4.21(\mathrm{~m}, 4 \mathrm{H})$; $7.39(\mathrm{t}, J=6.4 \mathrm{~Hz}, 2 \mathrm{H}) ;{ }^{13} \mathrm{C} \mathrm{NMR}\left(100 \mathrm{MHz}, \mathrm{CDCl}_{3}\right) \delta 14.3,22.9,26.0,26.4,26.9,29.4,29.6$ (3 signals), 29.7, 29.9 (2 signals), 32.1, 54.1, 54.2, 58.3, 64.6, 67.8, 70.2, 152.4. HRMS m/z calcd $\left[\mathrm{C}_{35} \mathrm{H}_{72} \mathrm{~N}_{3} \mathrm{O}_{4}\right]^{+}[\mathrm{M}+\mathrm{H}]^{+}:$598.5517, observed 598.5517. 


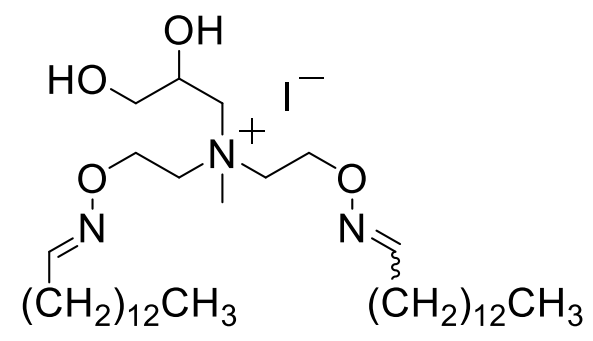

19-(2,3-dihydroxypropyl)-19-methyl-16,22-dioxa-15,19,23-triazaheptatriaconta-14,23-

dien-19-ium iodide (Lipid 2)

[Step $f$ ] A pressure tube was charged with a solution of $4.6(0.078 \mathrm{~g}, 0.131 \mathrm{mmol})$ in $\mathrm{CH}_{2} \mathrm{Cl}_{2}(2.0 \mathrm{~mL})$. To the solution was added iodomethane $(0.372 \mathrm{~g}, 2.62 \mathrm{mmol})$. The tube was sealed and then heated at $55^{\circ} \mathrm{C}$ for $18 \mathrm{~h}$. On cooling, the tube was opened and the solvent and excess iodomethane were evaporated using a nitrogen stream in a closed fume hood. The residue was purified by silica column chromatography $\left(\mathrm{CH}_{2} \mathrm{Cl}_{2}\right.$ : $\mathrm{CH}_{3} \mathrm{OH}$ 9:1) to yield lipid $4(0.062 \mathrm{~g}, 64 \%)$ as a hygroscopic yellow solid; TLC, $R_{f}=0.71$ $\left(\mathrm{CH}_{2} \mathrm{Cl}_{2} / \mathrm{MeOH}, 9: 1\right.$, phosphomolybdic acid stain); FT-IR 3329, 2921, 2854, 1790, 1734, $1702,1466,1368,1271,1186,1056 \mathrm{~cm}^{-1} ;{ }^{1} \mathrm{H}$ NMR $\left(400 \mathrm{MHz}, \mathrm{CDCl}_{3}\right)(E, E)$-isomer: $\delta 0.87$ $(\mathrm{t}, J=6.8 \mathrm{~Hz}, 6 \mathrm{H}) ; 1.25(\mathrm{~m}, 40 \mathrm{H}) ; 1.46(\mathrm{~m}, 4 \mathrm{H}) ; 2.18(\mathrm{q}, J=7.1 \mathrm{~Hz}, 4 \mathrm{H}) ; 3.40(\mathrm{~s}, 3 \mathrm{H}) ; 3.74$ $(\mathrm{m}, 3 \mathrm{H}) ; 3.94(\mathrm{~m}, 6 \mathrm{H}) ; 4.50(\mathrm{~m}, 5 \mathrm{H}) ; 4.65(\mathrm{~m}, 1 \mathrm{H}) ; 7.42(\mathrm{t}, J=6.2 \mathrm{~Hz}, 2 \mathrm{H}) ;{ }^{13} \mathrm{C}$ NMR $(100$ $\mathrm{MHz}, \mathrm{CDCl}_{3}$ ) $\delta 14.3,22.9,26.3$ (2 signals), 26.7, 29.4, 29.6 (3 signals), 29.7, 29.9, 32.1, 51.9, $62.9,64.5,66.3,66.4,67.0,67.5,154.0,154.6 ; \mathrm{HRMS} m / z$ calcd $\left[\mathrm{C}_{36} \mathrm{H}_{74} \mathrm{~N}_{3} \mathrm{O}_{4}\right]+612.5674$, observed 612.5673. 


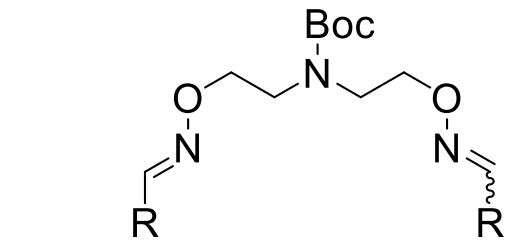

$\mathrm{R}=(\mathrm{Z})-\left(\mathrm{CH}_{2}\right)_{7} \mathrm{CH}=\mathrm{CH}\left(\mathrm{CH}_{2}\right)_{7} \mathrm{CH}_{3}$

Tert-butyl N,N-bis[2-(\{[(9Z)-octadec-9-en-1-ylidene]amino\}oxy)ethyl]carbamate (4.4)

Bis-aminooxy carbamate 4.1 (0.495 g, $2.10 \mathrm{mmol}$ ) was dissolved in $\mathrm{CH}_{2} \mathrm{Cl}_{2}(12 \mathrm{~mL})$.

To this solution was added oleyl aldehyde (1.966 $\mathrm{g}, 8.42 \mathrm{mmol})$, prepared from oleyl alcohol by Swern oxidation. ${ }^{172}$ After stirring the reaction for $12 \mathrm{~h}$ at $\mathrm{rt}$, the solvent was removed by rotary evaporation and the crude product was purified by silica column chromatography $\left(\mathrm{CH}_{2} \mathrm{Cl}_{2}\right.$ : EtOAc, 1:0 to $\left.19: 1\right)$ to afford $4.4(0.731 \mathrm{~g}, 47 \%)$ as a colorless oil. The mixture of $(E, E):(E, Z)$ diastereomers was used directly in the next step without isomer separation; TLC, $\mathrm{R}_{\mathrm{f}}$ 0.32, $0.25\left(\mathrm{CH}_{2} \mathrm{Cl}_{2}\right.$, phosphomolybdic acid stain); FT-IR 3006, 2923, $2855,1700,1462,1407,1366,1150 \mathrm{~cm}^{-1} ;{ }^{1} \mathrm{H}$ NMR (400 MHz, $\left.\mathrm{CDCl}_{3}\right)(E, E)$-isomer (major): $\delta 0.88(\mathrm{t}, J=6.6 \mathrm{~Hz}, 6 \mathrm{H}) ; 1.30(\mathrm{~m}, 48 \mathrm{H}), 1.45(\mathrm{~s}, 9 \mathrm{H}) ; 1.99(\mathrm{~m}, 8 \mathrm{H}) ; 2.16(\mathrm{q}, J=7.2 \mathrm{~Hz}, 4 \mathrm{H})$; $3.47(\mathrm{~m}, 4 \mathrm{H}) ; 4.12(\mathrm{~m}, 4 \mathrm{H}) ; 5.35(\mathrm{~m}, 4 \mathrm{H}) ; 7.37(\mathrm{t}, \mathrm{J}=6.0 \mathrm{~Hz}, 2 \mathrm{H}) ;{ }^{13} \mathrm{C} \mathrm{NMR}\left(125 \mathrm{MHz}, \mathrm{CDCl}_{3}\right)$ 814.1, 22.7, 25.8, 26.3, 26.8, 27.2, 27.3, 28.5, 29.2, 29.2, 29.3, 29.4 (2 signals), 29.6, 29.7 (2 signals), 29.8, 31.9, 32.6, 47.4, 71.8, 72.2, 79.5, 110.1, 129.7, 130.0, 151.2, 152.2, 155.4 . HRMS $m / z$ calcd $\left[\mathrm{C}_{45} \mathrm{H}_{86} \mathrm{~N}_{3} \mathrm{O}_{4}\right]^{+}[\mathrm{M}+\mathrm{H}]^{+}:$732.66128, observed 732.66145 . 


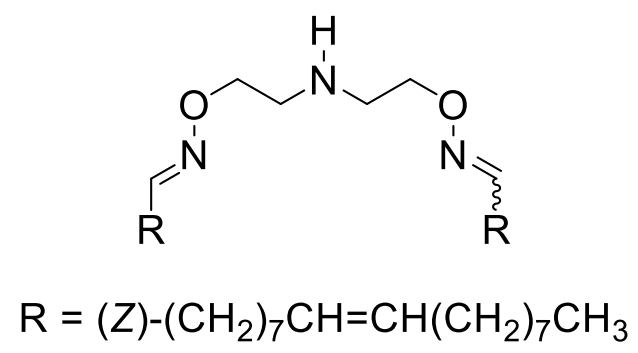

(9Z,36Z)-20,26-dioxa-19,23,27-triazapentatetraconta-9,18,27,36-tetraene

The bis-oxime ether $4.4(0.281 \mathrm{~g}, 0.383 \mathrm{mmol})$ was dissolved in a 1:1 mixture of trifluoro-acetic acid: $\mathrm{CH}_{2} \mathrm{Cl}_{2}(4 \mathrm{~mL})$ and the mixture was stirred at $\mathrm{rt}$ for $1 \mathrm{~h}$. The solvent was evaporated by rotary evaporation and $\mathrm{Et}_{2} \mathrm{O}(60 \mathrm{~mL})$ was added to dissolve the residue. The $\mathrm{Et}_{2} \mathrm{O}$ solution was washed with sat. $\mathrm{NaHCO}_{3}(60 \mathrm{~mL})$. The aqueous wash layer was extracted with $\mathrm{Et}_{2} \mathrm{O}(2 \times 40 \mathrm{~mL})$ and the combined $\mathrm{Et}_{2} \mathrm{O}$ phase was dried $\left(\mathrm{NaSO}_{4}\right)$ and then evaporated by rotary evaporation to yield the bis-oxime ether amine as an amber oil which was used without further purification; TLC, $\mathrm{R}_{f} 0.29\left(\mathrm{CH}_{2} \mathrm{Cl}_{2} / \mathrm{MeOH}, 19: 1\right.$, phosphomolybdic acid stain); FT-IR 3006, 2923, 2855, 1462, $1068 \mathrm{~cm}^{-1} ;{ }^{1} \mathrm{H}$ NMR (400 MHz, $\left.\mathrm{CDCl}_{3}\right)(E, E)$-isomer: $\delta 0.87(\mathrm{t}, J=6.8 \mathrm{~Hz}, 6 \mathrm{H}) ; 1.28(\mathrm{~m}, 44 \mathrm{H}), 1.45(\mathrm{~m}, 4 \mathrm{H}) ; 2.01(\mathrm{~m}, 8 \mathrm{H}) ; 2.16$ : (q, $J=7.2,4 \mathrm{H}) ; 2.49(\mathrm{br}, 1 \mathrm{H}) ; 3.02(\mathrm{~m}, 4 \mathrm{H}) ; 4.16(\mathrm{t}, J=5.4 \mathrm{~Hz}, 4 \mathrm{H}) ; 5.36(\mathrm{~m}, 4 \mathrm{H}) ; 7.39(\mathrm{t}$, $J=6.0 \mathrm{~Hz}, 2 \mathrm{H}) ;{ }^{13} \mathrm{C} \mathrm{NMR}\left(100 \mathrm{MHz}, \mathrm{CDCl}_{3}\right) \delta 14.2,22.8,25.7,26.3,26.8,27.2,27.3,29.2$, 29.3, 29.4, 29.8 (2 signals), 32.0, 32.7, 48.5, 48.7, 71.8, 72.2, 129.8, 130.1, 151.7, 152.6. HRMS $m / z$ calcd $\left[\mathrm{C}_{40} \mathrm{H}_{78} \mathrm{~N}_{3} \mathrm{O}_{2}\right]^{+}[\mathrm{M}+\mathrm{H}]^{+}:$632.60886, observed 632.60907. 


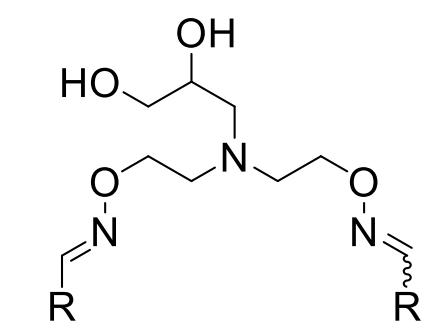

$\mathrm{R}=(\mathrm{Z})-\left(\mathrm{CH}_{2}\right)_{7} \mathrm{CH}=\mathrm{CH}\left(\mathrm{CH}_{2}\right)_{7} \mathrm{CH}_{3}$

3-[(9Z,36Z)-20,26-dioxa-19,23,27-triazapentatetraconta-9,18,23,36-tetraen-

23yl]propane-1,2-diol (4.7)

Following a modified version of the procedure for glycidol epoxide opening described by Kuwabe et al., ${ }^{173}$ to a solution of the amine produced in the previous step $(0.154 \mathrm{~g}, 0.243 \mathrm{mmol})$ in ethanol $(0.25 \mathrm{~mL})$ was added racemic glycidol $(0.020 \mathrm{~g}, 0.268$ mmol). The reaction flask was covered with foil and stirred in the dark at $\mathrm{rt}$ for $16 \mathrm{~h}$. The solvent then was removed by rotary evaporation and the residue was purified by silica column chromatography $\left(\mathrm{CH}_{2} \mathrm{Cl}_{2}: \mathrm{CH}_{3} \mathrm{OH}, 1: 0\right.$ to 9:1) to afford bis-oxime ether 4.7 (0.066 g, 38\%) as a light yellow oil; TLC, $\mathrm{R}_{\mathrm{f}}=0.66\left(\mathrm{CH}_{2} \mathrm{Cl}_{2} / \mathrm{MeOH}, 19: 1\right.$, phosphomolybdic acid stain); FT-IR 3423, 2955, 2923, 2854, 1728, 1462, 1274, 1123, 1072, $738 \mathrm{~cm}^{-1}$; ${ }^{1} \mathrm{H}$ NMR (400 MHz, CDCl $)_{3}(E, E)$-isomer: $\delta 0.87(\mathrm{t}, J=6.6 \mathrm{~Hz}, 6 \mathrm{H}) ; 1.27(\mathrm{~m}, 44 \mathrm{H}), 1.45(\mathrm{~m}, 4 \mathrm{H}) ; 2.00$ (m, 8H); $2.16(\mathrm{q}, \mathrm{J}=7.1 \mathrm{~Hz}, 4 \mathrm{H}) ; 2.65(\mathrm{~m}, 2 \mathrm{H}) ; 2.88 \mathrm{~m}, 4 \mathrm{H}) ; 3.03(\mathrm{br}, 2 \mathrm{H}) ; 3.47(\mathrm{~m}, 1 \mathrm{H}) ; 3.71$ (m, 2H); $4.12(\mathrm{~m}, 4 \mathrm{H}) ; 5.33(\mathrm{~m}, 4 \mathrm{H}) ; 7.37(\mathrm{t}, J=6.2 \mathrm{~Hz}, 2 \mathrm{H}) ;{ }^{13} \mathrm{C} \mathrm{NMR}\left(100 \mathrm{MHz}, \mathrm{CDCl}_{3}\right) \delta$ 14.2, 22.8, 25.9, 26.4, 26.9, 27.3 (2 signals), 29.3, 29.4 (3 signals), 29.5, 29.6, 29.8 (2 signals), 29.9, 32.0, 32.7, 38.8, 54.0, 57.6, 64.5, 68.2, 68.3, 71.1, 71.6 (2 signals), 129.8, 130.1, 151.6, 152.4. HRMS $\mathrm{m} / \mathrm{z}$ calcd $\left[\mathrm{C}_{43} \mathrm{H}_{84} \mathrm{~N}_{3} \mathrm{O}_{4}\right]^{+}[\mathrm{M}+\mathrm{H}]^{+}:$706.64563, observed 706.64573. 


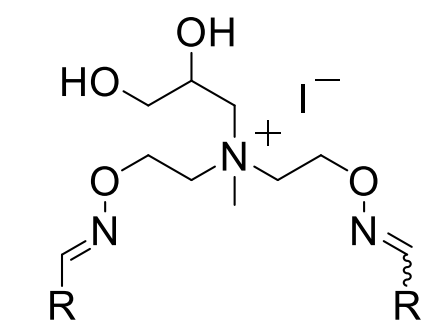

$\mathrm{R}=(\mathrm{Z})-\left(\mathrm{CH}_{2}\right)_{7} \mathrm{CH}=\mathrm{CH}\left(\mathrm{CH}_{2}\right)_{7} \mathrm{CH}_{3}$

(9Z,36Z)-23-(2,3-dihydroxypropyl)-23-methyl-20,26-dioxa-19,23,27-

triazapentatetraconta-9,18,27,36-tetraen-23-ium iodide (Lipid 3)

A pressure tube was charged with a solution of $4.7(0.053 \mathrm{~g}, 0.075 \mathrm{mmol})$ in $\mathrm{CH}_{2} \mathrm{Cl}_{2}$ $(1.0 \mathrm{~mL})$. To the solution was added iodomethane $(0.213 \mathrm{~g}, 1.50 \mathrm{mmol})$. The tube was sealed and then heated at $50^{\circ} \mathrm{C}$ for $6 \mathrm{~h}$. On cooling, the tube was opened and the solvent and excess iodomethane were evaporated using a nitrogen stream in a closed fume hood. The residue was purified by silica column chromatography $\left(\mathrm{CH}_{2} \mathrm{Cl}_{2}: \mathrm{CH}_{3} \mathrm{OH} 19: 1\right.$ to 9:1) to yield lipid $3(0.040 \mathrm{~g}, 63 \%)$ as a yellow semisolid; TLC, $\mathrm{R}_{f} 0.63\left(\mathrm{CH}_{2} \mathrm{Cl}_{2} / \mathrm{MeOH}, 9: 1\right.$, phosphomolybdic acid stain); FT-IR 3459, 3336, 3001, 2969, 2955, 2923, 2855, 1736, $1457,1365,1269,1228,1219,1123,1072,958,742 \mathrm{~cm}^{-1} ;{ }^{1} \mathrm{H}$ NMR $\left(400 \mathrm{MHz}, \mathrm{CDCl}_{3}\right)(E, E)-$ isomer: $\delta 0.86(\mathrm{t}, J=6.4 \mathrm{~Hz}, 6 \mathrm{H}) ; 1.26(\mathrm{~m}, 44 \mathrm{H}) ; 1.45(\mathrm{~m}, 4 \mathrm{H}) ; 1.99(\mathrm{~m}, 8 \mathrm{H}) ; 2.16(\mathrm{q}, J=6.9$ $\mathrm{Hz}, 4 \mathrm{H}) ; 3.39(\mathrm{~s}, 3 \mathrm{H}) ; 3.71(\mathrm{~m}, 3 \mathrm{H}) ; 3.95(\mathrm{~m}, 6 \mathrm{H}) ; 4.50(\mathrm{~m}, 6 \mathrm{H}) ; 5.33(\mathrm{~m}, 4 \mathrm{H}) ; 7.40(\mathrm{t}, J=5.8$ $\left.\mathrm{Hz}, 2 \mathrm{H}) ;{ }^{13} \mathrm{C} \mathrm{NMR} \mathrm{(100} \mathrm{MHz}, \mathrm{CDCl}_{3}\right) \delta 14.2,22.8,26.2$ (2 signals), 26.6, 27.3 (2 signals), 29.2, 29.3 (2 signals), 29.4 (2 signals), 29.6, 29.7, 29.8 (2 signals), 32.0, 32.7, 51.8, 62.7, $62.8,62.9,63.1,64.3,66.0,66.3,66.9,67.4,129.8,130.1,153.8$ 154.5; HRMS $m / z$ calcd $\left[\mathrm{C}_{44} \mathrm{H}_{86} \mathrm{~N}_{3} \mathrm{O}_{4}\right]{ }^{+} 720.66128$, observed 720.66168. 
5.4.2 Synthesis of doxorubicin hexadecanyl acyl hydrazone (Dox-AH)

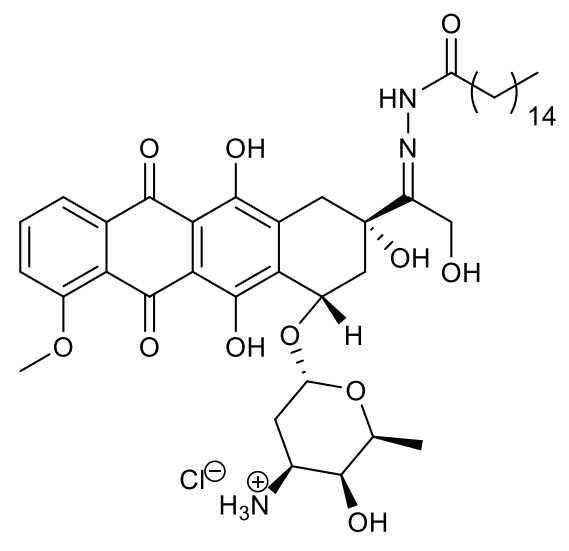

Dox-AH

To doxorubicin hydrochloride $(0.053 \mathrm{~g}, 0.092 \mathrm{mmol})$ in anhydr. $\mathrm{CH}_{3} \mathrm{OH}(10 \mathrm{~mL})$ was added hexadecane hydrazide prepared as in Effenberger et al. ${ }^{152}(0.033 \mathrm{~g}, 0.122 \mathrm{mmol})$, aniline $(0.007 \mathrm{~g}, 0.077 \mathrm{mmol})$, and trifluoroacetic acid $(0.008 \mathrm{~g}, 0.070 \mathrm{mmol})$. The reaction was stirred at $\mathrm{rt}$ for $10 \mathrm{~h}$. The $\mathrm{CH}_{3} \mathrm{OH}$ was removed by rotary evaporation and the product, a red solid, was recrystallized from $\mathrm{CH}_{3} \mathrm{OH}\left(0.065 \mathrm{~g}\right.$, 85\%); ${ }^{1} \mathrm{H} \mathrm{NMR}\left(400 \mathrm{MHz}, \mathrm{CD}_{3} \mathrm{OD}\right) \delta$ $0.89(\mathrm{t}, J=6.6 \mathrm{~Hz}) ; 1.10-1.38(\mathrm{br} \mathrm{m}, 28 \mathrm{H}) ; 1.43(\mathrm{t}, J=5.4 \mathrm{~Hz}, 2 \mathrm{H}) ; 1.67(\mathrm{t}, J=6.8 \mathrm{~Hz}, 1 \mathrm{H})$; 1.84-1.98 (m, 1H); 1.99-2.12 (m, 1H); 2.26-2.45 (m, 3H); $2.55(\mathrm{dd}, J=14.0,6.5 \mathrm{~Hz}, 1 \mathrm{H})$; 2.90 (d, J=17.6 Hz, 1H); 3.16 (br m, 1H); 3.35 (s, 3H); 3.45 (d, J = $17.2 \mathrm{~Hz} 1 \mathrm{H} 3 \mathrm{H}) ; 3.58$ (br $\mathrm{d}, J=12.8 \mathrm{~Hz} 1 \mathrm{H}) ; 3.62-3.71(\mathrm{~m}, 1 \mathrm{H}) ; 4.04(\mathrm{~s}, 3 \mathrm{H}) ; 4.21(\mathrm{dd}, J=8.4 \mathrm{~Hz}, 6.1 \mathrm{~Hz}, 1 \mathrm{H}) ; 4.62(\mathrm{q}$, $J=14.8 \mathrm{~Hz} 2 \mathrm{H}) ; 5.07(\mathrm{t}, J=6.2 \mathrm{~Hz}, 1 \mathrm{H}) ; 5.48(\mathrm{br} \mathrm{s}, 1 \mathrm{H}) ; 7.59(\mathrm{t}, J=9.6 \mathrm{~Hz}, 1 \mathrm{H}), 7.85(\mathrm{t}, J=$ $8.1 \mathrm{~Hz}, 1 \mathrm{H}) ; 7.98(\mathrm{t}, J=10.0 \mathrm{~Hz}, 2 \mathrm{H}) .{ }^{13} \mathrm{C}$ NMR (175 MHz, DMSO-d 6 ) $\delta 186.5$ (2 signals), $186.5,174.0,160.7,156.5,154.2,152.2,136.9,136.3,135.7,134.8,120.0,119.7,119.0$, $110.6,110.5,98.9,72.4,72.1,66.31,66.0,56.6,56.0,46.6,33.9,31.3,31.1, \mathrm{~m}$ 29.1-29.0, 
28.9, 28.8 (2 signals), 28.7, 28.1, 24.1, 22.1, 16.8, 14.0; HRMS $m / z$ calcd $\left[\mathrm{C}_{43} \mathrm{H}_{62} \mathrm{~N}_{3} \mathrm{O}_{11}\right]^{+}$ 796.4379, observed 796.43916.

\subsubsection{Synthesis of core-shell $\mathrm{Fe}_{3} \mathrm{O}_{4}-\mathrm{SiO}_{2} \mathrm{NPs}$}

$\mathrm{SiO}_{2} @ \mathrm{Fe}_{3} \mathrm{O}_{4}$ nanoparticles were prepared using a modified procedure by Pinho et al. ${ }^{127}$ Commercially available $\mathrm{Fe}_{3} \mathrm{O}_{4}$ ferrofluid (Ferrotec EMG 304, $0.25 \mathrm{~mL}$, ca. $58 \mathrm{mg}$ ) dispersed in water $(25 \mathrm{~mL})$ was added in $1 \mathrm{~mL}$ aliquots to a solution of $\mathrm{EtOH}(78 \mathrm{~mL})$ and $\mathrm{NH}_{4} \mathrm{OH}$ (1.55 mL, 28-30\%) under rapid mechanical stirring. Tetraethyl orthosilicate (TEOS) $(0.532 \mathrm{~mL})$ was added to the colloidal suspension and was stirred for $12 \mathrm{~h}$ at $\mathrm{rt}$. The NPs were magnetically sedimented for $1 \mathrm{~h}$ on a neodymium permanent magnet. To collect additional particles, the colloidal supernatant was removed and centrifuged at $6000 \mathrm{rcf}$ for $1 \mathrm{~h}$ at $\mathrm{rt}$. The ethanolic supernatant was removed by aspiration and the combined NP pellets were washed with EtOH $(2 \times 8 \mathrm{~mL})$ with intervening centrifugation (16100 rcf, 20 $\min , \mathrm{rt}$ ) and supernatant removal. After a final wash and centrifugation cycle in water (8 $\mathrm{mL}$ ) with supernatant removal, the NPs were dispersed in water $(4 \mathrm{~mL})$ and freeze-dried resulting in a brown powder (165.4 $\mathrm{mg})$. 


\subsubsection{CML formation and stability}

Lipids 1, 2, and 3 (6.45 mM) in DMSO were each mixed with $1 \mathrm{mg}$ SNP for up to 24h. Dynamic light scattering (DLS) measurements $(0.5 \mathrm{mg} \mathrm{SMP} / \mathrm{mL})$ were made on the mixture at intervals. 3D plots over 24 hour time intervals of the histograms of relative number of particles versus diameter are given in the appendix. In the case of lipids 2 and 3, particles aggregated at mixing times approaching $24 \mathrm{~h}$ to diameters outside a useful range. The following table lists weighted average diameters from size histograms calculated for the DMSO suspension of CML with lipid 1 at $24 \mathrm{~h}$ mixing time and for a water suspension of CML with lipid 1 (after magnetic separation, supernatant removal, and resuspension, $0.25 \mathrm{mg} \mathrm{SNP} / \mathrm{mL})$.

\begin{tabular}{|c|c|c|c|}
\hline Solvent & Diameter $(\mathrm{nm})$ & $\begin{array}{c}\text { Relative } \\
\text { abundance }\end{array}$ & $\begin{array}{c}\text { Weighted avg. } \\
\text { diameter }(\mathrm{nm})\end{array}$ \\
\hline DMSO & 62.62 & 0.59 & 68.2 \\
\hline & 72.89 & 0.29 & \\
\hline & 84.85 & 0.12 & 51.5 \\
\hline Water & 44.56 & 0.29 & \\
\hline & 50.60 & 0.42 & \\
\hline & 57.46 & 0.21 & \\
\hline & 65.24 & 0.08 & \\
\hline
\end{tabular}

DLS measurements of CML with lipid 1 in DMSO and water 


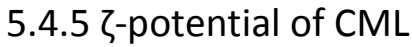

Lipid 1 (6.45 mM) in DMSO was mixed with $1 \mathrm{mg}$ SNP for 5h. CML were magnetically separated, the supernatant was removed, the particles were rinsed with water, and resuspended in water $(0.25 \mathrm{mg} \mathrm{SNP} / \mathrm{mL})$ for $\zeta$-potential measurements (see appendix). The mean $\zeta-$ potential was $31.72+/-2.9 \mathrm{mV}$.

\subsubsection{Calculation of lipid molecules per NP}

The mass of an SNP was calculated based on volume and density according to McCarthy et al. ${ }^{124}$ as $2.073 * 10^{\wedge}-17 \mathrm{~g}$. Volume was estimated based on average SNP diameter from transmission electron microscope images. A typical coating of SNP with lipid used a weight ratio of 0.3-0.4 $\mathrm{mg}$ lipid/mg SNP to achieve the plateau of $\zeta$-potential shown in Figure 4.8 of the manuscript. The number of lipid molecules per SNP that this ratio represents was calculated according to: (moles lipid/g SNP) (mass of 1 SNP) (Avogadro's \#).

\subsubsection{Titration of SNP with cationic lipid 1}

Multiple concentrations of lipid 1 were prepared by serial dilution with water in microcentrifuge tubes. $0.5 \mathrm{~mL}$ of a $0.6 \mathrm{mg} / \mathrm{mL}$ stock solution of SNP in water were added to each tube. Solutions were agitated for several hours. Zeta potentials of the solutions were measured directly after dilution to $0.2 \mathrm{mg} \mathrm{SNP} / \mathrm{mL}$. 


\subsubsection{Estimation of lipid: Dox-AH ratio}

Thin films of lipid 1 and DOX-AH were made by dissolving varying ratios of the two species in $\mathrm{CHCl}_{3} / \mathrm{CH}_{3} \mathrm{OH}$ and removing the solvent in vacuo. To the thin films was added water for a concentration of $2 \mathrm{mM}$ (lipid 1 and DOX-AH combined) and the mixtures were vortex mixed and solicited $10 \mathrm{~min}, 50 \%$ duty, $1 \mathrm{~min}$ intervals. DLS measurements were taken for each solution in water $(2 \mathrm{~mL})$. Figure 4.5 shows the plot of the percent of DOX$\mathrm{AH}$ added versus the diameter of the liposomes: the diameter with the highest relative number of particles from the size histogram.

\subsubsection{Cell toxicity study}

Lipid films were prepared by dissolving lipid, or lipid + DOX-analog mixtures (3:1 molar ratio) in $\mathrm{CH}_{3} \mathrm{OH}$ in microcentrifuge tubes and removing the solvent in vacuo. SNP were dispersed in molecular grade water $(0.185 \mathrm{mg} / \mathrm{mL})$ by briefly sonicating. Aliquots of SNP suspensions were added to the thin films (lipid: $0.108 \mathrm{mM}$, lipid:DOX-analog mixture $0.081: 0.027 \mathrm{mM}$ ) and the mixtures were stirred at $\mathrm{rt} 12 \mathrm{~h}$. The solutions were diluted $10 \%$ with 10x PBS prior to addition to cells.

MCF-7 breast cancer cells were grown in supplemented media (DMEM, 10\% FBS, $1 \%$ Pen strep) in $35 \mathrm{~mm}$ culture dishes. Prior to treatment, cells were plated in 12 -well dishes $(50,000$ cells/well) for biological triplicates and incubated for $24 \mathrm{~h}$. Treatment formulations described above were added directly into cell growth media $(2 \mathrm{~mL}): 0.05 \mathrm{mg}$ SNP, 29.2 total nanomoles lipid or lipid + DOX-analog mixture per well in $0.3 \mathrm{~mL}$ volume. After $2 \mathrm{~h}$ incubation at $37{ }^{\circ} \mathrm{C}$ the medium was removed and replaced with fresh medium. 
Cell viability was assayed after $48 \mathrm{~h}$ incubation by removing media, rinsing $2 \mathrm{X}$ with PBS, adding $0.25 \%$ trypsin-EDTA $1 X(0.2 \mathrm{~mL})$, incubating for 5 minutes, neutralizing with 0.2 $\mathrm{mL}$ DMEM, diluting 1:1 with trypan blue and counting live cells (in replicate for each condition) with a hemocytometer.

\subsubsection{Preparation of oxime-ether liposomes and nucleic acid complexes}

Lipids were solubilized in $\mathrm{CHCl}_{3}$ at a concentration of $10 \mathrm{mg} / \mathrm{mL}$. Lipids (1 mg) were placed in glass vials and then mixed with equimolar amount of the co-lipid DOPE. Lipid films were prepared using rotary evaporatoration under reduced pressure, and kept overnight in a desiccator at room temperature. Lipids films were hydrated with $3 \mathrm{~mL}$ of enzyme free water under vortexing and were probe sonicated for 10 minutes on ice bath with $1 \mathrm{~min}$ cycle of sonication and $1 \mathrm{~min}$ rest to obtain $0.33 \mathrm{mg} / \mathrm{mL}$ of lipid in the liposomes. The resulting OEL liposome preparations were either used for further complexation with nucleic acids (described below), or alternatively, the OEL liposomes were stored at $4^{\circ} \mathrm{C}$ and used within two weeks of their preparation.

To prepare oxime-ether lipid/nucleic acid complexes, the lipids at different concentrations were introduced to fixed concentration of nucleic acids at $500 \mathrm{nM}$ (DS RNAs or RNA/DNA hybrids or DNA duplexes) and incubated for $30 \mathrm{~min}$ at room temperature. After incubation, the mixture was diluted 10 fold by using either enzyme free water/buffer or serum media at room temperature. The final concentration of nucleic acids was $50 \mathrm{nM}$ and the lipid concentrations were typically in the range of 1-20 $\mu \mathrm{M}$. These preparations were then used immediately. 
5.4.11 Cell culture for gene transfection studies

MDA-MB-231 (human breast cancer) cells either stably expressing enhanced green fluorescent protein (GFP) or non-GFP cells were maintained in a Dulbecco's modified Eagle's medium (DMEM) 10\% (v/v) heat-inactivated FBS (fetal bovine serum), 100 i.u./mL penicillin and $100 \mu \mathrm{g} / \mathrm{mL}$ streptomycin under a humidified $5 \% \mathrm{CO}_{2}$ incubator at $37^{\circ} \mathrm{C}$. On the basis of the requirement of the experiments either GFP expressing or nonGFP MDA-MB-231 cells were used.

5.4.12 Binding affinity of oxime ether lipids with hybrid DNA/RNA

Binding affinities of lipids 1-3 with nucleic acids were determined by conducting fluorescent anisotropy/polarization measurements using Tecan Infinite M1000 (Tecan, USA). Fluorescent Alexa-488 labeled RNA/DNA hybrid duplexes (200 nM) were incubated with various concentrations of the lipids. Changes in fluorescent (anisotropy/polarization) values of hybrid duplexes upon binding to the lipids with respect to the hybrids alone was measured at $\lambda_{\mathrm{ex}} 470 \mathrm{~nm}$ and $\lambda_{\mathrm{em}} 517 \mathrm{~nm}$. 


\subsubsection{Cell viability for gene transfection studies}

Viability of the MDA-MB-231 human breast cancer cells was determined on treatment with lipids alone and in complexation with DS RNAs. 20,000 cells/well were seeded in 96 well plates in serum containing media 24 hours prior to experiments. Lipids at different concentrations with and without DS RNAs (50 nM final) were added to the cells in triplicate in serum containing media and incubated for 4 hours at $37^{\circ} \mathrm{C}$. After incubation, the media was replaced with fresh serum-containing media. The cells were then incubated for another $24 \mathrm{~h}$ at $37^{\circ} \mathrm{C}$. At the end of incubation, cell titer blue reagent was added to each well according to the manufacturer's protocol and the cells were further incubated for 4 hours at $37^{\circ} \mathrm{C}$. The fluorescence of the resofurin (converted from resazurin by viable cells) was measured at $\lambda_{\mathrm{ex}} 560 \mathrm{~nm}$ and $\lambda_{\mathrm{em}} 590 \mathrm{~nm}$ with an auto cutoff in a fluorescent ELISA plate reader (Spectra MAX, Molecular Devices, Sunnyvale, CA).

\subsubsection{Transfection and GFP silencing assay}

One day before the experiments 60,000 cells/well of MDA-MB-231 cells and 30,000 cells/well of MDA-MB-231/GFP were plated in two separate 24 well plates for uptake and silencing experiments in serum containing media. On the day of transfection, liposomes complexed with either Alexa-488 RNA/DNA hybrid duplexes or dicer substrates of RNA duplexes (DS RNAs) were added to the cells in serum containing media. The cells were then incubated for $4 \mathrm{~h}$ at $37^{\circ} \mathrm{C}$. After $4 \mathrm{~h}$, the media was replaced with the fresh serum media. Uptake efficiency of RNA/DNA hybrid duplexes and silencing of GFP was 
quantified using Cell Quest software after 1 day and 3 days of transfections respectively by fluorescence-activated cell sorting (FACS).

Approximately 72 hours after the transfection, MDA-MB-231/GFP were imaged with a Nikon 200 TE inverted microscope (Melville, NJ) using Meta Morph software (Universal Imaging Co., Downingtown, PA) to determine the silencing efficiency of GFP by liposome/ DS RNAs complexes. PanFluor 20X, ELWD, NA=0.45 objective and a Nikon B2E/C, 465-495/505/515-555 cube (Chroma Technology Corp., Rockingham, VT) were used for GFP imaging. 


\section{REFERENCES}

1. Kolb, H. C.; Sharpless, K. B. The growing impact of click chemistry on drug discovery. Drug Discovery Today 2003, 8 (24), 1128-1137.

2. Kalia, J.; Raines, R. Hydrolytic stability of hydrazones and oximes. Angewandte Chemie (International ed. in English) 2008, 47 (39), 7523-7526.

3. Ciula, J. C.; Streitwieser, A. Carbon acidity. 79. Acidity of enolate equivalent compounds: oxime ethers. J. Org. Chem. 1991, 56, 1989-93.

4. Selvi, R. S.; Nanthini, R.; Sukanyaa, G. The Basic Principle of Phase-Transfer Catalysis, Some Mechanistic Aspects and Important Applications. International Journal of Scientific and Technology Research 2012, 1 (3), 61-63.

5. $\quad$ Tros de Ilarduya, C.; Sun, Y.; Düzgüneş, N. Gene delivery by lipoplexes and polyplexes. European journal of pharmaceutical sciences : official journal of the European Federation for Pharmaceutical Sciences 2010, 40 (3), 159-170.

6. Zajdel, A.; Wilczok, A.; Slowinski, J.; Orchel, J.; Mazurek, U. Aldehydic lipid peroxidation products in human brain astrocytomas. J Neurooncol 2007, 84, 167-173.

7. Noda, Y.; Berlett, B.; Stadtman, E.; Aponte, A.; Morgan, M.; Shen, R.-F. Identification of enzymes and regulatory proteins in Escherichia coli that are oxidized under nitrogen, carbon, or phosphate starvation. Proceedings of the National Academy of Sciences of the United States of America 2007, 104 (47), 18456-18460.

8. Negre-Salvayre, A.; Auge, N.; Ayala, V.; Basaga, H.; Boada, J.; Brenke, R.; Chapple, S.; Cohen, G.; Feher, J.; Grune, T.; Lengyel, G.; Mann, G.; Pamplona, R.; Poli, G.; Portero-Otin, M.; Riahi, Y.; Salvayre, R.; Sasson, S.; Serrano, J.; Shamni, O.; Siems, W.; Siow, R.; Wiswedel, I.; Zarkovic, K.; Zarkovic, N. Pathological aspects of lipid peroxidation. Free Radical Research 2010, 44 (10), 1125-1171.

9. $\quad$ Griffiths, W.; Koal, T.; Wang, Y.; Kohl, M.; Enot, D.; Deigner, H.-P. Targeted metabolomics for biomarker discovery. Angewandte Chemie (International ed. in English) 2010, 49 (32), 5426-5445.

10. Wikoff, W.; Anfora, A.; Liu, J.; Schultz, P.; Lesley, S.; Peters, E.; Siuzdak, G. Metabolomics analysis reveals large effects of gut microflora on mammalian blood metabolites. Proceedings of the National Academy of Sciences of the United States of America 2009, 106 (10), 3698-3703.

11. Lane, A.; Fan, T.; Xie, Z.; Moseley, H.; Higashi, R. Isotopomer analysis of lipid biosynthesis by high resolution mass spectrometry and NMR. Analytica Chimica Acta 2009, 651 (2), 201-208.

12. Eggink, M.; Wijtmans, M.; Kretschmer, A.; Kool, J.; Lingeman, H.; Esch, I. J. P. d.; Niessen, W. M. A.; Irth, H. Targeted LC-MS derivatization for aldehydes and carboxylic acids with a new derivatization agent 4-APEBA. Anal Bioanal Chem 2010, 397, 665-675.

13. Jacobo, I.; José Manuel, G.; Isabel, M. Determination of carbonyl compounds in fish species samples with solid-phase microextraction with on-fibre derivatization. Food Chemistry 2010, 123.

14. Maboudou, P.; Mathieu, D.; Bachelet, H.; Wiart, J. F.; Lhermitte, M. Detection of oxidative stress. Interest of GC-MS for malondialdehyde and formaldehyde monitoring. Biomedical Chromatography 2002, 16, 199-202.

15. Sugaya, N.; Sakurai, K.; Nakagawa, T.; Onda, N.; Onodera, S.; Morita, M.; Tezuka, M. Development of a headspace $\mathrm{GC} / \mathrm{MS}$ analysis for carbonyl compounds (aldehydes and ketones) in household products after derivatization with o-(2,3,4,5,6-pentafluorobenzyl)hydroxylamine. Analytical sciences : the international journal of the Japan Society for Analytical Chemistry 2004, $20(5), 865-870$. 
16. Barry, S. J.; Carr, R. M.; Lane, S. J.; Leavens, W. J.; Manning, C. O.; Monte, S.;

Waterhouse, I. Use of S-pentafluorophenyl tris(2,4,6-trimethoxyphenyl) phosphonium acetate bromide and (4-hydrazino-4-oxobutyl) tris(2,4,6-trimethoxyphenyl)phosphonium bromide for the derivatization of alcohols, aldehydes and ketones for detection by liquid chromatography/electrospray mass spectrometry. Rapid Commun. Mass Spectrom. 2003, 17 (5), 484-497.

17. Wheeler, O. H. The Girard Reagents. Journal of Chemical Education 1968, 45 (6), 435-

437.

18. Griffiths, W.; Hornshaw, M.; Woffendin, G.; Baker, S.; Lockhart, A.; Heidelberger, S.; Gustafsson, M.; Sjövall, J.; Wang, Y. Discovering oxysterols in plasma: a window on the metabolome. Journal of Proteome Research 2008, 7 (8), 3602-3612.

19. Hong, H.; Wang, Y. Derivatization with Girard reagent T combined with LC-MS/MS for the sensitive detection of 5-formyl-2'-deoxyuridine in cellular DNA. Analytical Chemistry 2007, 79 (1), 322-326.

20. Johnson, D. A modified Girard derivatizing reagent for universal profiling and trace analysis of aldehydes and ketones by electrospray ionization tandem mass spectrometry. Rapid communications in mass spectrometry: RCM 2007, 21 (18), 2926-2932.

21. Iglesias, J.; Gallardo, J. M.; Medina, I. Determination of carbonyl compounds in fish species samples with solid-phase microextraction with on-fibre derivatization. Food Chemistry 2010, 123, 771-778.

22. Sugaya, N.; Sakurai, K.; Nakagawa, T.; Onda, N.; Onodera, S.; Morita, M.; Tezuka, M. Development of a Headspace GC/MS Analysis for Carbonyl Compounds (Aldehydes and Ketones) in Household Products after Derivatization with o-(2,3,4,5,6-Pentafluorobenzyl)-hydroxylamine. Analytical Sciences 2004, 20, 865-870.

23. Fang, K.; Pan, X.; Huang, B.; Liu, J.; Wang, Y.; Gao, J. Simultaneous Derivatization of Hydroxyl and Ketone Groups for the Analysis of Steroid Hormones by GC-MS. Chromatographia 2010, 72, 949-956.

24. Eggink, M.; Wijtmans, M.; Kretschmer, A.; Kool, J.; Lingeman, H.; de Esch, I.; Niessen, W.; Irth, H. Targeted LC-MS derivatization for aldehydes and carboxylic acids with a new derivatization agent 4-APEBA. Analytical and bioanalytical chemistry 2010, 397 (2), 665-675.

25. Hong, H.; Wang, Y. Derivatization with Girard Reagent T Combined with LC-MS/MS for the Sensitive Detection of 5-Formyl-2'-deoxyuridine in Cellular DNA. Anal. Chem. 2007, 79, 322326.

26. Ross, M. M.; Kidwell, D. A.; Colton, R. J. Selective detection of aldehydes and ketones by derivatization/secondary ion mass spectrometry International Journal of Mass Spectrometry and Ion Processes 1985, 63, 141-148.

27. Barry, S. J.; Carr, R. M.; Lane, S. J.; Leavens, W. J.; Manning, C. O.; Monté, S.; Waterhouse, I. Use of S-pentafluorophenyl tris(2,4,6-trimethoxyphenyl)phosphonium acetate bromide and (4-hydrazino-4-oxobutyl) [tris(2,4,6-trimethoxyphenyl)phosphonium bromide for the derivatization of alcohols, aldehydes and ketones for detection by liquid chromatography/electrospray mass spectrometry. Rapid Communications in Mass Spectrometry 2003, 17, 484-497.

28. Griffiths, W. J.; Hornshaw, M.; Woffendin, G.; Baker, S. F.; Lockhart, A.; Heidelberger, S.; Gustafsson, M.; Sjövall, J.; Wang, Y. Discovering Oxysterols in Plasma: A Window on the Metabolome. Journal of Proteome Research 2008, 7, 3602-3612.

29. Johnson, D. W. A modified Girard derivatizing reagent for universal profiling and trace analysis of aldehydes and ketones by electrospray ionization tandem mass spectrometry. Rapid Communications in Mass Spectrometry 2007, 21, 2926-2932. 
30. Yuan, W.; Edwards, J. L.; Li, S. Global profiling of carbonyl metabolites with a photocleavable isobaric labeling affinity tag. Chem. Commun. (Cambridge, U. K.) 2013, 49 (94), 1108011082.

31. Bulte, J. W. M.; Kraitchman, D. L. Iron oxide MR contrast agents for molecular and cellular imaging. NMR in Biomedicine 2004, 17, 484-499.

32. Ma, H.-L.; Qi, X.-R.; Ding, W.-X.; Maitani, Y.; Nagai, T. Magnetic targeting after femoral artery administration and biocompatibility assessment of superparamagnetic iron oxide nanoparticles. Journal of biomedical materials research. Part A 2008, 84 (3), 598-606.

33. Thiesen, B.; Jordan, A. Clinical applications of magnetic nanoparticles for hyperthermia. International journal of hyperthermia : the official journal of European Society for Hyperthermic Oncology, North American Hyperthermia Group 2008, 24 (6), 467-474.

34. Bothun, G.; Lelis, A.; Chen, Y.; Scully, K.; Anderson, L.; Stoner, M. Multicomponent folate-targeted magnetoliposomes: design, characterization, and cellular uptake. Nanomedicine: nanotechnology, biology, and medicine 2011, 7 (6), 797-805.

35. Reddy, L. H.; Arias, J. L.; Nicolas, J.; Couvreur, P. Magnetic Nanoparticles: Design and Characterization, Toxicity and Biocompatibility, Pharmaceutical and Biomedical Applications. Chem. Rev. (Washington, DC, U. S.) 2012, 112 (11), 5818-5878.

36. Massart, R. Preparation of aqueous magnetic liquids in alkaline and acidic media. IEEE Trans. Magn. 1981, MAG-17, 1247-8.

37. Lu, A.-H.; Salabas, E.; Schüth, F. Magnetic nanoparticles: synthesis, protection, functionalization, and application. Angewandte Chemie (International ed. in English) 2007, 46 (8), 1222-1244.

38. Laurent, S.; Forge, D.; Port, M.; Roch, A.; Robic, C.; Vander Elst, L.; Muller, R. N. Magnetic Iron Oxide Nanoparticles: Synthesis, Stabilization, Vectorization, Physicochemical Characterizations, and Biological Applications. Chem. Rev. (Washington, DC, U. S.) 2008, 108 (6), 2064-2110.

39. Sun, C.; Lee, J.; Zhang, M. Magnetic nanoparticles in MR imaging and drug delivery. Advanced drug delivery reviews 2008, 60 (11), 1252-1265.

40. Hasany, S. F.; Ahmed, I.; Rajan, J.; Rehman, A. Systematic review of the preparation techniques of iron oxide magnetic nanoparticles. Nanosci. Nanotechnol. (Rosemead, CA, U. S.) 2012, 2 (6), 148-158, $11 \mathrm{pp.}$

41. Yang, C.; Wu, J.; Hou, Y. Fe3O4 nanostructures: synthesis, growth mechanism, properties and applications. Chem. Commun. (Cambridge, U. K.) 2011, 47, 5130-5141.

42. Salabas, E.-L. Structural and magnetic investigations of magnetic nanoparticles and coreshell colloids. 2005.

43. Yuen, A.; Hutton, G.; Masters, A.; Maschmeyer, T. The interplay of catechol ligands with nanoparticulate iron oxides. Dalton transactions (Cambridge, England : 2003) 2012, 41 (9), 25452559.

44. Lin, M. M.; Kim, H.-H.; Kim, H.; Muhammed, M.; Kim, D. K. Iron oxide-based nanomagnets in nanomedicine: fabrication and applications. Nano Rev. 2010, 1, No pp. given. 45. Mojica Pisciotti, M. L.; Lima, E., Jr.; Vasquez Mansilla, M.; Tognoli, V. E.; Troiani, H. E.; Pasa, A. A.; Creczynski-Pasa, T. B.; Silva, A. H.; Gurman, P.; Colombo, L.; Goya, G. F.; Lamagna, A.; Zysler, R. D. In vitro and in vivo experiments with iron oxide nanoparticles functionalized with DEXTRAN or polyethylene glycol for medical applications: Magnetic targeting. J. Biomed. Mater. Res., Part B 2014, 102B (4), 860-868.

46. Yang, Y.; Jiang, J.-S.; Du, B.; Gan, Z.-F.; Qian, M.; Zhang, P. Preparation and properties of a novel drug delivery system with both magnetic and biomolecular targeting. J. Mater. Sci.: Mater. Med. 2009, 20 (1), 301-307. 
47. Ma, H.-L.; Qi, X.-R.; Ding, W.-X.; Maitani, Y.; Nagai, T. Magnetic targeting after femoral artery administration and biocompatibility assessment of superparamagnetic iron oxide nanoparticles. Journal of Biomedical Materials Research Part A 2007, 598-606.

48. Weissleder, R.; Stark, D. D.; Engelstad, B. L.; Bacon, B. R.; Compton, C. C.; White, D. L.; Jacobs, P.; Lewis, J. Superparamagnetic iron oxide: pharmacokinetics and toxicity. AJR Am J Roentgenol 1989, 152 (1), 167-73.

49. Li, C. H.; Hodgins, P.; Peterson, G. P. Experimental study of fundamental mechanisms in inductive heating of ferromagnetic nanoparticles suspension $\left(\mathrm{Fe}_{3} \mathrm{O}_{4}\right.$ Iron Oxide Ferrofluid) Journal of Applied Physics 2011, 110 (05).

50. Torres-Lugo, M.; Rinaldi, C. Thermal potentiation of chemotherapy by magnetic nanoparticles. Nanomedicine (London, U. K.) 2013, 8, 1689-1707.

51. Riedinger, A.; Guardia, P.; Curcio, A.; Garcia, M. A.; Cingolani, R.; Manna, L.; Pellegrino, T. Subnanometer local temperature probing and remotely controlled drug release based on azofunctionalized iron oxide nanoparticles. Nano Lett. 2013, 13, 2399-2406.

52. Müller, S. Magnetic fluid hyperthermia therapy for malignant brain tumors--an ethical discussion. Nanomedicine : nanotechnology, biology, and medicine 2009, 5 (4), 387-393.

53. Johannsen, M.; Thiesen, B.; Wust, P.; Jordan, A. Magnetic nanoparticle hyperthermia for prostate cancer. International journal of hyperthermia : the official journal of European Society for Hyperthermic Oncology, North American Hyperthermia Group 2010, 26 (8), 790-795.

54. Shultz, M.; Reveles, J.; Khanna, S.; Carpenter, E. Reactive nature of dopamine as a surface functionalization agent in iron oxide nanoparticles. Journal of the American Chemical Society 2007, 129 (9), 2482-2487.

55. Jain, T.; Morales, M.; Sahoo, S.; Leslie-Pelecky, D.; Labhasetwar, V. Iron oxide nanoparticles for sustained delivery of anticancer agents. Molecular pharmaceutics 2005, 2 (3), 194-205.

56. Jing, J.; Zhang, Y.; Liang, J.; Zhang, Q.; Bryant, E.; Avendano, C.; Colvin, V. L.; Wang, Y.; Li, W.; $\mathrm{Yu}, \mathrm{W}$. W. One-step reverse precipitation synthesis of water-dispersible superparamagnetic magnetite nanoparticles. J. Nanopart. Res. 2012, 14 (4), 827/1-827/8.

57. Chen, S. Polymer-coated iron oxide nanoparticles for medical imaging. 2010.

58. Petri-Fink, A.; Steitz, B.; Finka, A.; Salaklang, J.; Hofmann, H. Effect of cell media on polymer coated superparamagnetic iron oxide nanoparticles (SPIONs): colloidal stability, cytotoxicity, and cellular uptake studies. European journal of pharmaceutics and biopharmaceutics : official journal of Arbeitsgemeinschaft für Pharmazeutische Verfahrenstechnik e.V 2008, 68 (1), 129-137.

59. Jung, C. W. Surface properties of superparamagnet iron oxide MR contrast agents: Ferumoxides, Ferumoxtran, Ferumoxsil. Magnetic Resonance Imaging 1995, 13 (5), 675-691. 60. Hidenori, O.; Yukio, N.; Kazunori, K. PEGylated nanoparticles for biological and pharmaceutical applications. Advanced Drug Delivery Reviews 2003, 55.

61. Sun, Q.; Reddy, B. V.; Marquez, M.; Jena, P.; Gonzalez, C.; Wang, Q. Theoretical Study on Gold-Coated Iron Oxide Nanostructure: Magnetism and Bioselectivity for Amino Acids. Journal of Physical Chemistry C 2007, 111.

62. Pope-Harman, A.; Cheng, M. M.-C.; Robertson, F.; Sakamoto, J.; Ferrari, M. Biomedical nanotechnology for cancer. Med. Clin. North Am. 2007, 91 (5), 899-927.

63. Rahman, A. M.; Yusuf, S. W.; Ewer, M. S. Anthracycline-induced cardiotoxicity and the cardiac-sparing effect of liposomal formulation. International Journal of Nanomedicine 2007, 2 (4), 567-583. 
64. Gewirtz, D. A critical evaluation of the mechanisms of action proposed for the antitumor effects of the anthracycline antibiotics adriamycin and daunorubicin. Biochemical pharmacology 1999, 57 (7), 727-741.

65. Fornari, F. A.; Randolph, J. K.; Yalowich, J. C.; Ritke, M. K.; Gewirtz, D. A. Interference by Doxorubicin with DNA Unwinding in MCF-7 Breast Tumor Cells. Molecular Pharmacology 1994, 45, 649-656.

66. Pastorino, F.; Marimpietri, D.; Brignole, C.; Paolo, D. D.; Pagnan, G.; Daga, A.; Piccardi, F.; Cilli, M.; Allen, T. M.; Ponzoni, M. Ligand-Targeted Liposomal Therapies of Neuroblastoma. Current Medicinal Chemistry 2007, 14, 3070-3078.

67. Tian Hu, S.; Brändle, E.; Zbinden, G. Inhibition of Cardiotoxic, Nephrotoxic and Neurotoxic Effects of Doxorubicin by ICRF-159. Pharmacology 1983, 26 (4), 210-220.

68. Carvalho, F. S.; Burgeiro, A.; Garcia, R.; Moreno, A. J.; Carvalho, R. A.; Oliveira, P. J. Doxorubicin-Induced Cardiotoxicity: From Bioenergetic Failure and Cell Death to Cardiomyopathy. Medicinal Research Reviews 2014, 34 (1), 106-135.

69. Hua, M.-Y.; Yang, H.-W.; Liu, H.-L.; Tsai, R.-Y.; Pang, S.-T.; Chuang, K.-L.; Chang, Y.-S.;

Hwang, T.-L.; Chang, Y.-H.; Chuang, H.-C.; Chuang, C.-K. Superhigh-magnetization nanocarrier as a doxorubicin delivery platform for magnetic targeting therapy. Biomaterials 2011, 32 (34), 8999-9010.

70. Li, S.-Z.; Ma, Y.; Yue, X.-L.; Cao, Z.; Dai, Z.-F. One-pot construction of doxorubicin conjugated magnetic silica nanoparticles. New J. Chem. 2009, 33 (12), 2414-2418.

71. Tannock, I. F.; Rotin, D. Acid pH in tumors and its potential for therapeutic exploitation. Cancer Res 1989, 49 (16), 4373-84.

72. Chang, Y.-L.; Meng, X.-L.; Zhao, Y.-L.; Li, K.; Zhao, B.; Zhu, M.; Li, Y.-P.; Chen, X.-S.; Wang, J.-Y. Novel water-soluble and $\mathrm{pH}$-responsive anticancer drug nanocarriers: Doxorubicin-PAMAM dendrimer conjugates attached to superparamagnetic iron oxide nanoparticles (IONPS). J. Colloid Interface Sci. 2011, 363 (1), 403-409.

73. Patra, H. K.; Ul Khaliq, N.; Romu, T.; Wiechec, E.; Borga, M.; Turner, A. P. F.; Tiwari, A. MRI-Visual Order-Disorder Micellar Nanostructures for Smart Cancer Theranostics. Adv. Healthcare Mater. 2014, 3 (4), 526-535.

74. El-Dakdouki, M. H.; Zhu, D. C.; El-Boubbou, K.; Kamat, M.; Chen, J.; Li, W.; Huang, X. Development of Multifunctional Hyaluronan-Coated Nanoparticles for Imaging and Drug Delivery to Cancer Cells. Biomacromolecules 2012, 13 (4), 1144-1151.

75. Gautier, J.; Munnier, E.; Paillard, A.; Herve, K.; Douziech-Eyrolles, L.; Souce, M.; Dubois, P.; Chourpa, I. A pharmaceutical study of doxorubicin-loaded PEGylated nanoparticles for magnetic drug targeting. Int. J. Pharm. (Amsterdam, Neth.) 2012, 423 (1), 16-25.

76. Yolles, S.; Leafe, T. D.; Meyer, F. J. Timed-release depot for anticancer agents. J. Pharm. Sci. 1975, 64 (1), 115-16.

77. Chen, J.; Shi, M.; Liu, P.; Ko, A.; Zhong, W.; Liao, W.; Xing, M. M. Q. Reducible polyamidoamine-magnetic iron oxide self-assembled nanoparticles for doxorubicin delivery. Biomaterials 2014, 35 (4), 1240-1248.

78. Lubbe, A. S.; Bergemann, C.; Riess, H.; Schriever, F.; Reichardt, P.; Possinger, K.; Matthias, M.; Dorken, B.; Herrmann, F.; Gurtler, R.; Hohenberger, P.; Haas, N.; Sohr, R.; Sander, B.; Lemke, A. J.; Ohlendorf, D.; Huhnt, W.; Huhn, D. Clinical experiences with magnetic drug targeting: a phase I study with 4'-epidoxorubicin in 14 patients with advanced solid tumors. Cancer Res 1996, 56 (20), 4686-93.

79. Lee, J. E.; Lee, N.; Kim, H.; Kim, J.; Choi, S. H.; Kim, J. H.; Kim, T.; Song, I. C.; Park, S. P.; Moon, W. K.; Hyeon, T. Uniform Mesoporous Dye-Doped Silica Nanoparticles Decorated with 
Multiple Magnetite Nanocrystals for Simultaneous Enhanced Magnetic Resonance Imaging, Fluorescence Imaging, and Drug Delivery. J. Am. Chem. Soc. 2010, 132 (2), 552-557.

80. Li, W. M.; Chen, S. Y.; Liu, D. M. In situ doxorubicin-CaP shell formation on amphiphilic gelatin-iron oxide core as a multifunctional drug delivery system with improved cytocompatibility, pH-responsive drug release and MR imaging. Acta Biomater. 2013, 9 (2), 5360-5368.

81. Fan, C.-H.; Ting, C.-Y.; Lin, H.-J.; Wang, C.-H.; Liu, H.-L.; Yen, T.-C.; Yeh, C.-K. SPIOconjugated, doxorubicin-loaded microbubbles for concurrent MRI and focused-ultrasound enhanced brain-tumor drug delivery. Biomaterials 2013, 34 (14), 3706-3715.

82. Brulé, S.; Levy, M.; Wilhelm, C.; Letourneur, D.; Gazeau, F.; Ménager, C.; Le Visage, C. Doxorubicin release triggered by alginate embedded magnetic nanoheaters: a combined therapy. Advanced materials (Deerfield Beach, Fla.) 2011, 23 (6), 787-790.

83. Peiris, P. M.; Bauer, L.; Toy, R.; Tran, E.; Pansky, J.; Doolittle, E.; Schmidt, E.; Hayden, E.; Mayer, A.; Keri, R. A.; Griswold, M. A.; Karathanasis, E. Enhanced Delivery of Chemotherapy to Tumors Using a Multicomponent Nanochain with Radio-Frequency-Tunable Drug Release. ACS Nano 2012, 6 (5), 4157-4168.

84. Grochowski, E.; Jurczak, J. A New Synthesis of O-Alkylhydroxylamines. Synthesis 1976, (10), 682-684.

85. Sandler, S. R.; Karo, W. Organic Functional Group Preparations; 2 ed.; Academic Press, Inc.: San Diego, California, 1989; Vol. 3.

86. Li, Z.; Tang, Y.; Liu, Y.; Li, Y. Salting effect in partially miscible systems of n-butanol-water and butanone-water. Fluid Phase Equilibria 1995, 103, 143-153.

87. Yang, W.-C.; Regnier, F.; Jiang, Q.; Adamec, J. In vitro stable isotope labeling for discovery of novel metabolites by liquid chromatography-mass spectrometry: Confirmation of gamma-tocopherol metabolism in human A549 cell. Journal of chromatography. A 2010, 1217 (5), 667-675.

88. Bantscheff, M.; Schirle, M.; Sweetman, G.; Rick, J.; Kuster, B. Quantitative mass spectrometry in proteomics: a critical review. Anal. Bioanal. Chem. 2007, 389 (4), 1017-1031. 89. Psychogios, N.; Hau, D. D.; Peng, J.; Guo, A. C.; Mandal, R.; Bouatra, S.; Sinelnikov, I.; Krishnamurthy, R.; Eisner, R.; Gautam, B.; Young, N.; Xia, J.; Knox, C.; Dong, E.; Huang, P.; Hollander, Z.; Pedersen, T. L.; Smith, S. R.; Bamforth, F.; Greiner, R.; McManus, B.; Newman, J. W.; Goodfriend, T.; Wishart, D. S. The Human Serum Metabolome. PLoS ONE 2011, 6 (2). 90. Tai, L.-A.; Tsai, P.-J.; Wang, Y.-C.; Wang, Y.-J.; Lo, L.-W.; Yang, C.-S. Thermosensitive liposomes entrapping iron oxide nanoparticles for controllable drug release. Nanotechnology 2009, 20 (13), 135101.

91. Qiu, D.; An, X. Controllable release from magnetoliposomes by magnetic stimulation and thermal stimulation. Colloids Surf., B 2013, 104, 326-329.

92. Bringas, E.; Koeysueren, O.; Quach, D. V.; Mahmoudi, M.; Aznar, E.; Roehling, J. D.; Marcos, M. D.; Martinez-Manez, R.; Stroeve, P. Triggered release in lipid bilayer-capped mesoporous silica nanoparticles containing SPION using an alternating magnetic field. Chem. Commun. (Cambridge, U. K.) 2012, 48 (45), 5647-5649.

93. Hsu, M.-H.; Su, Y.-C. Iron-oxide embedded solid lipid nanoparticles for magnetically controlled heating and drug delivery. Biomedical microdevices 2008, 10 (6), 785-793.

94. Choubey, J.; Bajpai, A. K. Investigation on magnetically controlled delivery of doxorubicin from superparamagnetic nanocarriers of gelatin crosslinked with genipin. J. Mater. Sci.: Mater. Med. 2010, 21, 1573-1586.

95. Thomas, C. R.; Ferris, D. P.; Lee, J.-H.; Choi, E.; Cho, M. H.; Kim, E. S.; Stoddart, J. F.; Shin, J.-S.; Cheon, J.; Zink, J. I. Noninvasive Remote-Controlled Release of Drug Molecules in Vitro 
Using Magnetic Actuation of Mechanized Nanoparticles. J. Am. Chem. Soc. 2010, 132, $10623-$ 10625.

96. Elmore, W. C. Ferromagnetic Colloid for Studying Magnetic Structures. Physical Review 1938, 54, 309-310.

97. Mikhaylova, M.; Kim, D. K.; Bobrysheva, N.; Osmolowsky, M.; Semenov, V.; Tsakalakos, T.; Muhammed, M. Superparamagnetism of Magnetite Nanoparticles: Dependence on Surface Modification. Langmuir 2004, 20, 2472-2477.

98. Mikhaylova, M.; Kim, D.; Bobrysheva, N.; Osmolowsky, M.; Semenov, V.; Tsakalakos, T.; Muhammed, M. Superparamagnetism of magnetite nanoparticles: dependence on surface modification. Langmuir : the ACS journal of surfaces and colloids 2004, 20 (6), 2472-2477.

99. Biswas, S. Functionalized Nanoparticles for AMF-Induced Gene and Drug Delivery. Doctor of Philosophy in Chemistry, University of Louisville2011.

100. Yu, S.; Chow, G. M. Carboxyl group (-CO2H) functionalized ferrimagnetic iron oxide nanoparticles for potential bio-applications. Journal of Materials Chemistry 2004, 14, 2781-2786. 101. Huang, H.; Christmann, R.; Ulber, R.; Schuenemann, V. Moessbauer spectroscopy of protein-passivated iron oxide nanoparticles. Hyperfine Interact. 2012, 205, 121-124.

102. Haneda, K.; Morrish, A. H. Magnetite to maghemite transformation in ultrafine particles. J. Phys. (Paris), Colloq. 1977, 321-3.

103. Vargas, J. M.; Socolovsky, L. M.; Goya, G. F.; Knobel, M.; Zanchet, D. Structural, magnetic, and Mossbauer characterization of size-controlled iron-iron oxide nanoparticles obtained by chemical methods. IEEE Trans. Magn. 2003, 39, 2681-2683.

104. Kiwada, H.; Sato, J.; Yamada, S.; Kato, Y. Feasibility of magnetic liposomes as a targeting device for drugs. Chem. Pharm. Bull. 1986, 34 (10), 4253-8.

105. Amonette, J. E.; Templeton, J. C. Improvements to the quantitative assay of nonrefractory minerals for Fe (II) and total Fe using 1,10-phenanthroline. Clays and Clay Minerals 1998, 46 (1), 51-62.

106. Hanzlik, M.; Petersen, N.; Keller, R.; Schmidbauer, E. Electron microscopy and 57Fe Mossbauer spectra of $10 \mathrm{~nm}$ particles, intermediate in composition between Fe3O4 and $\gamma^{-}$ Fe2O3, produced by bacteria. Geophys. Res. Lett. 1996, 23 (5), 479-82.

107. Latorre, M.; Rinaldi, C. Applications of Magnetic Nanoparticles in Medicine: Magnetic Fluid Hyperthermia. Puerto Rico Health Sciences Journal 2009, 28 (3), 227-238.

108. Zhuravlev, L. T. The surface chemistry of amorphous silica. Zhuravlev model. Colloids Surf., A 2000, 173 (1-3), 1-38.

109. Galeotti, F.; Bertini, F.; Scavia, G.; Bolognesi, A. A controlled approach to iron oxide nanoparticles functionalization for magnetic polymer brushes. Journal of Colloid and Interface Science 2011, 360, 540-547.

110. Heyda, J.; Lund, M.; Oncák, M.; Slavícek, P.; Jungwirth, P. Reversal of Hofmeister ordering for pairing of $\mathrm{NH} 4(+)$ vs alkylated ammonium cations with halide anions in water. The journal of physical chemistry. B 2010, 114 (33), 10843-10852.

111. Biswas, S.; Huang, X.; Badger, W. R.; Nantz, M. H. Nucleophilic cationization reagents. Tetrahedron Letters 2010, 51 (13), 1727-1729.

112. Feng, M.; Yang, Y.; He, P.; Fang, Y. Spectroscopic studies of copper(II) and iron(II) complexes of adriamycin. Spectrochim. Acta, Part A 2000, 56A (3), 581-587.

113. Kostoryz, E. L.; Yourtee, D. M. Oxidative mutagenesis of doxorubicin-Fe(III) complex. Mutat. Res., Genet. Toxicol. Environ. Mutagen. 2001, 490 (2), 131-139.

114. Ivkov, R.; DeNardo, S. J.; Daum, W. Application of High Amplitude Alternating Magnetic Fields for Heat Induction of Nanoparticles Localized in Cancer. Clinical Cancer Research 2005, 11, 7093-7102. 
115. Heyda, J.; Lund, M.; Ončák, M.; Slavíček, P.; Jungwirth, P. Reversal of Hofmeister Ordering for Pairing of $\mathrm{NH}_{4}{ }^{+}$vs Alkylated Ammonium Cations with

Halide Anions in Water. J. Phys. Chem. B 2010, 114, 10843-10852.

116. Reeve, L. Desulfurization of coke-oven gas at Appleby-Frodingham. J. Inst. Fuel 1958, 31, 319-24.

117. Schrodt, J. T.; Yamanis, J.; Ota, K.; Sodesawa, T. Reaction of sulfides in fuel gases with the iron oxide in coal ashes. Fixed-bed experiments and simulations. Ind. Eng. Chem. Process Des. Dev. 1982, 21 (3), 382-90.

118. Miura, K.; Mae, K.; Inoue, T.; Yoshimi, T.; Nakagawa, H.; Hashimoto, K. Simultaneous removal of carbonyl sulfide and hydrogen sulfide from coke oven gas at low temperature by iron oxide. Ind. Eng. Chem. Res. 1992, 31 (1), 415-19.

119. Lin Wang, F. Z., Jianmin Chen. Carbonyl Sulfide Derived from Catalytic Oxidation of Carbon Disulfide over Atmospheric Particles. Environmental Science \& Technology 2001, 35, 2543-2547.

120. Chen, H.; Kong, L.; Chen, J.; Zhang, R.; Wang, L. Heterogeneous Uptake of Carbonyl Sulfide on Hematite and Hematite-NaCl Mixtures. Environ. Sci. Technol. 2007, 41 (18), 64846490 .

121. Kwan, T.; Fujita, Y. Chemisorption of $\mathrm{CO}_{2}$ over oxide catalysts of spinel type. Journal of the Research Institute for Catalysis Hokkaido University 1953, 2 (2), 110-116.

122. Parkinson, G.; Novotný, Z.; Jacobson, P.; Schmid, M.; Diebold, U. Room temperature water splitting at the surface of magnetite. Journal of the American Chemical Society 2011, 133 (32), 12650-12655.

123. Chang, Y.-S.; Savitha, S.; Sadhasivam, S.; Hsu, C.-K.; Lin, F.-H. Fabrication, characterization, and application of greigite nanoparticles for cancer hyperthermia. Journal of Colloid and Interface Science 2011, 363 (1), 314-319.

124. McCarthy, S. A.; Davies, G.-L.; Gun'ko, Y. K. Preparation of multifunctional nanoparticles and their assemblies. Nat. Protoc. 2012, 7, 1677-1693.

125. Li, C.; Ma, C.; Wang, F.; Xi, Z.; Wang, Z.; Deng, Y.; He, N. Preparation and biomedical applications of core-shell silica/magnetic nanoparticle composites. J. Nanosci. Nanotechnol. 2012, 12 (4), 2964-2972.

126. Malvindi, M. A.; De Matteis, V.; Galeone, A.; Brunetti, V.; Anyfantis, G. C.; Athanassiou, A.; Cingolani, R.; Pompa, P. P. Toxicity assessment of silica coated iron oxide nanoparticles and biocompatibility improvement by surface engineering. PLoS One 2014, 9 (1), e85835/1e85835/11, 11 pp.

127. Pinho, S. L. C.; Pereira, G. A.; Voisin, P.; Kassem, J.; Bouchaud, V.; Etienne, L.; Peters, J. A.; Carlos, L.; Mornet, S.; Geraldes, C. F. G. C.; Rocha, J.; Delville, M.-H. Fine Tuning of the Relaxometry of $\gamma$-Fe2O3@SiO2 Nanoparticles by Tweaking the Silica Coating Thickness. ACS Nano 2010, 4, 5339-5349.

128. Villanueva, A.; Cañete, M.; Roca, A.; Calero, M.; Veintemillas-Verdaguer, S.; Serna, C.; Morales, M.; Miranda, R. The influence of surface functionalization on the enhanced internalization of magnetic nanoparticles in cancer cells. Nanotechnology 2009, 20 (11), 115103. 129. Zhang, Y.; Yang, M.; Portney, N.; Cui, D.; Budak, G.; Ozbay, E.; Ozkan, M.; Ozkan, C. Zeta potential: a surface electrical characteristic to probe the interaction of nanoparticles with normal and cancer human breast epithelial cells. Biomedical microdevices 2008, 10 (2), 321-328. 130. De Cuyper, M.; Joniau, M. Magnetoliposomes. European Biophysics Journal 1987, 15, 311-319. 
131. Soenen, S.; Hodenius, M.; De Cuyper, M. Magnetoliposomes: versatile innovative nanocolloids for use in biotechnology and biomedicine. Nanomedicine (London, England) 2009, 4 (2), 177-191.

132. de Cuyper, M.; Soenen, S. J. H. Cationic magnetoliposomes. Methods Mol. Biol. (Totowa, NJ, U. S.) 2010, 605 (Liposomes, Volume 1), 97-111.

133. Soenen, S. J.; De Meyer, S. F.; Dresselaers, T.; Velde, G. V.; Pareyn, I. M.; Braeckmans, K.; De Cuyper, M.; Himmelreich, U.; Vanhoorelbeke, K. I. MRI assessment of blood outgrowth endothelial cell homing using cationic magnetoliposomes. Biomaterials 2011, 32 (17), 4140 4150 .

134. Soenen, S. J. H.; Baert, J.; De Cuyper, M. Optimal conditions for labelling of 3T3 fibroblasts with magnetoliposomes without affecting cellular viability. ChemBioChem 2007, 8 (17), 2067-2077.

135. Soenen, S. J. H.; Brisson, A. R.; De Cuyper, M. Addressing the problem of cationic lipidmediated toxicity: The magnetoliposome model. Biomaterials 2009, 30 (22), 3691-3701.

136. Soenen, S. J. H.; Brisson, A. R.; Jonckheere, E.; Nuytten, N.; Tan, S.; Himmelreich, U.; De Cuyper, $\mathrm{M}$. The labeling of cationic iron oxide nanoparticle-resistant hepatocellular carcinoma cells using targeted magnetoliposomes. Biomaterials 2011, 32 (6), 1748-1758.

137. Soenen, S. J. H.; De Cuyper, M. How to assess cytotoxicity of (iron oxide-based) nanoparticles. A technical note using cationic magnetoliposomes. Contrast Media Mol. Imaging 2011, 6 (3), 153-164.

138. Soenen, S. J. H.; Illyes, E.; Vercauteren, D.; Braeckmans, K.; Majer, Z.; De Smedt, S. C.; De Cuyper, $\mathrm{M}$. The role of nanoparticle concentration-dependent induction of cellular stress in the internalization of non-toxic cationic magnetoliposomes. Biomaterials 2009, 30 (36), 6803-6813. 139. Soenen, S. J. H.; Nuytten, N.; Himmelreich, U.; De Cuyper, M. The Possible Side-Effects Of Iron Oxide Nanoparticles On Cell Functionality. AIP Conf. Proc. 2010, 1275 (Bonsai Project Symposium, 2010), 106-111.

140. Soenen, S. J. H.; Vercauteren, D.; Braeckmans, K.; Noppe, W.; De Smedt, S.; De Cuyper, M. Stable long-term intracellular labeling with fluorescently tagged cationic magnetoliposomes. ChemBioChem 2009, 10 (2), 257-267.

141. Biswas, S.; Knipp, R. J.; Gordon, L. E.; Nandula, S. R.; Gorr, S.-U.; Clark, G. J.; Nantz, M. H. Hydrophobic Oxime Ethers: A Versatile Class of pDNA and siRNA Transfection Lipids.

ChemMedChem 2011, 6, 2063-2069.

142. Junquera, E.; Aicart, E. Cationic Lipids as Transfecting Agents of DNA in Gene Therapy.

Curr. Top. Med. Chem. (Sharjah, United Arab Emirates) 2014, 14 (5), 649-663.

143. Pan, X.; Guan, J.; Yoo, J.-W.; Epstein, A. J.; Lee, L. J.; Lee, R. J. Cationic lipid-coated magnetic nanoparticles associated with transferrin for gene delivery. Int. J. Pharm. 2008, 358 (12), 263-270.

144. Jiang, S.; Eltoukhy, A.; Love, K.; Langer, R.; Anderson, D. Lipidoid-coated iron oxide nanoparticles for efficient DNA and siRNA delivery. Nano letters 2013, 13 (3), 1059-1064.

145. Biswas, S.; Gordon, L. E.; Clark, G. J.; Nantz, M. H. Click assembly of magnetic nanovectors for gene delivery. Biomaterials 2011, 32, 2683-2688.

146. Zhi, D.; Zhang, S.; Cui, S.; Zhao, Y.; Wang, Y.; Zhao, D. The Headgroup Evolution of Cationic Lipids for Gene Delivery. Bioconjugate Chem. 2013, 24 (4), 487-519.

147. Hanson, R. M. The synthetic methodology of nonracemic glycidol and related 2,3-epoxy alcohols. Chem. Rev. 1991, 91 (4), 437-76.

148. Azizi, N.; Saidi, M. Highly chemoselective addition of amines to epoxides in water.

Organic letters 2005, 7 (17), 3649-3651. 
149. Heydari, A. Organic synthesis in an unconventional solvent, 5.0 M lithium perchlorate/diethyl ether. Tetrahedron 2002.

150. Kuwabe, S.; Torraca, K.; Buchwald, S. Palladium-catalyzed intramolecular C-O bond formation. Journal of the American Chemical Society 2001, 123 (49), 12202-12206.

151. Effenberger, K.; Breyer, S.; Ocker, M.; Schobert, R. New doxorubicin N-acyl hydrazones with improved efficacy and cell line specificity show modes of action different from the parent drug. Int J Clin Pharmacol Ther 2010, 48 (7), 485-6.

152. Effenberger, K.; Breyer, S.; Schobert, R. Modulation of doxorubicin activity in cancer cells by conjugation with fatty acyl and terpenyl hydrazones. European journal of medicinal chemistry 2010, 45 (5), 1947-1954.

153. Frézard, F.; Garnier-Suillerot, A. Permeability of lipid bilayer to anthracycline derivatives. Role of the bilayer composition and of the temperature. Biochimica et Biophysica Acta (BBA)Lipids ... 1998.

154. Krüger, M.; Beyer, U.; Schumacher, P.; Unger, C.; Zahn, H.; Kratz, F. Synthesis and stability of four maleimide derivatives of the anticancer drug doxorubicin for the preparation of chemoimmunoconjugates. Chem. Pharm. Bull. 1997, 45, 399-401.

155. Graeser, R.; Esser, N.; Unger, H.; Fichtner, I.; Zhu, A.; Unger, C.; Kratz, F. INNO-206, the (6-maleimidocaproyl hydrazone derivative of doxorubicin), shows superior antitumor efficacy compared to doxorubicin in different tumor xenograft models and in an orthotopic pancreas carcinoma model. Investigational new drugs 2010, 28 (1), 14-19.

156. Dirksen, A.; Dawson, P. Rapid oxime and hydrazone ligations with aromatic aldehydes for biomolecular labeling. Bioconjugate chemistry 2008, 19 (12), 2543-2548.

157. Petralito, S.; Spera, R.; Memoli, A.; D'Inzeo, G.; Liberti, M.; Apollonio, F. Preparation and characterization of lipid vesicles entrapping iron oxide nanoparticles. Asia-Pac. J. Chem. Eng.

2012, 7 (S3), S335-S341.

158. Laouini, A.; Jaafar-Maalej, C.; Limayem-Blouza, I.; Sfar, S.; Charcosset, C.; Fessi, H. Preparation, Characterization and Applications of Liposomes: State of the Art. Journal of Colloid Science and Biotechnology 2012, 1, 147-168.

159. Niculescu-Duvaz, D.; Heyes, J.; Springer, C. J. Structure-activity relationship in cationic lipid mediated gene transfection. Curr. Med. Chem. 2003, 10 (14), 1233-1261.

160. Nantz, M. H.; Li, L.; Zhu, J.; Aho-Sharon, K. L.; Lim, D.; Erickson, K. L. Inductive ElectronWithdrawal From Ammonium Ion Headgroups of Cationic Lipids and the Influence on DNA Transfection. Biochimica et Biophysica Acta 1998, 1394, 219-223.

161. Le Corre, S. S.; Berchel, M.; Le Gall, T.; Haelters, J.-P.; Lehn, P.; Montier, T.; Jaffres, P.-A. Cationic Trialkylphosphates: Synthesis and Transfection Efficacies Compared to Phosphoramidate Analogues. Eur. J. Org. Chem. 2014, Ahead of Print.

162. Nantz, M. H.; Dicus, C. W.; Hilliard, B.; Yellayi, S.; Zou, S.; Hecker, J. G. The benefit of hydrophobic domain asymmetry on the efficacy of transfection as measured by in vivo imaging. Mol. Pharmaceutics 2010, 7 (3), 786-794.

163. Heyes, J. A.; Niculescu-Duvaz, D.; Cooper, R. G.; Springer, C. J. Synthesis of Novel Cationic Lipids: Effect of Structural Modification on the Efficiency of Gene Transfer. J. Med. Chem. 2002, 45 (1), 99-114.

164. Santel, A.; Aleku, M.; Keil, O.; Endruschat, J.; Esche, V.; Fisch, G.; Dames, S.; Loeffler, K.; Fechtner, M.; Arnold, W.; Giese, K.; Klippel, A.; Kaufmann, J. A novel siRNA-lipoplex technology for RNA interference in the mouse vascular endothelium. Gene Ther. 2006, 13 (16), 1222-1234. 165. Bennett, M. J.; Aberle, A. M.; Balasubramaniam, R. P.; Malone, J. G.; Malone, R. W.; Nantz, M. H. Cationic lipid-mediated gene delivery to murine lung: correlation of lipid hydration with in vivo transfection activity. J. Med. Chem. 1997, 40 (25), 4069-4078. 
166. Gottlieb, H. E.; Kotlyar, V.; Nudelman, A. NMR Chemical Shifts of Common Laboratory Solvents as Trace Impurities. J. Org. Chem. 1997, 62, 7512-7515.

167. Buruiana, E. C.; Buruiana, T.; Melinte, V.; Negulescu, I. Synthesis, Structure and Mesogenic Properties of Some Alkylammonium Derivatives. Revue Roumaine de Chimie 2005, 50 (6), 465-470.

168. Tsubone, K.; Uchida, N.; Niwase, H.; Honda, K. Syntheses of Sodium 2-(N-alkyl-Nmethylamino)ethanephosphates and Their Physicochemical Properties. Journal of the American Oil Chemists' Society 1989, 66 (6), 829-833.

169. Fan, T. W.-M.; Lane, A. N.; Higashi, R. M.; Yan, J. Stable isotope resolved metabolomics of lung cancer in a SCID mouse model. Metabolomics 2010.

170. Kim, B.; Moon, S.; Hwang, B. Structure elucidation and antifungal activity of an anthracycline antibiotic, daunomycin, isolated from Actinomadura roseola. Journal of Agricultural and Food Chemistry 2000, 48 (5), 1875-1881.

171. Sommer, H.; Lipp, H.; Jackson, L. Alkylation of amines. General exhaustive alkylation method for the synthesis of quaternary ammonium compounds. The Journal of Organic ... 1971. 172. Mancuso, A. J.; Huang, S.-L.; Swern, D. Oxidation of long-chain and related alcohols to carbonyls by dimethyl sulfoxide "activated" by oxalyl chloride. J. Org. Chem. 1978, 43, 2480-2. 173. Kuwabe, S.-i.; Torraca, K. E.; Buchwald, S. L. Palladium-Catalyzed Intramolecular C-O Bond Formation. J. Am. Chem. Soc. 2001, 123, 12202-12206. 


\title{
APPENDIX
}

\author{
A.1. NMR spectra
}

A.2. Zeta potential measurements

A.3. Excitation-emission spectra

A.4. Dynamic light scattering measurements

A.5. Fluorescence and bright-field microscope images

A.6. Copyright permissions 


\section{A.1. NMR SPECTRA}




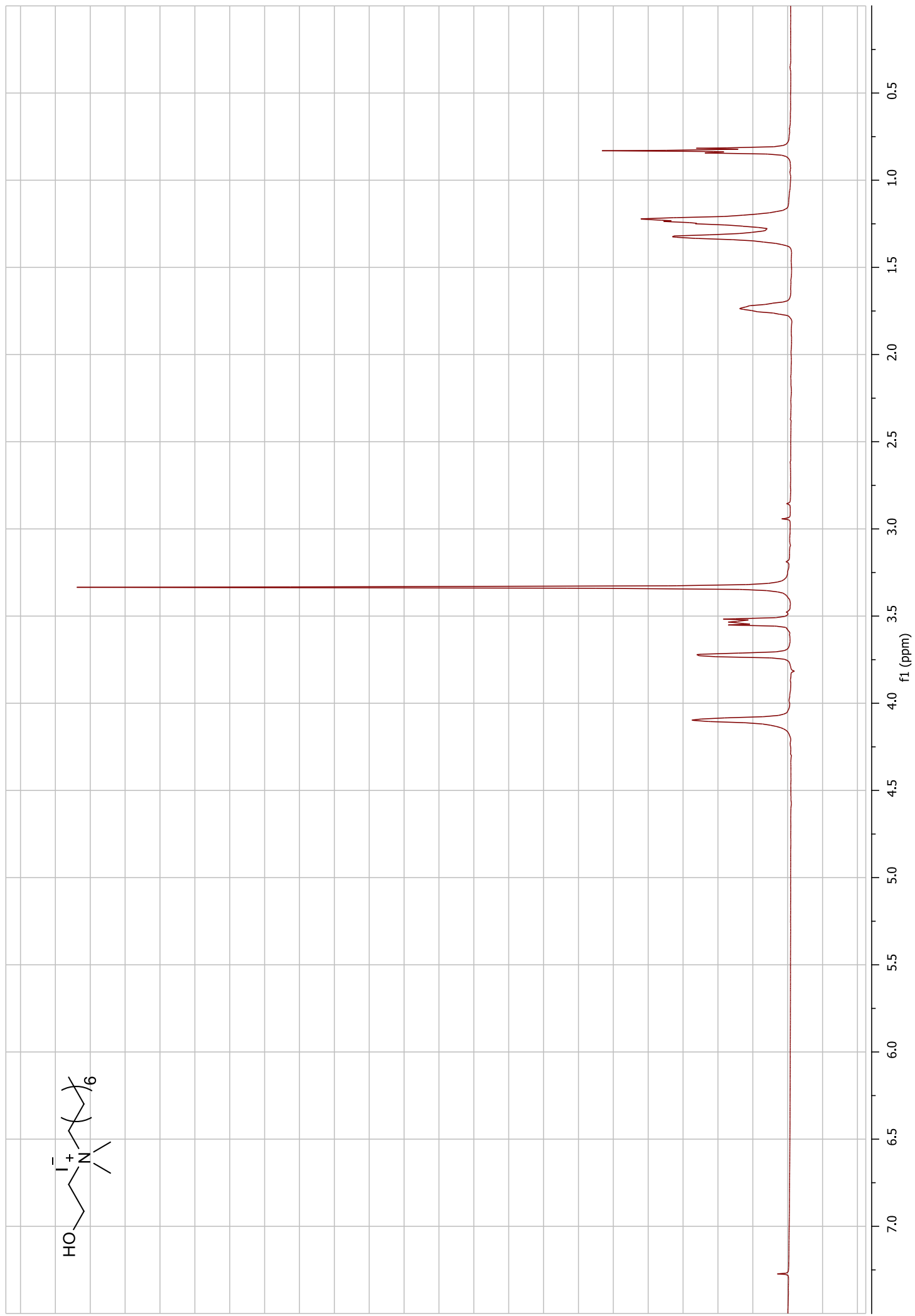

${ }^{1} \mathrm{H}$ NMR of $2.1500 \mathrm{MHz} \mathrm{CDCl}_{3}$ 


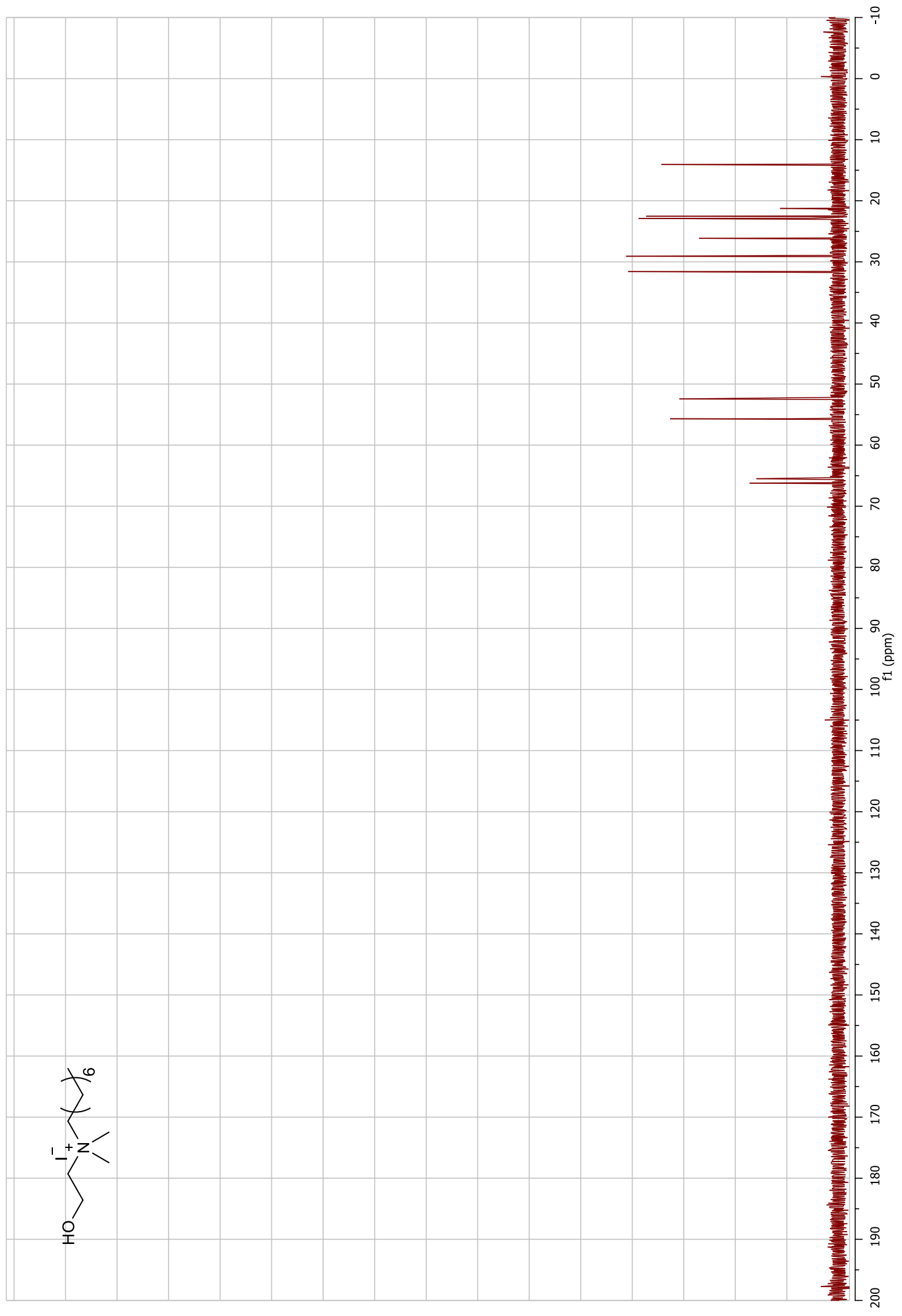

${ }^{13} \mathrm{C}$ NMR of $2.1125 \mathrm{MHz}$ acetone- $\mathrm{d}_{6}$ 
(udd) If

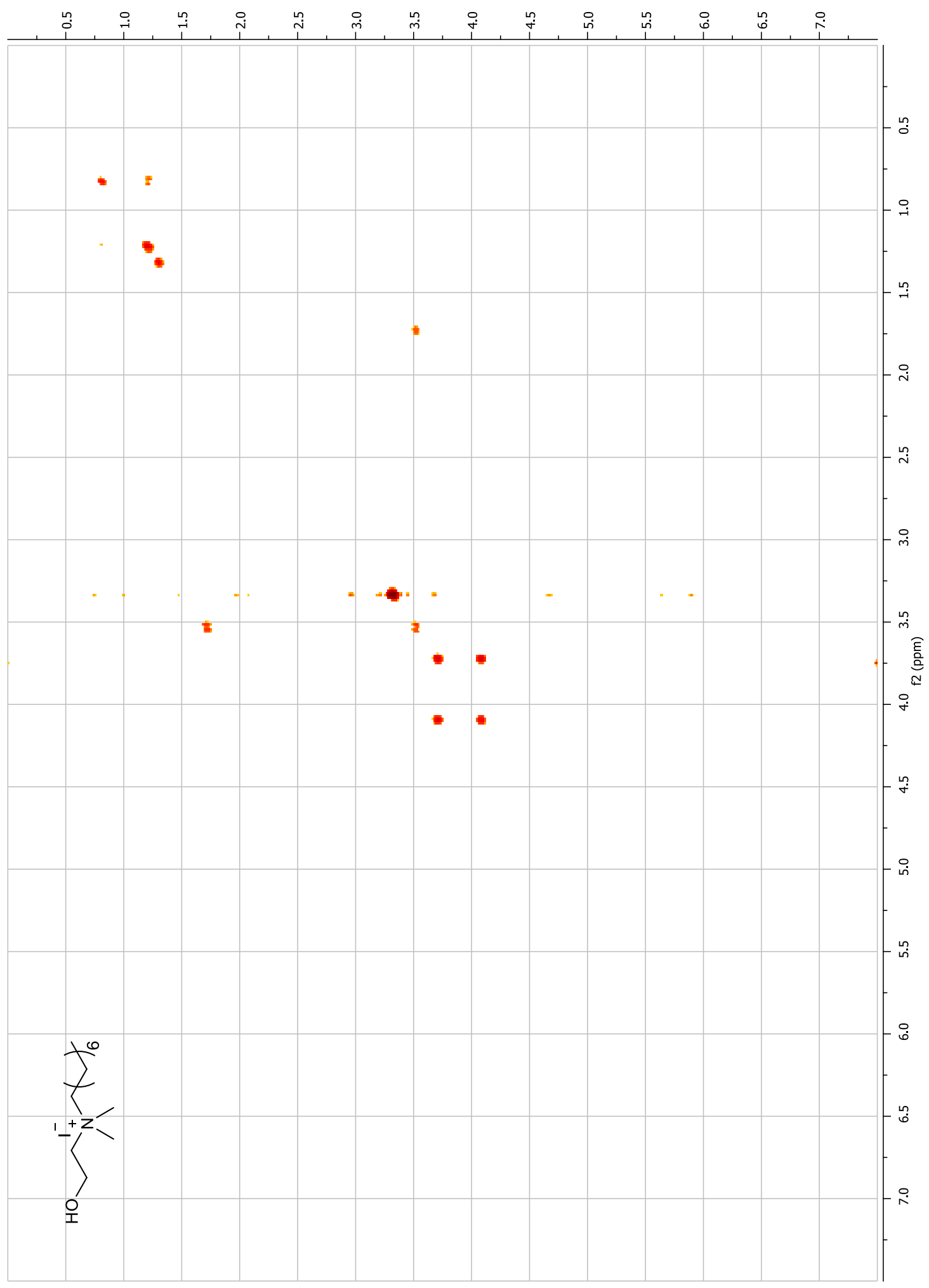

${ }^{1} \mathrm{H}-{ }^{1} \mathrm{H}$ COSI of $2.1500 \mathrm{MHz} \mathrm{CDCl}_{3}$ 


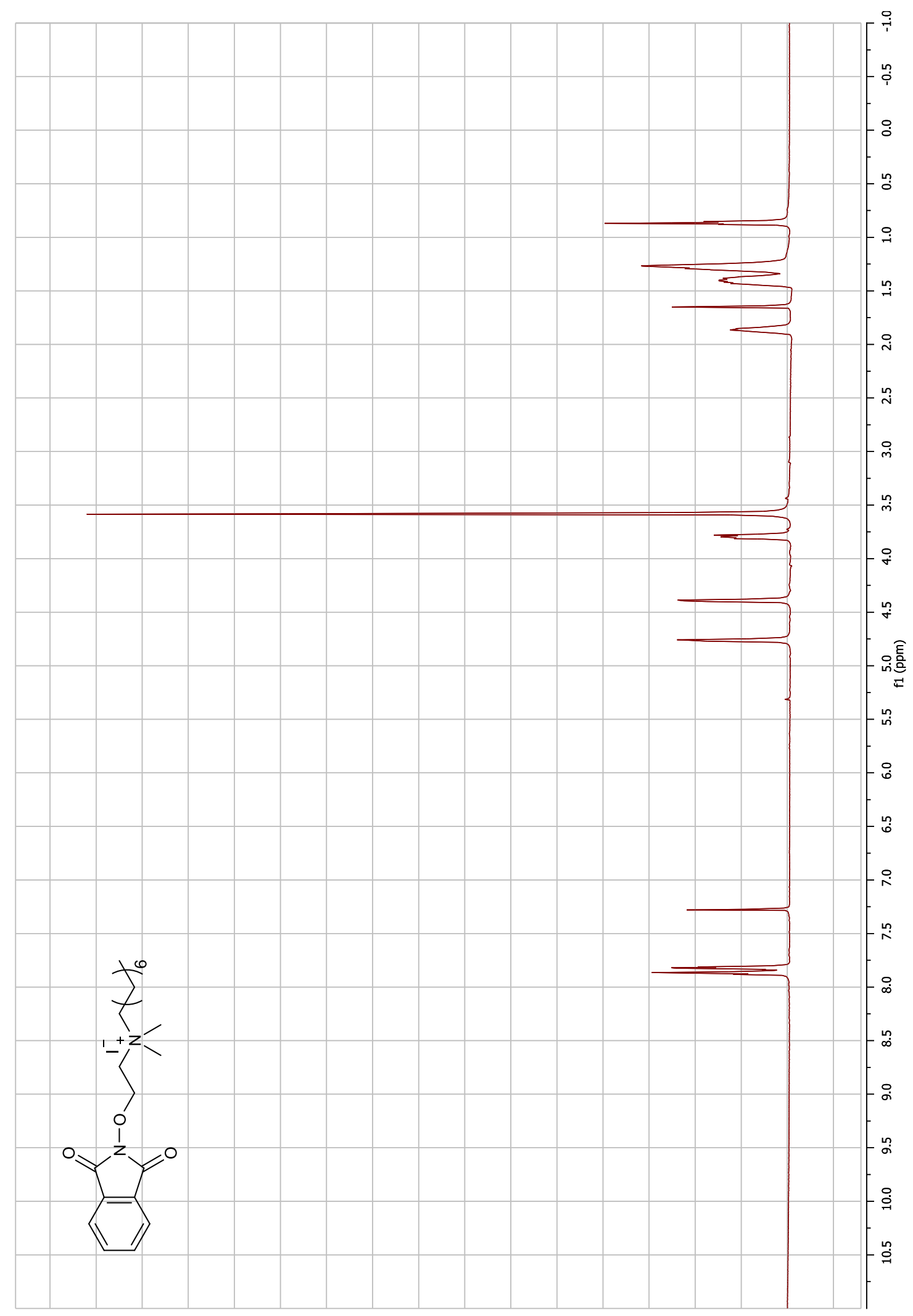

${ }^{1} \mathrm{H}$ NMR of $2.2500 \mathrm{MHz}^{\mathrm{CDCl}}{ }_{3}$ 


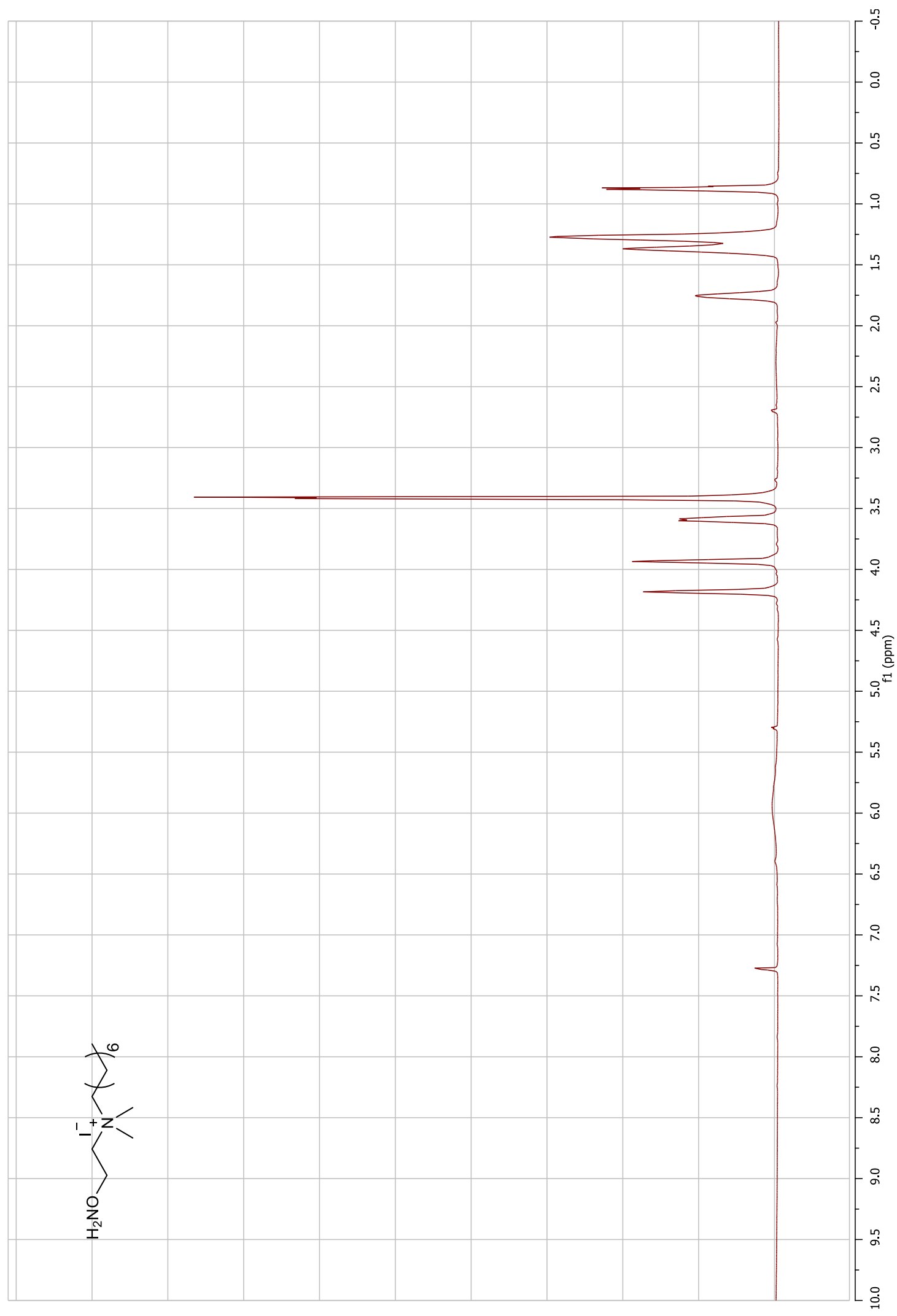

${ }^{1} \mathrm{H}$ NMR of QOA $500 \mathrm{MHz} C D C l_{3}$ 


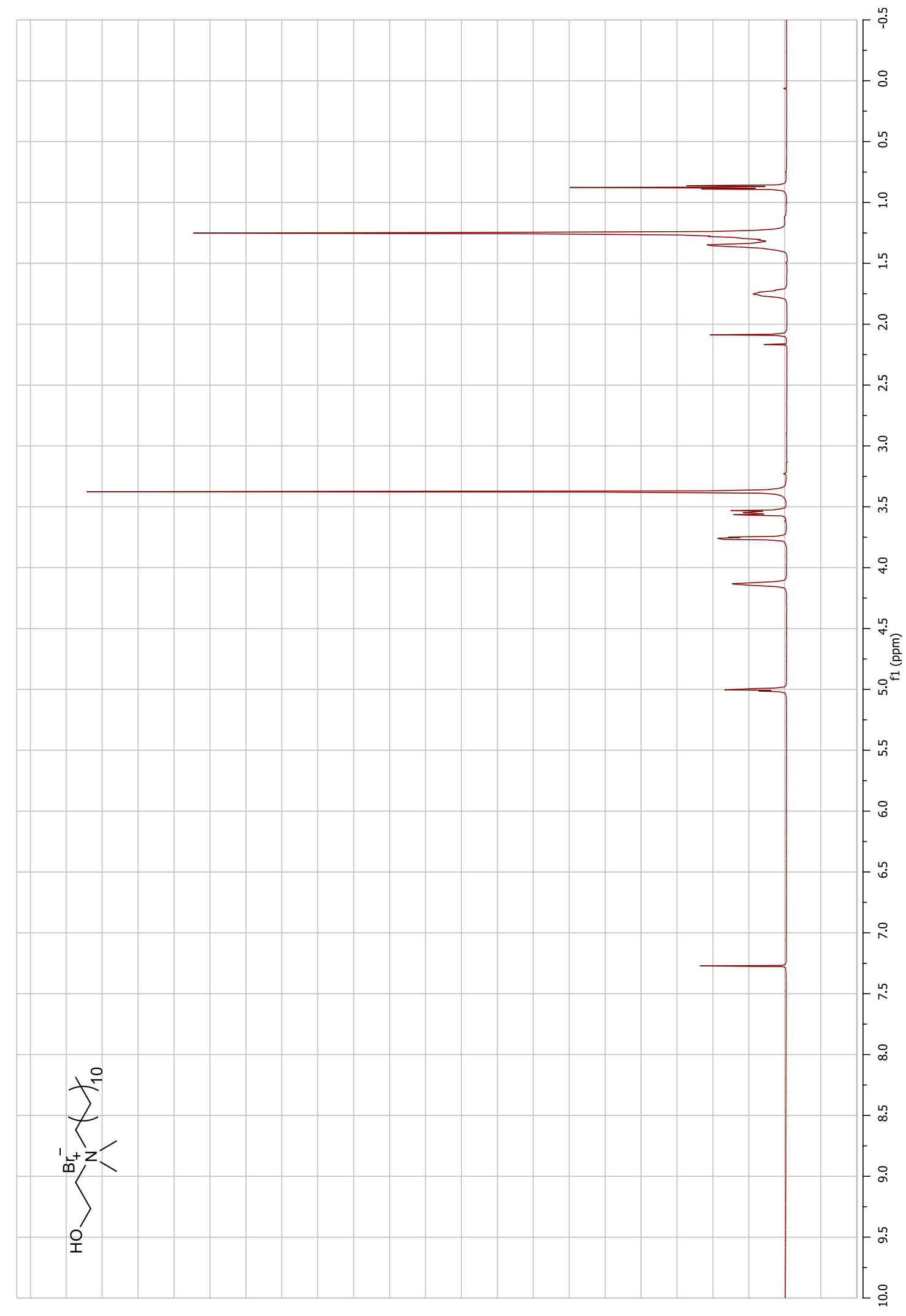

${ }^{1} \mathrm{H}$ NMR of $2.3500 \mathrm{MHz} \mathrm{CDCl}_{3}$ 


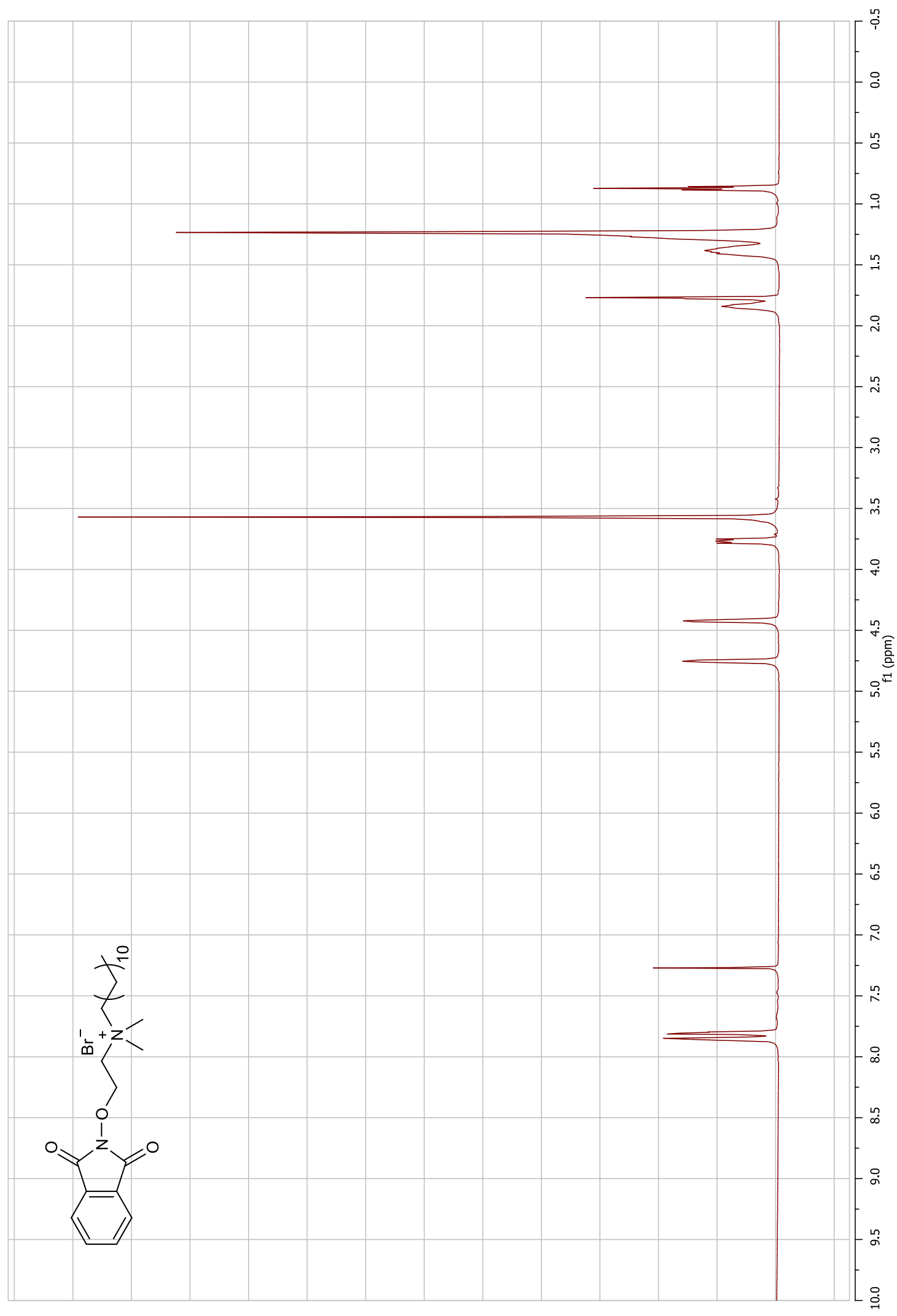

${ }^{1} \mathrm{H}$ NMR of $2.4500 \mathrm{MHz} \mathrm{CDCl}_{3}$ 


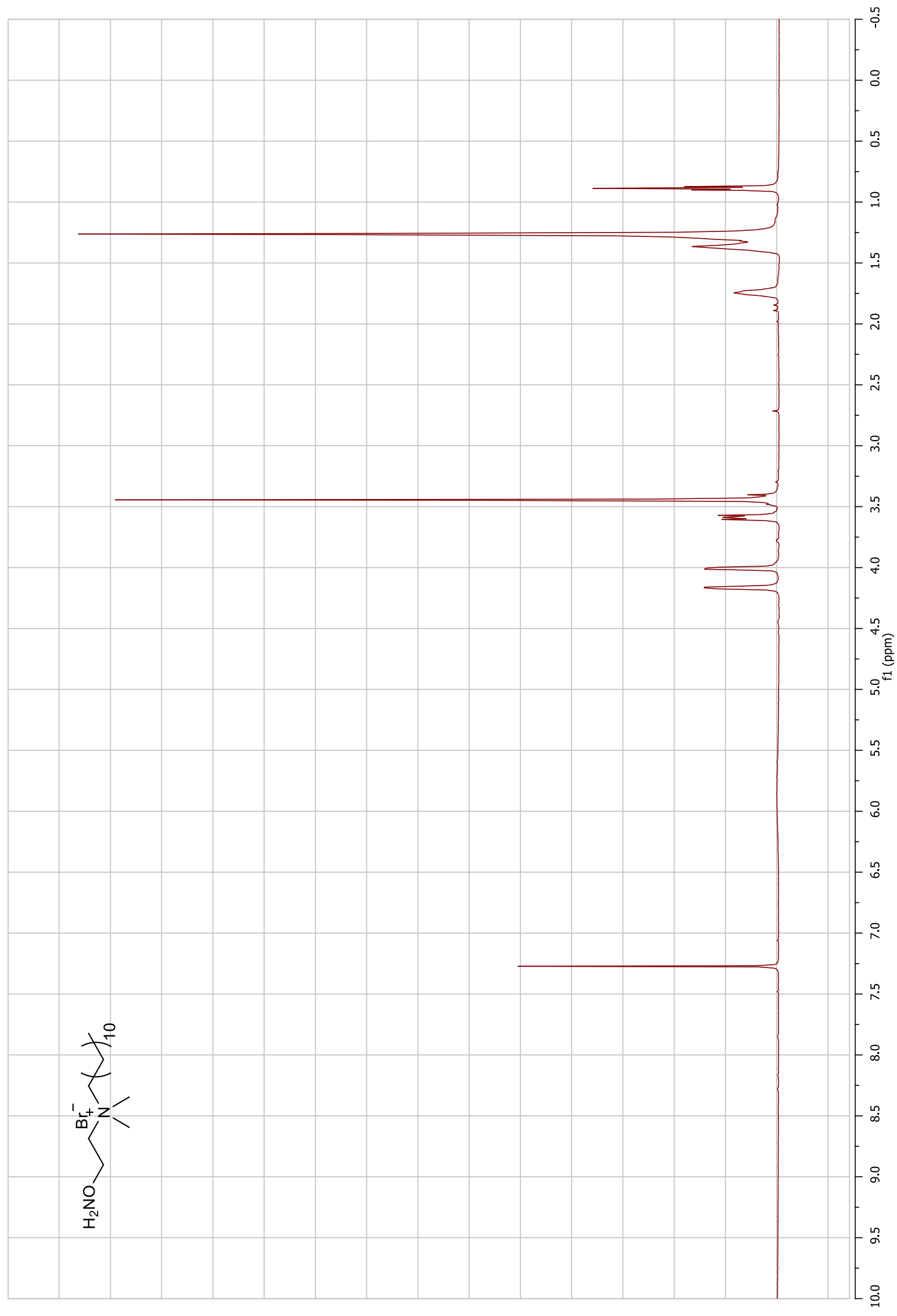

${ }^{1} \mathrm{H} N M R$ of QDA. $\mathrm{Br} 500 \mathrm{MHz} \mathrm{CDCl}_{3}$ 


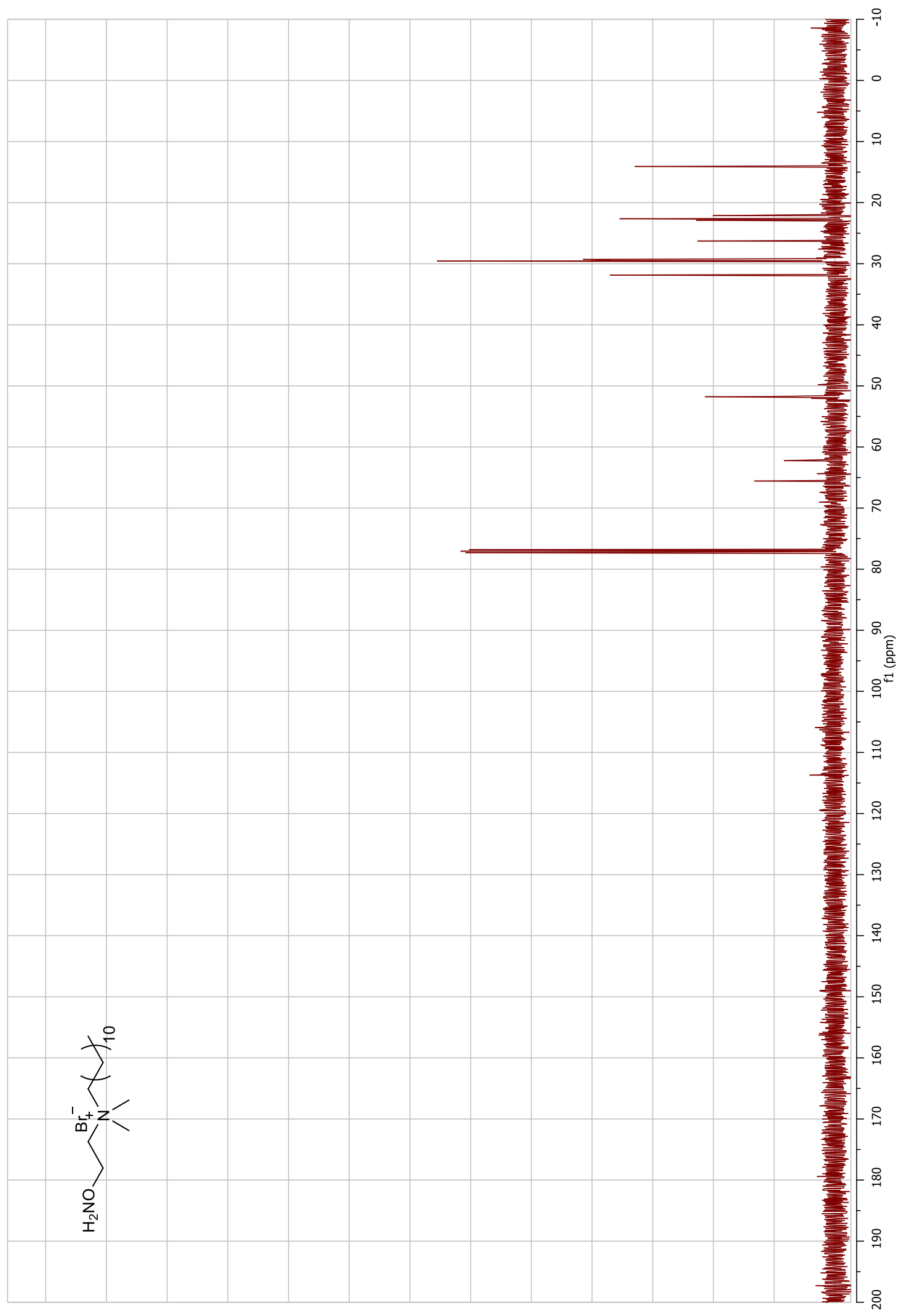

${ }^{13} \mathrm{C}$ NMR of QDA. $\mathrm{Br} 125 \mathrm{MHz} \mathrm{CDCl}_{3}$ 


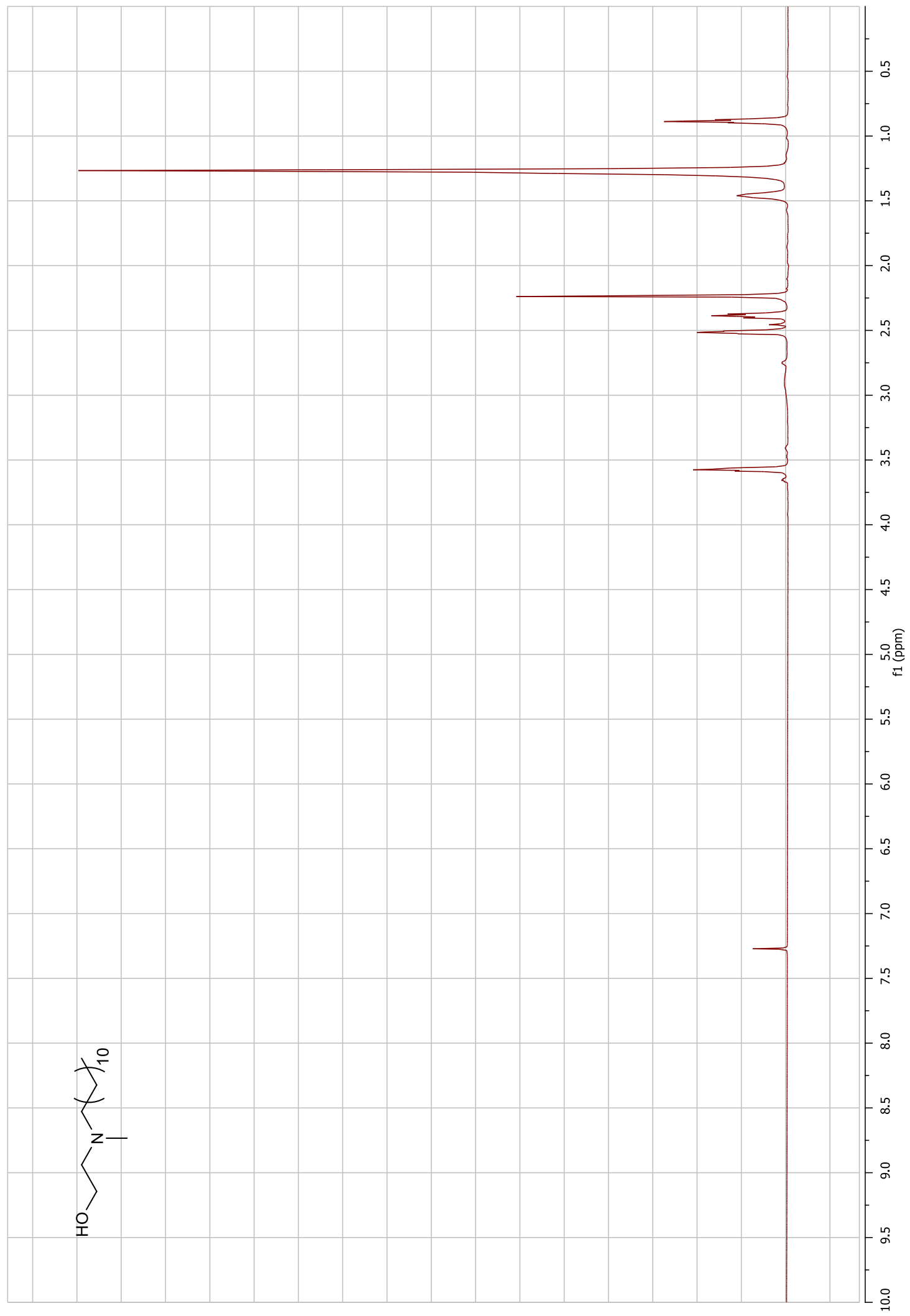

${ }^{1} \mathrm{H}$ NMR of $2.6500 \mathrm{MHz}^{\mathrm{CDCl}}{ }_{3}$ 


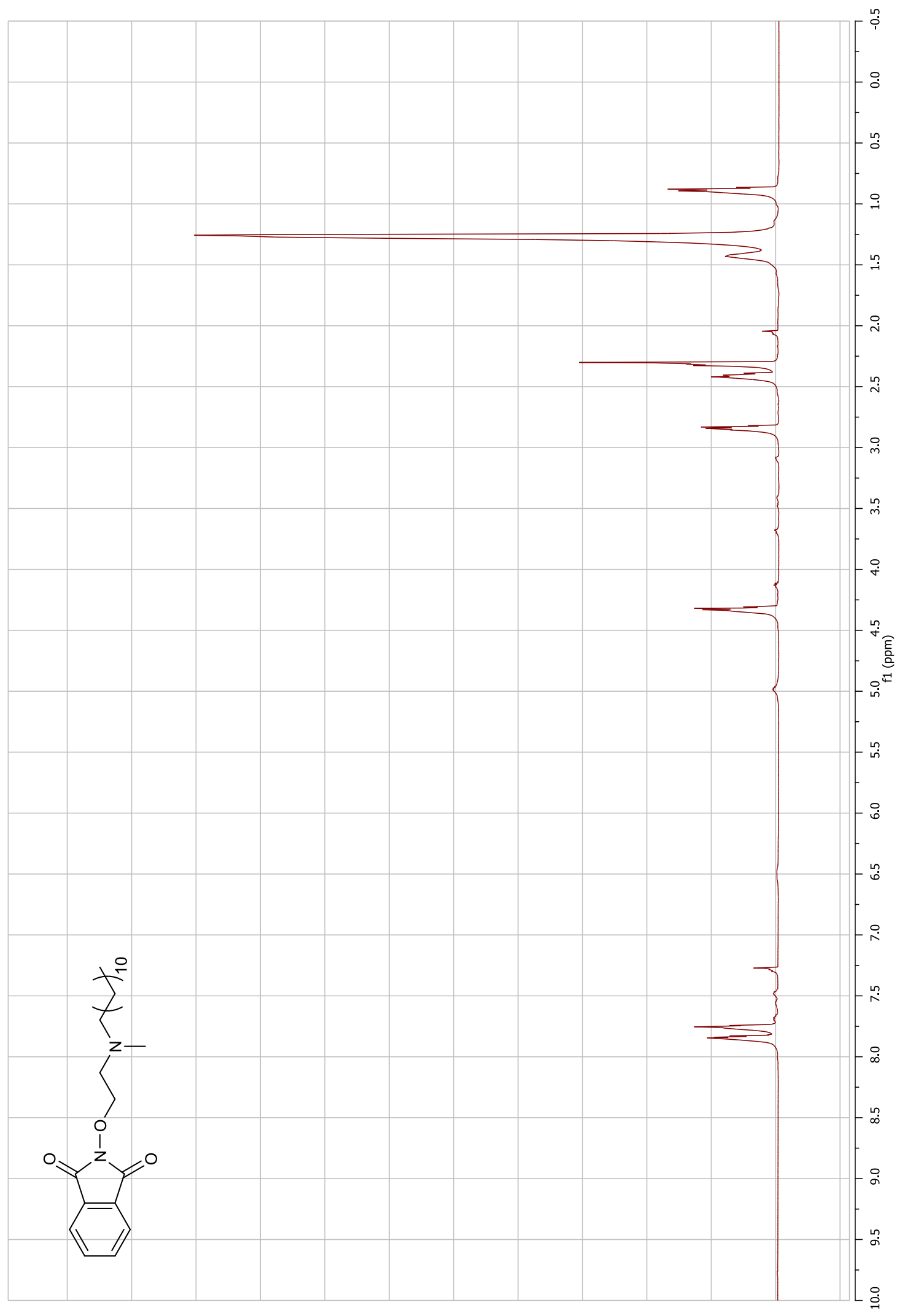

${ }^{1} \mathrm{H}$ NMR of $2.7400 \mathrm{MHz}^{\mathrm{CDCl}} 3$ 


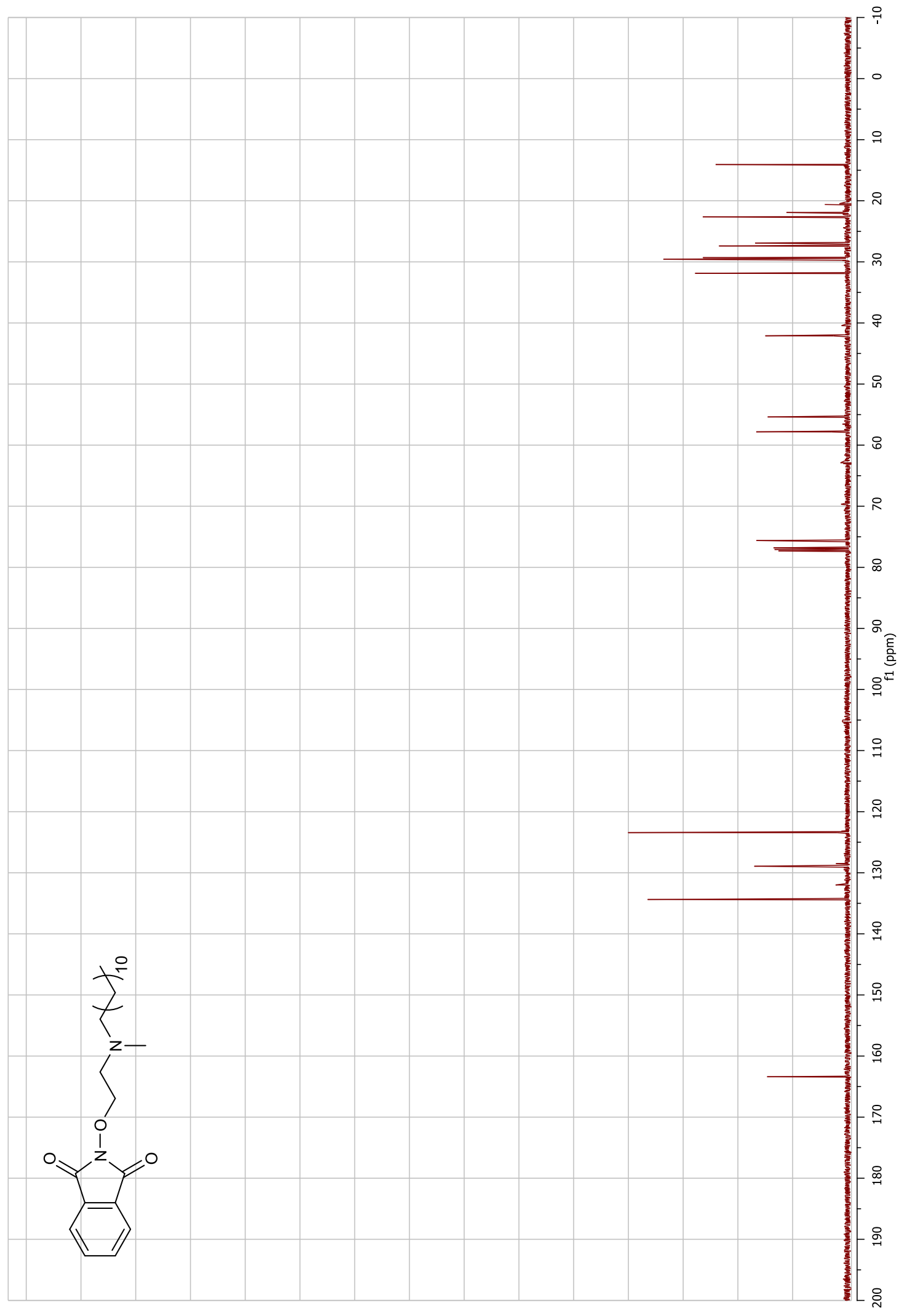

${ }^{13} \mathrm{C}$ NMR of $2.7125 \mathrm{MHz}^{\mathrm{CDCl}} 3$ 


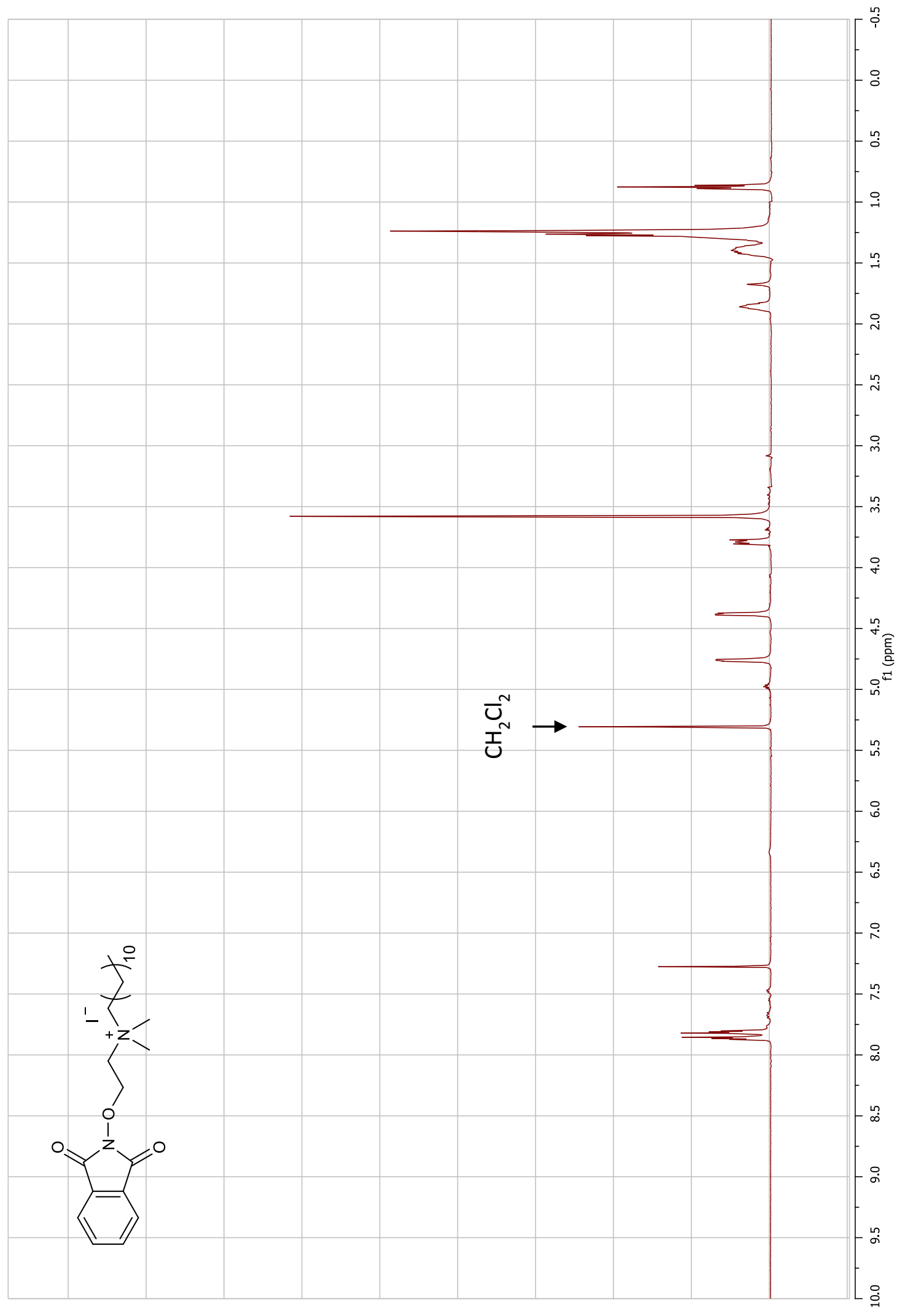

${ }^{1} \mathrm{H}$ NMR of $2.8500 \mathrm{MHz} \mathrm{CDCl}_{3}$ 


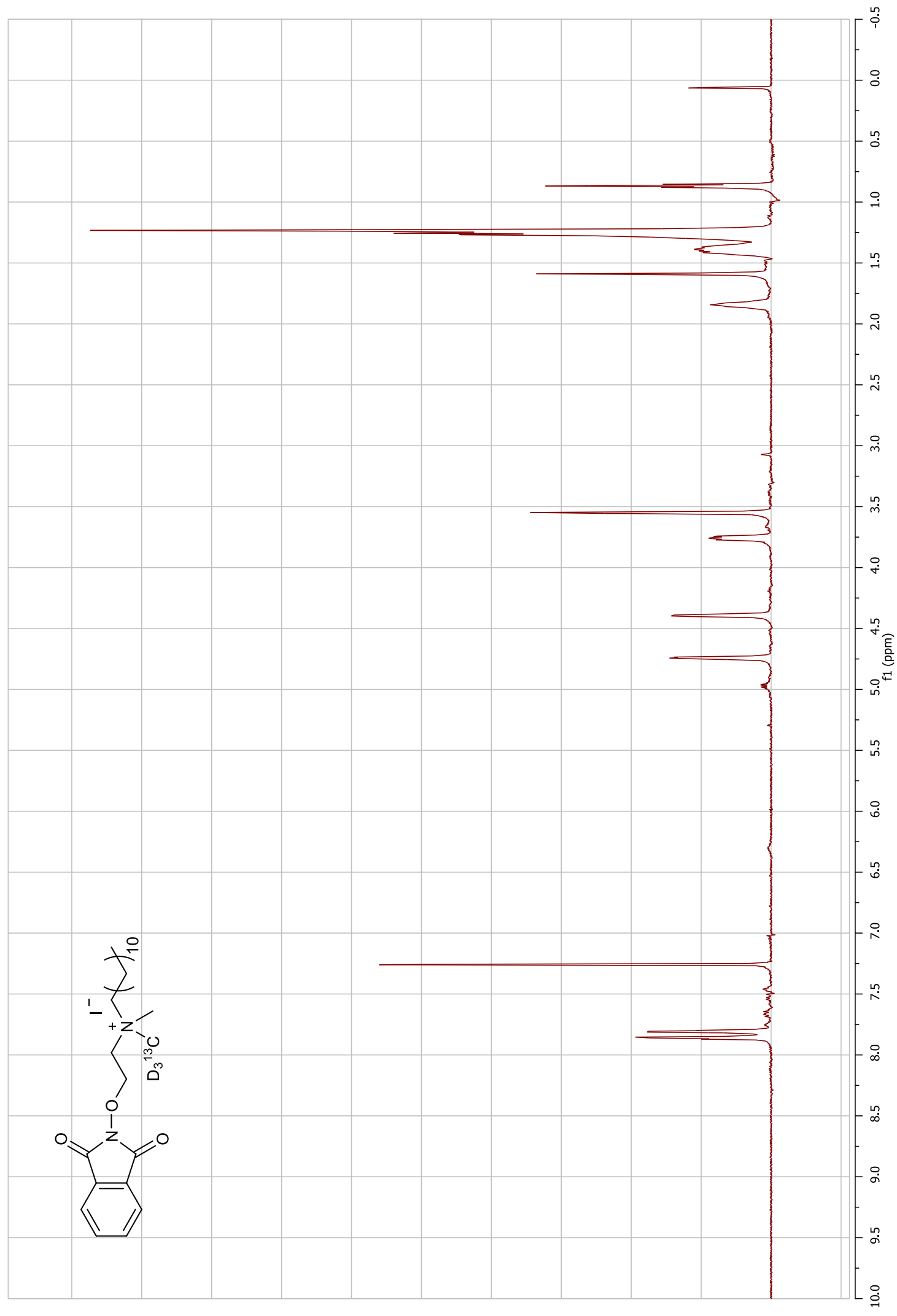

${ }^{1} \mathrm{H}$ NMR of $2.9500 \mathrm{MHz} \mathrm{CDCl}_{3}$ 


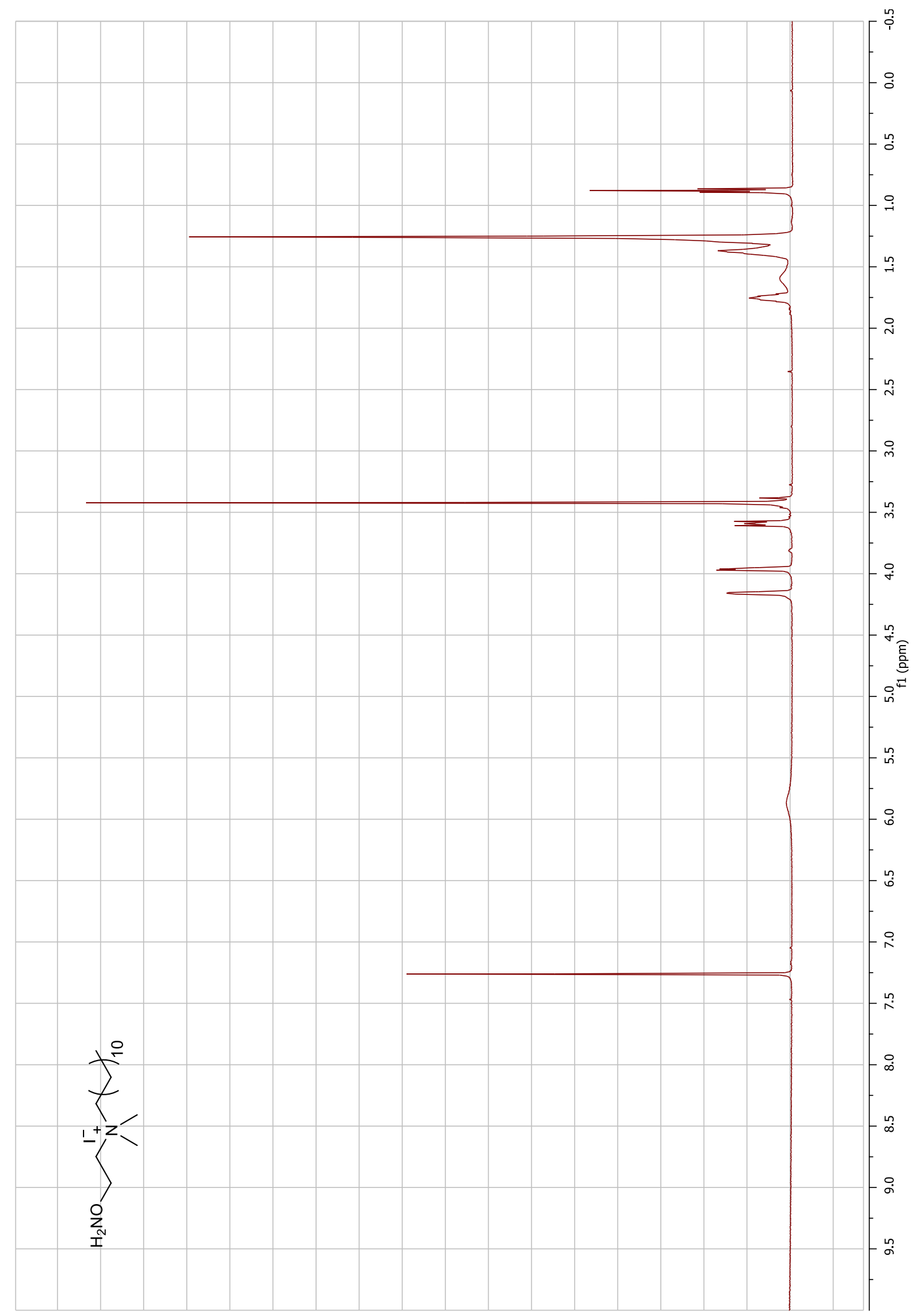

${ }^{1} \mathrm{H}$ NMR of QDA $500 \mathrm{MHz}^{\mathrm{CDCl}_{3}}$ 


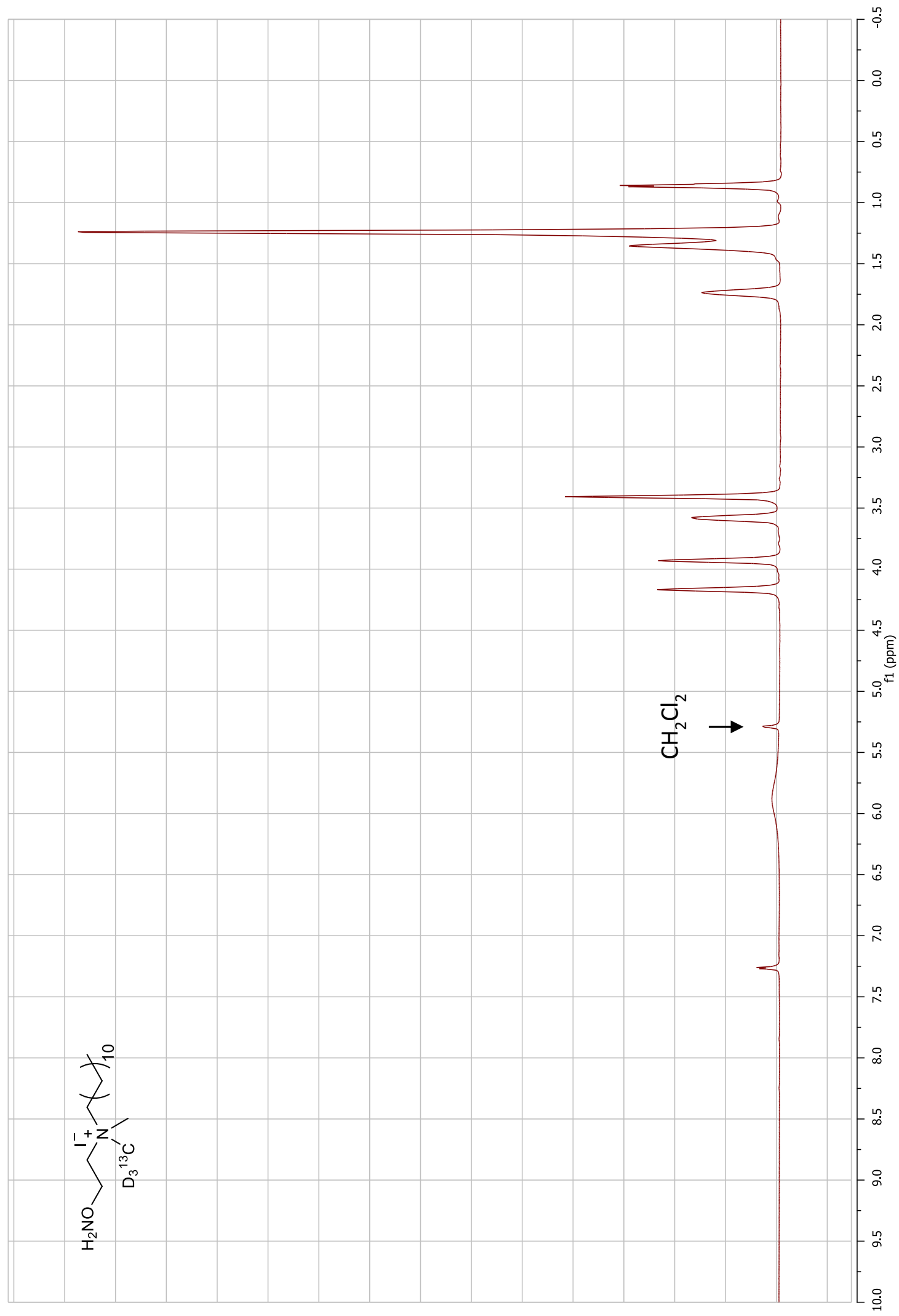

${ }^{1} \mathrm{H}$ NMR of *QDA $500 \mathrm{MHz} \mathrm{CDCl}_{3}$ 


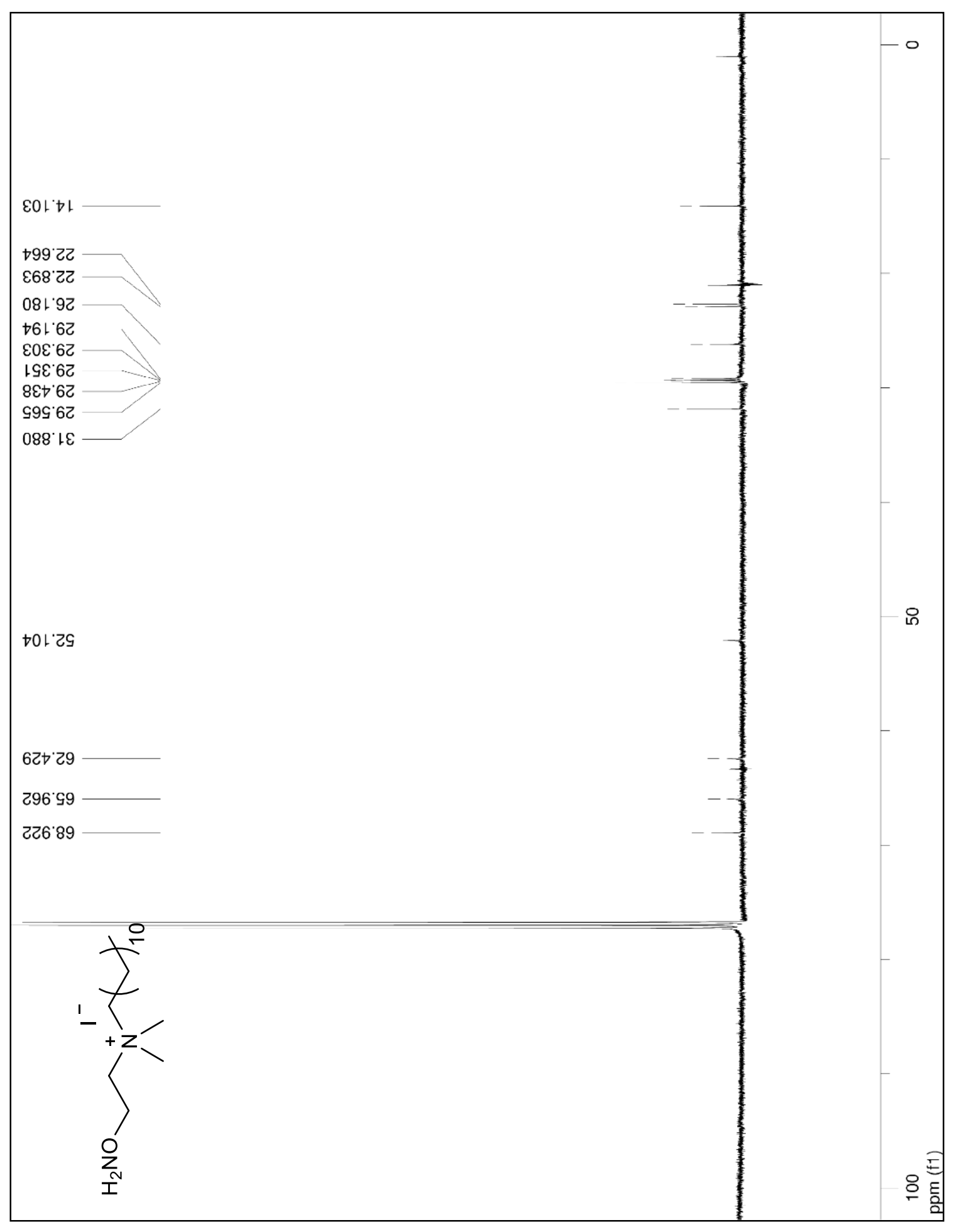

${ }^{13} \mathrm{C}$ NMR of QDA $500 \mathrm{MHz}$ in $\mathrm{CDCl}_{3}$ 


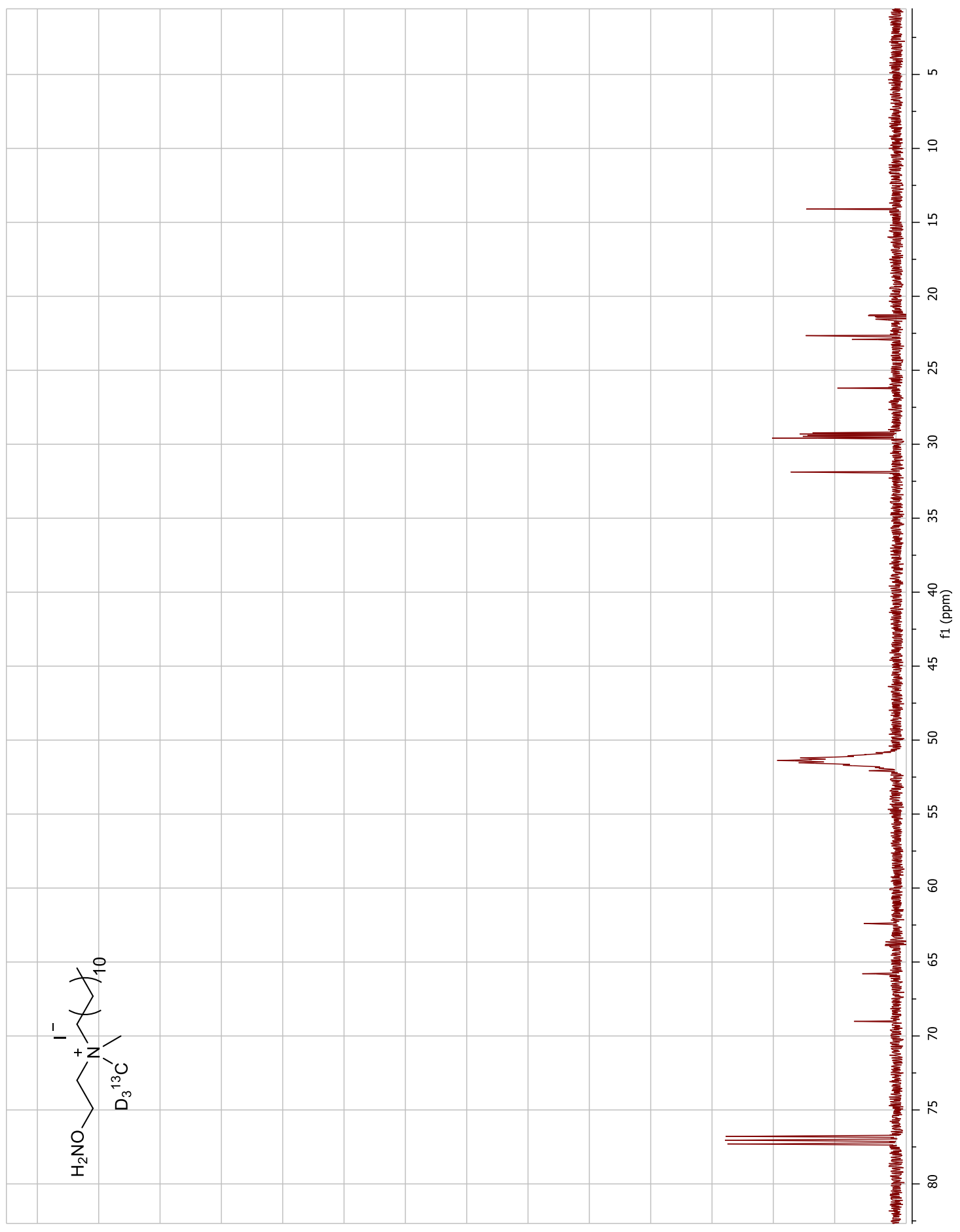

${ }^{13} \mathrm{C}$ NMR of *QDA $125 \mathrm{MHz} \mathrm{CDCl}_{3}$ 


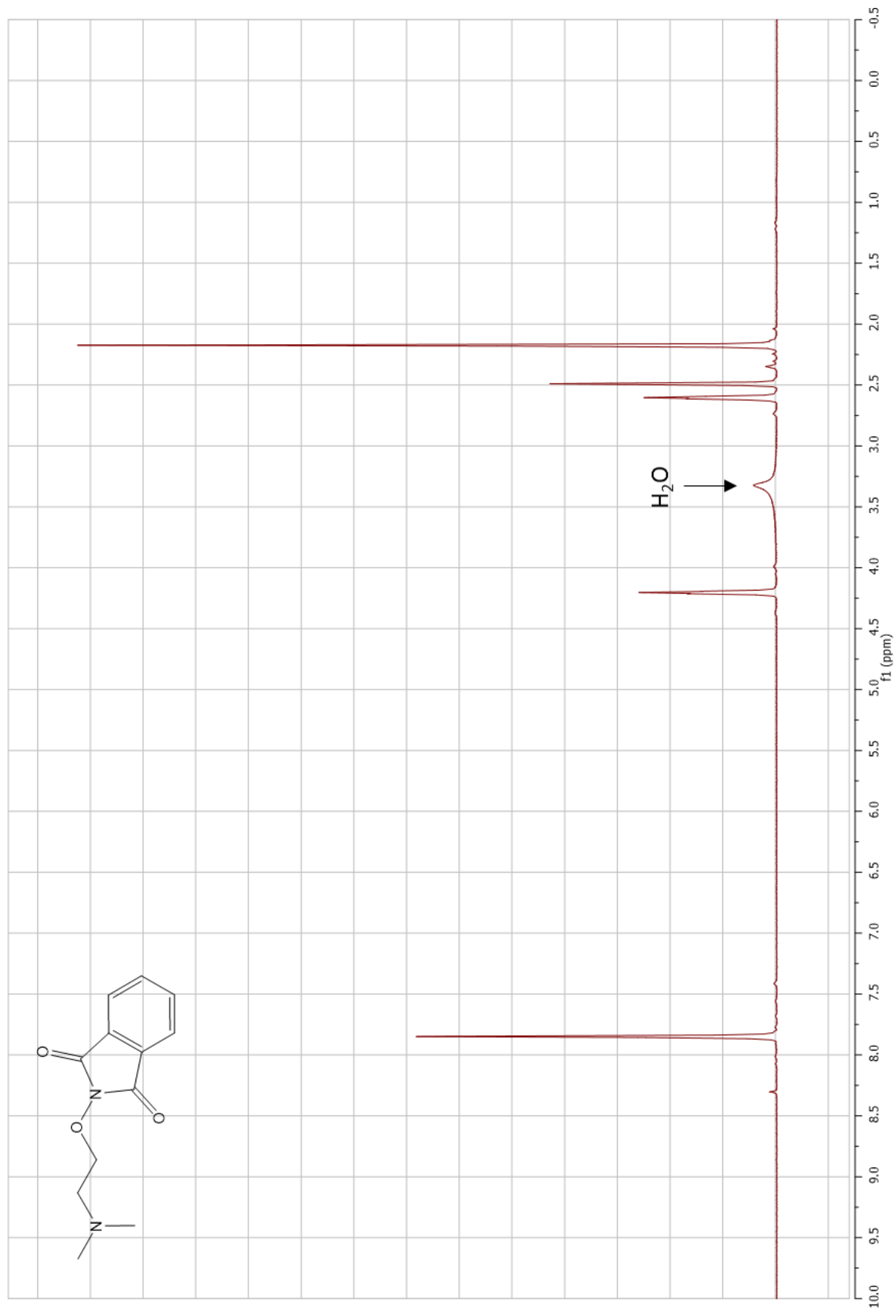

${ }^{1} \mathrm{H}$ NMR $500 \mathrm{MHz}$ DMSO 


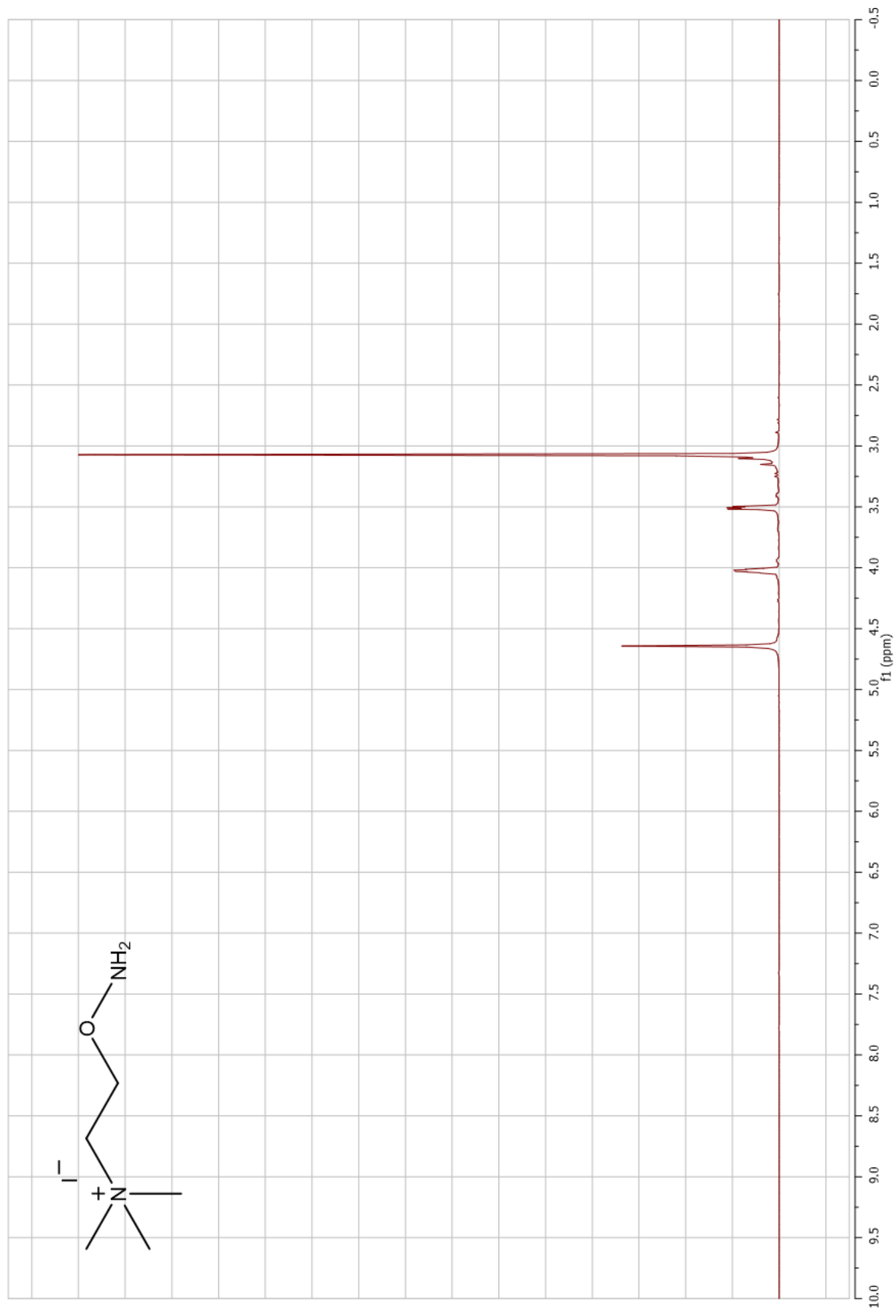

${ }^{1} \mathrm{H}$ NMR of QAO $400 \mathrm{MHz} \mathrm{D}_{2} \mathrm{O}$ 


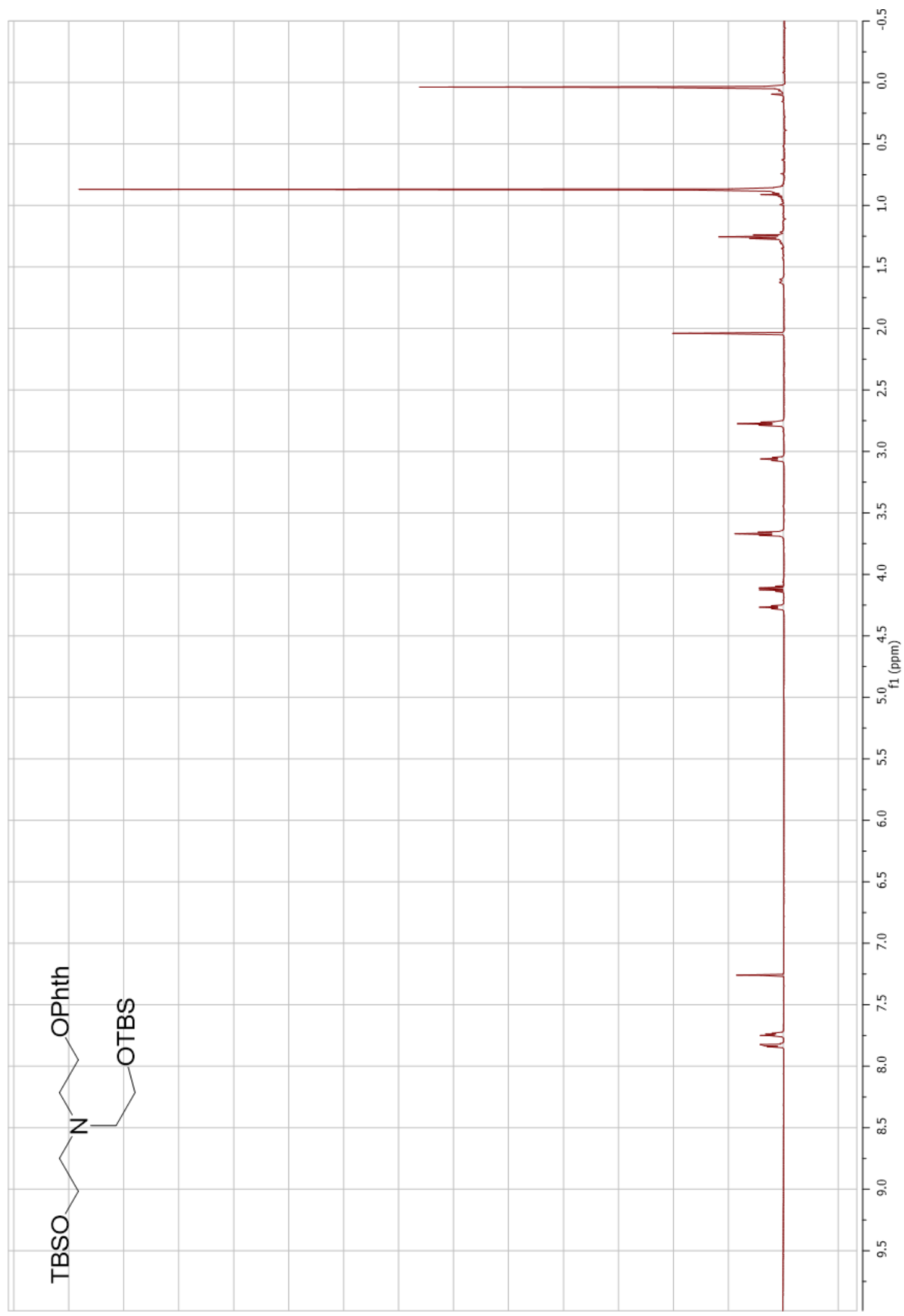

${ }^{1} \mathrm{H}$ NMR of $3.2500 \mathrm{MHz} \mathrm{CDCl}_{3}$ 


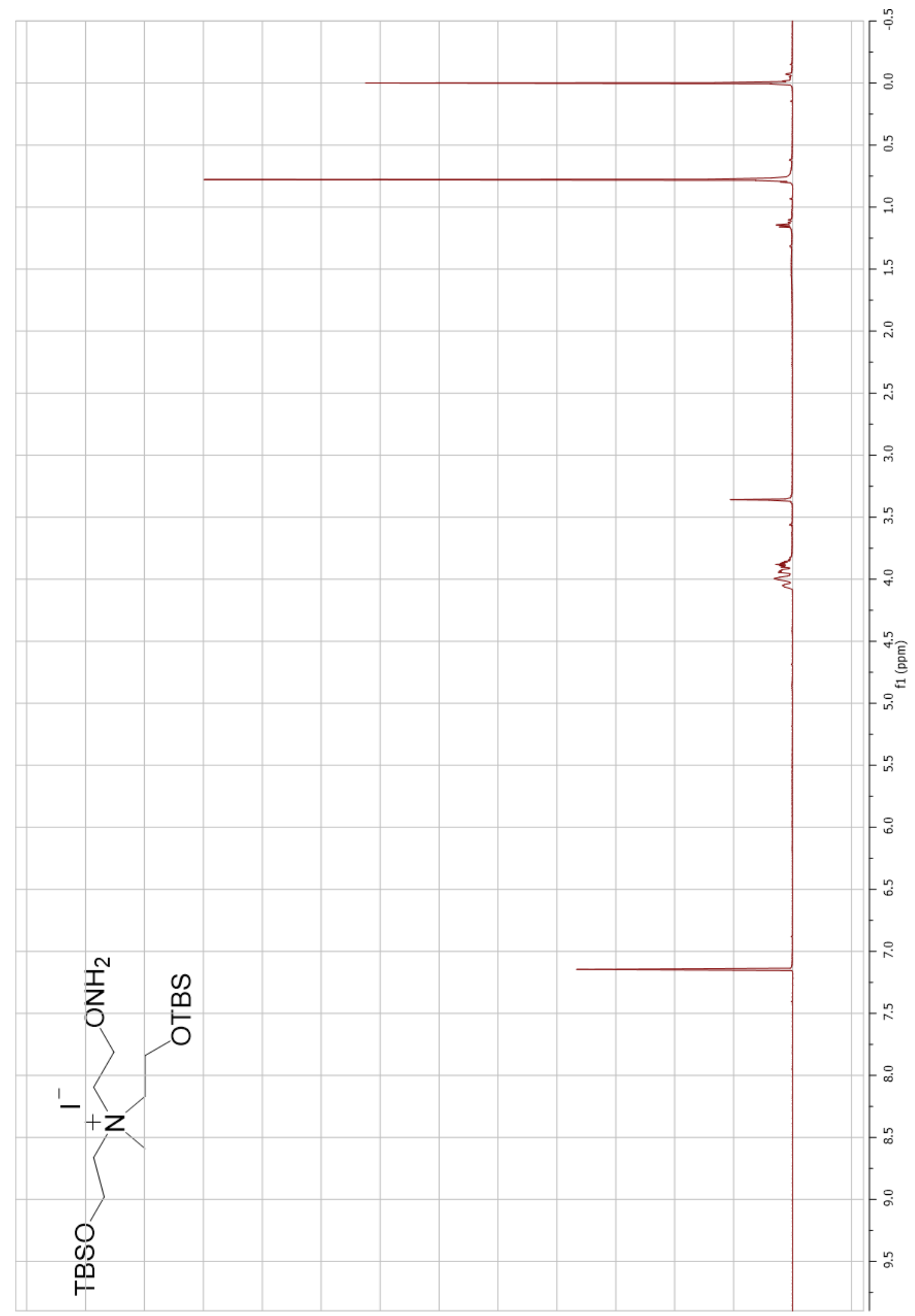

${ }^{1} \mathrm{H}$ NMR of $3.3400 \mathrm{MHz} \mathrm{CDCl}_{3}$ 


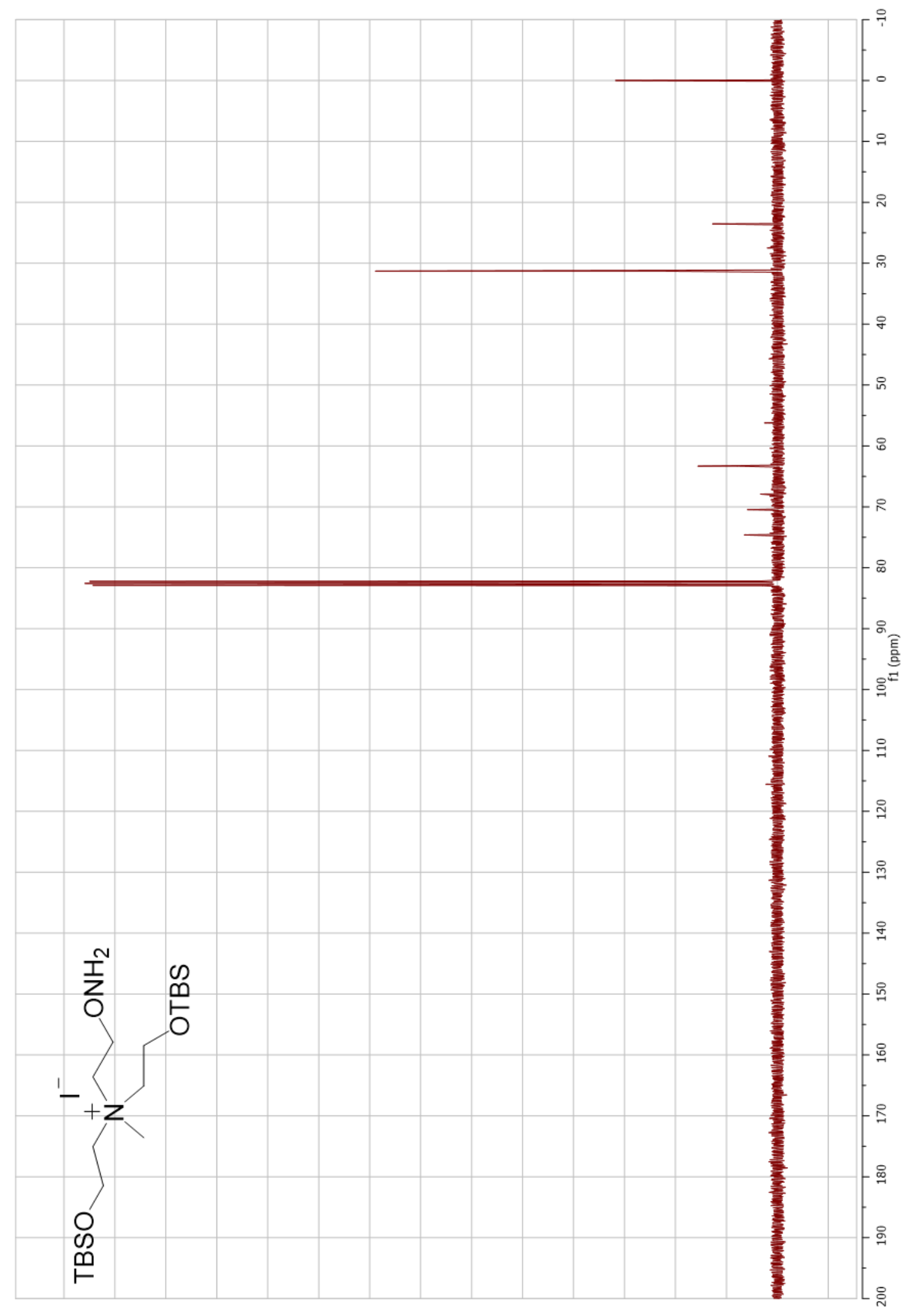

${ }^{13} \mathrm{C} \mathrm{NMR}$ of $3.3400 \mathrm{MHz}^{\mathrm{CDCl}} 3$ 


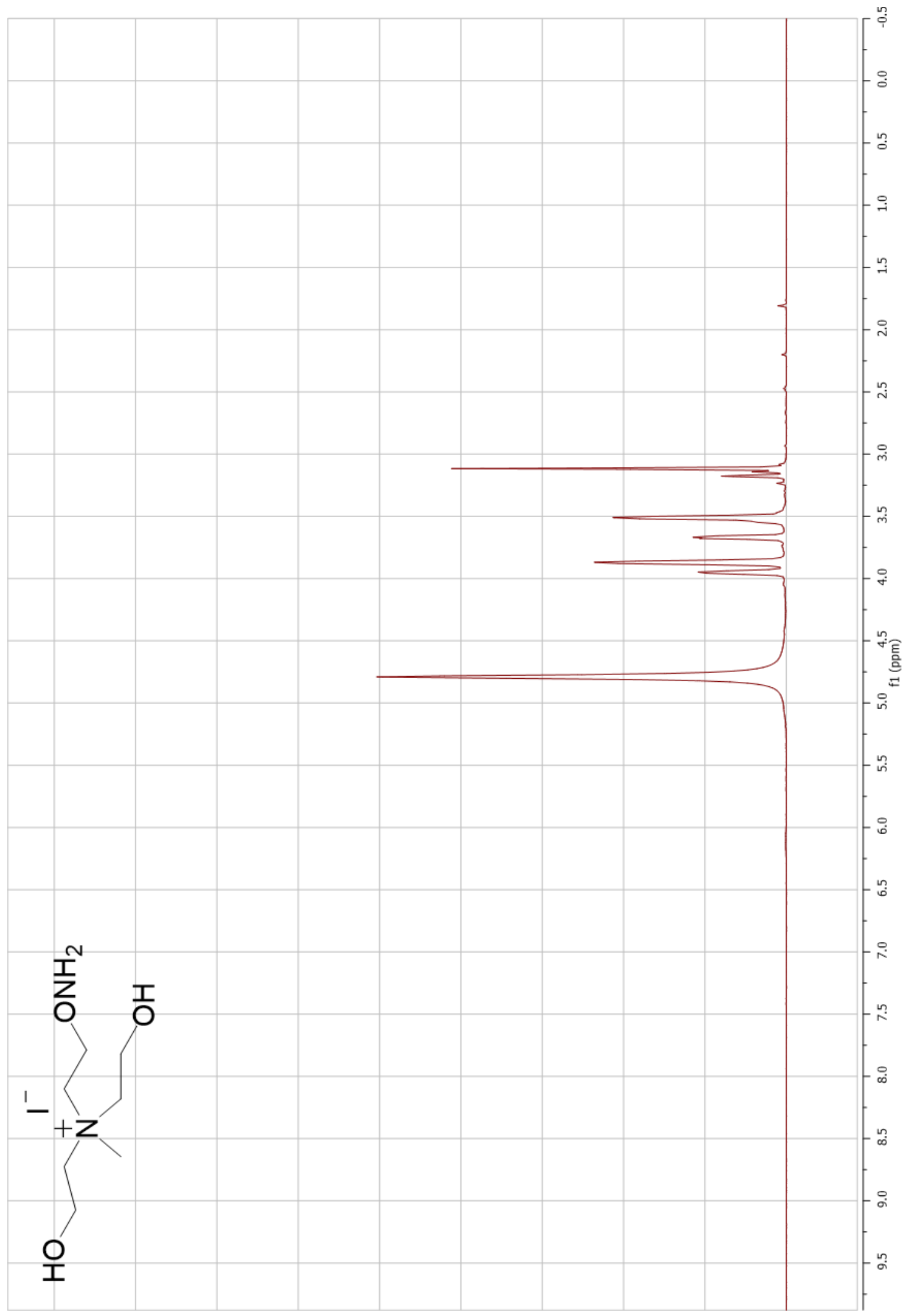

${ }^{1} \mathrm{H}$ NMR of QAO-2OH $400 \mathrm{MHz} \mathrm{D}_{2} \mathrm{O}$ 


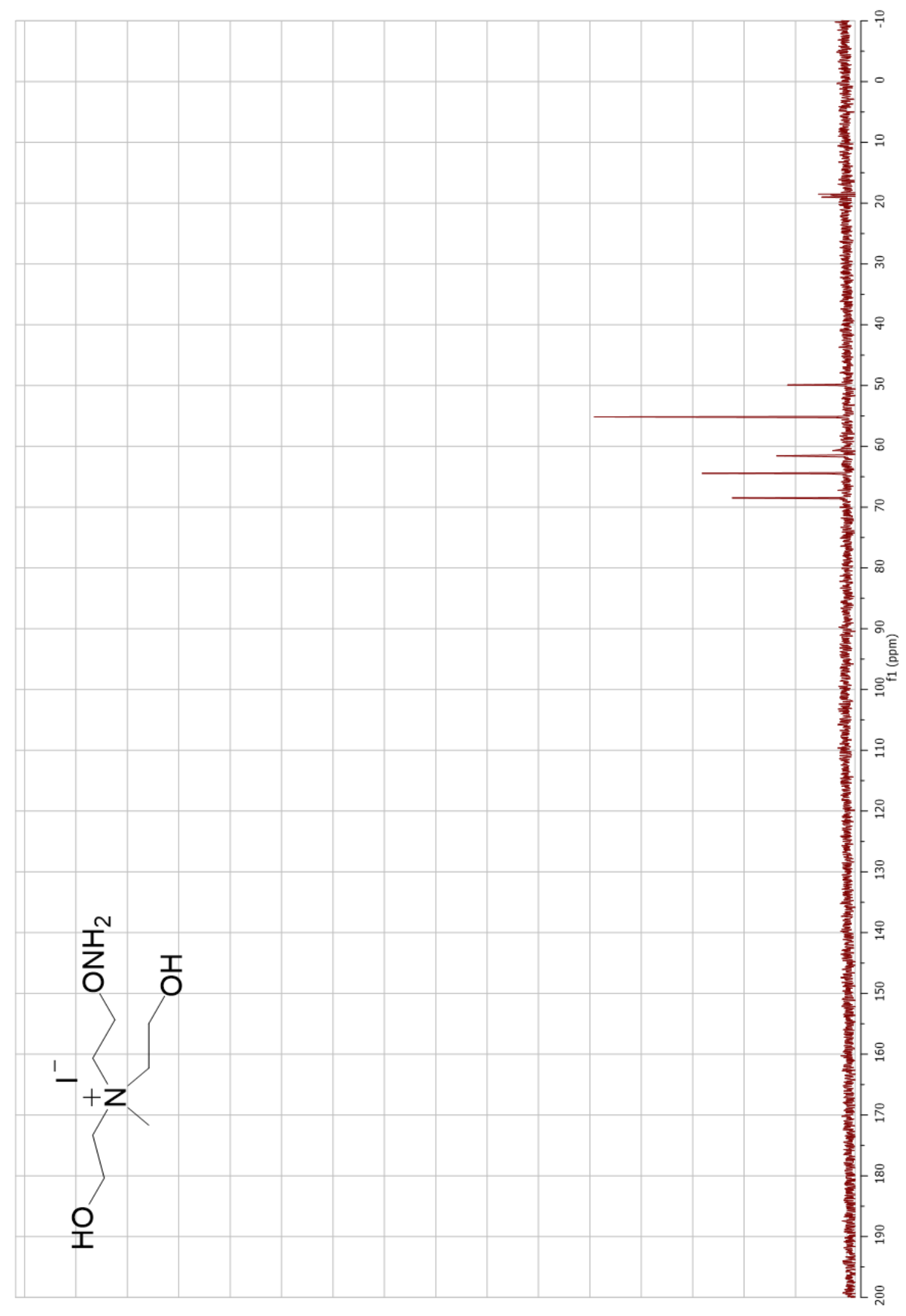

${ }^{13} \mathrm{C}$ NMR $125 \mathrm{MHz} \mathrm{D}_{2} \mathrm{O}$ 


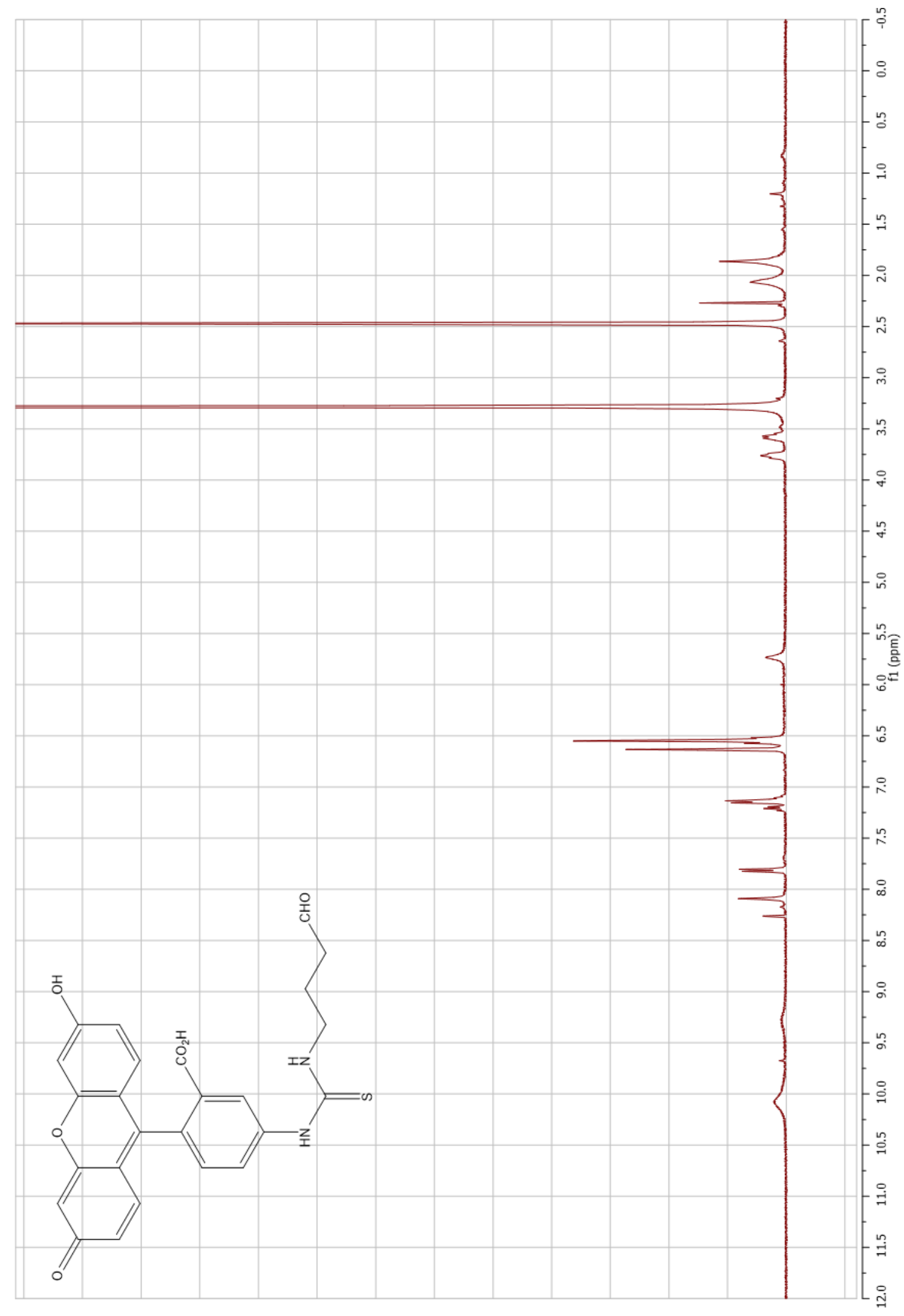

${ }^{1} \mathrm{H}$ NMR FITC-CHO $400 \mathrm{MHz}$ DMSO 


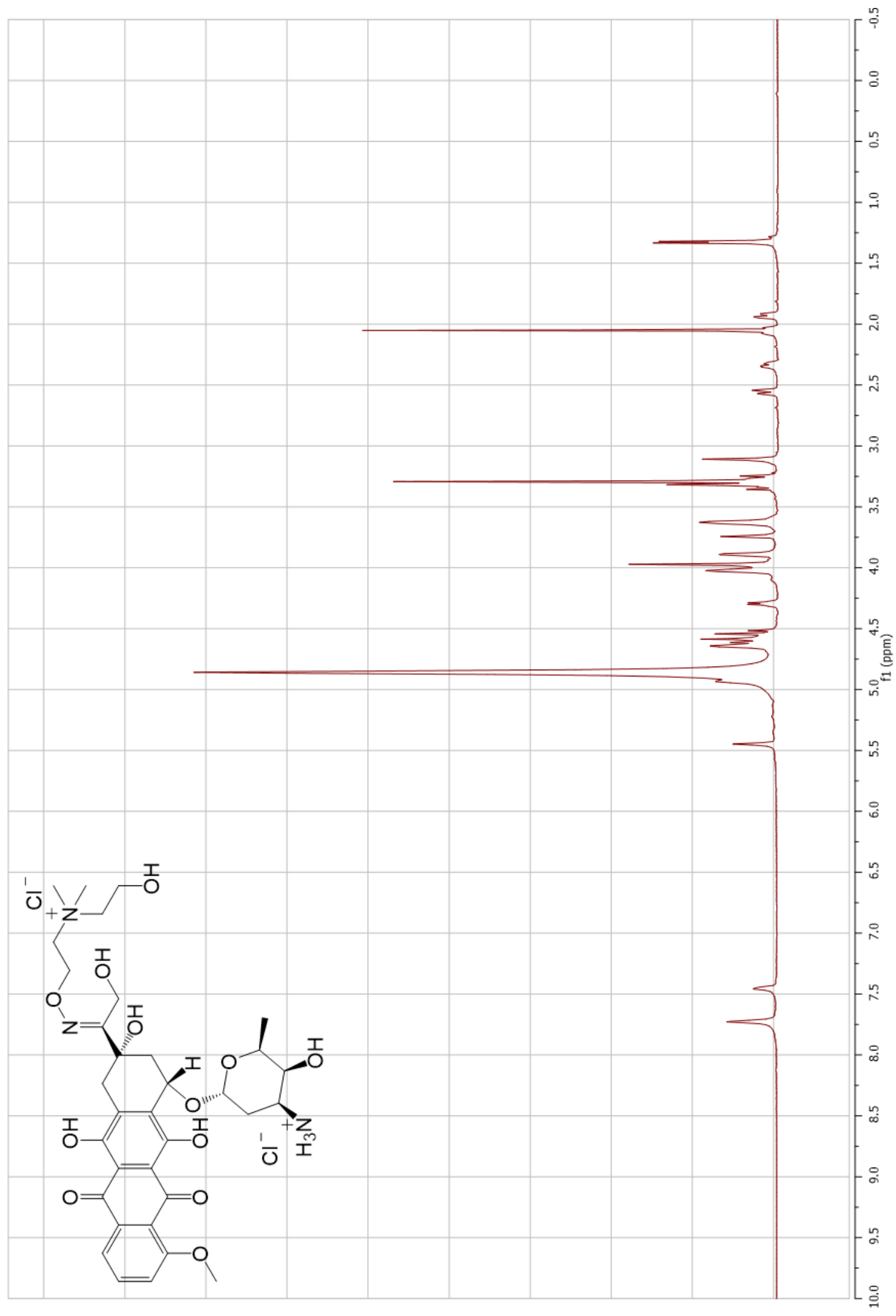

${ }^{1} \mathrm{H}$ NMR of Dox-OH $400 \mathrm{MHz}$ DMSO 


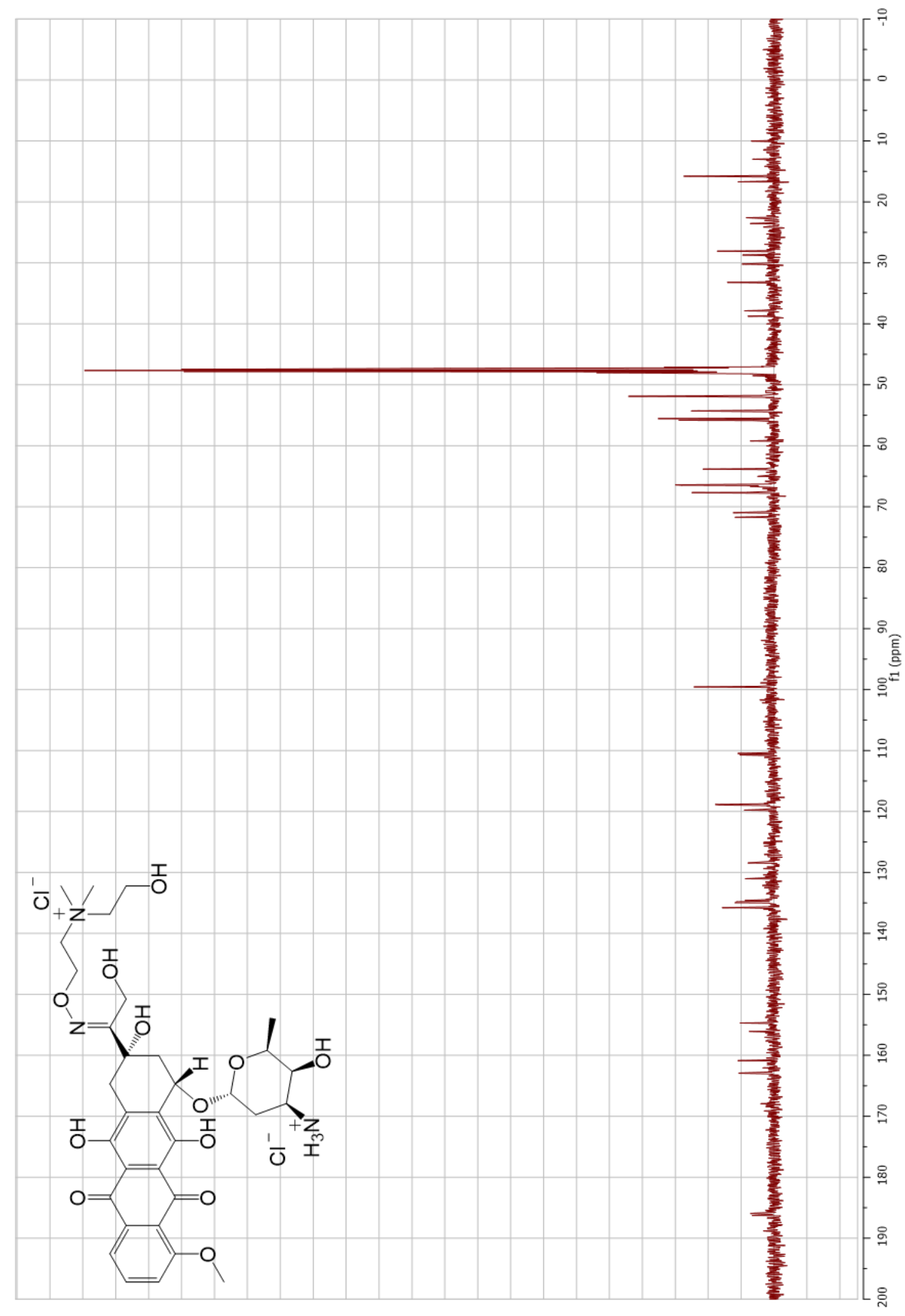

${ }^{13} \mathrm{C}$ NMR of Dox-OH $125 \mathrm{MHz} \mathrm{CD}_{3} \mathrm{OD}$ 


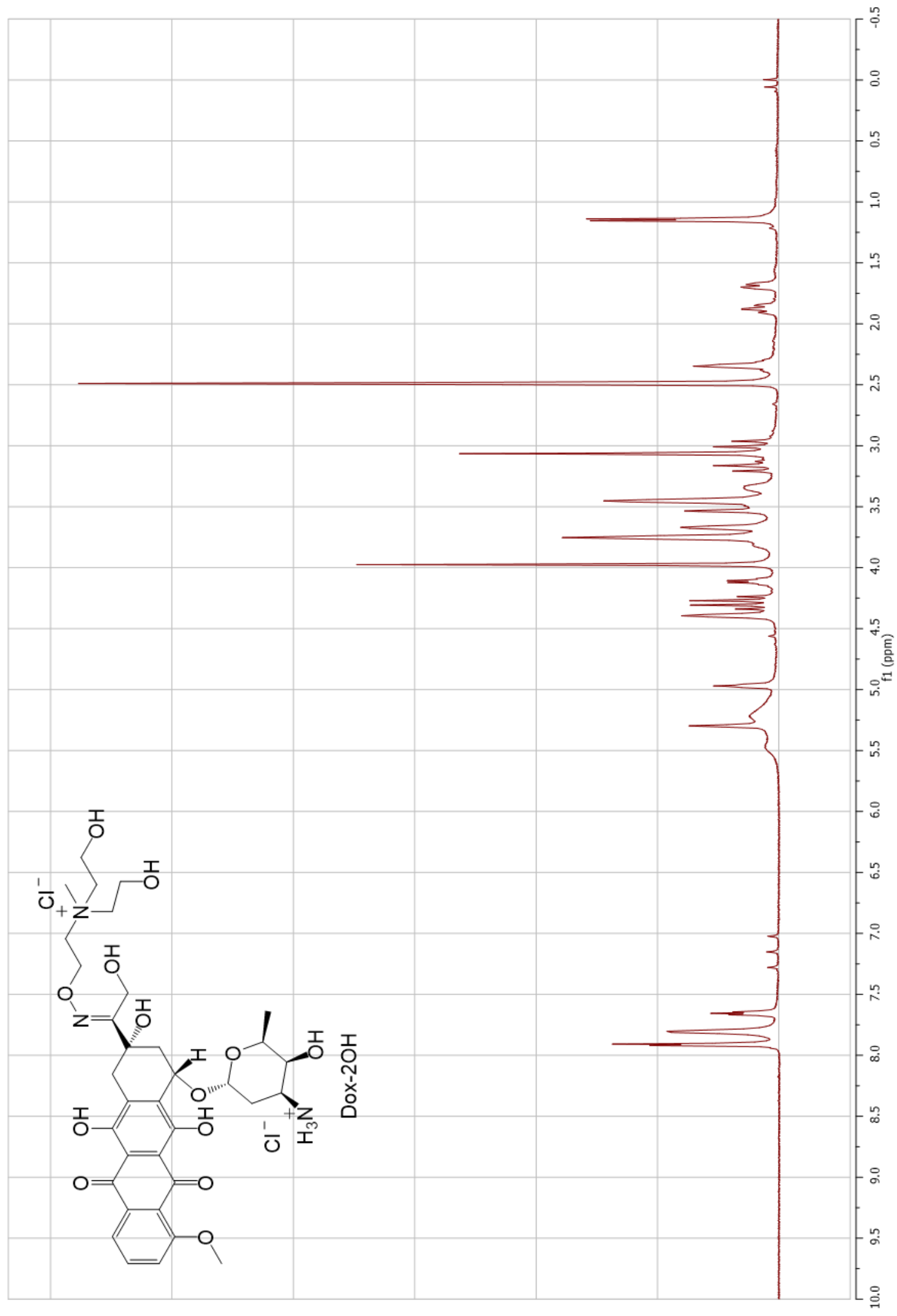

${ }^{1} \mathrm{H}$ NMR of Dox-2OH $400 \mathrm{MHz}$ DMSO 


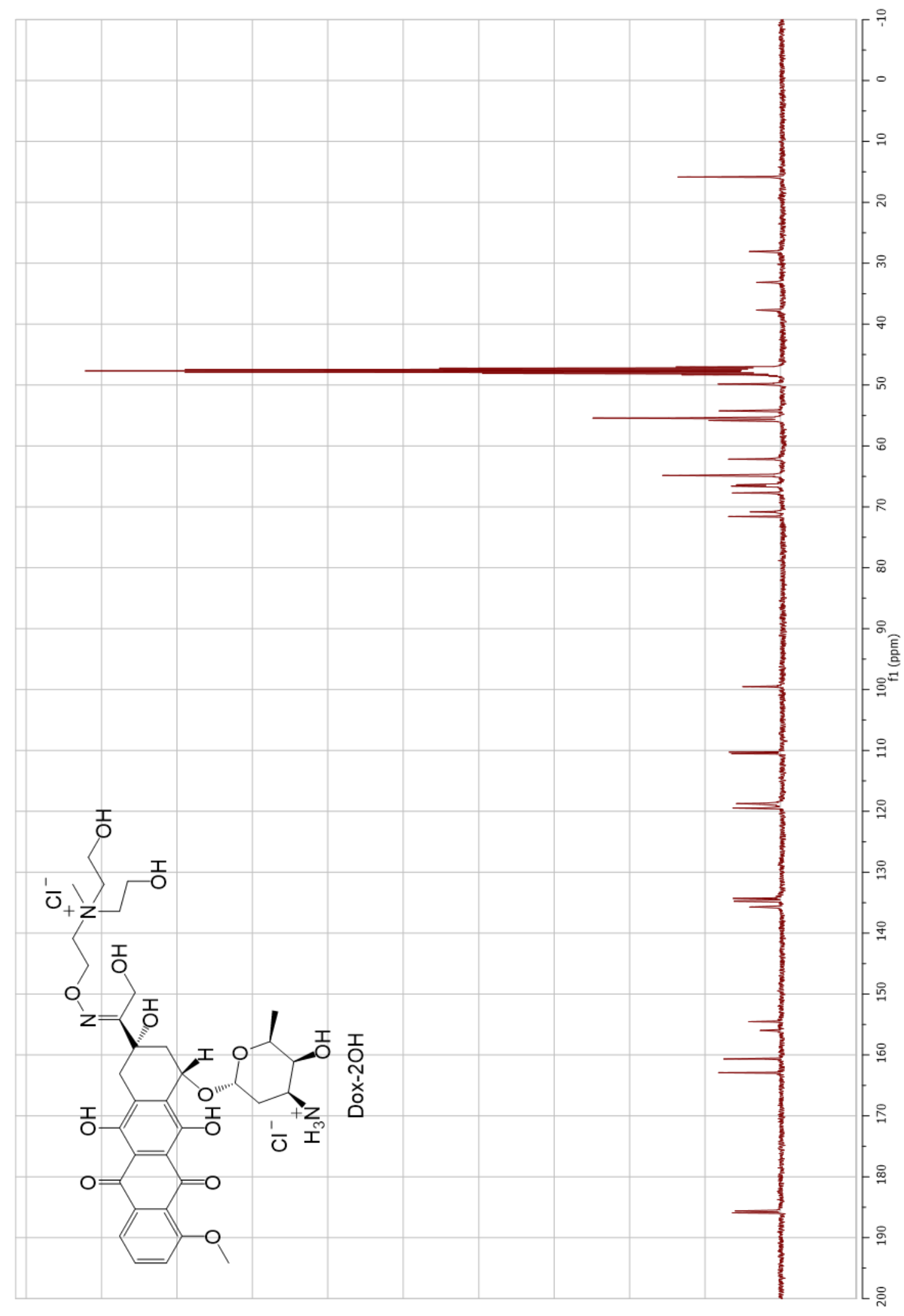

${ }^{13} \mathrm{C}$ NMR of Dox-2OH $100 \mathrm{MHz} \mathrm{CD}_{3} \mathrm{OD}$ 


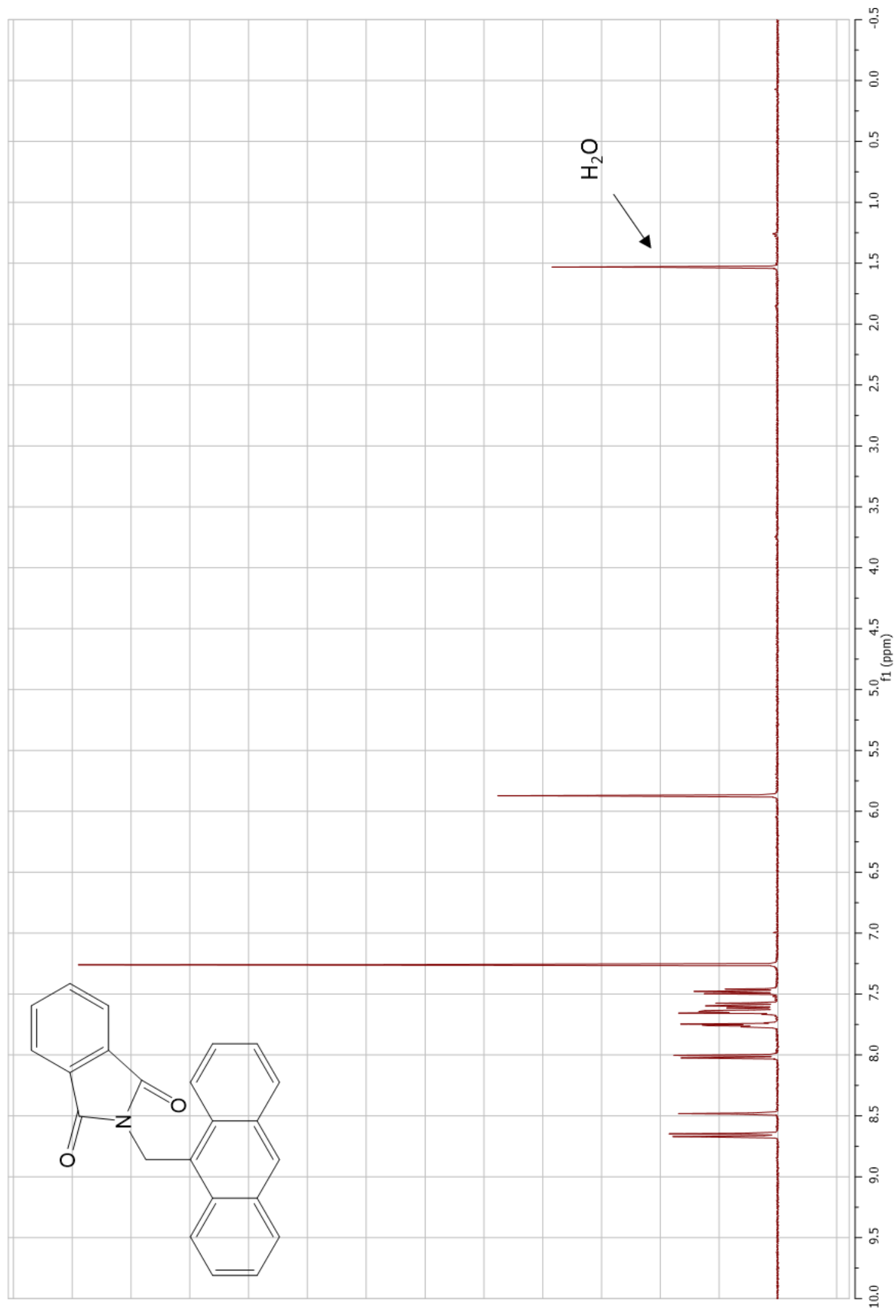

${ }^{1} \mathrm{H}$ NMR $400 \mathrm{MHz}^{\mathrm{CDCl}}{ }_{3}$ 


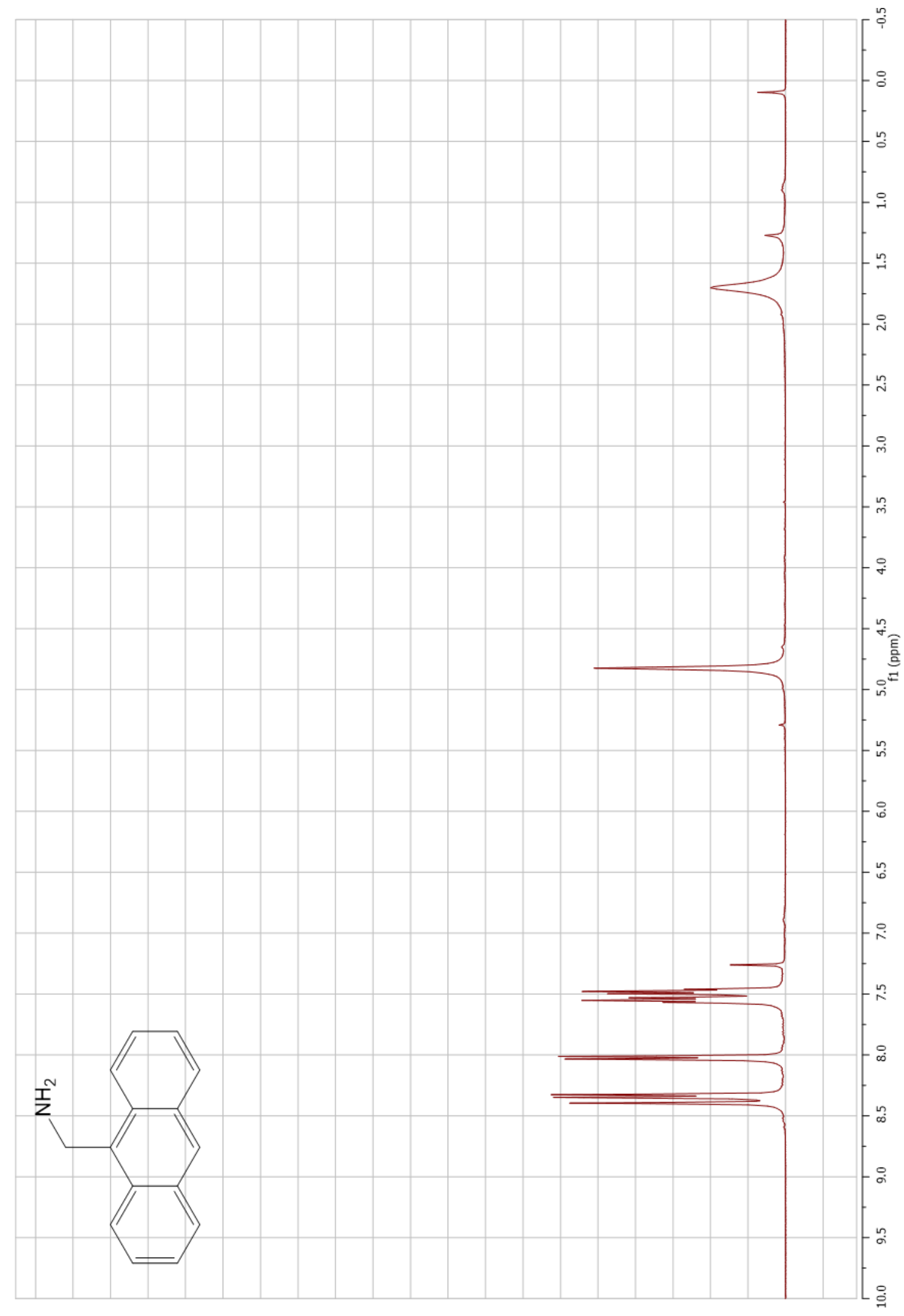

${ }^{1} \mathrm{H} \mathrm{NMR} 400 \mathrm{MHz} \mathrm{CDCl}_{3}$ 


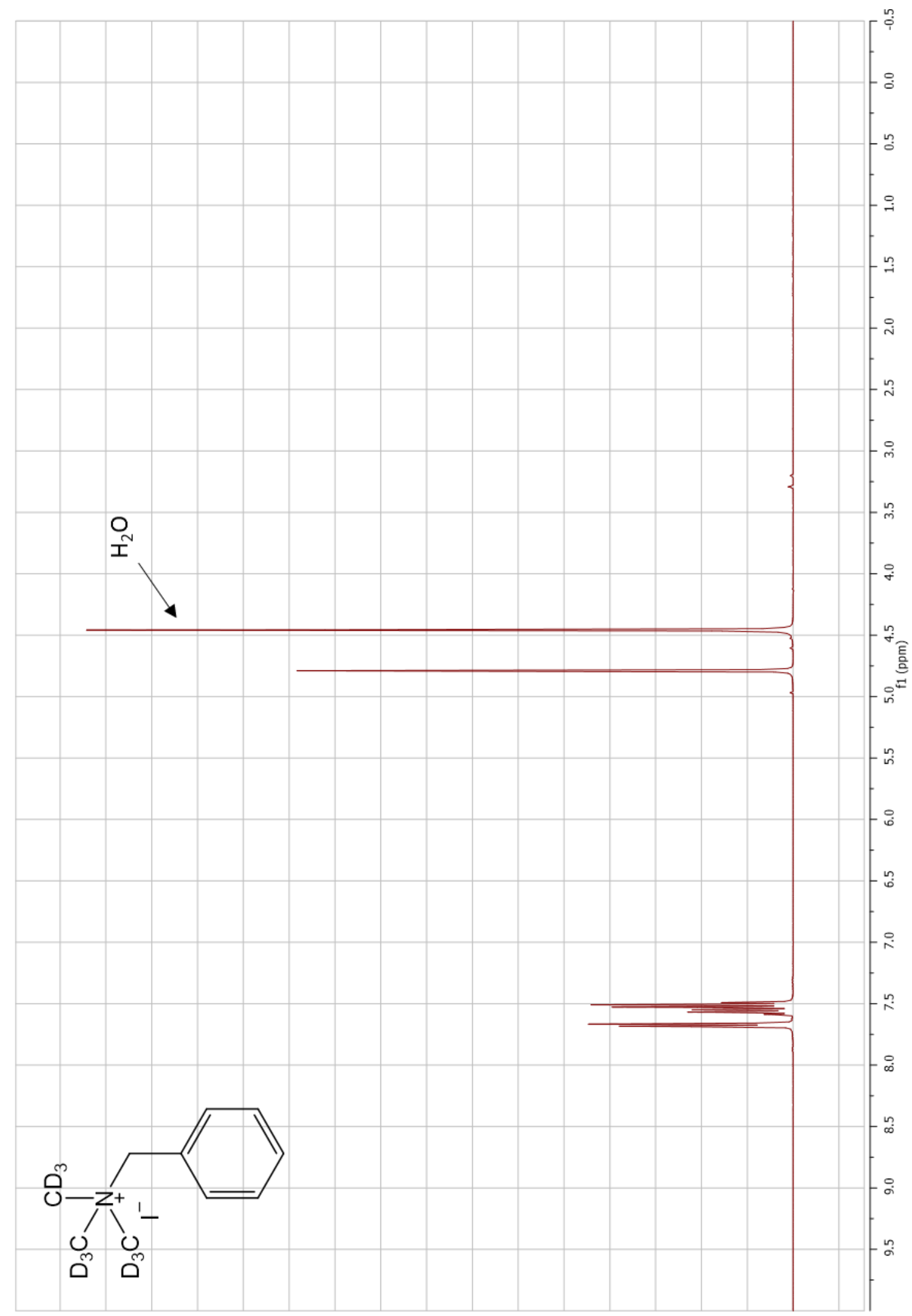

${ }^{1} \mathrm{H}$ NMR $400 \mathrm{MHz} \mathrm{D}_{2} \mathrm{O}$ 


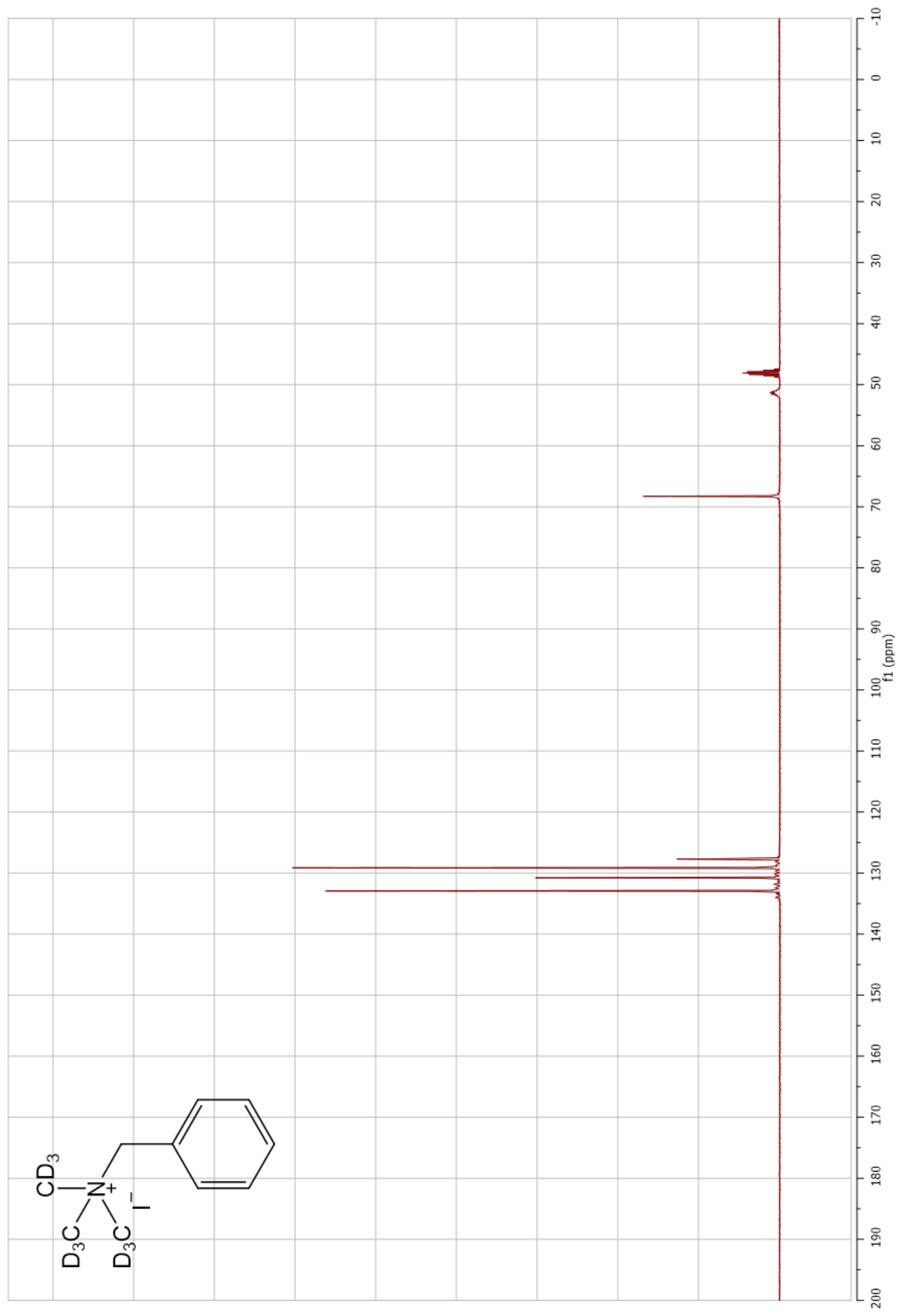

${ }^{13} \mathrm{C}$ NMR $100 \mathrm{MHz} \mathrm{CD}_{3} \mathrm{OD}$ 


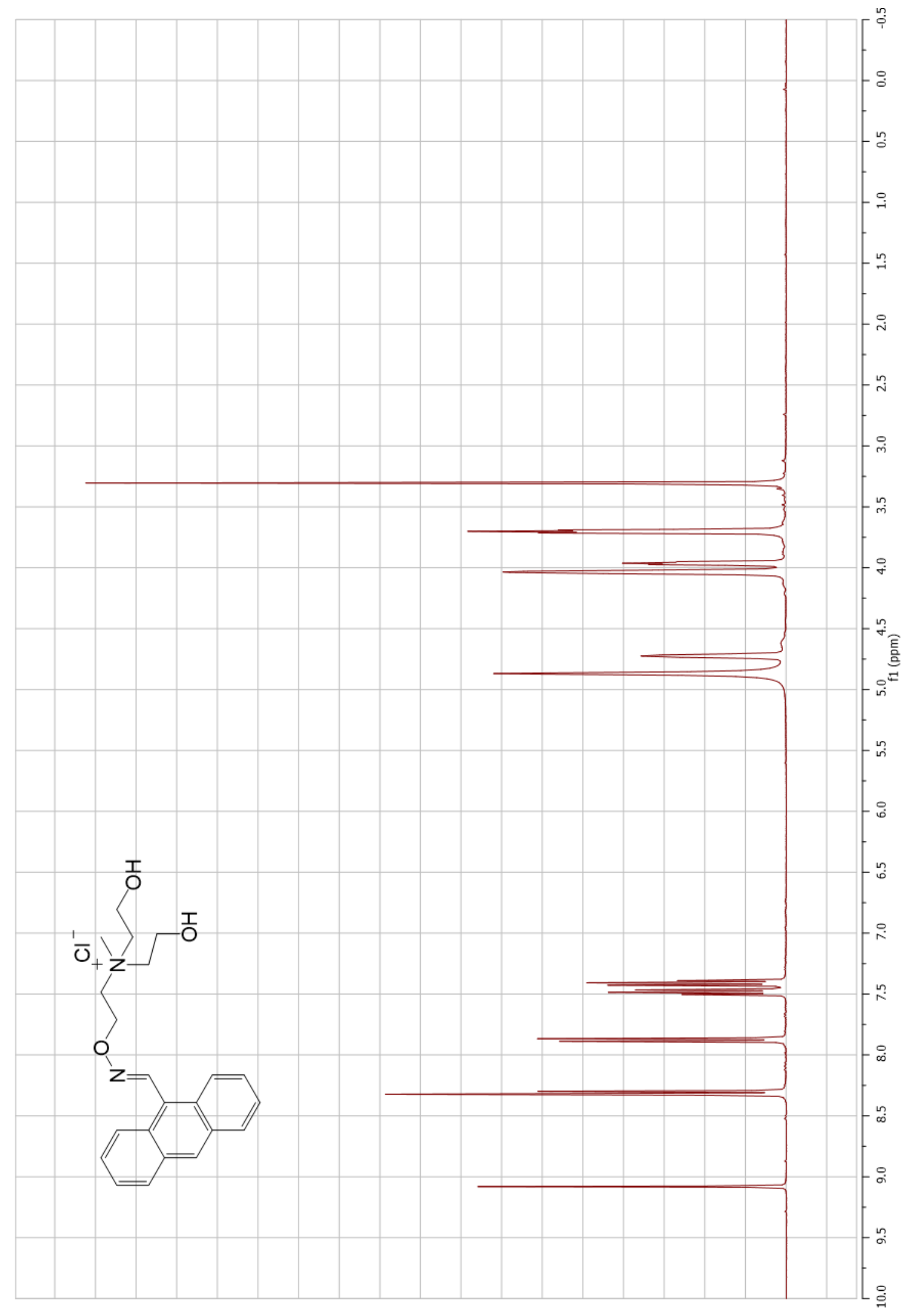

${ }^{1} \mathrm{H}$ NMR of QAO-2OH anthracene $400 \mathrm{MHz} \mathrm{CD}_{3} \mathrm{OD}$ 


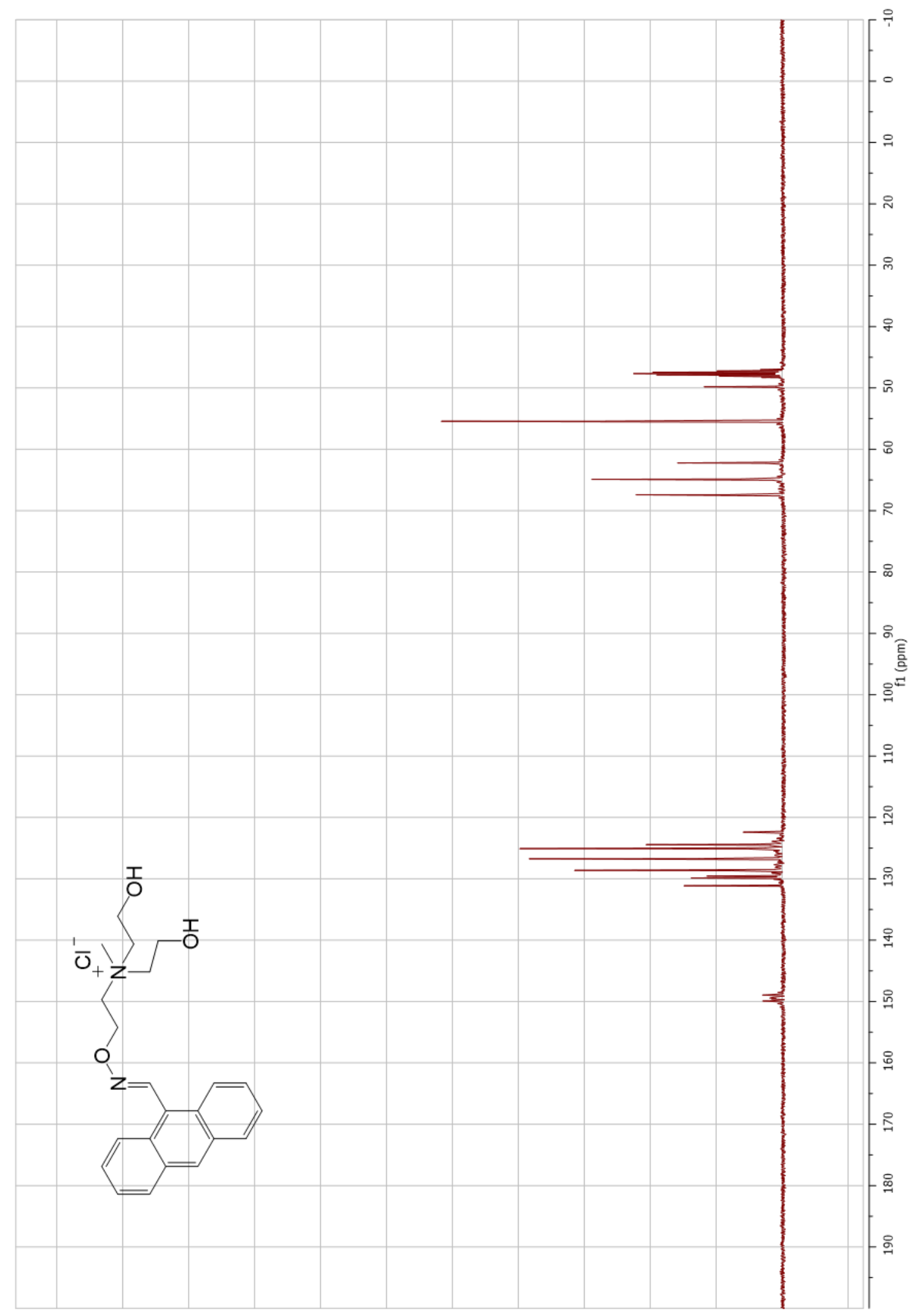

${ }^{13} \mathrm{C}$ NMR of QAO-2OH anthracene $100 \mathrm{MHz} \mathrm{CD}_{3} \mathrm{OD}$ 


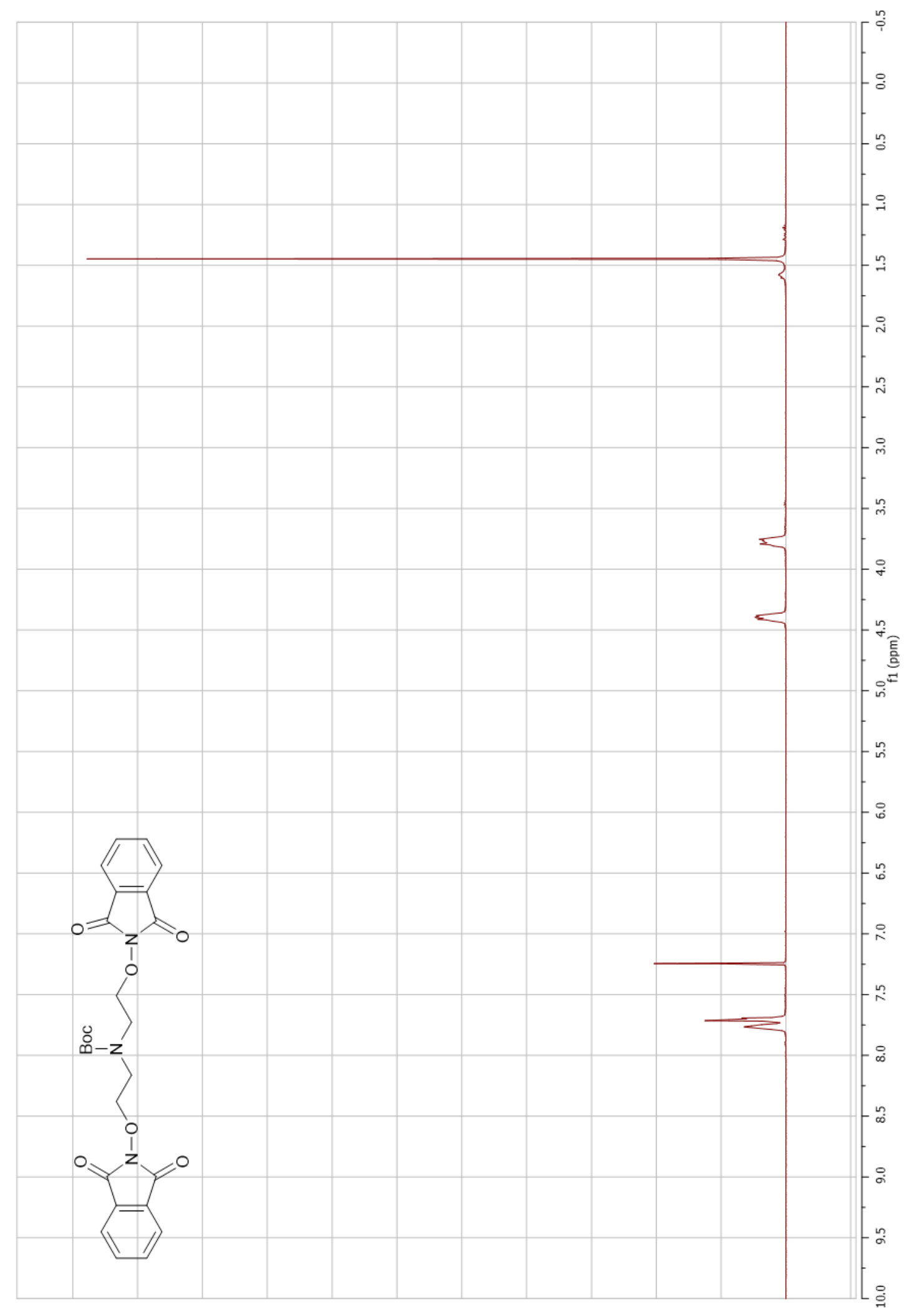

${ }^{1} \mathrm{H} \mathrm{NMR} 400 \mathrm{MHz} \mathrm{CDCl}_{3}$ 


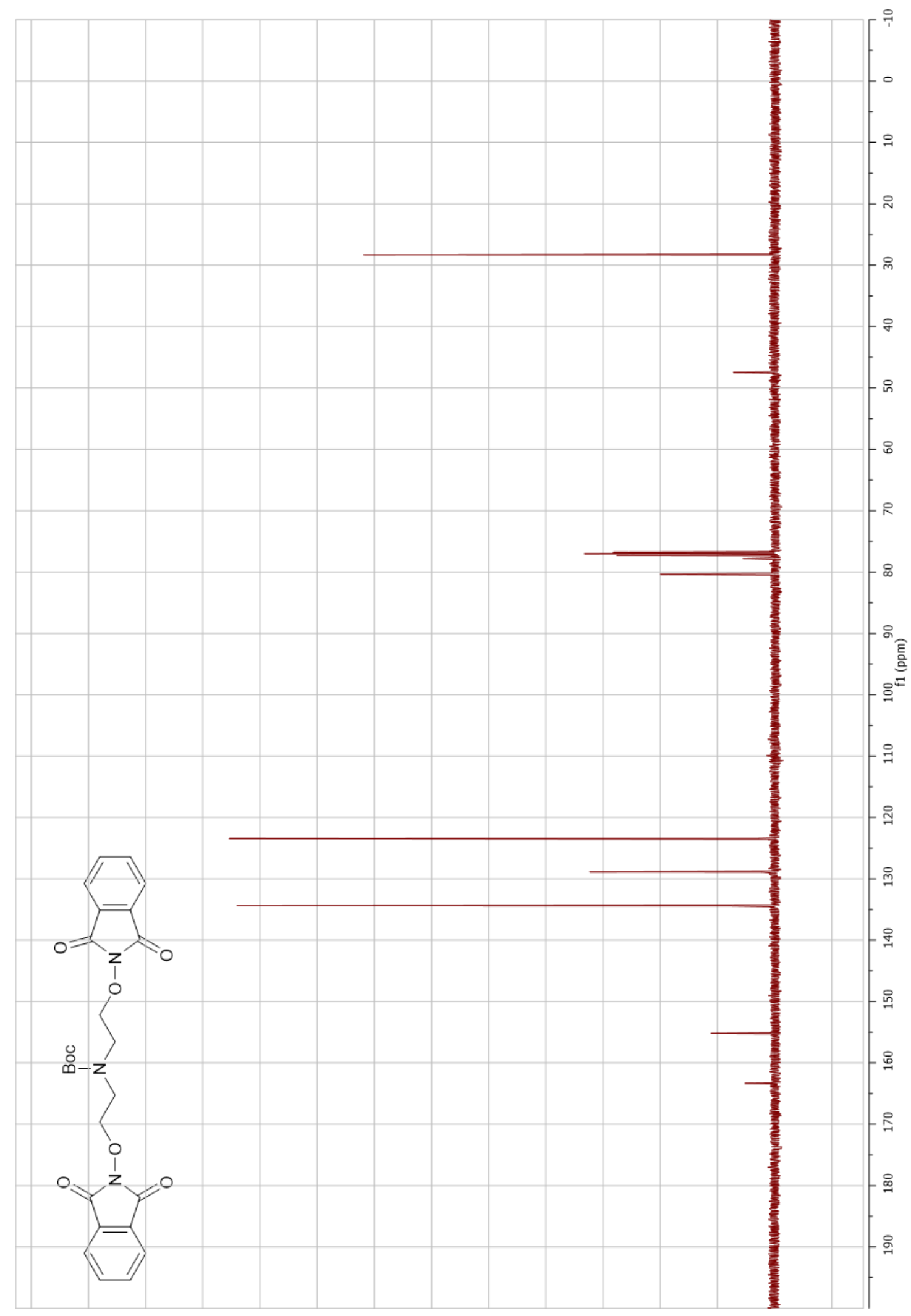

${ }^{13} \mathrm{C}$ NMR of $100 \mathrm{MHz}^{\mathrm{CDCl}} 3$ 


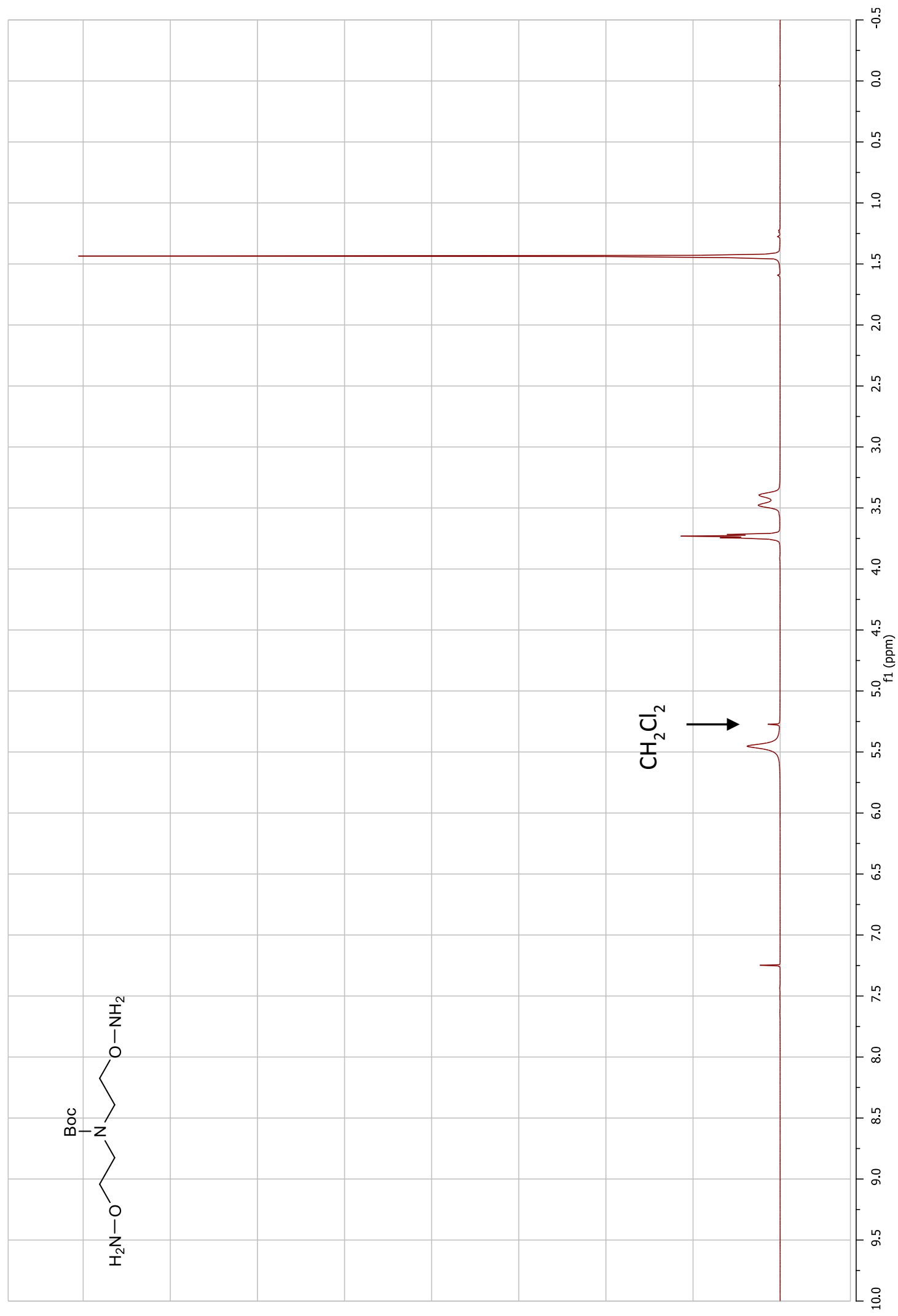

${ }^{1} \mathrm{H}$ NMR of $4.1400 \mathrm{MHz} \mathrm{CDCl}_{3}$ 


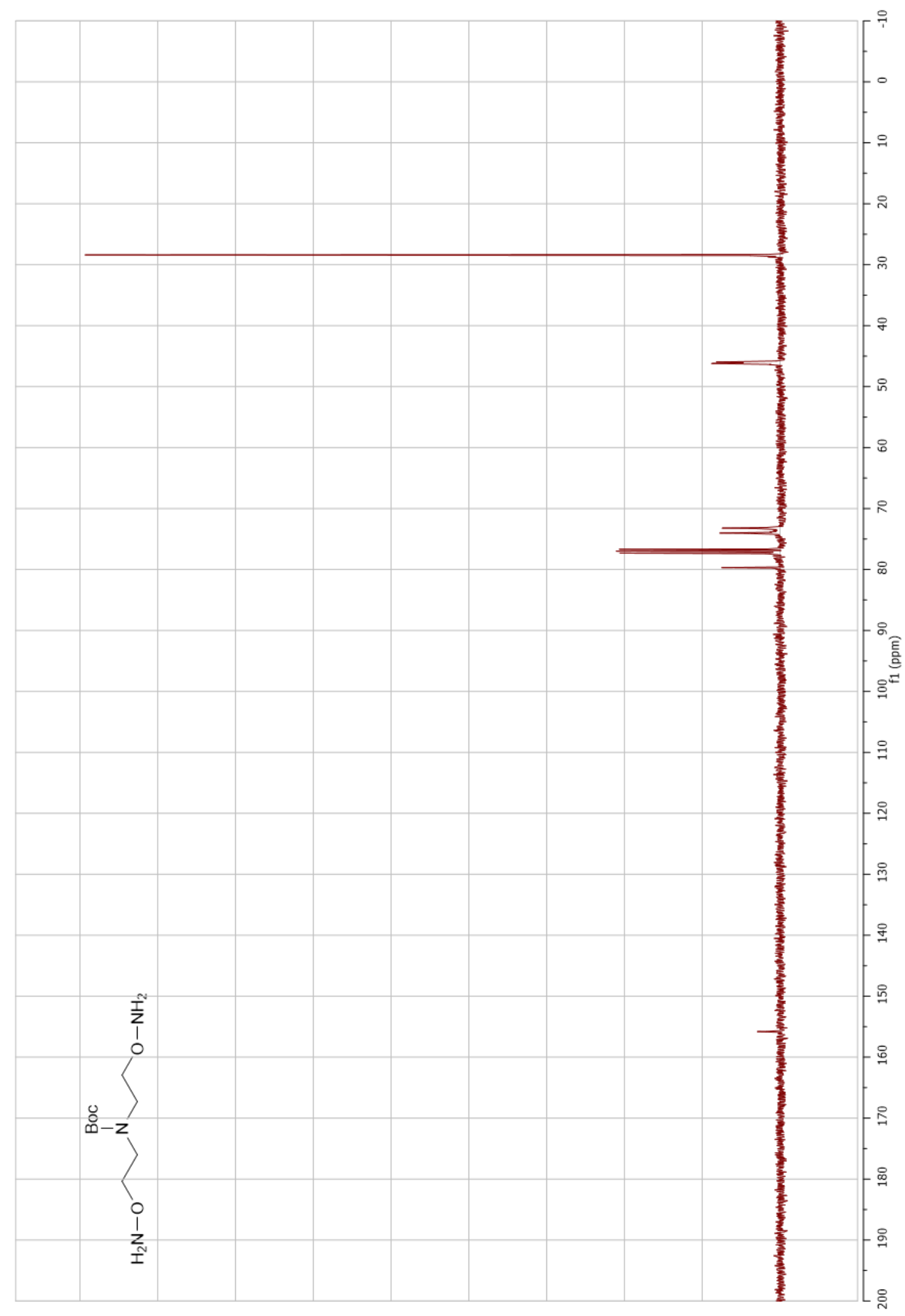

${ }^{13} \mathrm{C} \mathrm{NMR}$ of $4.1100 \mathrm{MHz} \mathrm{CDCl}_{3}$ 


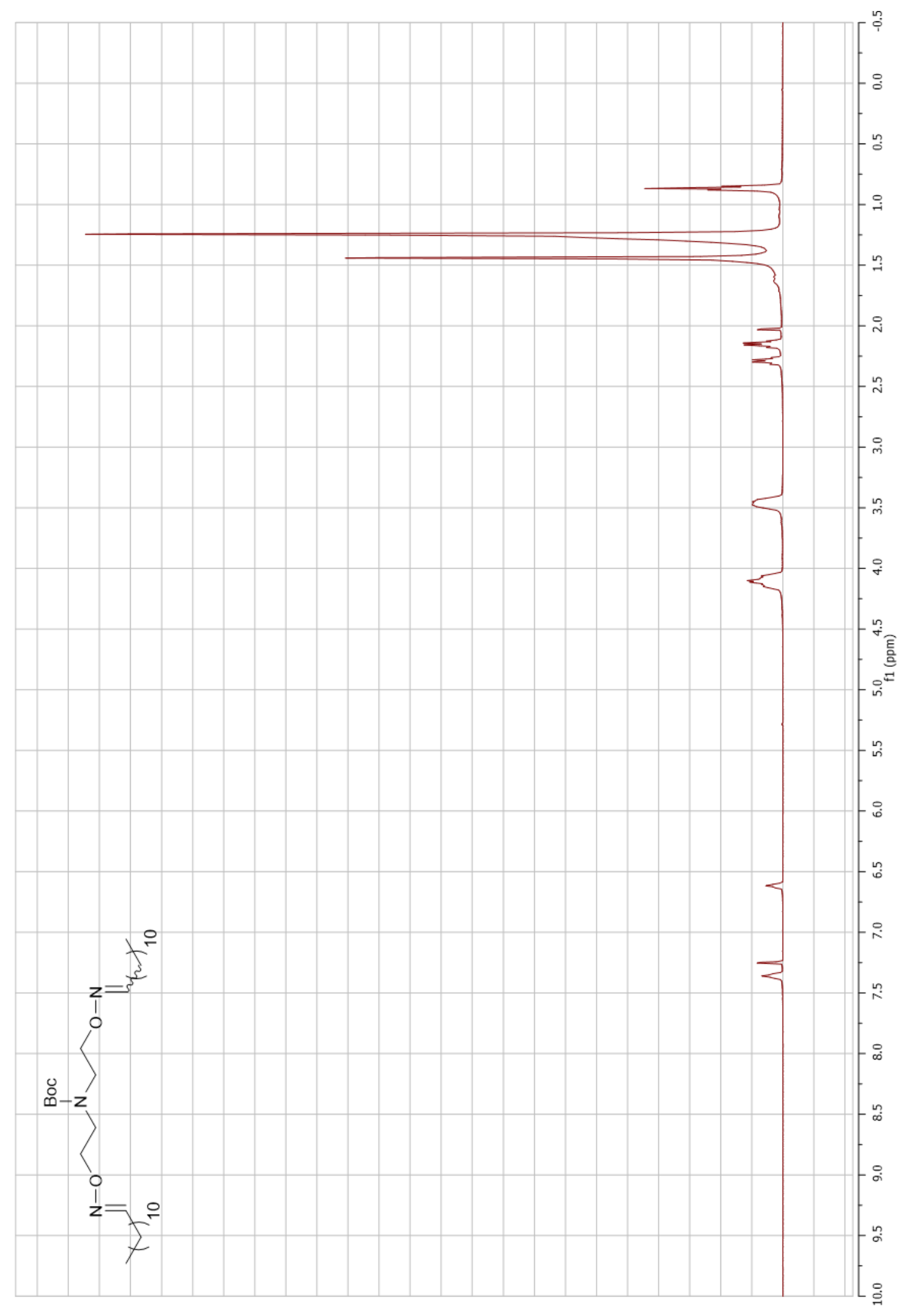

${ }^{1} \mathrm{H}$ NMR of $4.2400 \mathrm{MHz} \mathrm{CDCl}_{3}$ 


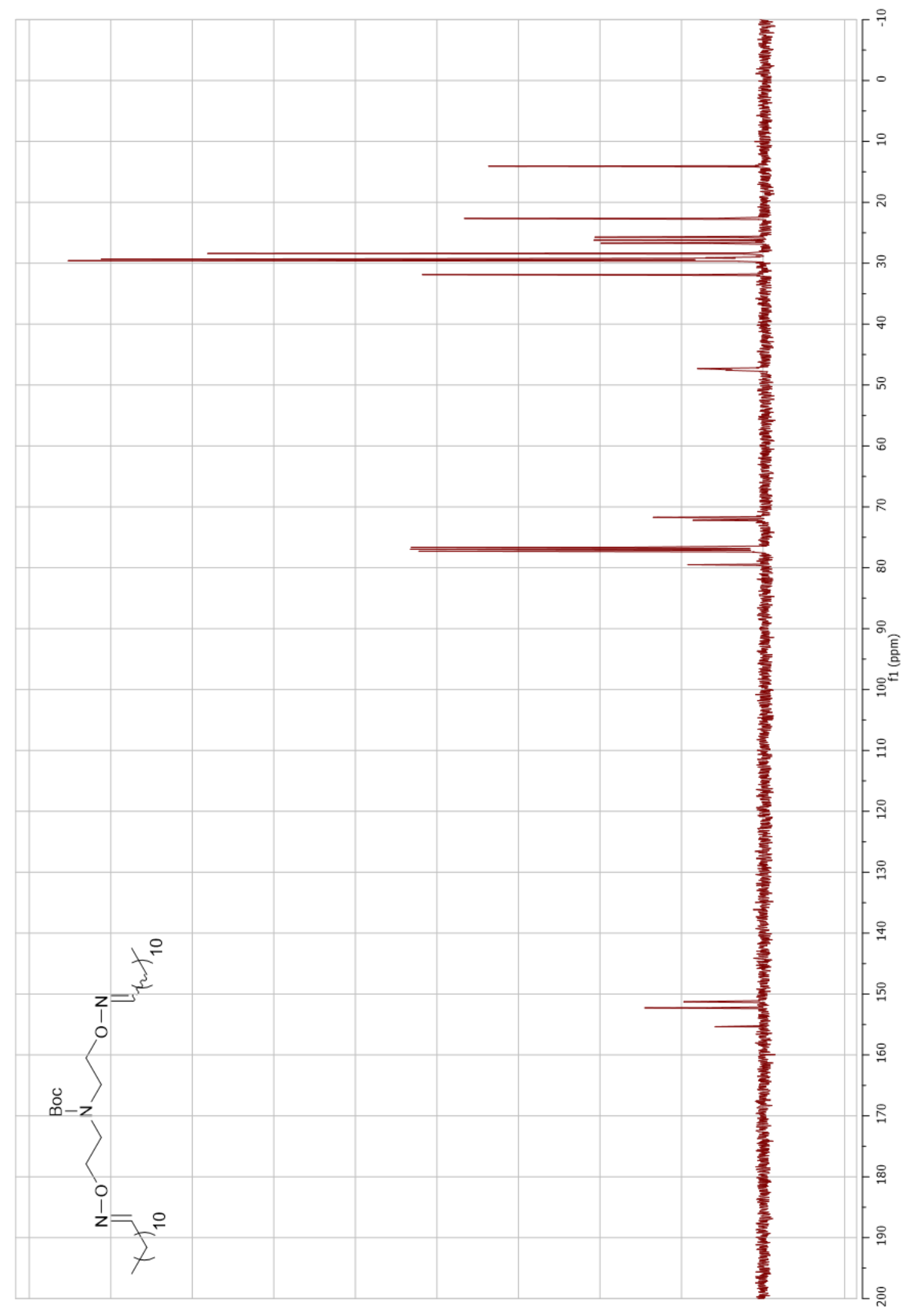

${ }^{13} \mathrm{C} \mathrm{NMR}$ of $4.2100 \mathrm{MHz} \mathrm{CDCl}_{3}$ 


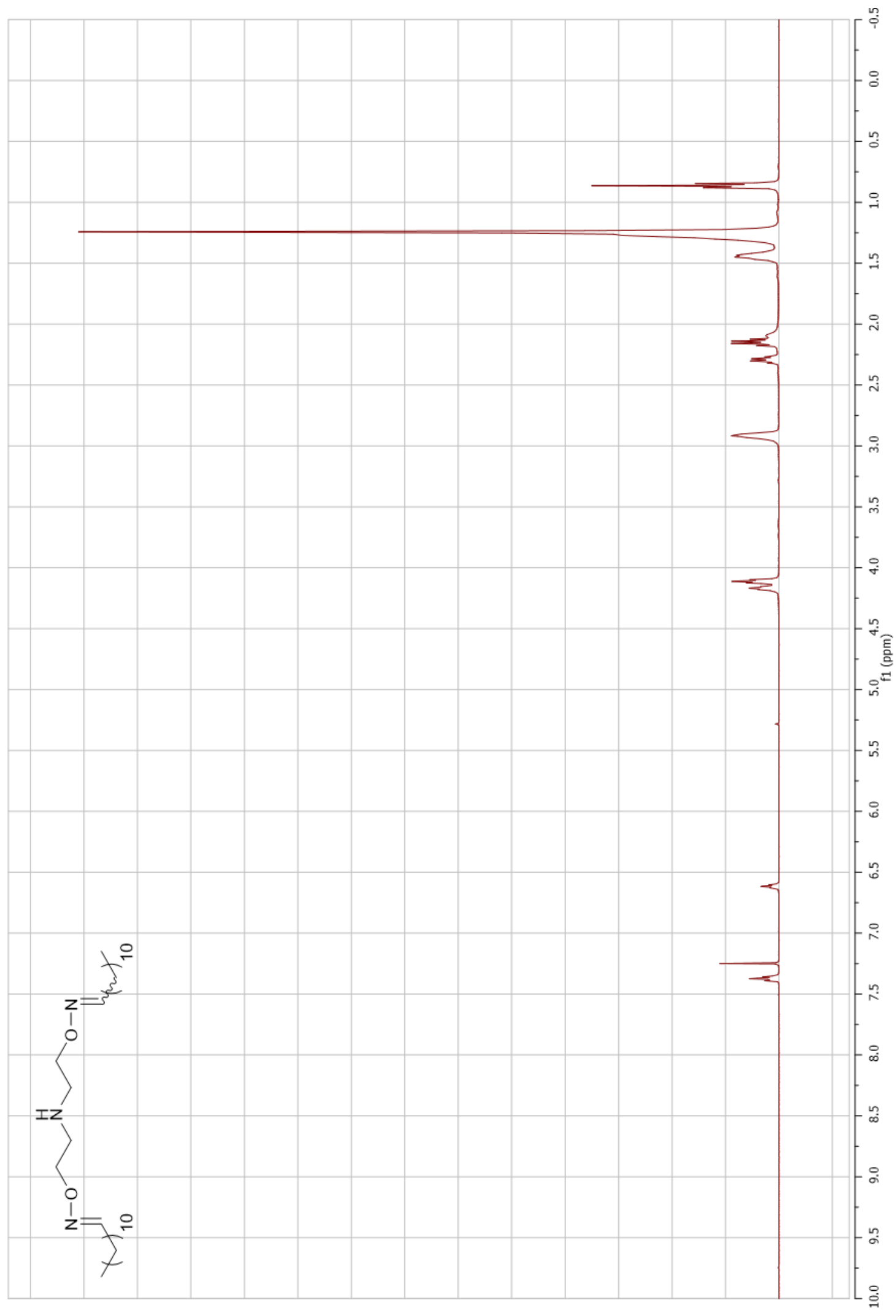

${ }^{1} \mathrm{H}$ NMR $400 \mathrm{MHz}^{\mathrm{CDCl}}{ }_{3}$ 


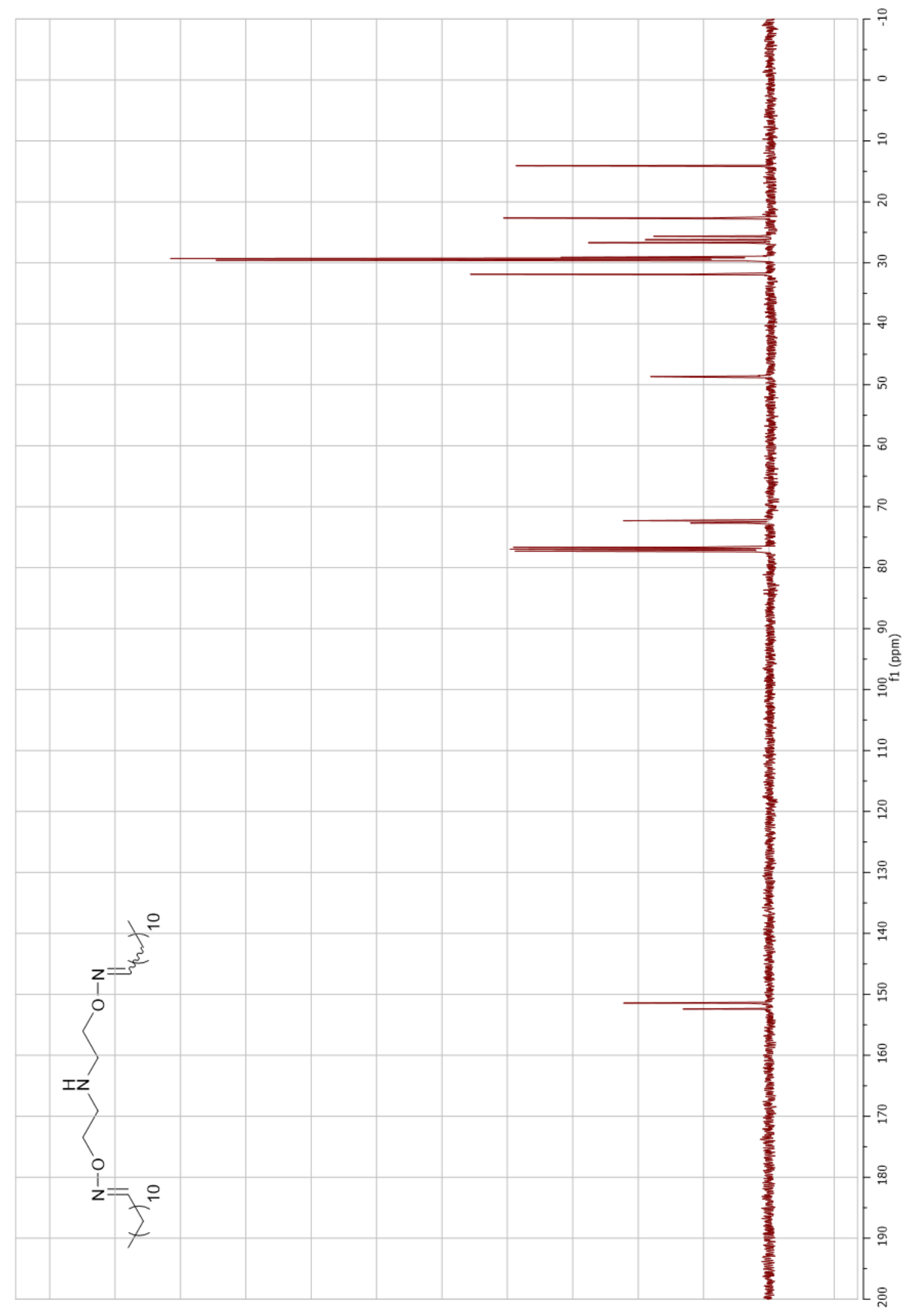

${ }^{13} \mathrm{C}$ NMR $100 \mathrm{MHz}^{\mathrm{CDCl}} 3$ 


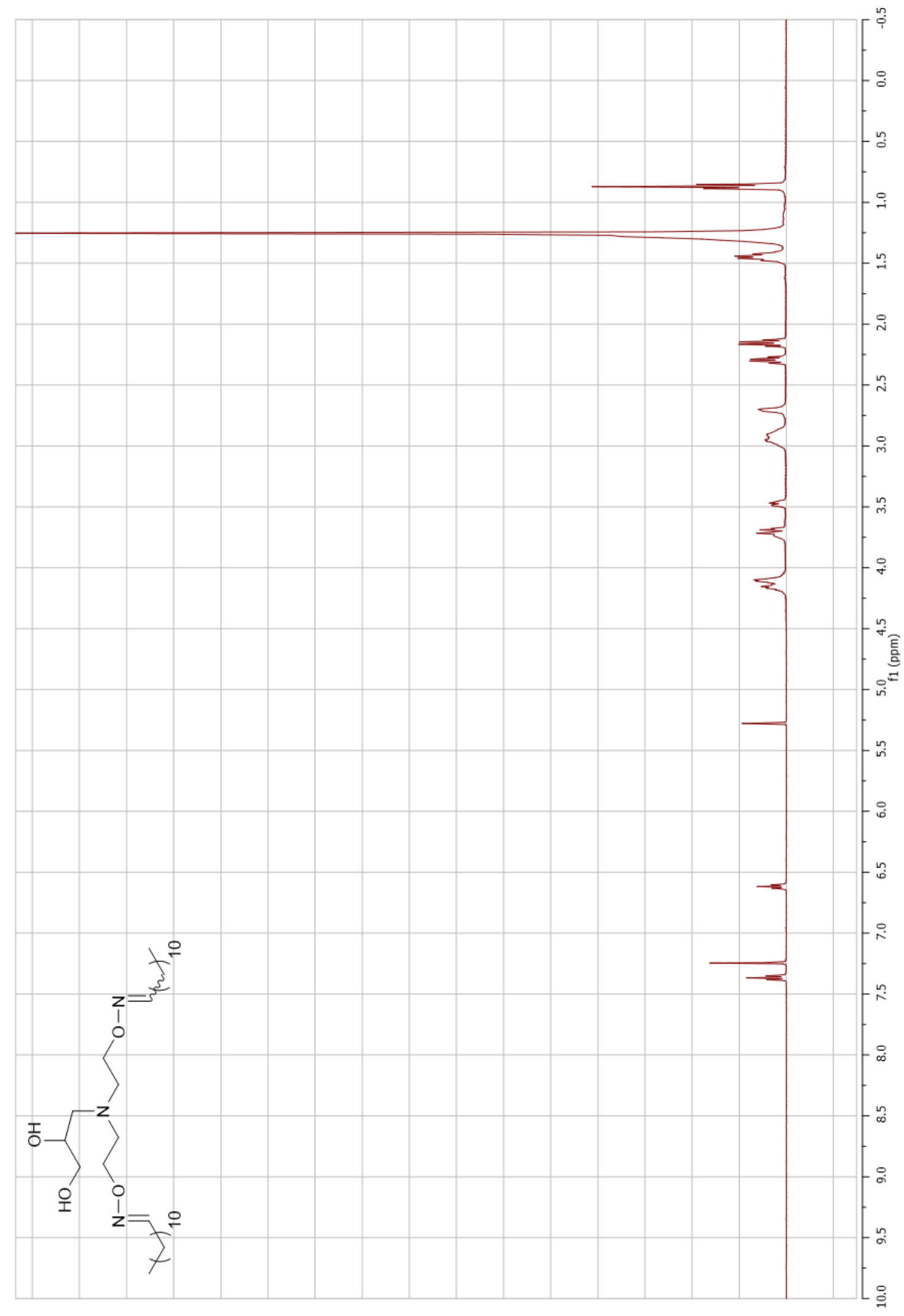

${ }^{1} \mathrm{H}$ NMR of $4.5400 \mathrm{MHz} \mathrm{CDCl}_{3}$ 


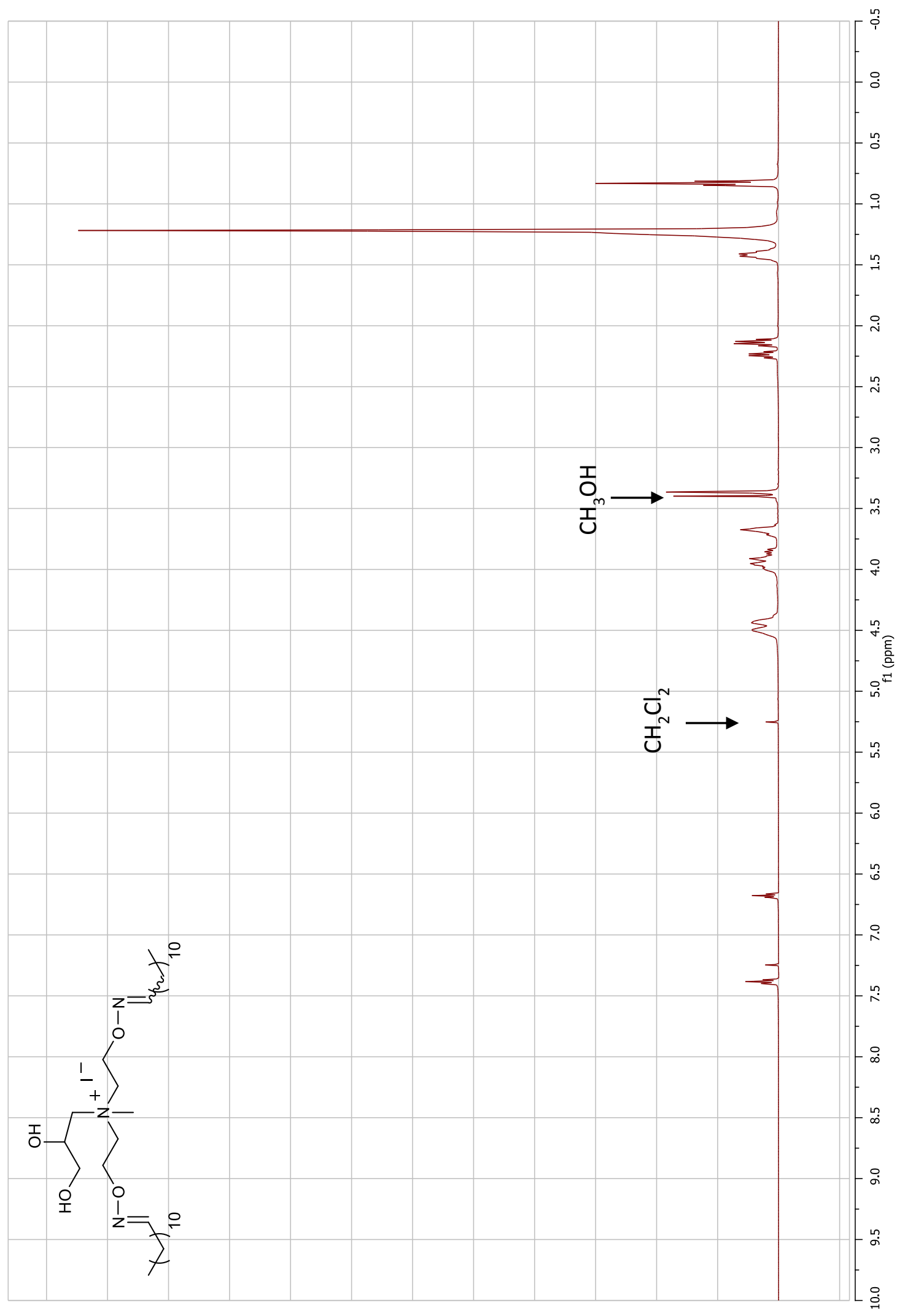

${ }^{1} \mathrm{H}$ NMR of Lipid $1400 \mathrm{MHz} \mathrm{CDCl}_{3}$ 


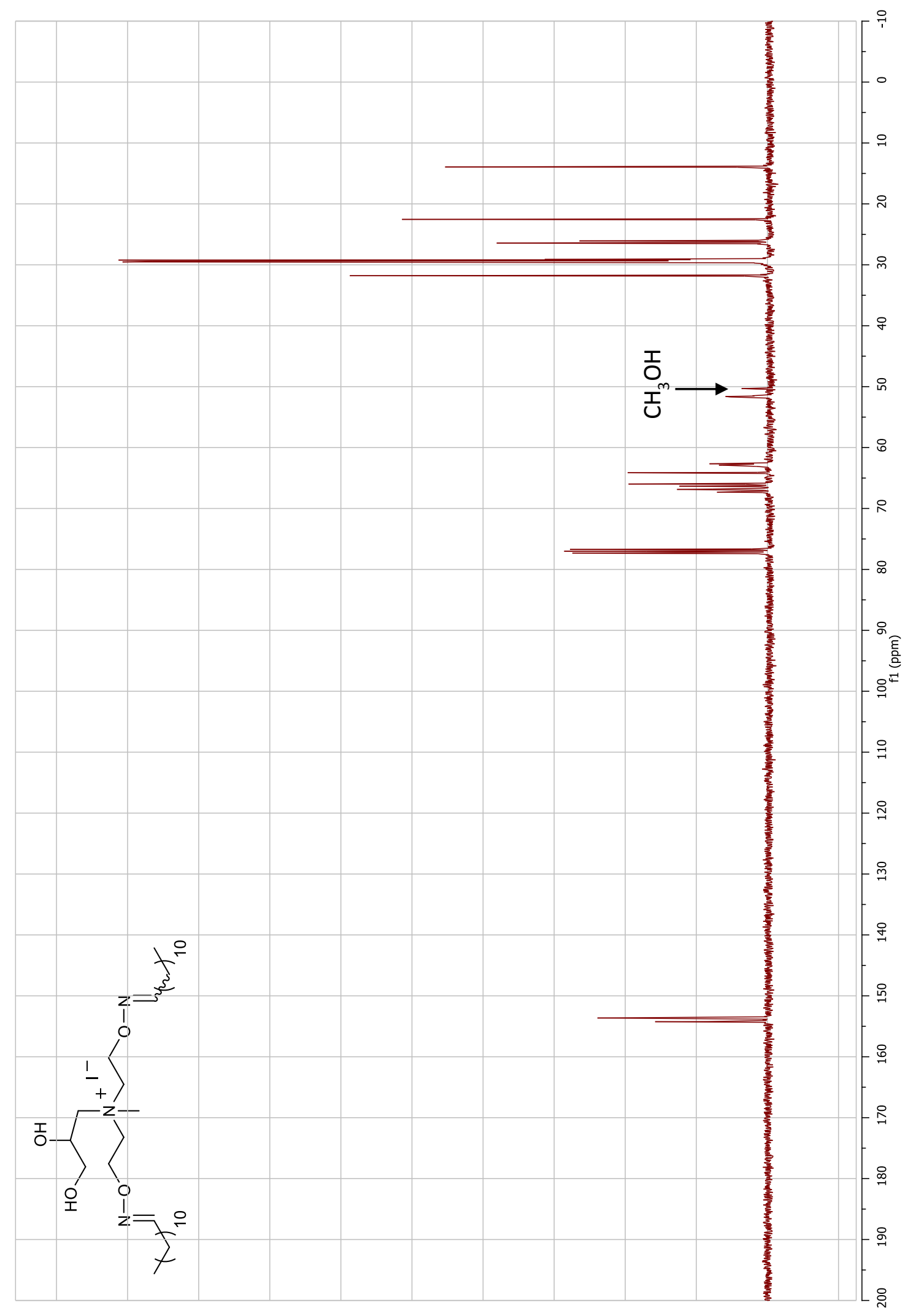

${ }^{13} \mathrm{C}$ NMR of Lipid $1100 \mathrm{MHz} \mathrm{CDCl}_{3}$ 


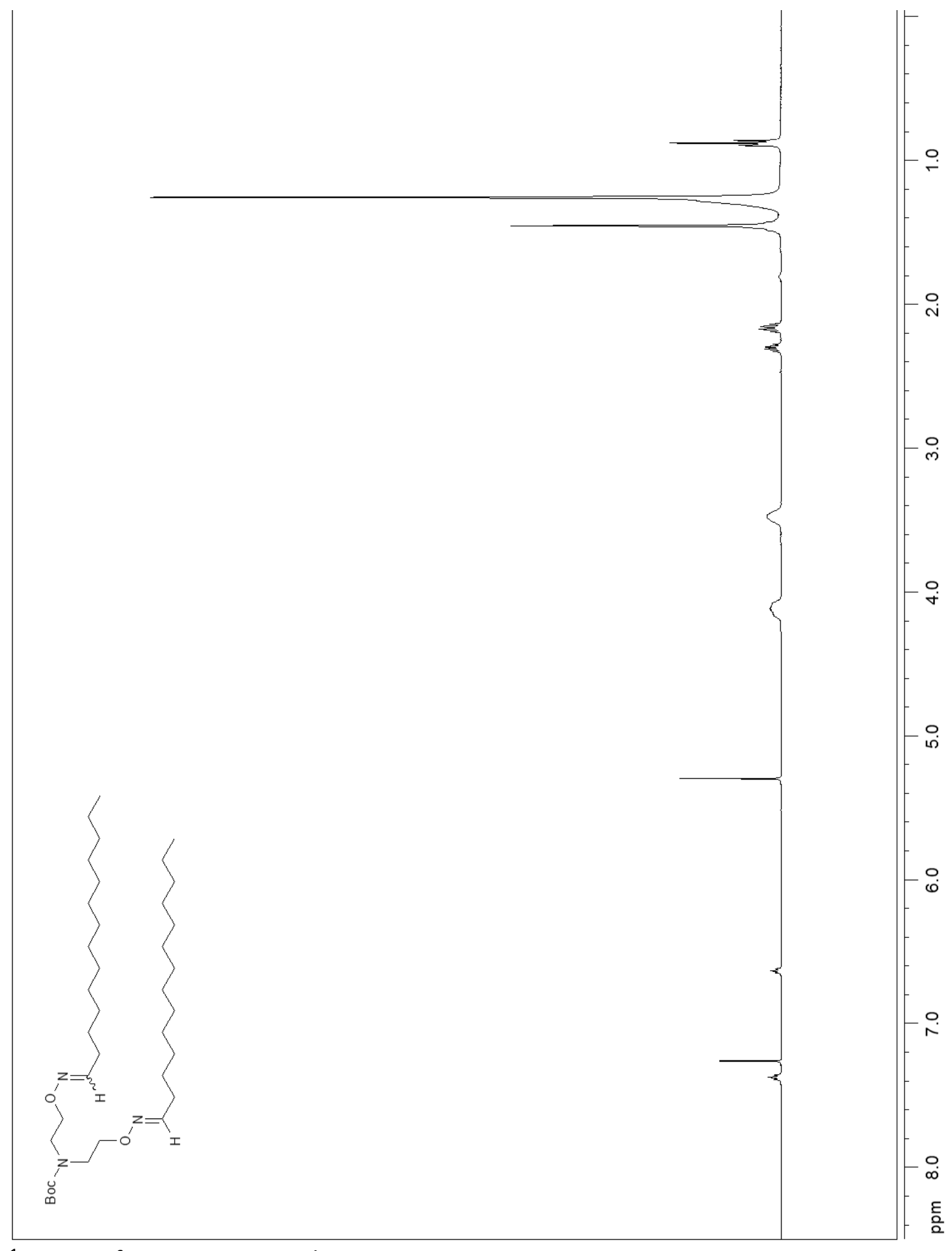

${ }^{1} \mathrm{H}$ NMR of $4.3400 \mathrm{MHz} \mathrm{CDCl}_{3}$ 


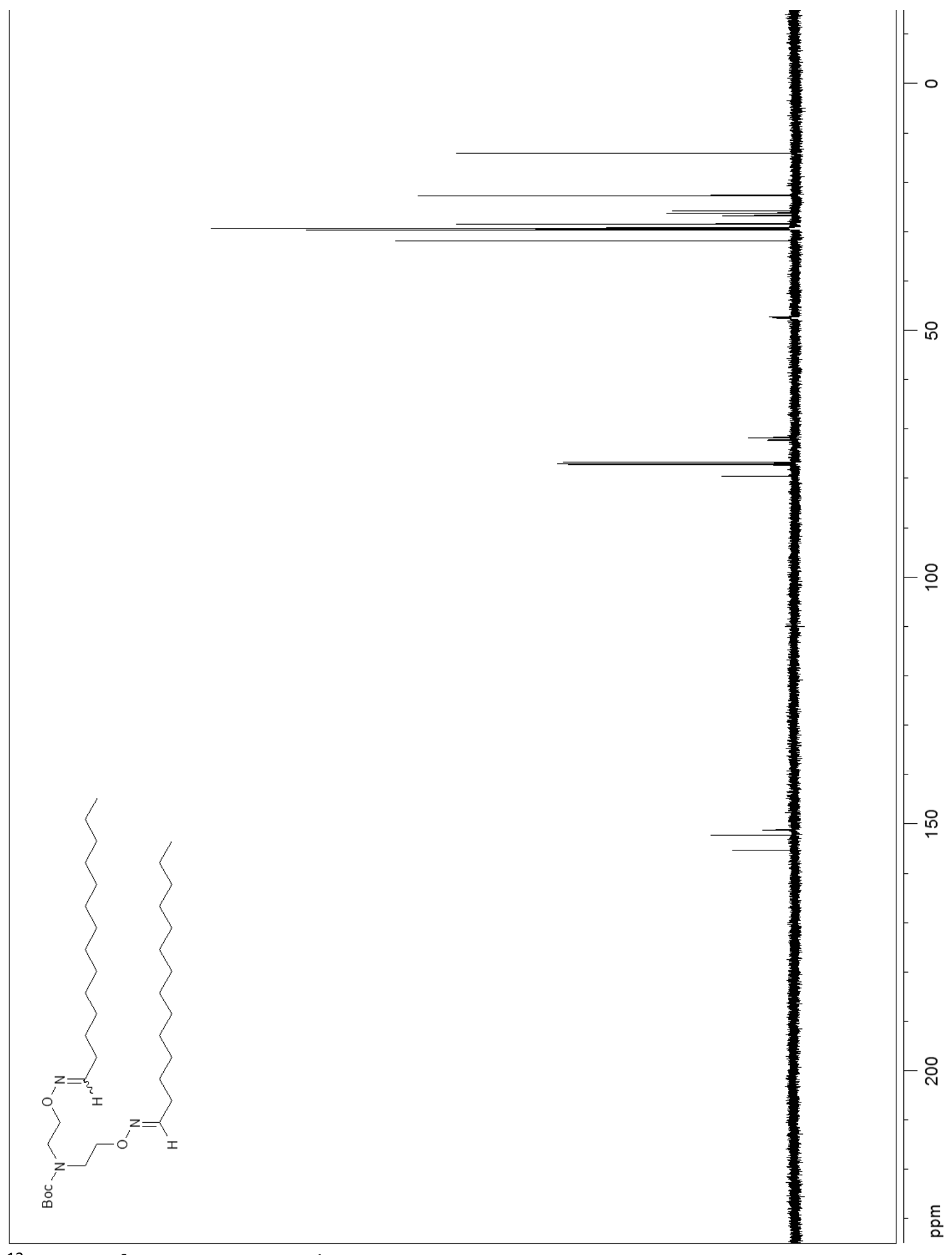

${ }^{13} \mathrm{C}$ NMR of $4.3125 \mathrm{MHz} C D C l_{3}$ 


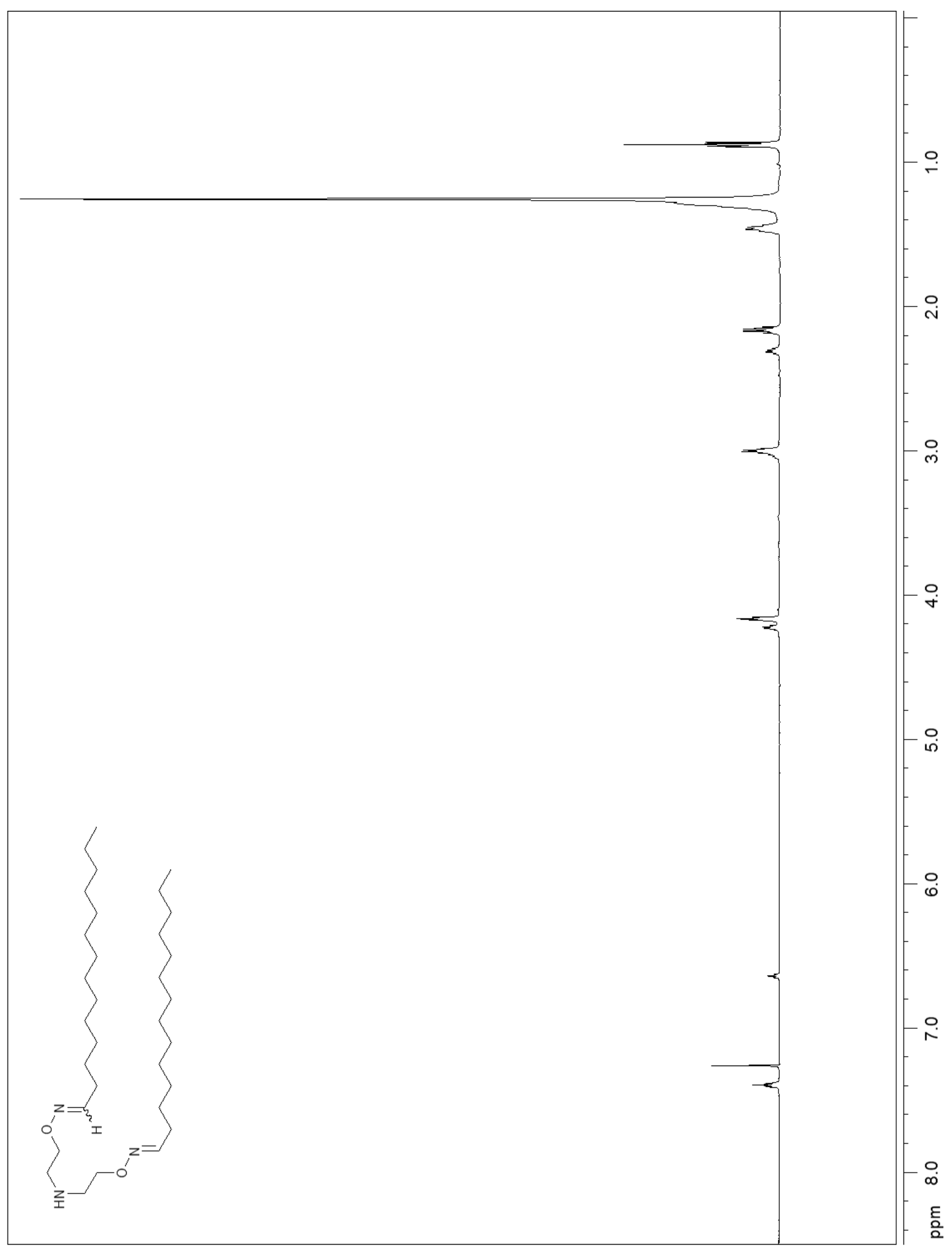

${ }^{1} \mathrm{H} \mathrm{NMR} 500 \mathrm{MHz}^{\mathrm{CDCl}_{3}}$ 


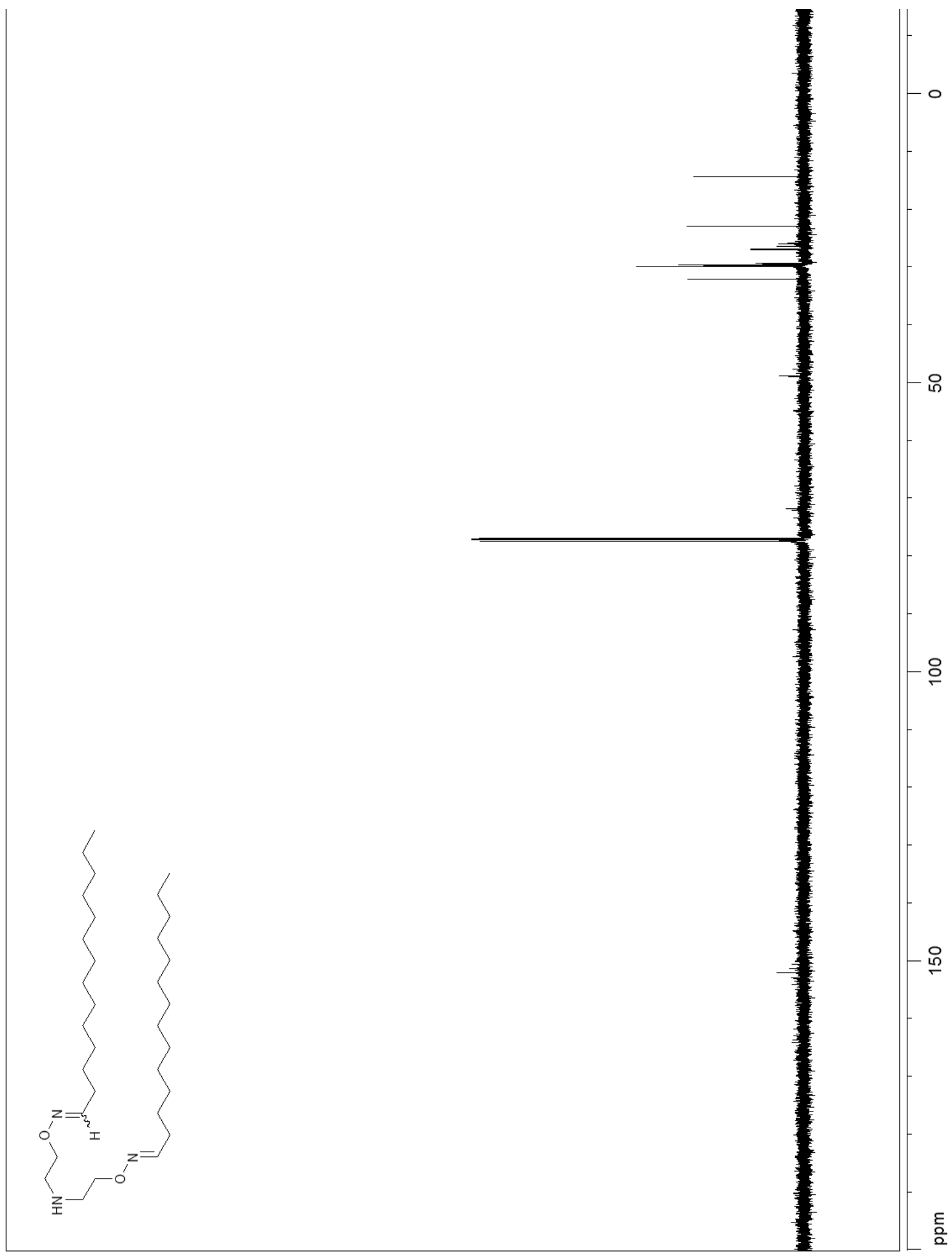

${ }^{13} \mathrm{C}$ NMR $125 \mathrm{MHz}^{\mathrm{CDCl}} 3$ 


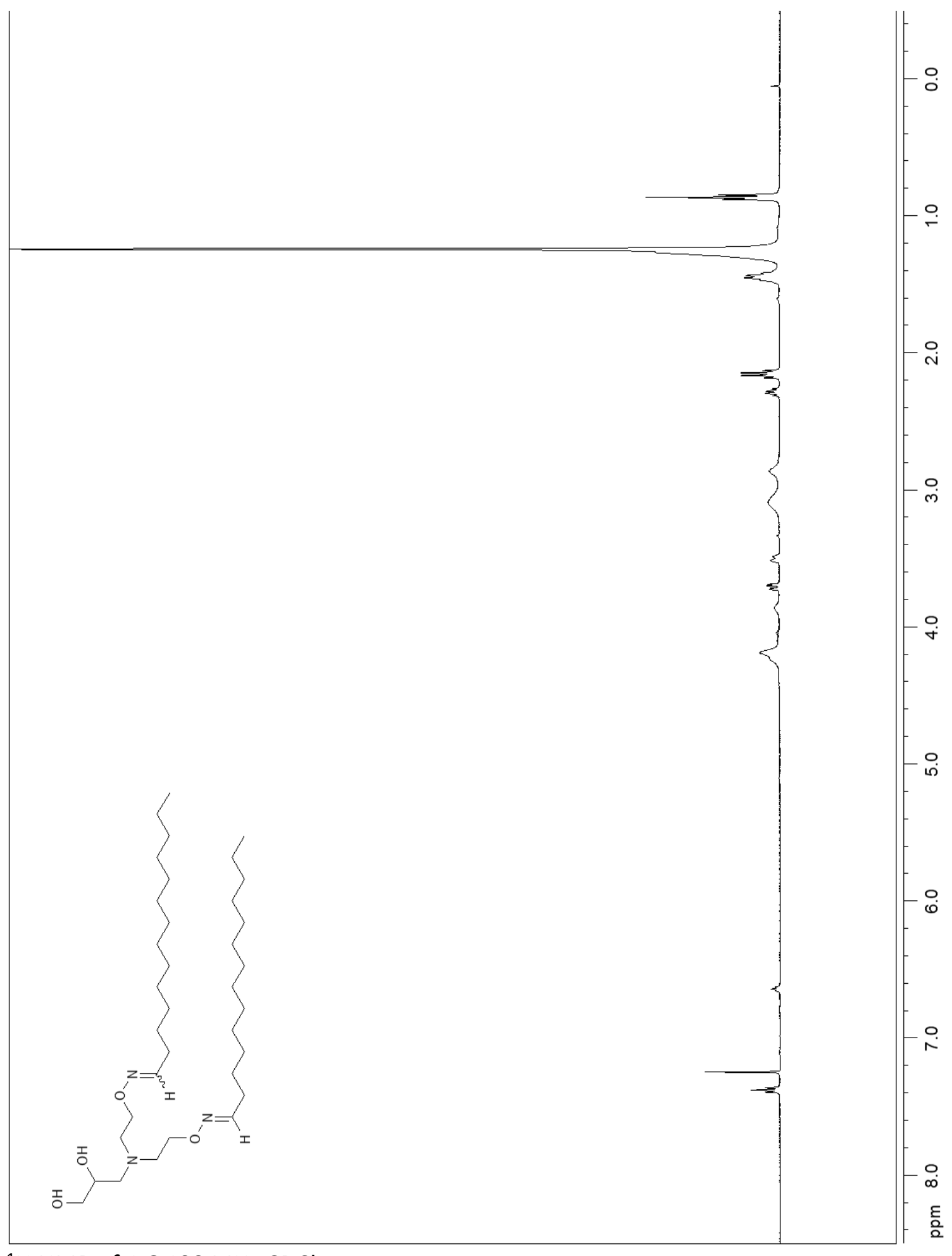

${ }^{1} \mathrm{H} \mathrm{NMR}$ of $4.6400 \mathrm{MHz}^{\mathrm{CDCl}} 3$ 


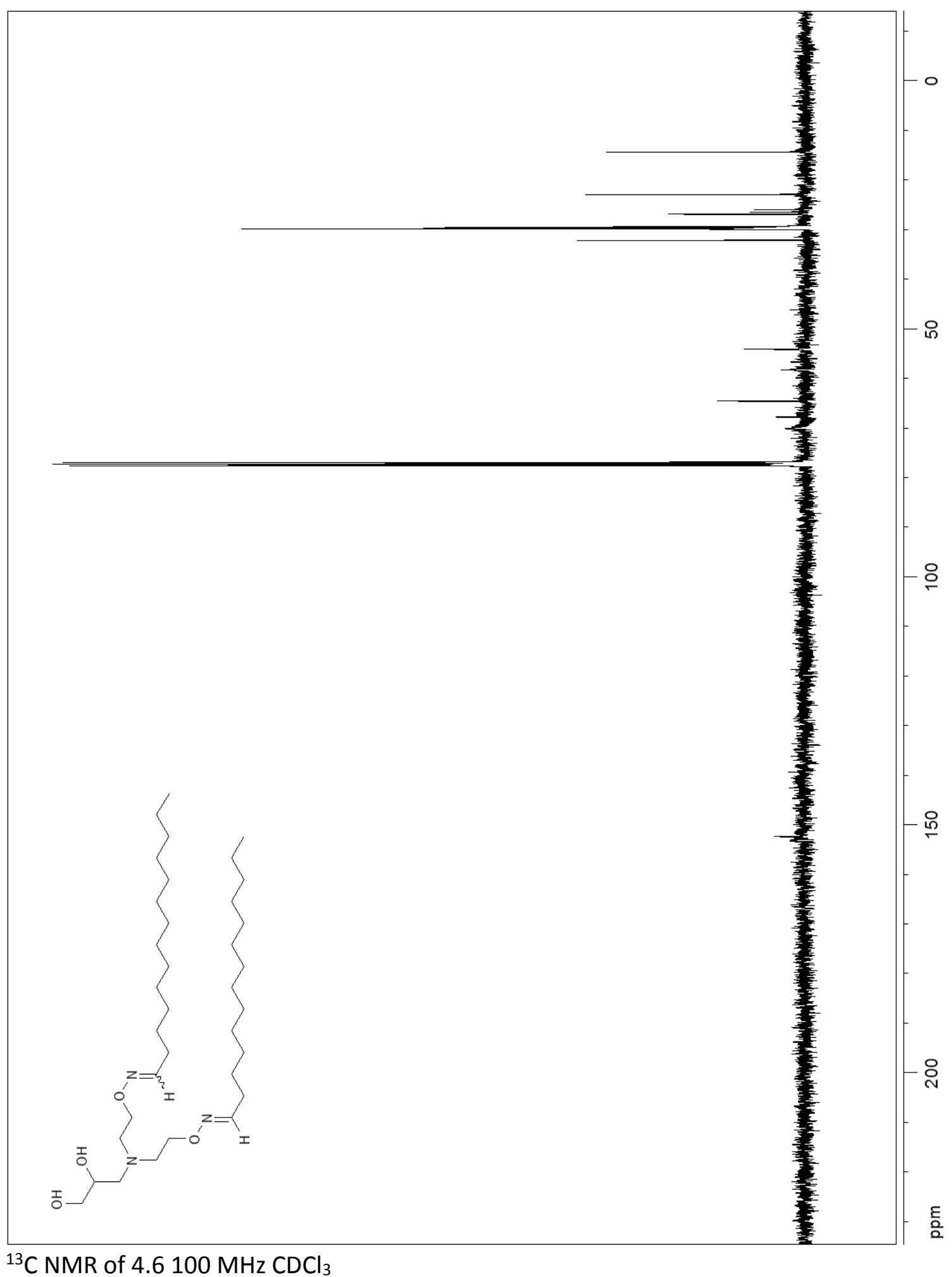




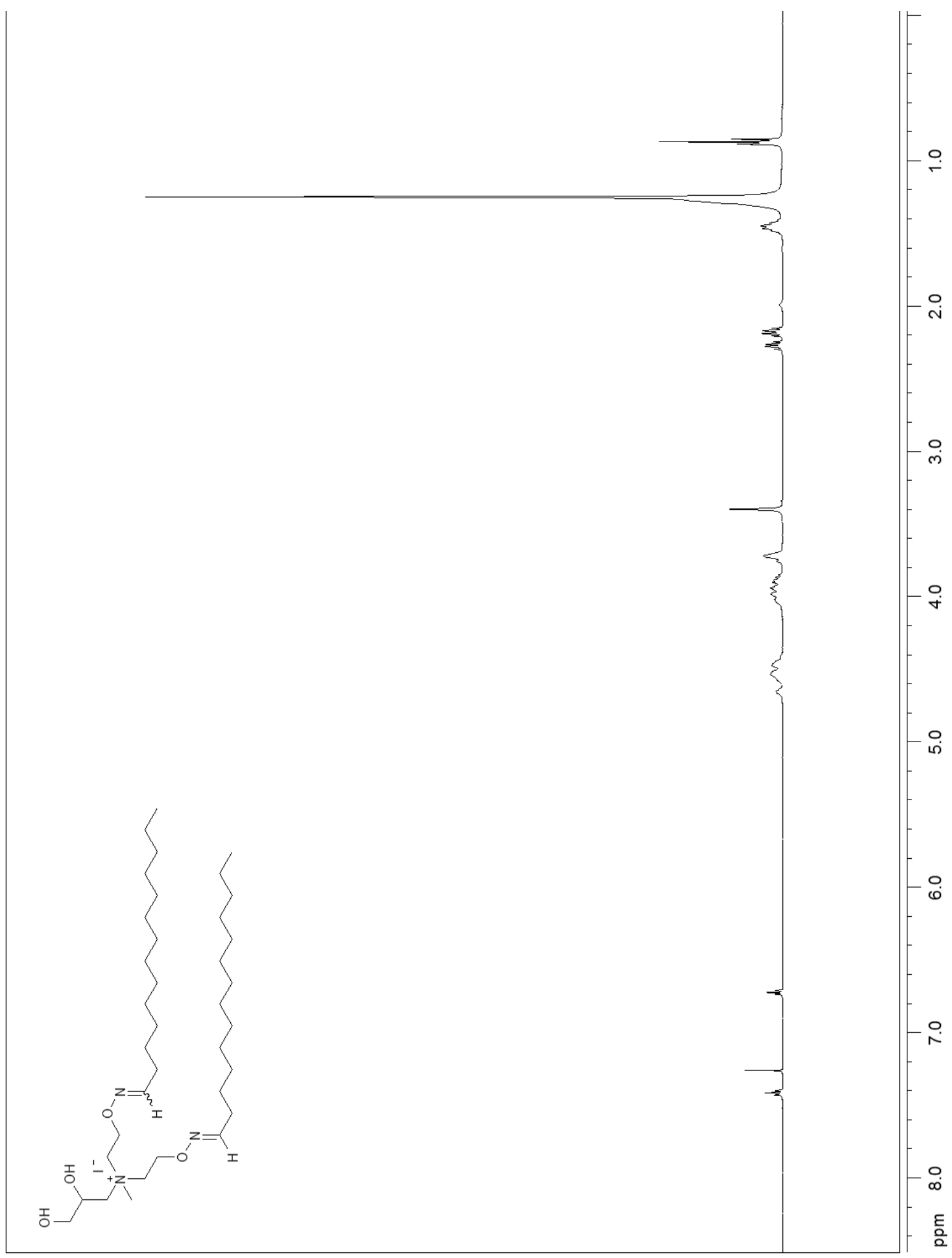

${ }^{1} \mathrm{H}$ NMR of Lipid $2400 \mathrm{MHz} \mathrm{CDCl}_{3}$ 


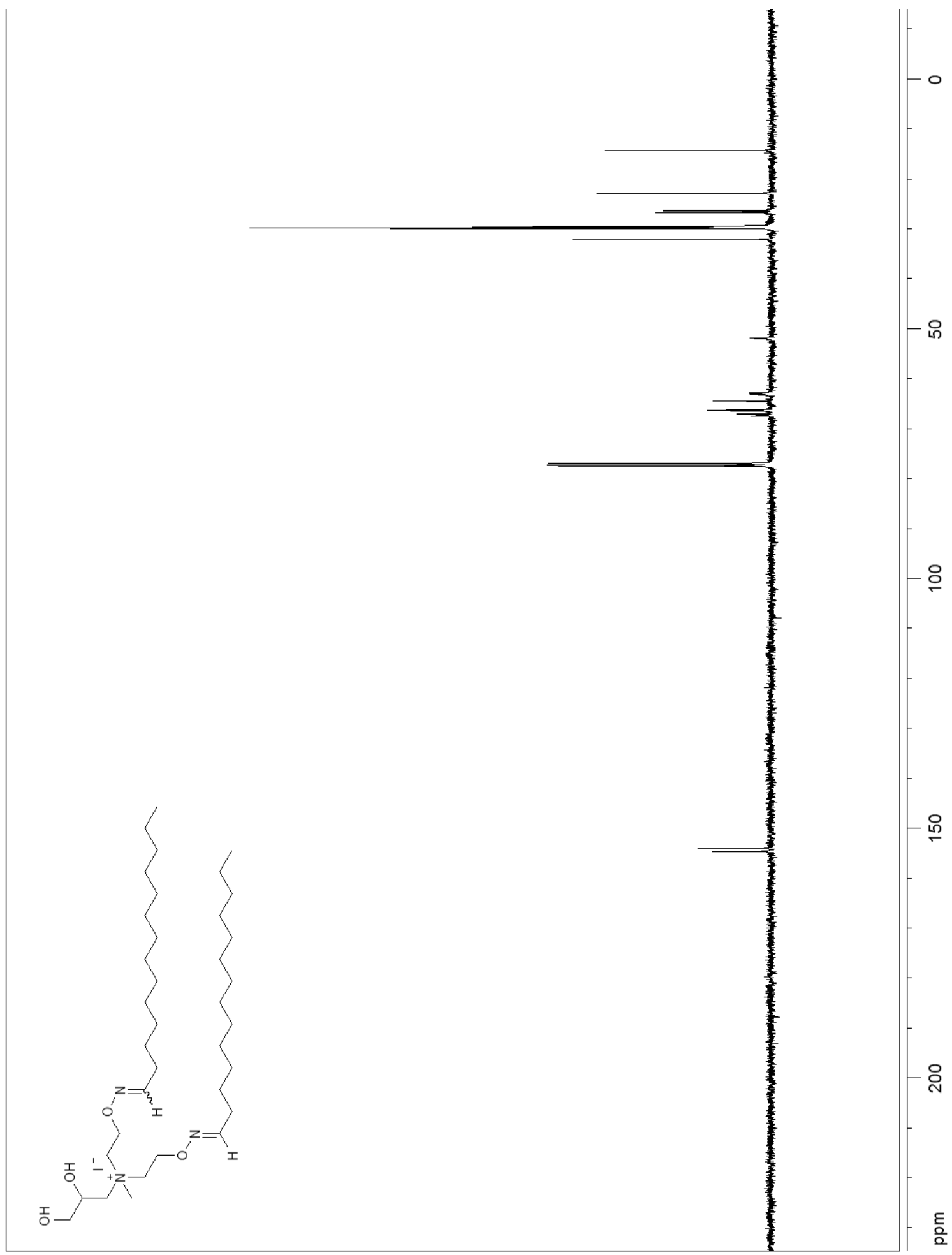

${ }^{13} \mathrm{C}$ NMR of Lipid $2100 \mathrm{MHz} C D C l_{3}$ 


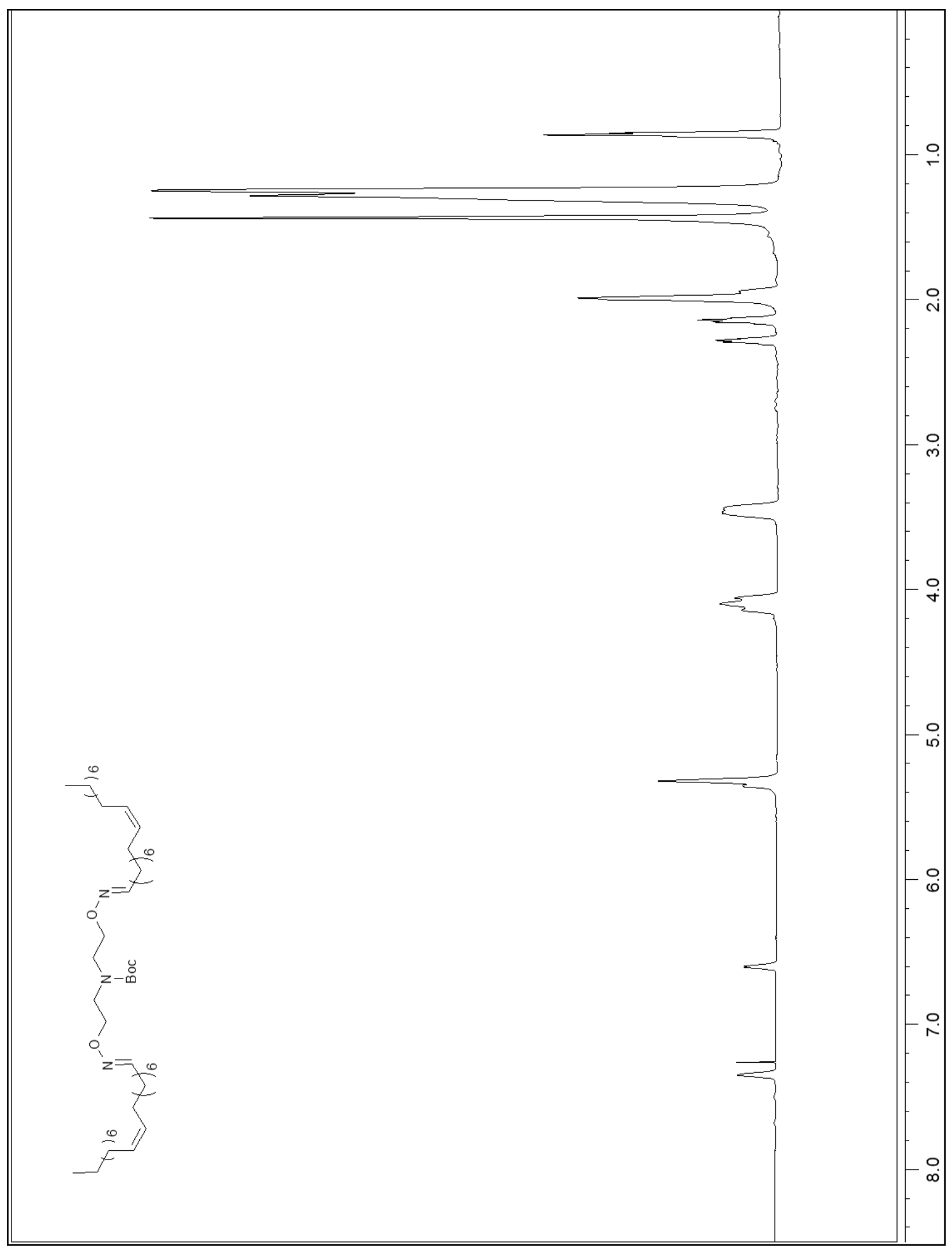

${ }^{1} \mathrm{H}$ NMR of $4.4500 \mathrm{MHz} \mathrm{CDCl}_{3}$ 


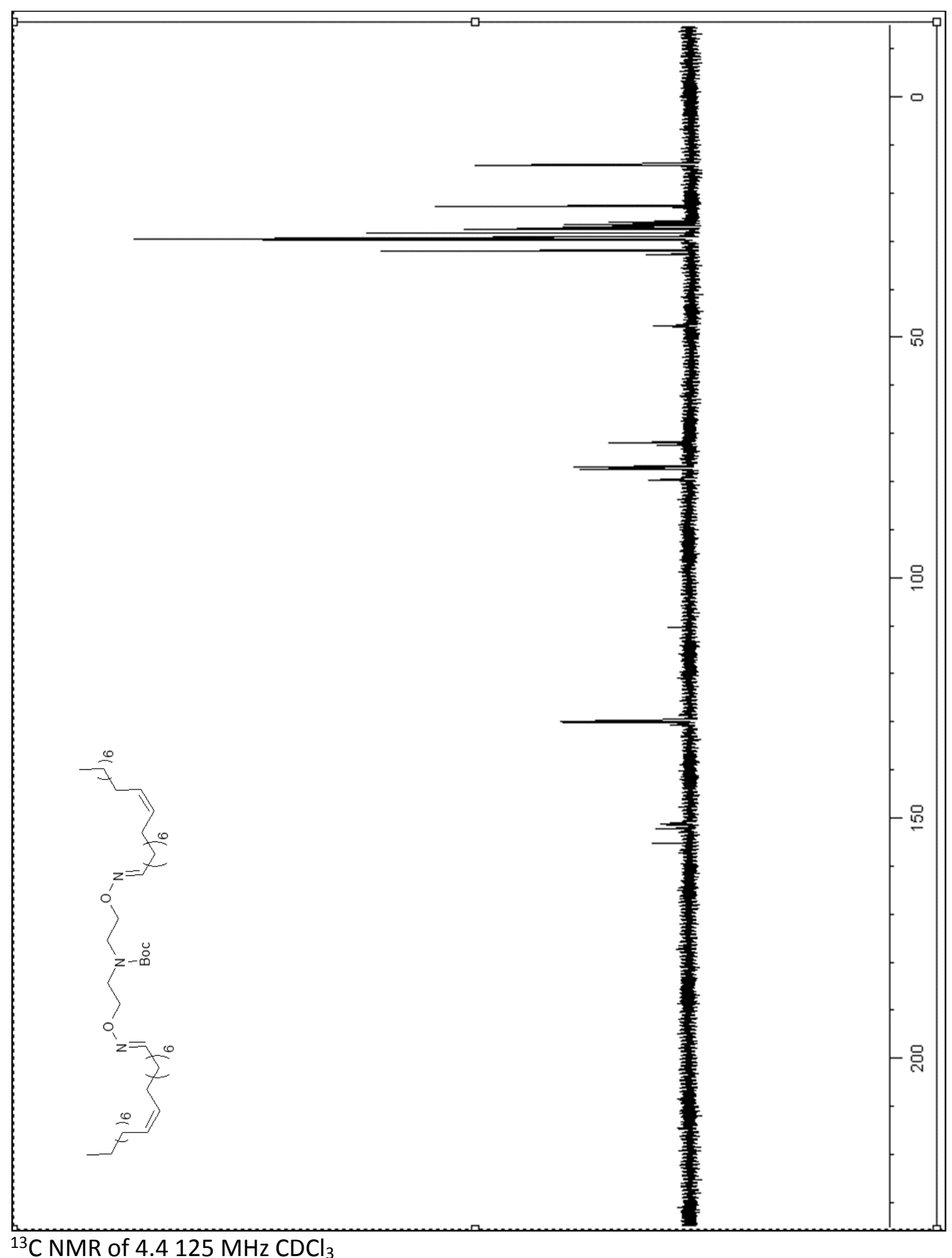




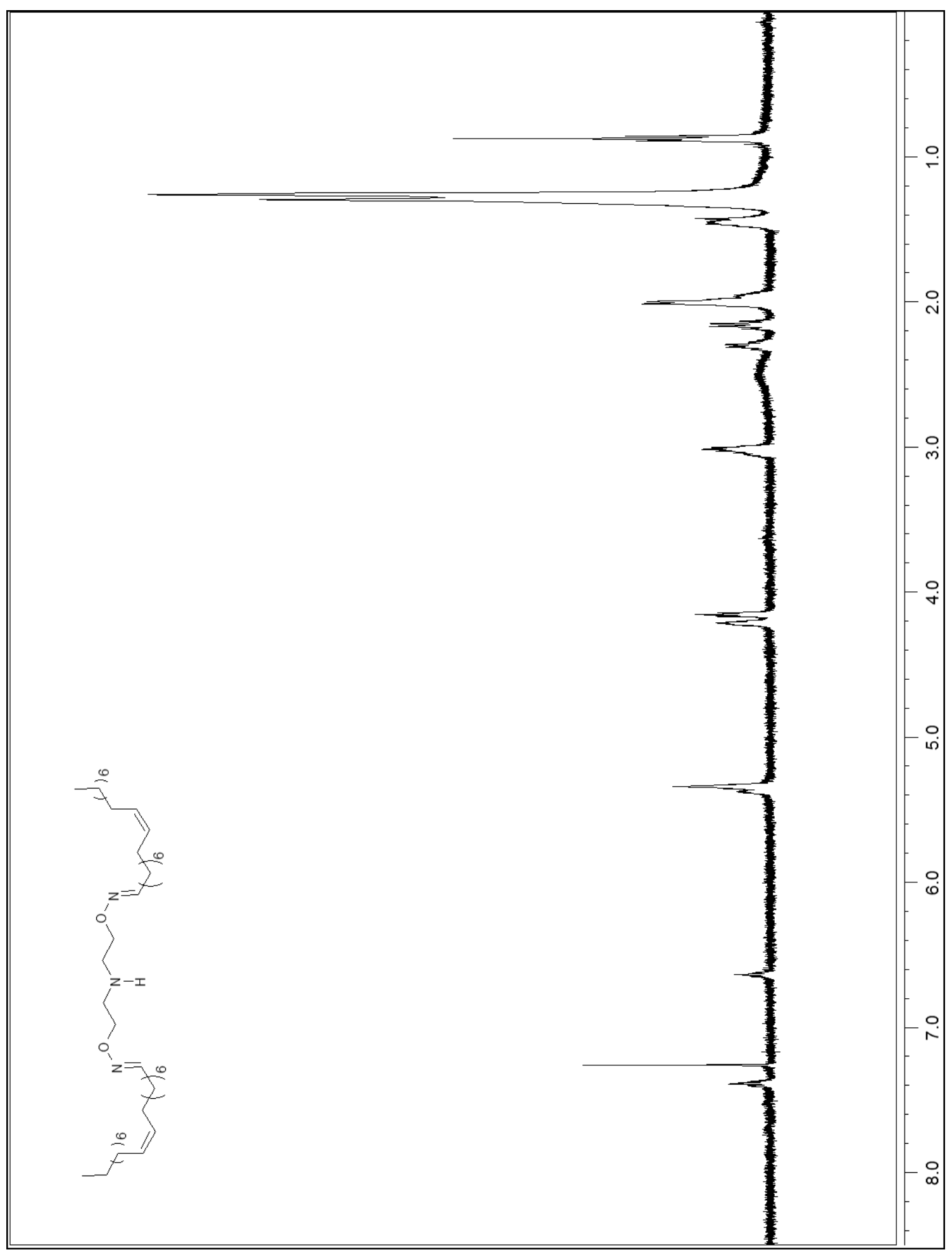

${ }^{1} \mathrm{H}$ NMR $400 \mathrm{MHz} \mathrm{CDCl}_{3}$ 


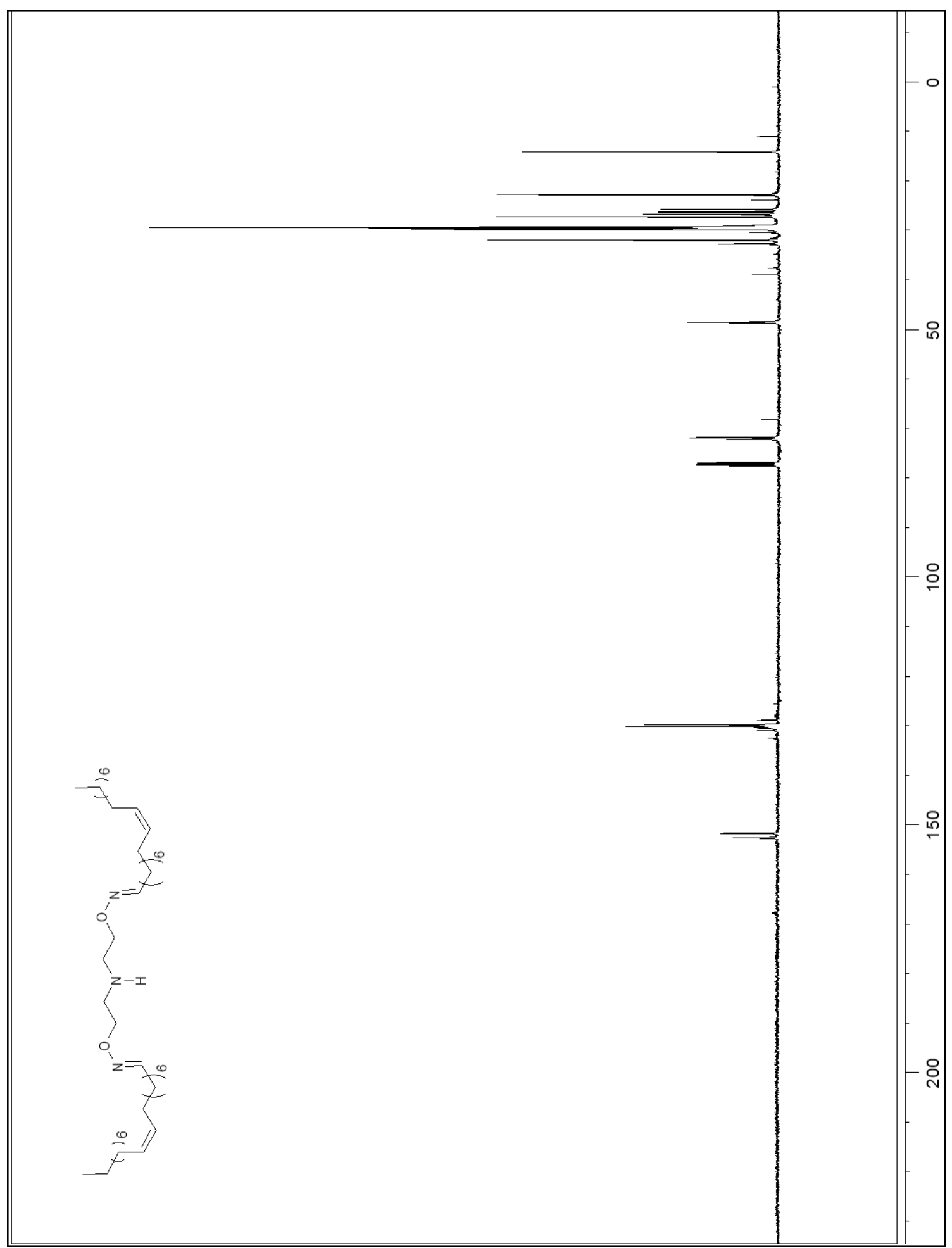

${ }^{13} \mathrm{C} \mathrm{NMR} 125 \mathrm{MHz}^{\mathrm{CDCl}} 3$ 


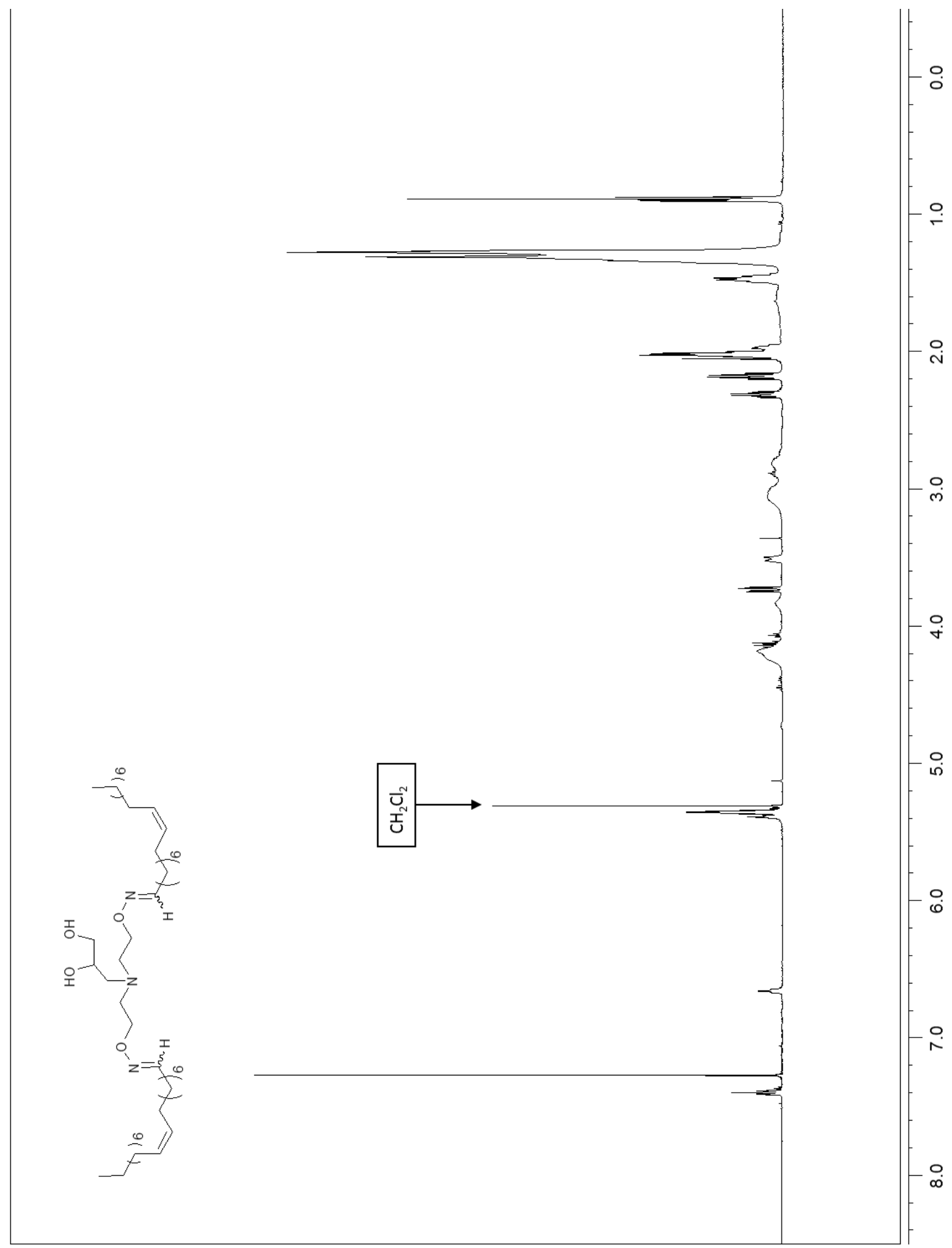

${ }^{1} \mathrm{H} \mathrm{NMR}$ of $4.7500 \mathrm{MHz}^{\mathrm{CDCl}} 3$ 


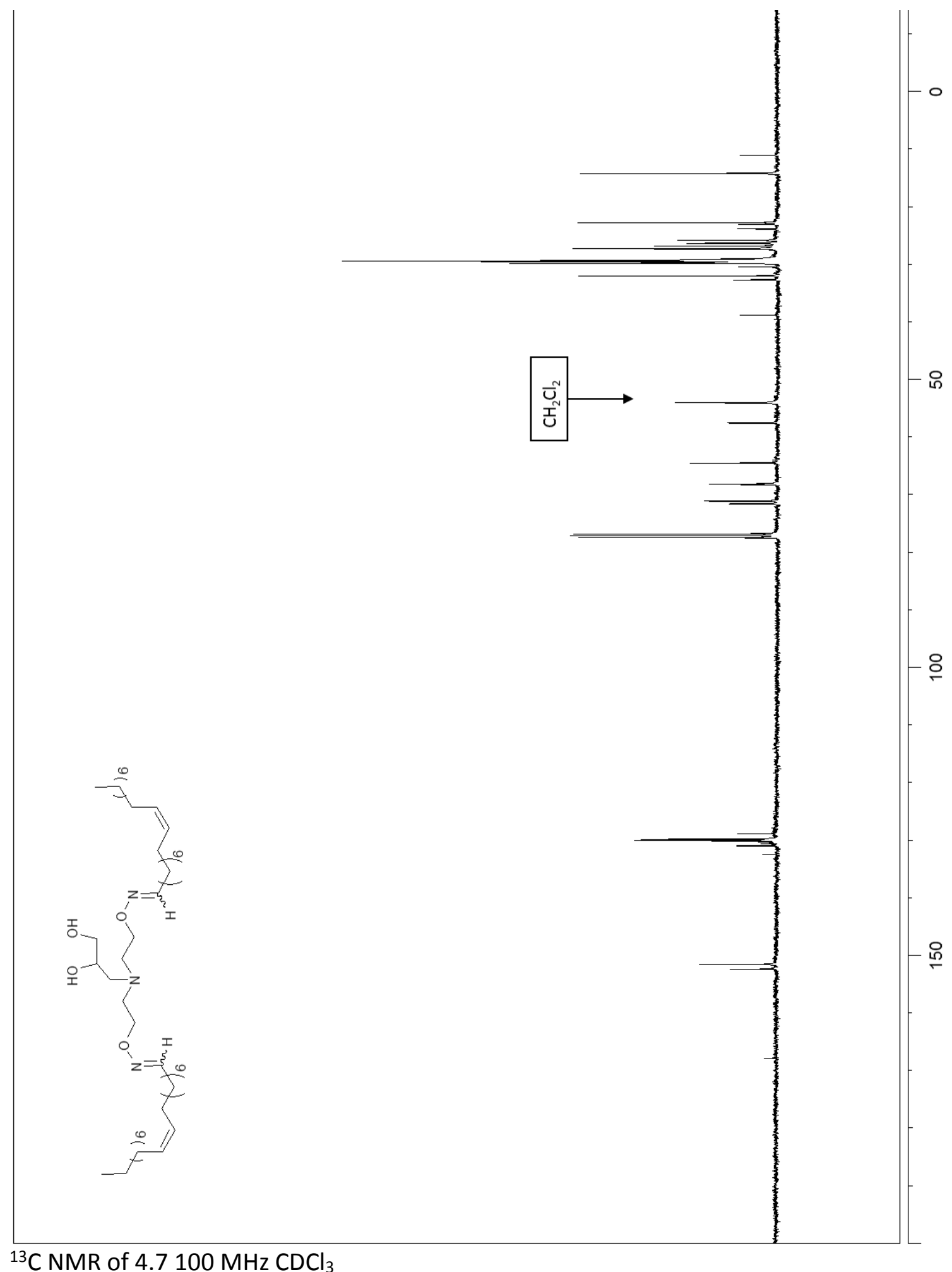




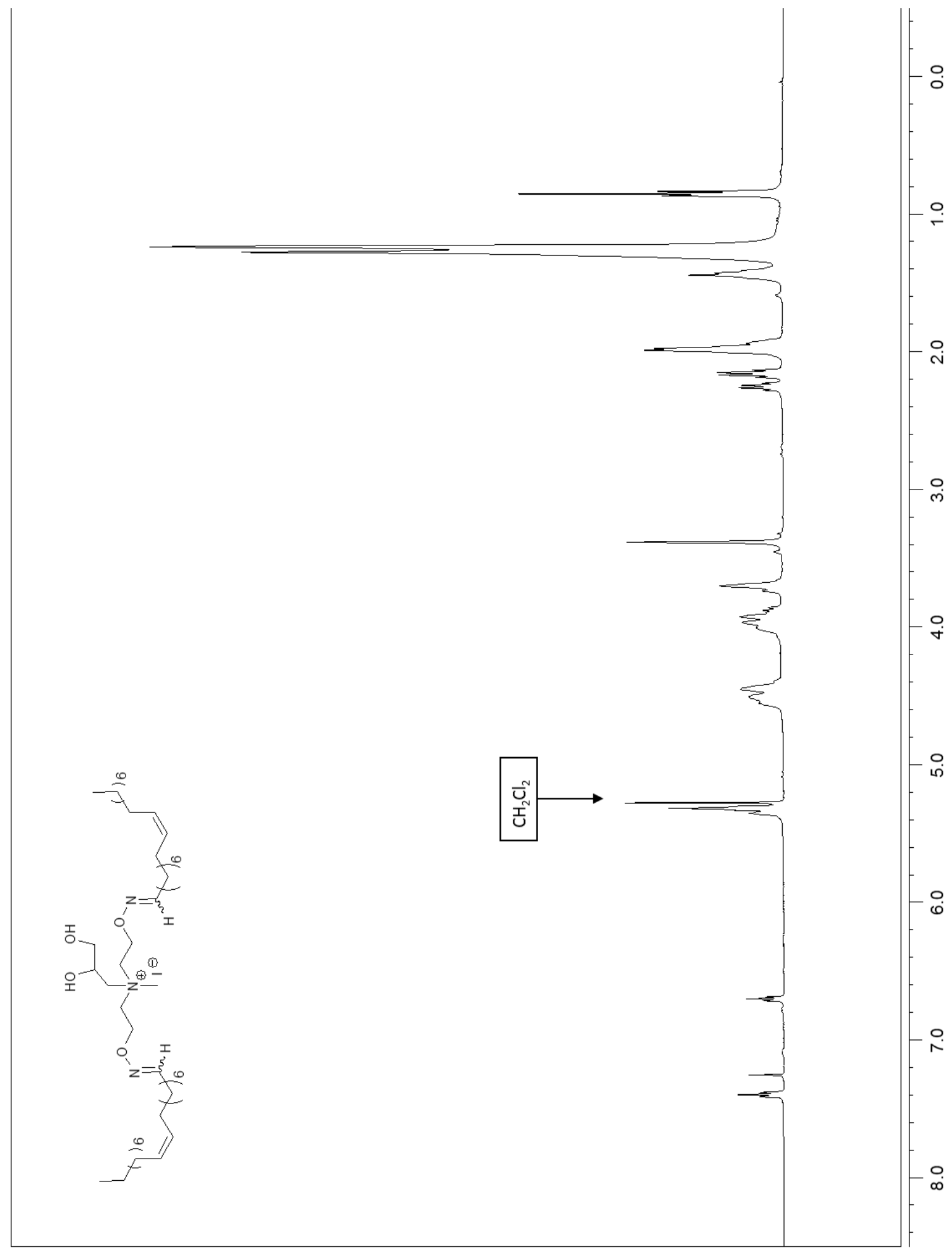

${ }^{1} \mathrm{H}$ NMR of Lipid $3400 \mathrm{MHz} \mathrm{CDCl}_{3}$ 


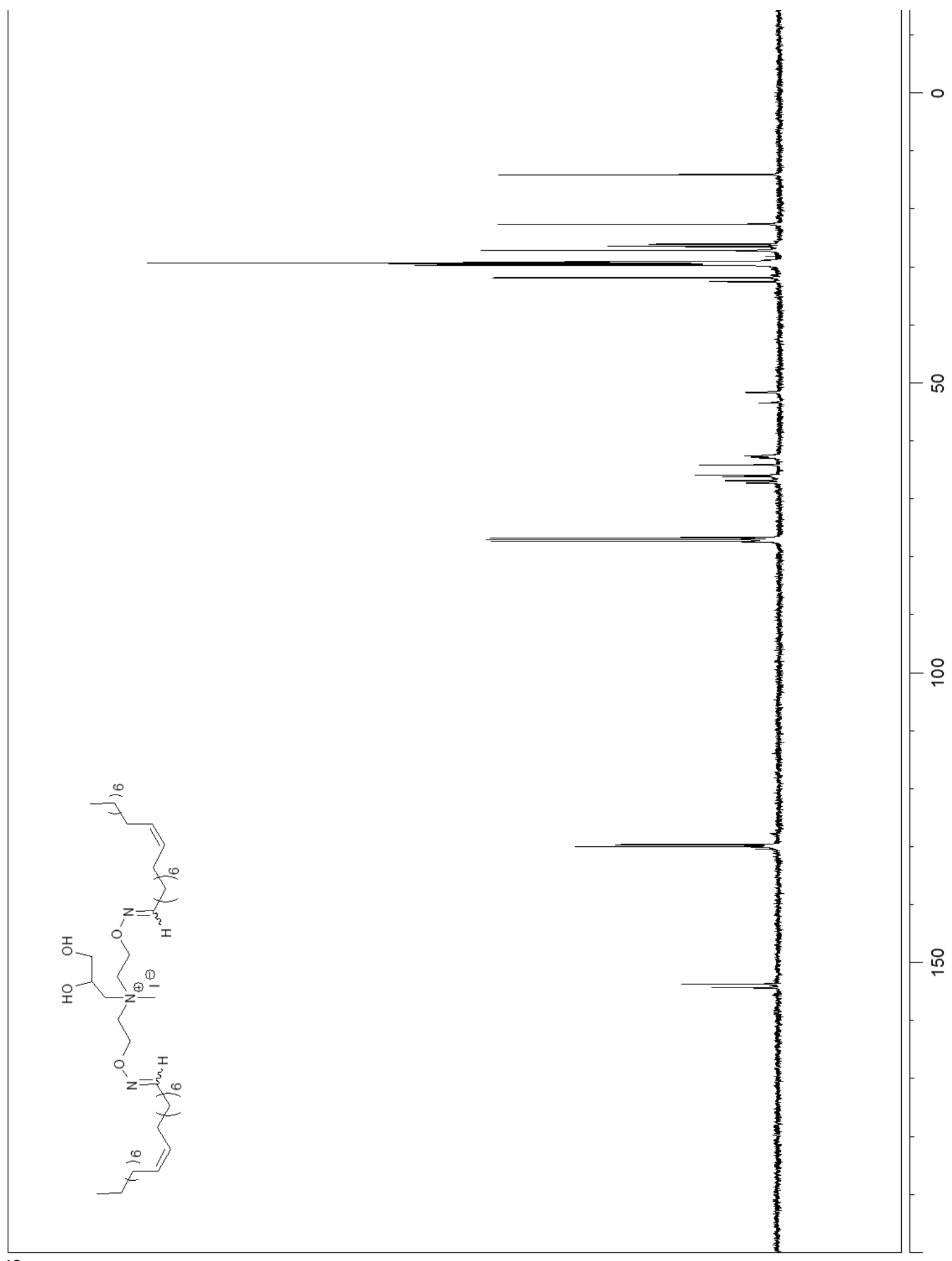

${ }^{13} \mathrm{C}$ NMR of Lipid $3100 \mathrm{MHz} \mathrm{CDCl}_{3}$ 


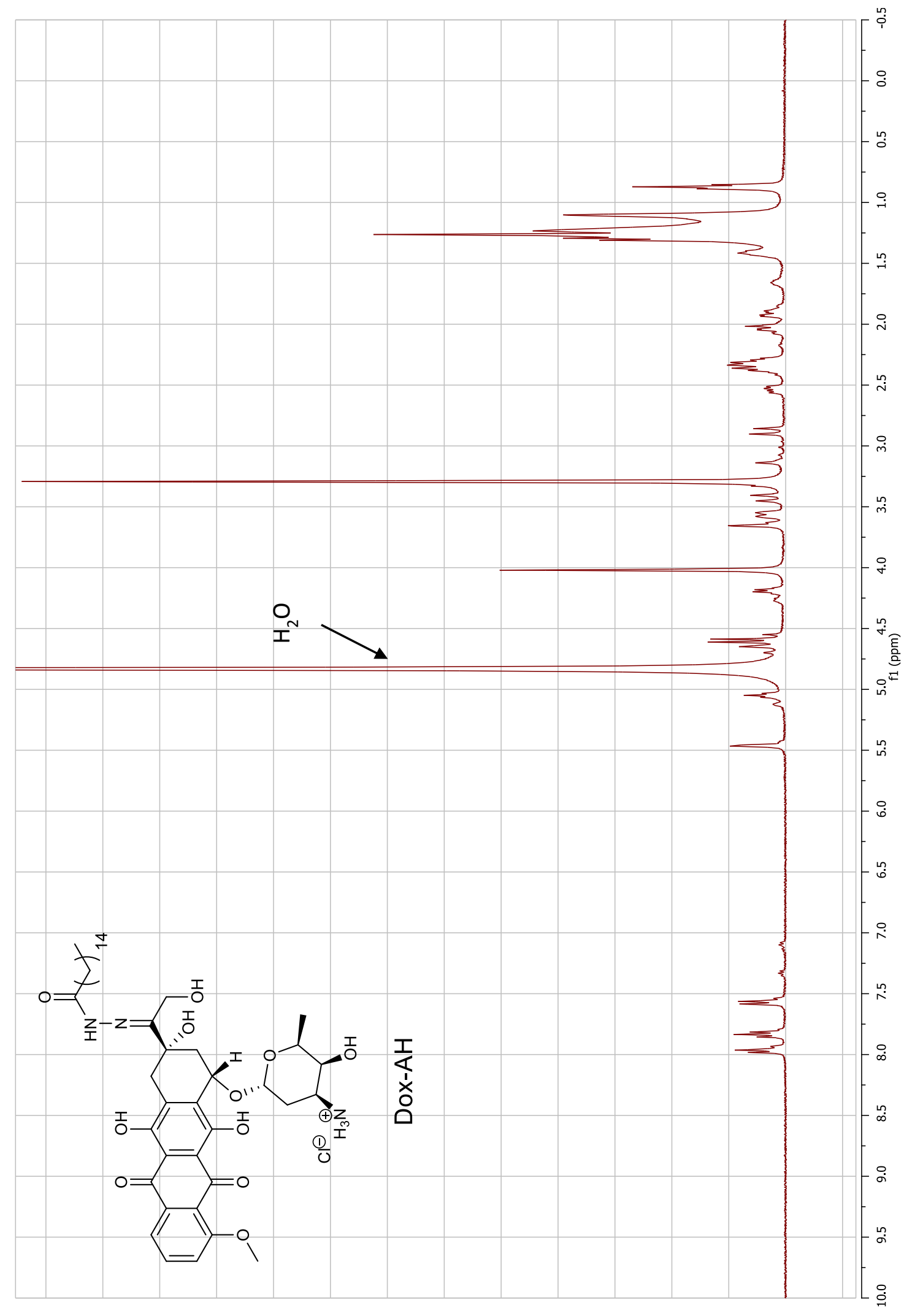

${ }^{1} \mathrm{H}$ NMR of Dox-AH $400 \mathrm{MHz} \mathrm{CD}_{3} \mathrm{OD}$ 


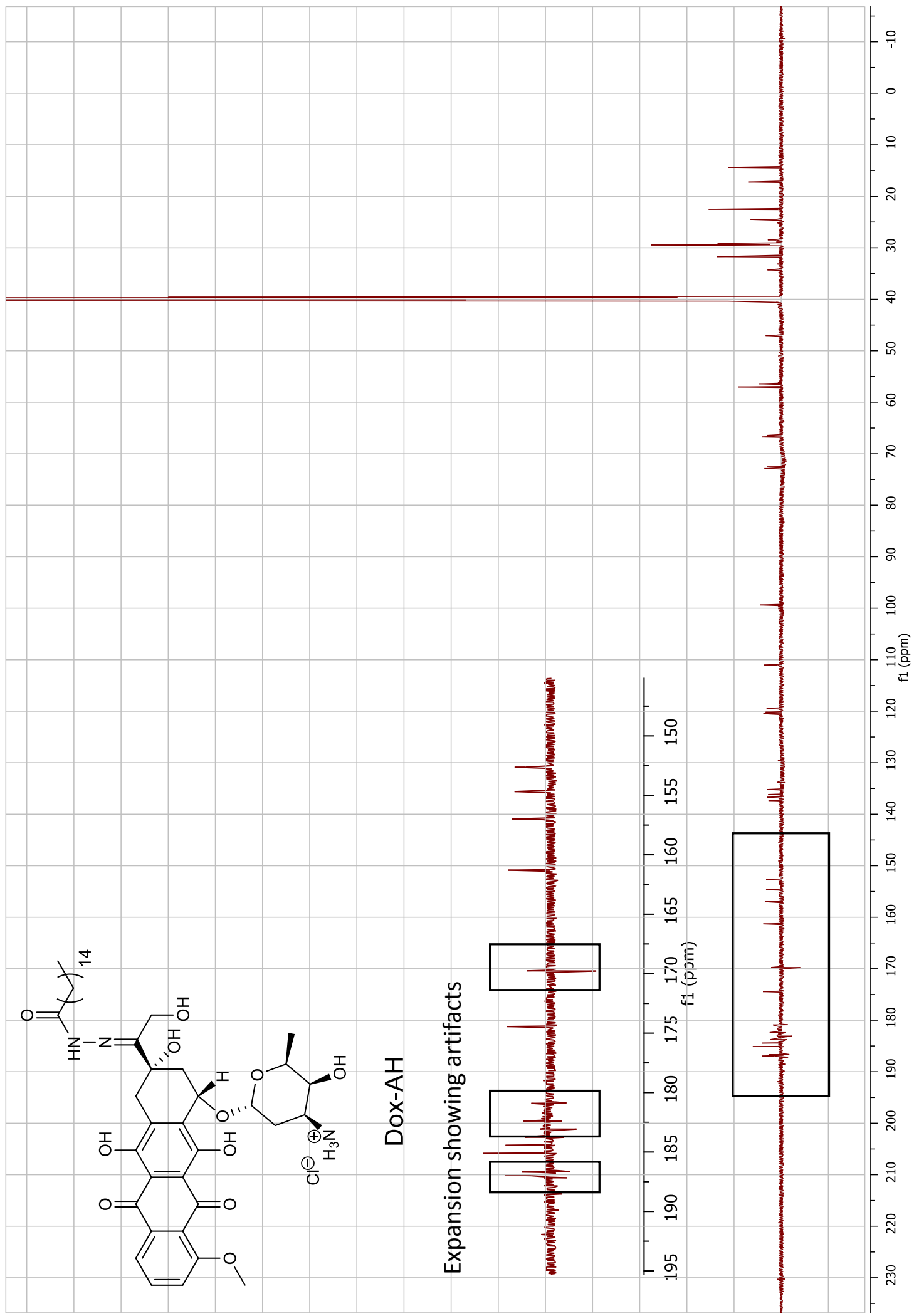

${ }^{13} \mathrm{C}$ NMR of Dox-AH $100 \mathrm{MHz} \mathrm{CD}_{3} \mathrm{OD}$ 


\section{A.2. ZETA-POTENTIAL MEASUREMENTS}

\section{Zeta-potential for NPs based on work up}

\begin{tabular}{|c|c|c|c|c|c|}
\hline \multirow{10}{*}{$\begin{array}{r}\text { Run } \\
1 \\
2 \\
3 \\
4 \\
5 \\
6 \\
7 \\
8 \\
9 \\
10 \\
\end{array}$} & \multirow{10}{*}{$\begin{array}{l}\text { Mobility } \\
0.26 \\
-0.25 \\
-0.87 \\
0.48 \\
0.50 \\
0.24 \\
0.33 \\
0.68 \\
0.43 \\
0.18 \\
\end{array}$} & Zeta Potential (mV) & Rel. Residual & \multicolumn{2}{|c|}{ Measurement Parameters: } \\
\hline & & \multirow{9}{*}{$\begin{array}{r}-3.37 \\
-3.26 \\
-11.14 \\
6.18 \\
-6.37 \\
-3.05 \\
4.24 \\
8.65 \\
-5.53 \\
2.27 \\
\end{array}$} & \multirow{9}{*}{$\begin{array}{l}0.0415 \\
0.0533 \\
0.0883 \\
0.0460 \\
0.0335 \\
0.0415 \\
0.0446 \\
0.0868 \\
0.0438 \\
0.0473 \\
\end{array}$} & Conductance & $=30 \mu \mathrm{S}$ \\
\hline & & & & Electric Field & $=10.14 \mathrm{~V} / \mathrm{cm}$ \\
\hline & & & & Sample Count Rate & $=466 \mathrm{kcps}$ \\
\hline & & & & Ref. Count Rate & $=1121 \mathrm{kcps}$ \\
\hline & & & & & \\
\hline & & & & & \\
\hline & & & & & \\
\hline & & & & & \\
\hline & & & & & \\
\hline Mean & .0 .09 & $\cdot 1.14$ & 0.0526 & & \\
\hline Std. Error & 0.15 & 1.97 & 0.0060 & & \\
\hline Combined & .0 .14 & -1.84 & 0.0224 & & \\
\hline
\end{tabular}

\section{Rinsed NPs}

\begin{tabular}{|c|c|c|c|c|c|}
\hline \multirow{10}{*}{$\begin{array}{r}\text { Run } \\
1 \\
2 \\
3 \\
4 \\
5 \\
6 \\
7 \\
8 \\
9 \\
10 \\
\end{array}$} & \multirow{10}{*}{$\begin{array}{l}\text { Mobility } \\
-1.40 \\
-1.41 \\
-2.70 \\
-1.61 \\
-1.53 \\
-1.42 \\
-1.19 \\
-1.23 \\
-1.52 \\
-1.89 \\
\end{array}$} & \multirow{10}{*}{$\begin{array}{l}\text { Zeta Potential (mV) } \\
-17.86 \\
-17.98 \\
-34.61 \\
-20.64 \\
-19.61 \\
-18.13 \\
-15.17 \\
-15.73 \\
-19.47 \\
.24 .14 \\
\end{array}$} & \multirow{10}{*}{$\begin{array}{l}\text { Rel. Residual } \\
0.0891 \\
0.0816 \\
0.2664 \\
0.0695 \\
0.0827 \\
0.0861 \\
0.0628 \\
0.0495 \\
0.0640 \\
0.0777 \\
\end{array}$} & \multicolumn{2}{|c|}{ | Measurement Parameters: } \\
\hline & & & & \multirow{12}{*}{\begin{tabular}{|l|} 
Conductance \\
Electric Field \\
Sample Count Rate \\
Ref. Count Rate
\end{tabular}} & $=27 \mu \mathrm{S}$ \\
\hline & & & & & $=10.07 \mathrm{~V} / \mathrm{cm}$ \\
\hline & & & & & $=213 \mathrm{kcps}$ \\
\hline & & & & & $=1033 \mathrm{kcps}$ \\
\hline & & & & & \\
\hline & & & & & \\
\hline & & & & & \\
\hline & & & & & \\
\hline & & & & & \\
\hline Mean & .1 .59 & .20 .33 & 0.0929 & & \\
\hline Std. Error & 0.14 & 1.78 & 0.0197 & & \\
\hline Combined & -1.46 & -18.73 & 0.0399 & & \\
\hline
\end{tabular}

Multiply rinsed NPs

\begin{tabular}{|c|c|c|c|c|c|}
\hline Run & Mobility & Zeta Potential (mV) & Rel. Residual & \multicolumn{2}{|c|}{ Measurement Parameters: } \\
\hline 1 & 3.75 & 48.00 & 0.0630 & \begin{tabular}{|l|} 
Conductance \\
\end{tabular} & $=80 \mu \mathrm{S}$ \\
\hline 2 & 4. 09 & 52.28 & 0.0603 & Electric Field & $=9.50 \mathrm{~V} / \mathrm{cm}$ \\
\hline 3 & 3. 83 & 49. 06 & 0.0607 & Sample Count Rate & $=430 \mathrm{kcps}$ \\
\hline $\begin{array}{l}4 \\
5\end{array}$ & $\begin{array}{l}4.76 \\
5.10\end{array}$ & $\begin{array}{l}60.90 \\
65.21\end{array}$ & $\begin{array}{l}0.0831 \\
0.0534\end{array}$ & Ref. Count Rate & $=1055 \mathrm{kcps}$ \\
\hline 6 & 5. 34 & 68.38 & 0.0775 & & \\
\hline 7 & 4. 82 & 61.65 & 0.0398 & & \\
\hline 8 & 4.00 & 51.26 & 0.0438 & & \\
\hline 9 & 4. 64 & 59.33 & 0.0794 & & \\
\hline 10 & 4. 68 & 59.93 & 0.0691 & & \\
\hline Mean & 4. 50 & 57.60 & 0.0630 & & \\
\hline Std. Error & 0.17 & 2. 22 & 0.0046 & & \\
\hline Combined & 4. 48 & 57.33 & 0.0269 & & \\
\hline
\end{tabular}

\section{Acid rinsed NPS}




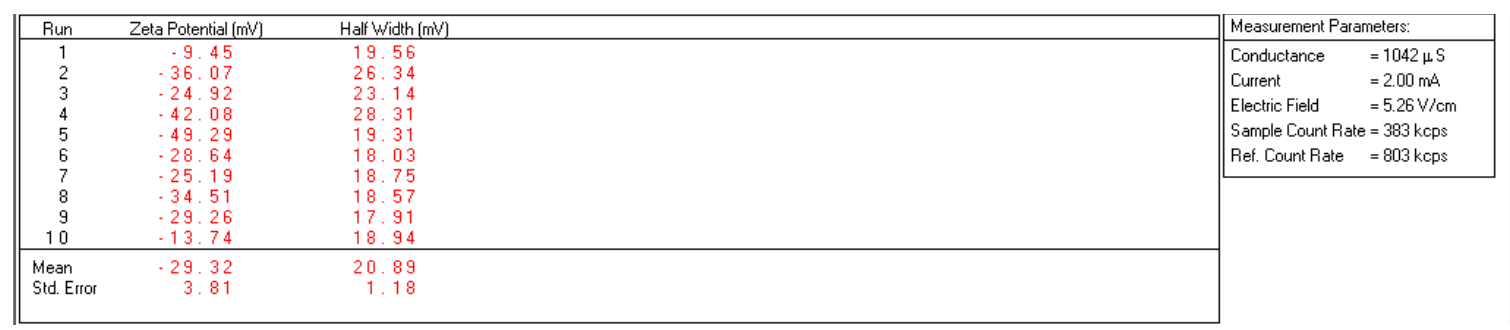

Zeta potential of $\mathrm{CS}_{2}$-modified NPs

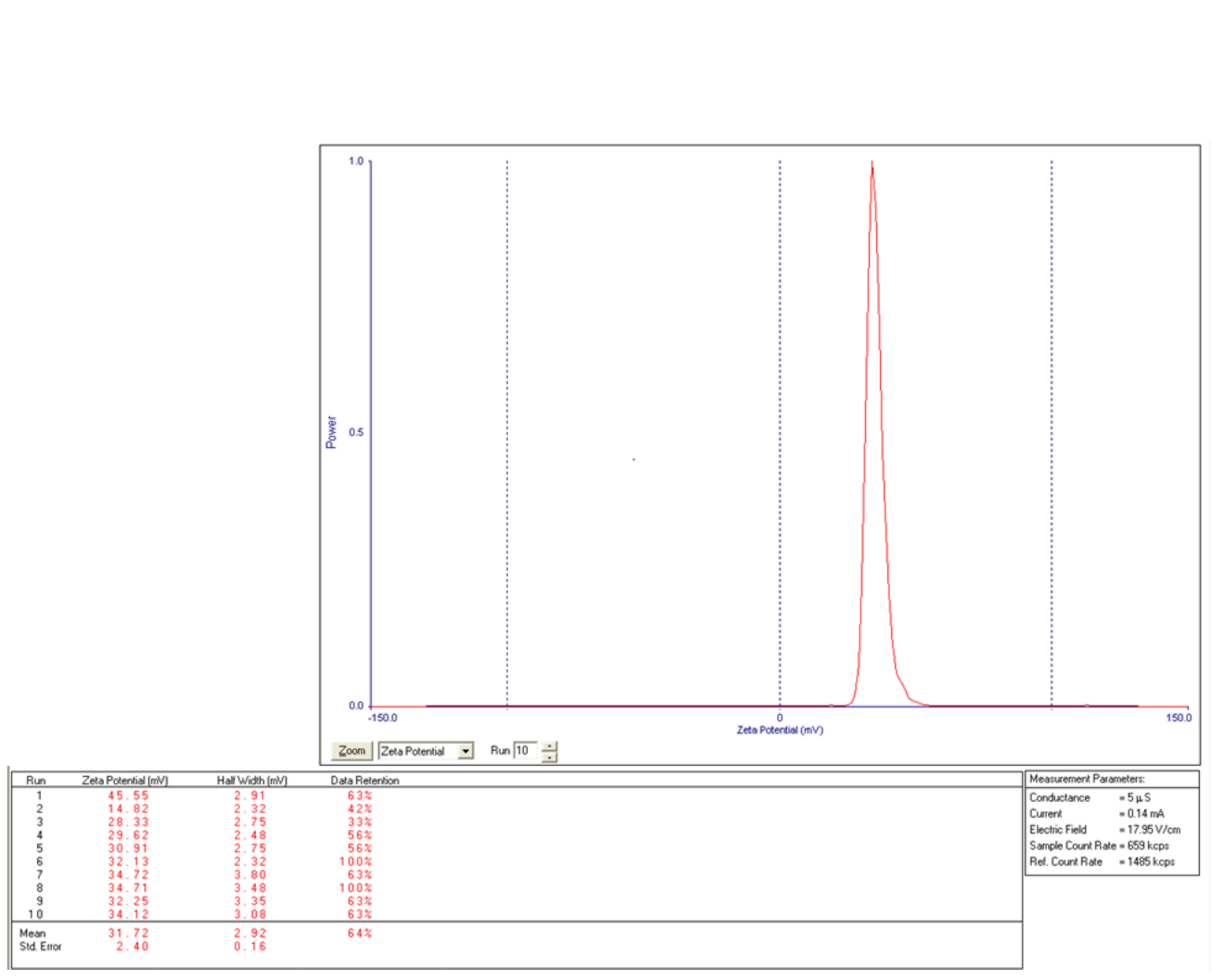

Zeta potential of CML with lipid 1 


\section{A.3. EXCITATION-EMISSION SPECTRA}
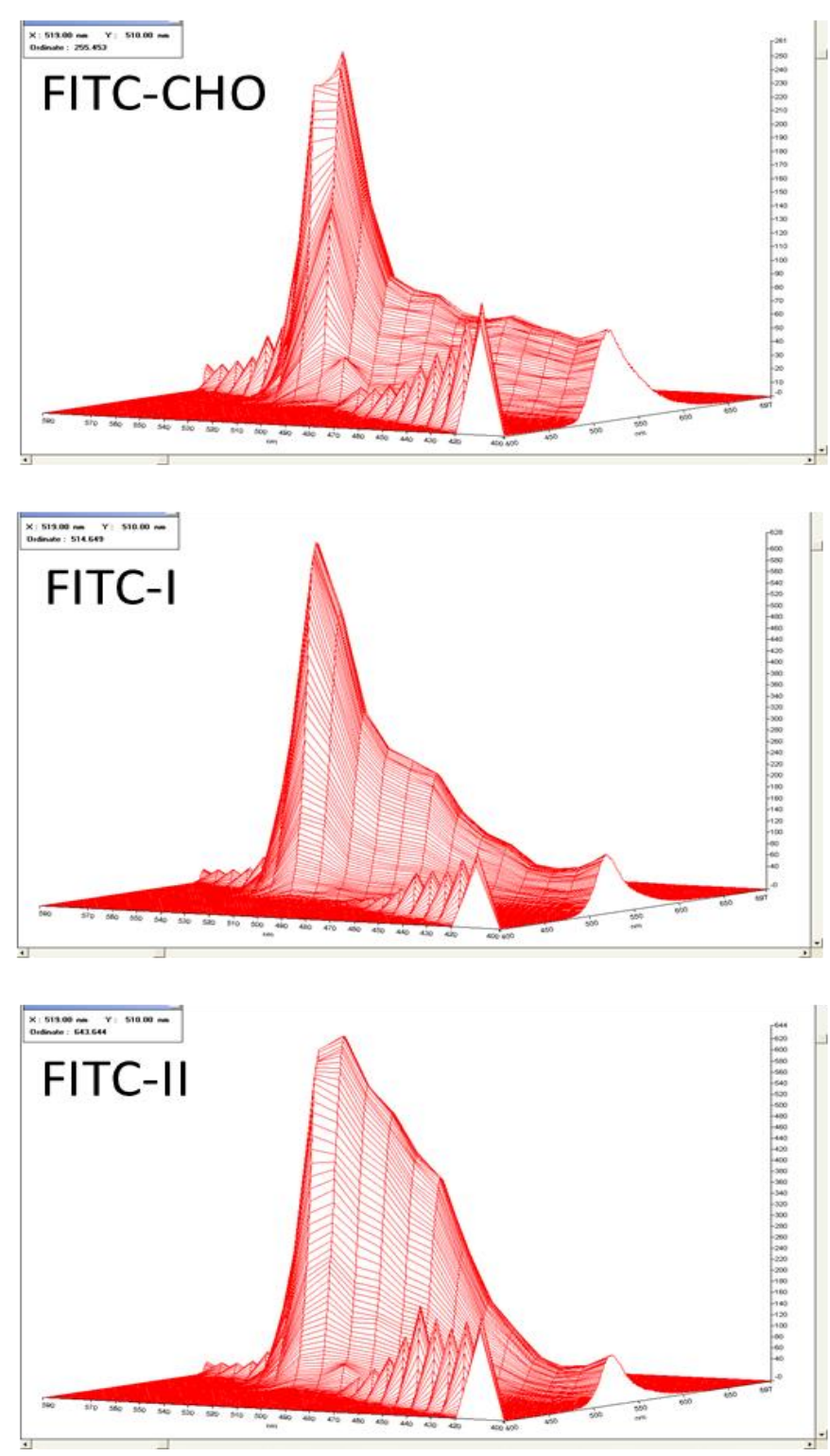

Excitation-emission spectra for FITC derivatives 


\section{A.4. DYNAMIC LIGHT SCATTERING MEASUREMENTS}

A

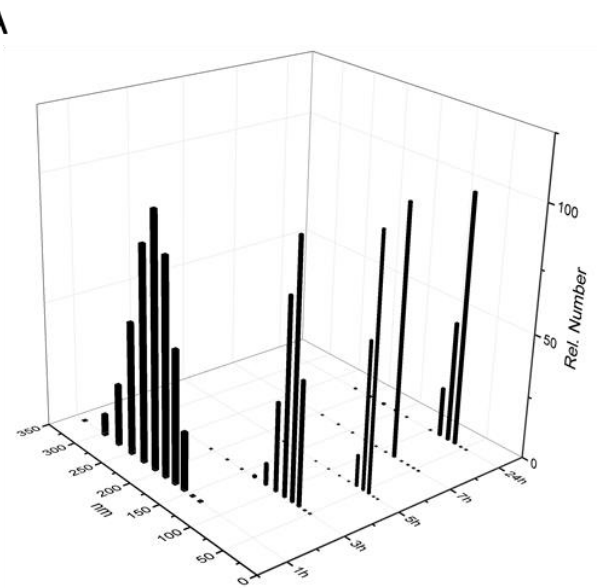

B

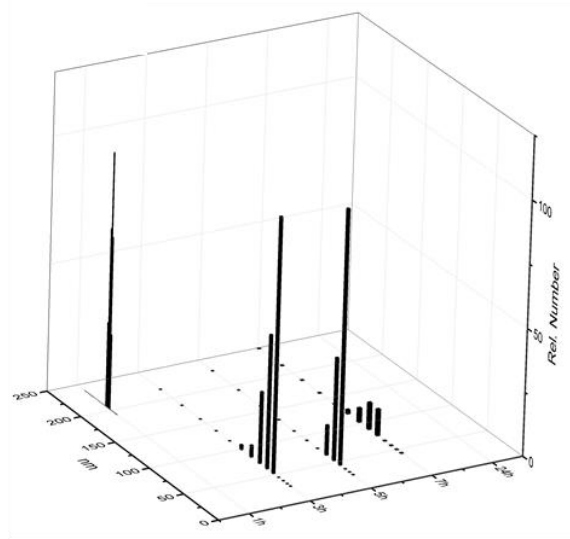

C

3D stack plots of DLS

measurements over 24 mixing time with lipid 1 (A), 2 (B), and $3(C)$.

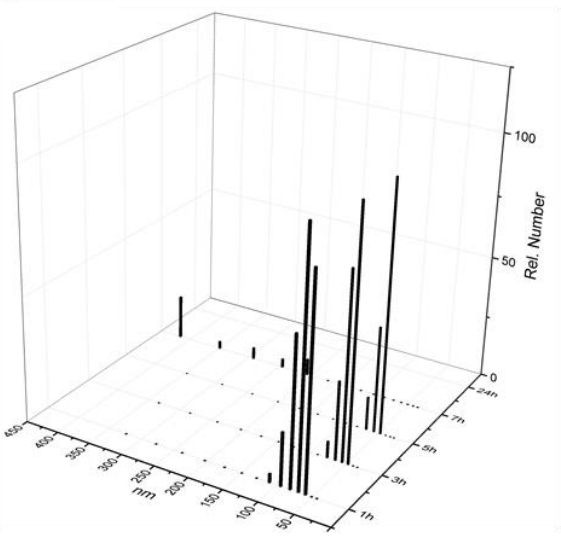




\section{A.5. FLUORESCENCE AND BRIGHT-FIELD MICROSCOPE IMAGES}

Fluorescence (green filter) and bright-field microscope images were acquired after

$2 \mathrm{~h}$ treatment with CML/SNP formulations on an EVOS FL Cell Imaging System at magnifications of $10 x$ or $20 x$. 

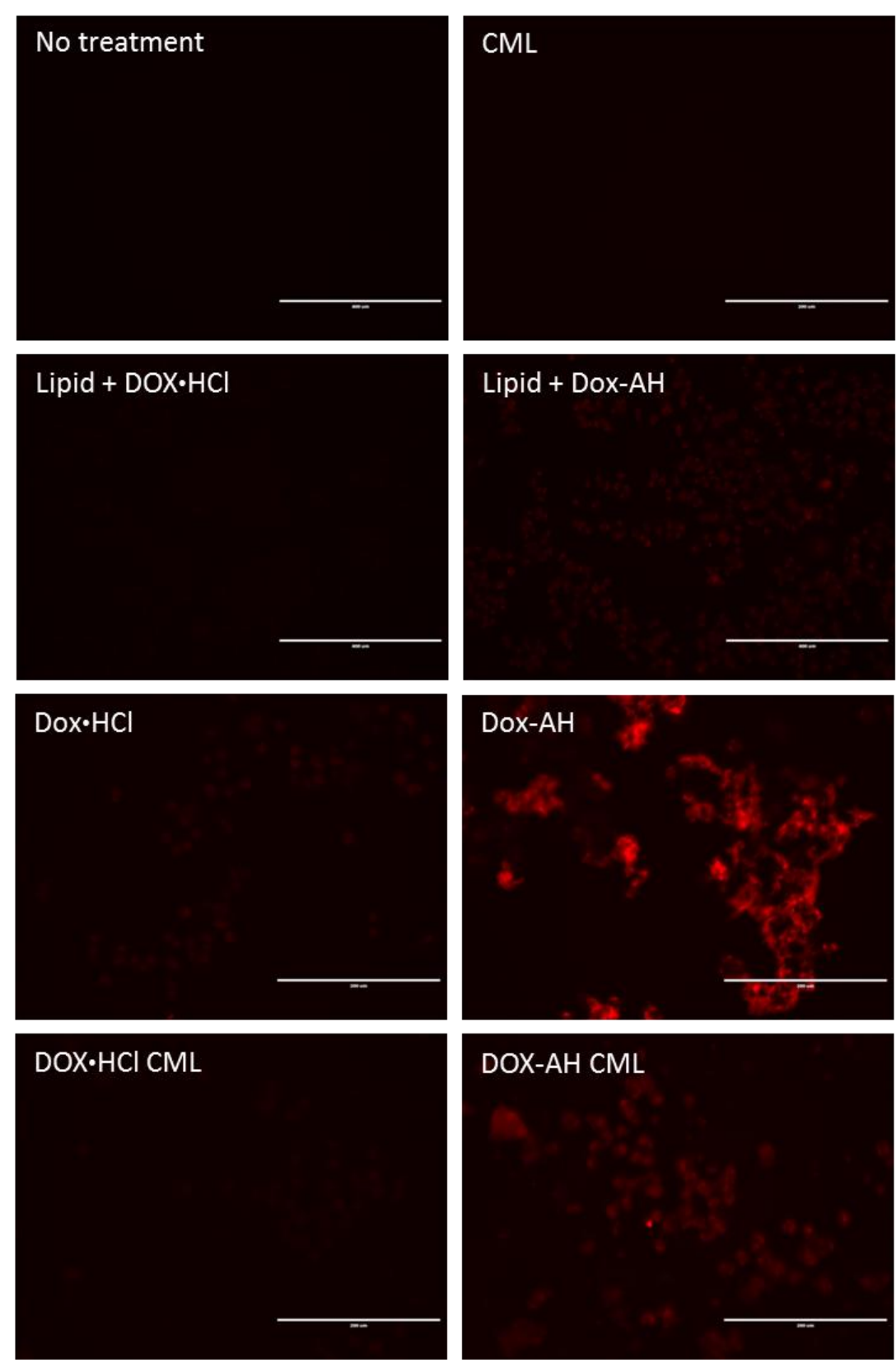

Fluorescence images of MCF-7 cells after $2 \mathrm{~h}$ treatment with SNP formulations and controls. 

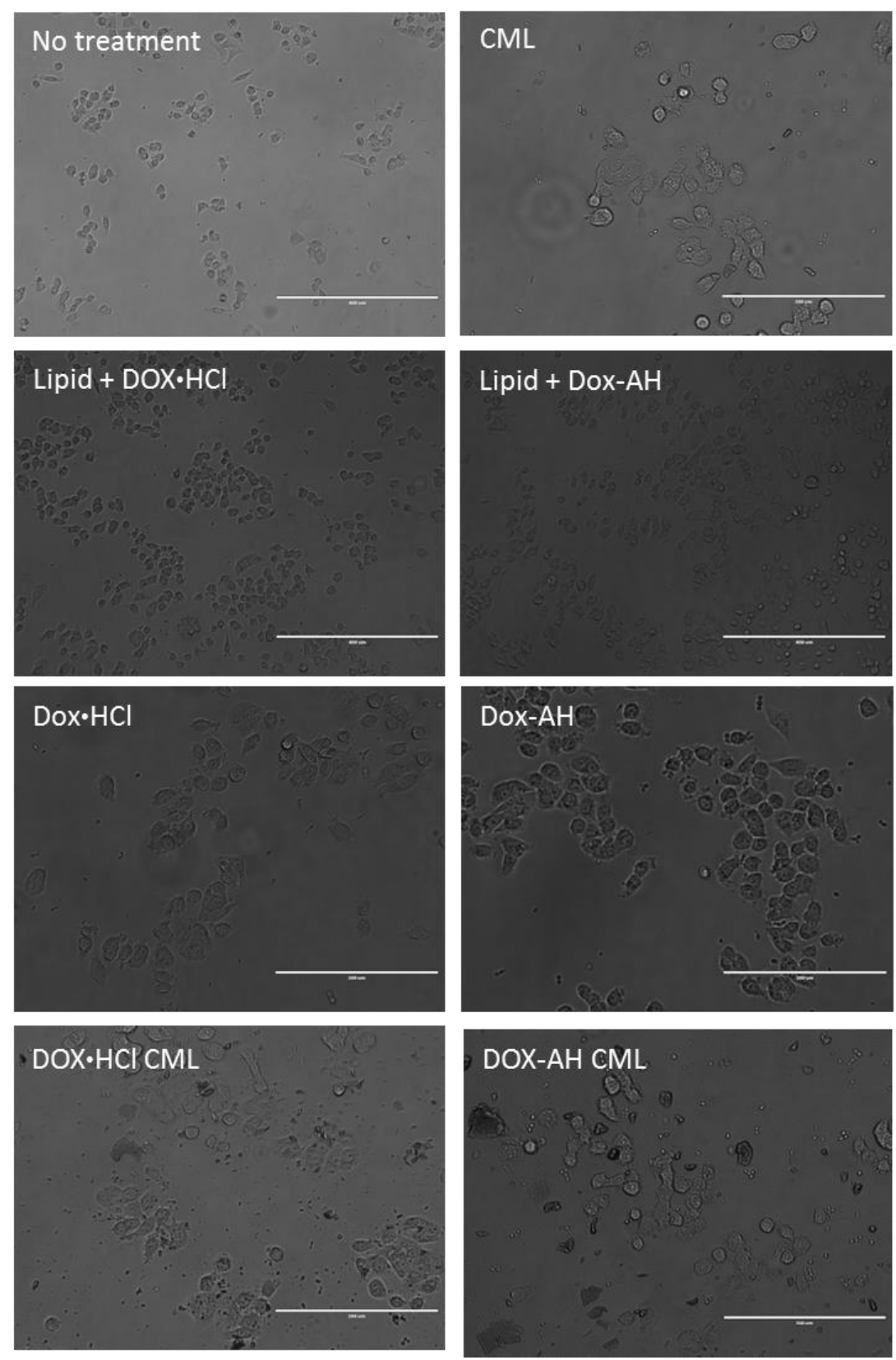

Bright-field images of MCF-7 cells after $2 \mathrm{~h}$ treatment with SNP formulations and controls. 


\section{A.6. COPYRIGHT PERMISSIONS}

Figure 1.6. Chem. Commun. (Cambridge, U. K.) 2011, 47, 5130-5141. Copyright (2011) Royal Society of Chemistry. License number 3505490358928.

Figure 1.9. Biomaterials 2011, 32 (34), 8999-9010. Copyright (2011) Elsevier. License number 3505631083750 .

Figure 1.10. Molecular pharmaceutics 2005, 2 (3), 194-205. Copyright (2005) American Chemical Society. Permission granted without license.

Figure 1.11. Biomaterials 2014, 35 (4), 1240-1248. Copyright (2014) Elsevier. License number 3506030114981.

Figure 1.12. J. Am. Chem. Soc. 2010, 132 (2), 552-557. Copyright (2010) American Chemical Society. Permission granted without license.

Figure 1.13. Biomaterials 2013, 34 (14), 3706-3715. Copyright (2013) Elsevier. License number 3506111061682.

Figure 1.14. ACS Nano 2012, 6 (5), 4157-4168. Copyright (2012) American Chemical Society. Permission granted without license.

Figure 3.22. Environ. Sci. Technol. 2007, 41 (18), 6484-6490. Copyright 2007 American Chemical Society. Permission granted without license. 
Chapter 2 reproduces work with kind permission from Springer Science and Business Media: Springer, Metabolomics 2012, 8, 989-996. Mattingly, S. J.; Xu, T.; Nantz, M. H.; Higashi, R. M.; Fan, T. W. -M. 


\section{A.7. LIST OF ABBREVIATIONS}

ATR attenuated total reflectance

CML cationic magnetoliposome

DLS dynamic light scattering

DMEM Dulbecco's modified eagle medium

DOPE 1,2-di-(9Z-octadecenoyl)-sn-glycero-3-phosphoethanolamine

Dox $\cdot \mathrm{HCl}$ Doxorubicin hydrochloride

Dox-AH Doxorubicin acyl hydrazone

DRIFT diffuse reflectance infrared Fourier transform

EA elemental analysis

EES excitation-emission spectrum

FITC fluorescein isothiocyanate

FT-ICR-MS Fourier-transform ion-cyclotron resonance mass spectrometry

MS mass spectrometry

NP iron oxide nanoparticle

PBS phosphate buffered saline

QAO quaternary aminooxy

QDA quaternary dodecyl aminooxy

*QDA ${ }^{13} \mathrm{CD}_{3}$-labeled QDA 
QOA quaternary octyl aminooxy

SNP silica-coated iron oxide nanoparticle

TGA thermogravimetric analysis 


\section{CURRICULUM VITAE}

\section{Stephanie Mattingly}

(502) 649-1948

sjmatti@gmail.com

529 Camden Ave.

Louisville, KY

40215

\section{Education}

Candidate for PhD in Chemistry, University of Louisville

M.S., Chemistry, University of Louisville

(Fall 2011 - present)

December 2011

B.A., Music with High Honors, University of Louisville

May 2005

B.A., Humanities with High Honors, University of Louisville

May 2005

\section{Honors and Awards}

The Graduate Dean's Citation

2014

Doctoral Dissertation Completion Award

2014

Overseers Scholar

2004-2005

Dean's List

2003-2006

Music School Scholarship

2001-2005

KEES Scholarship

2000-2004

Trustees Academic Scholarship

2001-2004

Hellman Music Scholarship

2002

B. Owen Memorial Scholarship

2001-2002

E. M. Aldrich Scholarship

2001

\section{Experience}

\section{Teaching Assistant}

University of Louisville (2009 - present)

Contemporary methods of synthesis and analysis (CHEM 528)

Separations and spectroscopy (CHEM 527)

Organic chemistry laboratory (CHEM 343, CHEM 344)

Organic chemistry recitation (CHEM 341)

General chemistry laboratory (CHEM 207, CHEM 208) 
Research

Organic synthesis

Mass spectral analysis

Cell culture (MCF-7 cell line)

Nanomaterial characterization

\section{Instrumental}

- ${ }^{1} \mathrm{H}$ and ${ }^{13} \mathrm{C}, 1 \mathrm{D}$ and 2D NMR

- Fourier transform-ion cyclotron resonance-mass spectrometry

- Column and thin-layer chromatography including flash chromatography

- UV-Vis and fluorescence spectroscopy

- Diffuse reflectance infrared Fourier transform spectroscopy
University of Louisville (Spring 2008 - present)

- Infrared spectroscopy and attenuated total reflectance IR

- Zeta potential and dynamic light scattering measurements

- Fluorescence microscopy

O High-performance liquid chromatography

- Thermogravimetric analysis

\section{Presentations}

Mattingly, S. J.; Hobbing, K. R.; Clark, G. J.; Nantz, M. H. Lipid-coated Nanoparticles for Drug Delivery. Poster 542; presented at the $66^{\text {th }}$ Southeastern Regional Meeting of the American Chemical Society, Nashville, TN; October 18, 2014.

Lorkiewicz, P. K.; Higashi, R. M.; Mattingly, S. J.; Nantz, M. H.; Moseley, H. N. B.; Lane, A. N.; Fan, T. W.-M. Chemoselective Capture of Carbonyl-Containing Metabolites for Stable Isotope Resolved Metabolomic Analysis of Crude Cell Extracts by FTICR-MS. Poster 365; presented at the $61^{\text {st }}$ ASMS Conference on Mass Spectrometry and Allied Topics, Minneapolis, MN; June 1, 2013.

Mattingly, S. J.; Biswas, S.; Peace, C. J.; Beglin, K. A.; Clark, G. J.; Nantz. M. H. Dual-Purpose Cancer Therapy Using Functionalized Iron Oxide Nanoparticles. Poster 86; presented at the International Conference on the Scientific and Clinical Applications of Magnetic Carriers, Minneapolis, MN; May 24, 2012.

Nantz, M. N., Mattingly, S. J., Laulhe S., Biswas, S. Oximation Applications for Analysis of Carbonyl Metabolites. Talk presented at the Sino-US Forum on Chemical Biology, 28th Chinese Chemical Society Congress, Chengdu, China, April 13, 2012.

Mattingly, S. J., Biswas, S., Peace, C. J., Beglin, K. A., Nantz, M. H. Dual-purpose Cancer Therapy Using Functionalized Iron Oxide Nanoparticles. Talk presented at the Kentucky Nanotechnology Symposium 2012, Bowling Green, KY; March 30, 2012. 
Mattingly, S. J.; Xu, T.; Higashi, R. M.; Fan, T. W. -M.; Nantz, M. H. Profiling Oxidized Cellular Metabolites: A Carbonyl Capture Approach. Poster 432; presented at the $63^{\text {rd }}$ Southeastern Regional Meeting of the American Chemical Society, Richmond, VA; October 28, 2011. And poster 5 ; presented at the 2012 Symposium of the Institute for Molecular Diversity and Drug Design, Louisville, KY; March 13, 2012.

Biswas S.; Gordon, L.; Deb, B.; Mattingly, S. J.; Massey, A. P.; Clark, G. J.; Nantz, M. H. Magnetic Lipid Particles (MLPS): A New Class of Iron Oxide Nanoparticles for Gene Transfer. Poster 10; presented at the Inaugural Conference of the American Society for Nanomedicine, Potomac, MD; October 23, 2009.

Nantz, M. H.; Biswas S.; Gordon, L.; Deb, B.; Mattingly, S. J.; Massey, A. P.; Clark, G. J. Magnetic Lipid Particles (MLPS): Iron Oxide Nanoparticles for Gene Transfer. Talk presented at the Symposium on Chemical Applications of Nanomaterials at the Federation of Analytical Chemistry and Spectroscopy Studies 2009 Conference, Louisville, KY; October 19, 2009.

Biswas S.; Gordon, L.; Deb, B.; Mattingly, S. J.; Massey, A. P.; Clark, G. J.; Nantz, M. H. Magnetic Lipid Particles (MLPs): A New Class of Iron Oxide Nanoparticles for Gene Transfer. Poster 18; presented at the $11^{\text {th }}$ Annual IMD ${ }^{3}$ Symposium, Louisville, KY; March 10, 2009.

\section{Publications}

Mattingly, S. J.; Clark, G. J.; Nantz, M. H., Cationic Magnetoliposomes for Drug Delivery. Submitted to Journal of Controlled Release, December, 2014.

Gupta, K.; Mattingly, S. J.; Knipp, R. J.; Afonin, K. A.; Viard, M.; Bergman, J. T.; Stepler, M.; Blumenthal, R.; Nantz, M. H.; Puri, A.; Shapiro, B. A. Oxime-ether lipids containing hydroxylated head groups are superior siRNA delivery agents than their non-hydroxylated counterparts. Submitted to Langmuir, December, 2014.

Knipp, R. J.; Mattingly, S. J.; Nantz, M. H., Magnetic-field induced hydrolysis as a mechanism for rapid release of nanoparticle-bound substrates. Submitted to Advanced Materials Interfaces, October, 2014.

Mattingly, S. J.; Xu, T.; Nantz, M. H.; Higashi, R. M.; Fan, T. W. -M., A carbonyl capture approach for profiling oxidized metabolites in cell extracts. Metabolomics 2012, 8. 989996. 


\section{$\underline{\text { References }}$}

Dr. Michael Nantz

2210 S. Brook St.

Shumaker Research Bldg. rm. 345

University of Louisville

Louisville, KY 40208

(502) 852-8069

michael.nantz@louisville.edu

Dr. Teresa Fan

789 S. Limestone

523 BioPharm Complex

University of Kentucky

Lexington, KY 40536

(859) 218-1028

twmfan@gmail.com
Dr. Christopher Burns

2320 S. Brook St.

Department of Chemistry, rm. 335

University of Louisville

Louisville, KY 40292

(502) 852-5977

christopher.burns@louisville.edu

Dr. Pawel Lorkiewicz

Diabetes and Obesity Center

580 S. Brook St.

Baxter II, Rm 421C

University of Louisville

Louisville, KY 40202

(502) 852-5750

pawell78@gmail.com 